\title{
Ultraschallabsorptionsspektroskopie zur Untersuchung schneller molekularer Prozesse in Alkylglykosid-Lösungen
}

\author{
Dissertation \\ zur Erlangung des Doktorgrades der Mathematisch-Naturwissenschaftlichen Fakultäten \\ der Georg-August-Universität zu Göttingen
}

vorgelegt von

Dipl.-Phys. Julian Haller

aus Göttingen

Göttingen 2008 
D7

Referent:

Prof. Dr. W. Lauterborn

Korreferent

PD Dr. C. Jooß

Tag der mündlichen Prüfung: 17.06 .2008 
Für meine Familien 

I've met some people along the way, Some of them split some of them stay, Some of them walk some walk on by, I've got a few friends I'll love till I die From all of these people I try to learn, Some of them shine some of them burn, Some of them rise some of them fall, For good or bad I've known them all.

The Bouncing Souls - True Believers 



\section{INHALTSVERZEICHNIS}

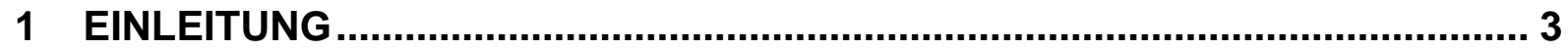

2 ULTRASCHALLABSORPTIONSSPEKTROSKOPIE ...................................... 5

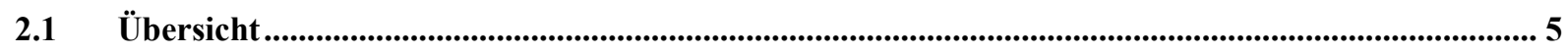

2.2 Die klassische Dämpfung .................................................................................................................... 7

2.3 Molekulare Prozesse in Flüssigkeiten .......................................................................................................... 8

2.4 Das Ultraschalltransmissionsverfahren.................................................................................................... 15

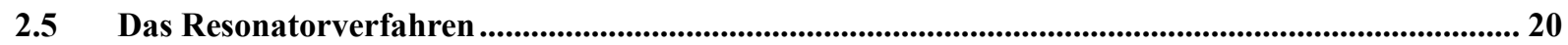

3 ALKYLGLYKOSIDE............................................................................. 27

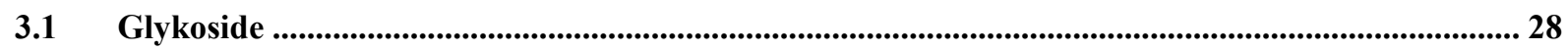

K.2 Konformationsänderungen von Glykosiden ....................................................................................... 30

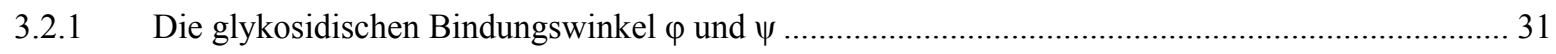

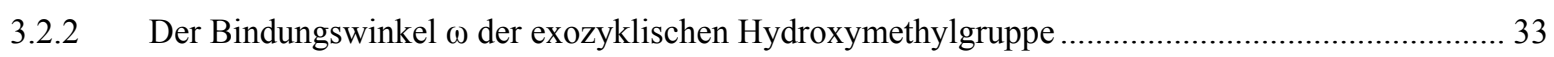

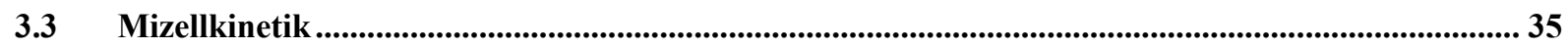

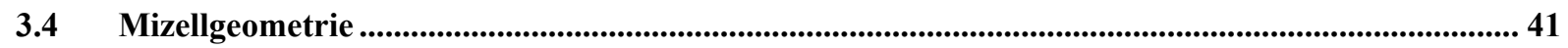

3.5 Mizellkinetik und -geometrie von Alkylglykosiden ....................................................................44

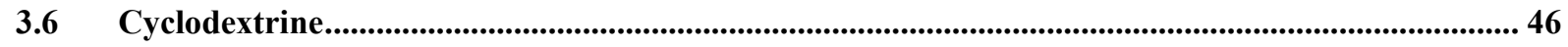

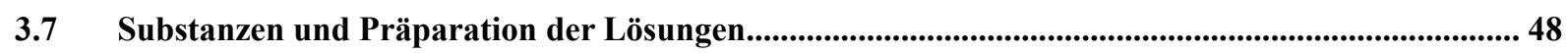

4 ERGEBNISSE UND AUSWERTUNG DER SPEKTREN ............................... 51

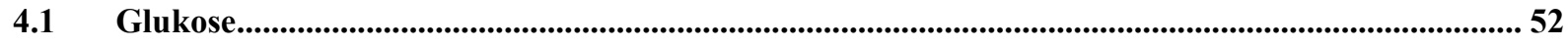

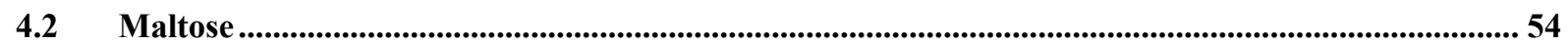

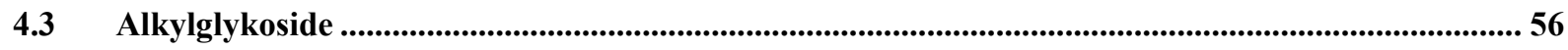

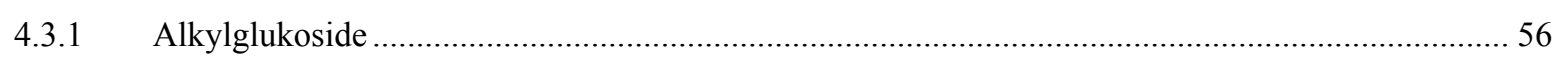




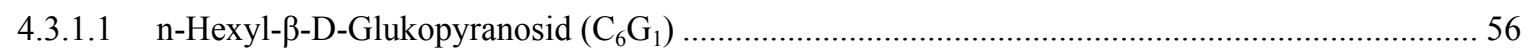

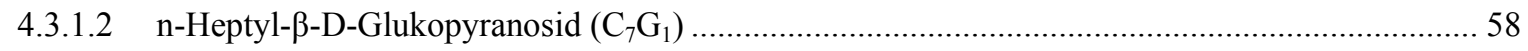

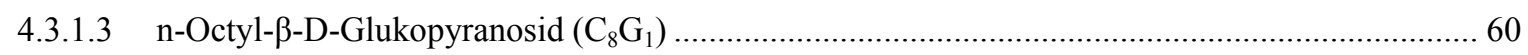

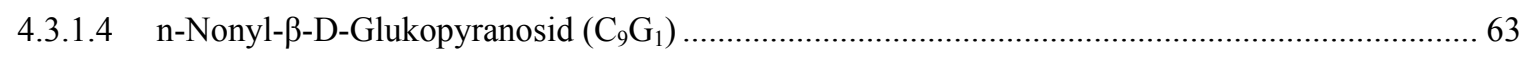

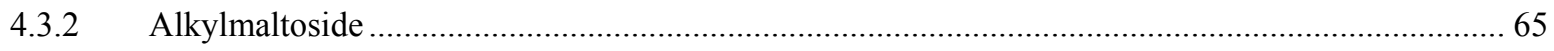

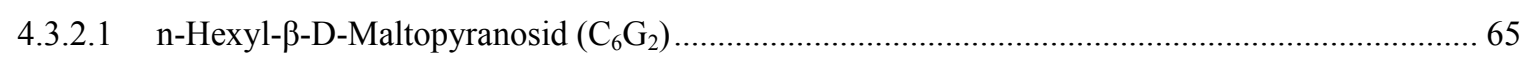

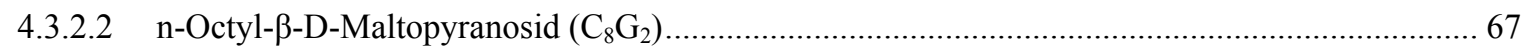

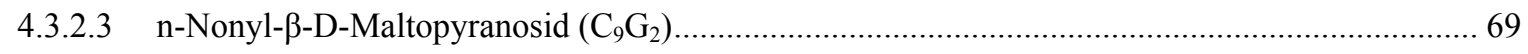

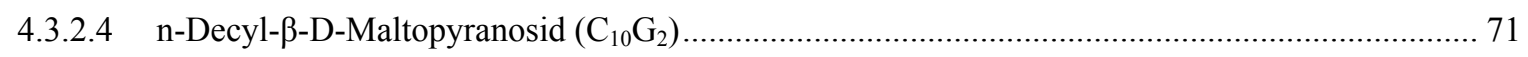

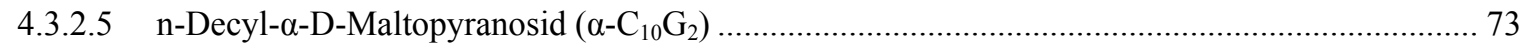

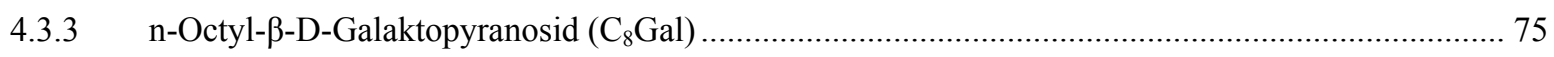

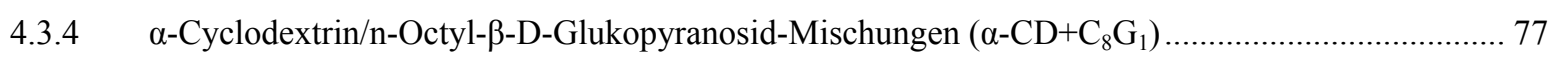

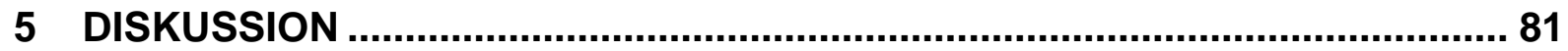

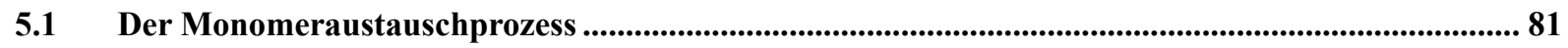

5.2 Die Rotation um die glykosidischen Bindungswinkel ( $\varphi$-, $\psi$-Rotation) .......................................... 93

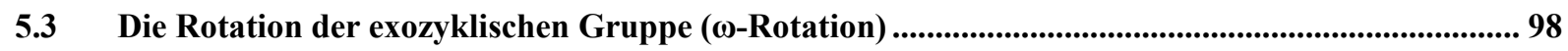

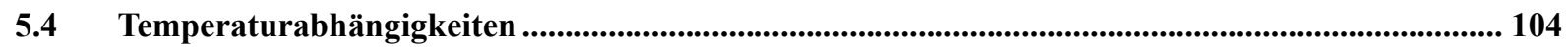

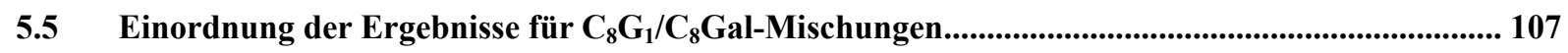

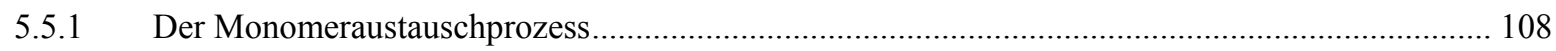

5.5.2 Rotation der glykosidischen Bindung und der exozyklischen Gruppe........................................ 109

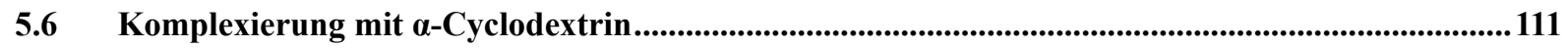

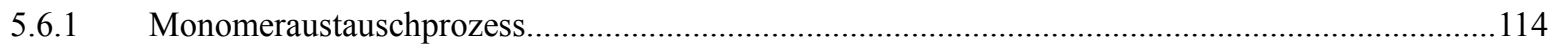

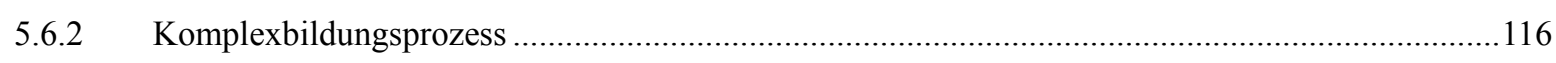

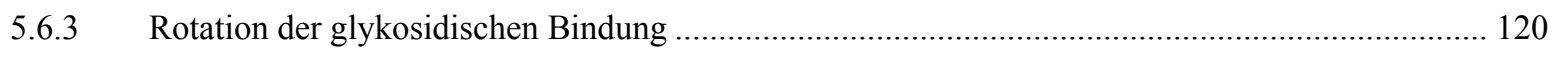

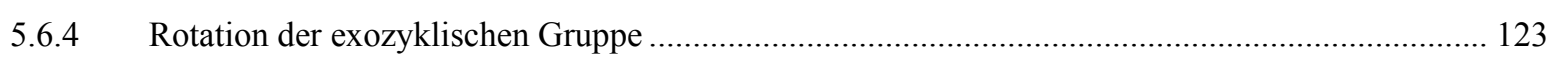

6 ZUSAMMENFASSUNG UND AUSBLICK...................................................125

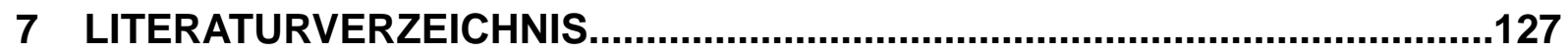

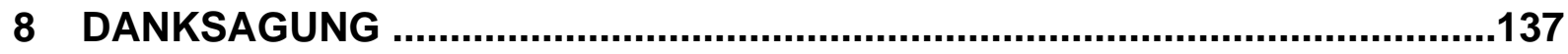




\section{$1 \quad$ Einleitung}

Alkylglykoside sind Moleküle, die aus einem Glykosid (einem Zuckerteil) und einer Alkylkette (einer Kohlenwasserstoffkette) bestehen. Da Glykoside hydrophil (von gr. hydros - Wasser, philos - liebend, „wasserliebend“) sind, die Alkylteile wegen ihrer apolaren Struktur hingegen hydrophob (von gr. phobos - fürchtend, ,wasserfürchtend“), zählen Alkylglykoside zu den sogenannten Amphiphilen (von gr. amphis - beides, „beides liebend“). Amphiphile können aufgrund ihrer ambivalenten Struktur je nach Umgebung, Konzentration, Temperatur, molekularer Struktur und anderer (äußerer) Parameter in einer Lösung als Monomere vorliegen oder als Aggregate, wie zum Beispiel als flächenhafte Membranen oder als globuläre Mizellen (von nlat.: micella, Diminutiv zu lat.: mīca ,Krümchen')[1]. Die hier untersuchten Alkylglykoside aggregieren alle oberhalb einer bestimmten, systemabhängigen Konzentration, der $c m c$ (von engl. critical micelle concentration - kritische Mizellkonzentration), zu Mizellen.

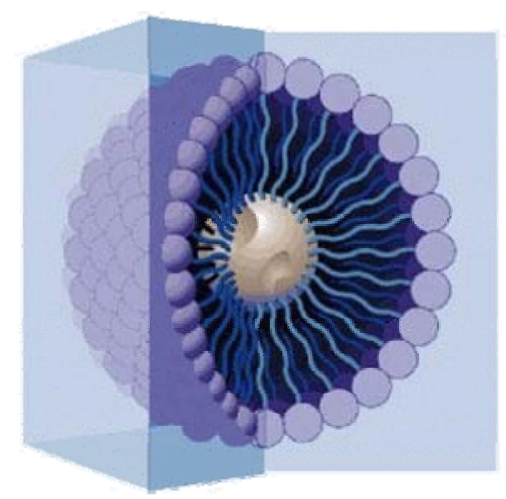

Abbildung 1: Schematische Darstellung einer Mizelle: Die einzelnen Monomere sind so angeordnet, dass die hydrophoben Teile durch die hydrophilen Teile (hier als Kugeln angedeutet) vom umgebenden Wasser abgeschirmt werden. Dadurch können im Inneren hydrophobe Bestandteile (hier beige dargestellt) in Wasser solubilisiert werden. Abbildung entnommen aus [2].

Diese Mizellen, bei denen die Moleküle so angeordnet sind, dass die hydrophoben Molekülteile in einer Kugelform durch die hydrophilen Molekülteile vom umgebenden Wasser abgeschirmt werden, ermöglichen es, in ihrem Inneren hydrophobe Bestandteile (also z.B. Fette und Öle) zu solubilisieren. Dieser Vorgang findet bei nahezu allen Wasch-, Putz- und Reinigungsmitteln Anwendung [3], aber auch im Körper (zum Beispiel ermöglichen Gallensäuremizellen die Verdauung der hydrophoben Vitamine A, D, E und K [4]) und in der Wissenschaft (zum Beispiel werden Membranproteine durch Mizellen aus Membranen ausgewaschen, um sie in wässriger Lösung untersuchen zu können [5]). Weiterhin kann mittels Mizellen ein zielgerichteter Medikamententransport im Körper stattfinden, indem die Wirkstoffe in Mizellen solubilisiert durch den Körper transportiert werden, bevor sie an den gewünschten Stellen durch dort vorherrschende physiko-chemische Bedingungen (z.B. den pHWert oder die Salzkonzentration) freigesetzt werden [6], [7].

In der vorliegenden Arbeit wird mithilfe der Ultraschallabsorptionsspektroskopie der sogenannte Monomeraustauschprozess [8]-[14] (siehe Abbildung 2) zwischen Alkylglykosid-Mizellen und der Lösungsmittelphase untersucht. Aus den Ergebnissen lassen sich Informationen über die Geschwindigkeitskonstanten und damit Gleichgewichtskonstanten sowie das (isentropische) Reaktionsvolumen dieses Prozesses gewinnen. Das Verhalten 
dieser Parameter kann dann in Abhängigkeit von den verschiedenen Glykosid-Gruppen oder der Alkylkettenlänge betrachtet werden, um deren Einfluss auf den Monomeraustausch kennenzulernen.

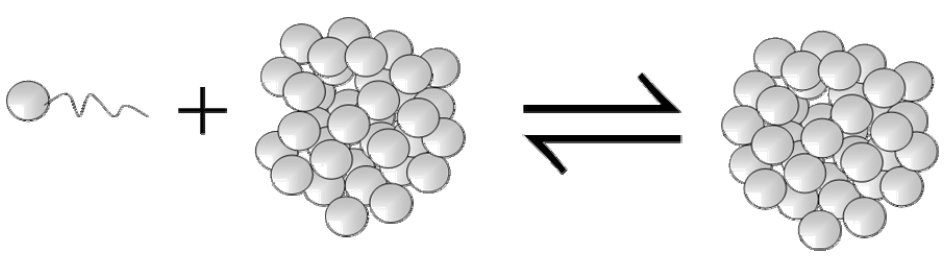

Abbildung 2: Schematische Darstellung des Monomeraustauschprozesses in mizellaren Systemen:

Ein Monomer und ein Aggregat aus $n$ Molekülen werden zu einem Aggregat aus $n+1$ Molekülen.

Das spezielle Interesse an Alkylglykosiden beruht zum einen auf der Tatsache, dass diese Substanzklasse wegen ihrer positiven Eigenschaften wie biologischer Abbaubarkeit, Hautfreundlichkeit, einfacher Gewinnung und Umweltverträglichkeit für die industrielle Fertigung von Wasch- und Reinigungsmitteln zunehmend an Bedeutung gewinnt [15]. Zum anderen darauf beruht es darauf, dass ihre hydrophile Kopfgruppe, also der Zuckerrest, eine extrem wichtige Rolle in biologischen Vorgängen spielt.

Ein weiteres besonderes Augenmerk wird daher in der vorliegenden Arbeit auf molekulare Konformationsänderungen der Zuckerreste gelegt, da diese mit den wohlbekannten von Lösungen reiner Zucker verglichen werden können. Eine interessante Fragestellung betrifft die mögliche Beeinflussung dieser Konformationsänderungen durch die Anwesenheit einer Alkylkette oder das Vorliegen von direkten Nachbarn in einer Mizelle. Solche Untersuchungen sind von großer Relevanz für das grundlegende Verständnis von sogenannten ,induced-fit'Komplexbildungen [16] und der Frage, ob möglicherweise Alkylglykosid-Mizellen vom Körper in bestimmten Fällen als Zuckerkomplexe erkannt werden könnten, um so z.B. gewünschte Stoffe wie Medikamente in den Mizellen solubilisiert an spezifischen Stellen in den Körper einzubringen.
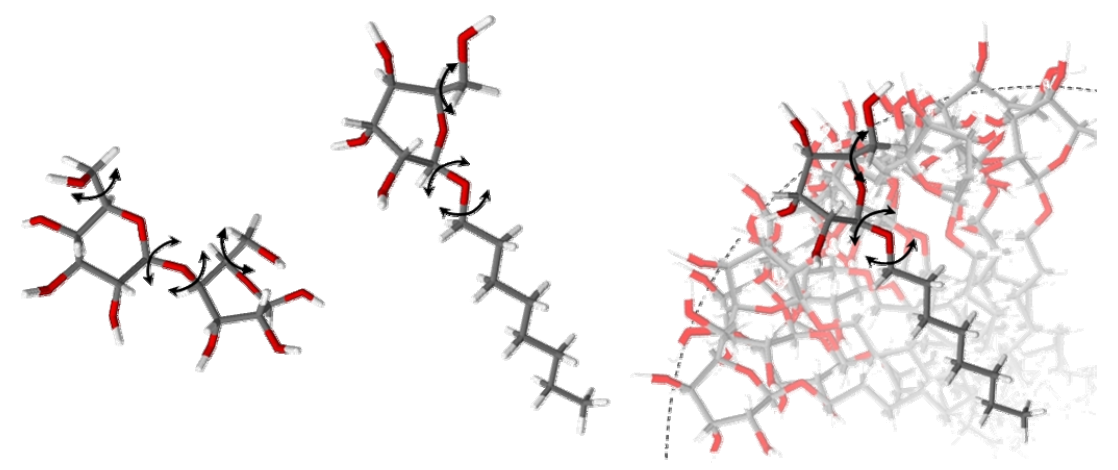

\section{Abbildung 3: Schematische Darstellung zu möglichen Konformationsänderungen des Maltose- Moleküls (links), des Alkylglykosid-Monomers $\mathrm{C}_{8} \mathrm{G}_{1}$ (mitte) und des gleichen Moleküls in einer Mi- zelle (rechts).}

In der vorliegenden Arbeit werden molekulare Prozesse mithilfe der Ultraschallabsorptionsspektroskopie [17] in einem Frequenzbereich von ca. $100 \mathrm{kHz}$ bis ca. $400 \mathrm{MHz}$ untersucht. Damit können Relaxationszeiten von etwa $0.5 \mathrm{~ns}$ bis $1.5 \mu$ s ermittelt werden. 


\section{Ultraschallabsorptionsspektroskopie}

\section{1 Übersicht}

Eine harmonische Schallwelle lässt sich an einem festen Ort als zeitliche Druckschwankung

$$
\begin{aligned}
p(t) & =p_{0} \cdot \sin \left(\omega \cdot t+\Phi_{0}\right) \\
& =p_{0} \cdot \sin \left(2 \cdot \pi \cdot f \cdot t+\Phi_{0}\right)
\end{aligned}
$$

beschreiben.

Dabei ist $p_{0}$ die Schalldruckamplitude, $f$ und $\omega$ sind die Frequenz bzw. Kreisfrequenz der Schallwelle und $\Phi_{0}$ ist ein Phasenwinkel. Eine solche Schallwelle erfährt in jedem Medium längs eines Weges $x$ eine exponentielle Dämpfung mit einem Dämpfungsexponenten $\alpha$ :

$$
p_{0}(x)=p_{0}(0) \cdot \exp \{-\alpha(f) \cdot x\}
$$

In der Ultraschallabsorptionsspektroskopie erlaubt die Messung dieses frequenzabhängigen Dämpfungsexponenten $\alpha$ einen Einblick in die molekularen Prozesse, die in einer Flüssigkeit ablaufen. Der Zusammenhang zwischen der Dämpfung in einer Flüssigkeit und den zugrundeliegenden molekularen Prozessen sowie die Möglichkeit, konkrete Relaxationszeiten und Reaktionsvolumina der molekularen Prozesse aus den Ultraschallabsorptionsspektren zu gewinnen, werden in Kapitel 2.3 behandelt.

In der vorliegenden Arbeit wurde die Dämpfung von Ultraschallwellen mit Frequenzen von etwa $100 \mathrm{kHz}$ bis $400 \mathrm{MHz}$ bestimmt. Dazu wurde im Allgemeinen die Flüssigkeit zwischen zwei piezoelektrische Kristalle gebracht, von denen einer als Sendewandler (,Lautsprecher') und einer als Empfangswandler (,Mikrofon') diente. Piezoelektrische Kristalle wandeln aufgrund des sogenannten inversen piezoelektrischen Effektes [18] eine elektrische Spannung in eine Ausdehnung bzw. umgekehrt aufgrund des piezoelektrischen Effektes eine Längenänderung in eine elektrische Spannung um. Die spezielle kristallografische Anordnung der Atome in diesen Substanzen reagiert auf ein elektrisches Feld mit einer leichten Verschiebung der Atome. Da dieser Effekt nahezu instantan abläuft, kann durch Anlegen einer Wechselspannung mit sehr hoher Frequenz eine Dickenschwingung des Kristalls und damit eine Ultraschallwelle mit gleicher Frequenz erzeugt werden.

Das zunächst relativ einfach klingende Prinzip der Ultraschallabsorptionsspektroskopie bedarf zweier unterschiedlicher Verfahren, des Ultraschalltransmissionsverfahrens und des Resonatorverfahrens mit einer jeweils relativ komplexen apparativen Umsetzung, die in Kapitel 2.4 beziehungsweise 2.5 beschrieben werden.

Ein großer Vorteil der Ultraschallabsorptionsspektroskopie gegenüber anderen Verfahren zur Bestimmung der molekularen Dynamik von Flüssigkeiten ist, dass die Dynamik der Flüssigkeit selbst durch die Messung nur extrem wenig beeinflusst wird, während bei vielen anderen Verfahren spezielle Marker, z.B. fluoreszierende Moleküle oder radioaktive Isotope, künstlich in die Flüssigkeit eingebracht werden müssen, die möglicherweise 
die untersuchte Dynamik beeinflussen. Ein weiterer Vorteil ist die allgemeine Verwendbarkeit, da praktisch alle molekularen Prozesse in Flüssigkeiten mit Volumenänderungen verbunden sind.

Nachteile hingegen sind zum einen die Tatsache, dass die Ultraschallabsorptionsspektroskopie unspezifisch ist, dass also detektierte molekulare Prozesse erst durch den Vergleich mehrerer Messungen einer konkreten Ursache zugeordnet werden können, und zum anderen das Vorliegen einer im folgenden Kapitel beschriebenen klassischen Hintergrunds-Dämpfung, die in jeder Flüssigkeit auftritt und sowohl die Messung als auch die Analyse in bestimmten Fällen erschwert oder sogar unmöglich macht. 


\subsection{Die klassische Dämpfung}

Die Dämpfung einer Schallwelle in einem Medium hat nach theoretischen Überlegungen [19] zunächst einmal zwei Ursachen: Zum einen führen die Druckschwankungen in dem betrachteten Medium dazu, dass Moleküle aneinander reiben und so Energie dissipiert. Dieser Anteil an der Schalldämpfung, die viskose Dämpfung $\boldsymbol{\alpha}_{\boldsymbol{v} \text { isk, }}$, lässt sich folgendermaßen berechnen:

$$
\alpha_{v i s k}=\frac{2 \cdot \pi^{2}}{c_{S}^{3} \cdot \rho} \cdot\left(\frac{4}{3} \cdot \eta_{S}+\eta_{V}\right) \cdot f^{2}
$$

Hierbei ist $c_{S}$ die Schallgeschwindigkeit, $\rho$ die Dichte des Mediums, $\eta_{S}$ die Scherviskosität und $\eta_{V}$ die Volumenviskosität. Zum anderen findet zwischen den (minimal erwärmten) Bereichen mit Überdruck und den (minimal kälteren) Bereichen mit Unterdruck permanent ein Wärmeaustausch statt, wobei wiederum Energie dissipiert. Diese thermische Dämpfung $\alpha_{\text {therm }}$ ist gegeben durch

$$
\alpha_{\text {therm }}=\frac{2 \cdot \pi^{2}}{c_{S}^{3} \cdot \rho} \cdot \frac{\Lambda}{C_{p}}\left(\frac{C_{p}}{C_{V}}-1\right) \cdot f^{2}
$$

mit der Wärmeleitzahl $\Lambda$, der spezifischen Wärmekapazität bei konstantem Druck $C_{p}$, bzw. bei konstantem Volumen $C_{V}$ und den anderen Größen wie in Gl. (2.3). Diese beiden, sogenannten klassischen Anteile der Dämpfung addieren sich zu

$$
\alpha_{\text {klass }}=\alpha_{\text {visk }}+\alpha_{\text {therm }}=\frac{2 \cdot \pi^{2}}{c_{S}^{3} \cdot \rho} \cdot\left[\left(\frac{4}{3} \cdot \eta_{S}+\eta_{V}\right)+\frac{\Lambda}{C_{p}}\left(\frac{C_{p}}{C_{V}}-1\right)\right] \cdot f^{2}
$$

so dass also der klassische Anteil der Dämpfung nach der Theorie proportional zu $f^{2}$ ist. Praktischerweise definiert man daher den Hintergrundsanteil der Dämpfung als (theoretischen) $B$-Wert:

$$
B_{\text {theo }} \equiv \frac{2 \cdot \pi^{2}}{c_{S}^{2} \cdot \rho} \cdot\left[\left(\frac{4}{3} \cdot \eta_{S}+\eta_{V}\right)+\frac{\Lambda}{C_{p}}\left(\frac{C_{p}}{C_{V}}-1\right)\right]
$$

Streng genommen können auch die Wärmekapazitäten, die Scherviskosität und die Schallgeschwindigkeit eine Frequenzabhängigkeit aufweisen - die entsprechenden hier als frequenzunabhängig betrachteten Größen sind daher als Messwert für $f \rightarrow \infty$ zu verstehen. Aufgrund der Proportionalität von $\alpha$ zu $f^{2}$ ist es in der Ultraschallabsorptionsspektroskopie üblich, die frequenznormierte Darstellung $\left(\alpha / f^{2}\right)$ zu betrachten, da diese für den klassischen Anteil einen frequenzunabhängigen Wert liefern sollte. Eine weitere übliche Darstellung betrachtet die Dämpfung entlang einer Wellenlänge, $(\alpha \lambda)$. Für diese beiden gebräuchlichen Größen ergibt sich der klassische Anteil zu

$$
\begin{aligned}
& \left(\frac{\alpha}{f^{2}}\right)_{\text {klass }}=\frac{B_{\text {theo }}}{c_{S}} \\
& (\alpha \lambda)_{\text {klass }}=B_{\text {theo }} \cdot f .
\end{aligned}
$$




\subsection{Molekulare Prozesse in Flüssigkeiten}

$\mathrm{Zu}$ dem beschriebenen klassischen Anteil der Dämpfung kommen nun noch Anteile dazu, die auf den in dieser Arbeit interessierenden molekularen Vorgängen beruhen. Zum Verständnis des Einflusses solcher molekularer Vorgänge auf die Ultraschalldämpfung sind zunächst einige grundlegende Betrachtungen über molekulare Prozesse notwendig:

Die Moleküle in einer Flüssigkeit unterliegen der Brownschen Molekularbewegung; zudem sind auch Moleküle selbst nicht vollkommen starr, sondern haben teilweise innere Konformations-Freiheitsgrade, so dass durch die thermischen Stöße der Nachbarmoleküle Konformationsänderungen auftreten können. Betrachtet man allgemein ein System, das in zwei möglichen Zuständen vorliegen kann, so kann man für dieses System eine Abhängigkeit des Potentials von einem Reaktionsparameter, z.B. dem Volumen des Systems, finden. In Abbildung 4 ist eine solche Abhängigkeit schematisch dargestellt. Das System kann den Zustand A oder den Zustand B einnehmen, der dem Volumen $V_{\mathrm{A}}$ bzw. $V_{\mathrm{B}}$ entspricht. In einer Flüssigkeit im Gleichgewicht stehen die Anzahlen von Systemen im Zustand A, $c(\mathrm{~A})$, und Systemen im Zustand B, $c(\mathrm{~B})$, in einem festen Verhältnis zueinander, das durch die Boltzmann-Verteilung gegeben ist. Im gegebenen Fall ist $c(\mathrm{~B})>c(\mathrm{~A})$, da Zustand $\mathrm{B}$ energetisch um $\Delta H$ günstiger als Zustand A ist. Jedes System kann durch thermische Stöße von Zustand A in Zustand B übergehen und umgekehrt - allerdings nur, wenn die durch den Stoß übertragene Energie groß genug ist, um den Potentialwall $\Delta \mathrm{H}_{\mathrm{A}}{ }^{\#}$ bzw. $\Delta \mathrm{H}_{\mathrm{B}}{ }^{\#}=\Delta \mathrm{H}_{\mathrm{A}}{ }^{\#}+\Delta \mathrm{H}$ zu überwinden.

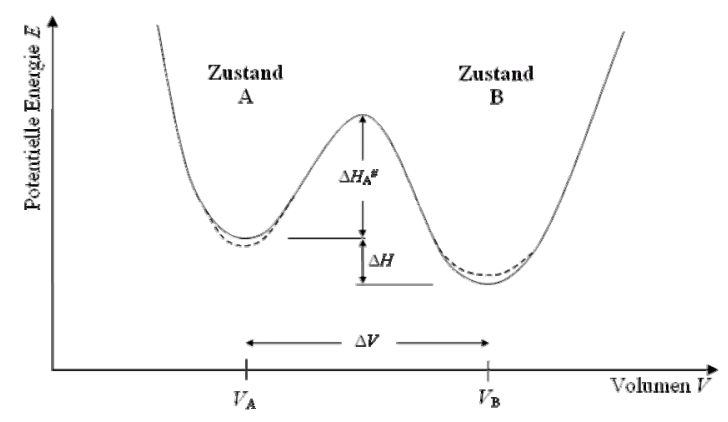

\section{Abbildung 4: Schematische Darstellung des Potentials eines Systems mit zwei möglichen Zuständen als Funktion des Volumens.}

Eine solche Gleichgewichtsreaktion kann durch die allgemeine Reaktionsgleichung

$$
\underbrace{\left|v_{1}\right| \cdot \mathrm{M}_{1}+\left|v_{2}\right| \cdot \mathrm{M}_{2}+\ldots+\left|v_{\mathrm{N}}\right| \cdot \mathrm{M}_{\mathrm{N}}}_{\text {Zustand } \mathrm{A}} \underset{k_{b}}{\stackrel{k_{f}}{\rightleftharpoons}} \underbrace{\left|v_{\mathrm{N}+1}\right| \cdot \mathrm{M}_{\mathrm{N}+1}+\left|v_{\mathrm{N}+2}\right| \cdot \mathrm{M}_{\mathrm{N}+2}+\ldots+\left|v_{\tilde{\mathrm{N}}}\right| \cdot \mathrm{M}_{\tilde{\mathrm{N}}}}_{\text {Zustand }}
$$

beschrieben werden, wobei $k_{f}$ und $k_{b}$ die Hin- bzw. Rückreaktionskonstante ${ }^{1}$ ist.

Für eine solche Reaktion gilt das Massenwirkungsgesetz

\footnotetext{
${ }^{1}$ Die Einheit der Reaktionskonstanten ist abhängig von der Ordnung der betrachteten Reaktion: $[k]=\mathrm{s}^{-1} \cdot(\mathrm{mol} / \mathrm{L})^{1-\mathrm{n}}$ für eine Reaktion $n$-ter Ordnung. Da bei einer Gleichgewichtsreaktion Hin- und Rückreaktion unterschiedlicher Ordnung $\left(n_{f}\right.$ bzw. $\left.n_{b}\right)$ sein können, kann auch der Fall $\left[k_{f}\right] \neq\left[k_{b}\right]$ auftreten. Für die Gleichgewichtskonstante $K$ gilt dementsprechend $[K]=(\mathrm{mol} / \mathrm{L})^{\Delta n}$ mit $\Delta n=n_{b}-n_{f}$. 


$$
K=\frac{k_{f}}{k_{b}}=\prod_{i}\left(c\left(\mathrm{M}_{i}\right)\right)^{v_{i}},
$$

wobei $K$ die Gleichgewichtskonstante der Reaktion ist und $c\left(\mathrm{M}_{i}\right)$ die Konzentration der Reaktanden $\mathrm{M}_{i}$ mit ihren stöchiometrischen Koeffizienten $v_{i}$ (nach Konvention ist dabei für die Edukte $v_{i}<0$ und für die Produkte $v_{i}>0$ ).

Als zwei typische Beispiele von Gleichgewichtsreaktionen sollen hier eine molekulare Konformationsänderung und ein Aggregations- oder Komplexierungsvorgang ${ }^{2}$ aufgezeigt werden. Im Falle einer molekularen Konformationsänderung, also wenn ein Molekül $\mathrm{M}$ in der Form $\mathrm{M}^{*}$ oder der Form $\mathrm{M}^{\#}$ vorliegen kann, werden die obigen Gleichungen zu

$$
\mathrm{M}^{*} \underset{k_{b}}{\stackrel{k_{f}}{\rightleftharpoons}} \mathrm{M}^{\#}
$$

und

$$
K=\frac{k_{f}}{k_{b}}=\frac{c\left(\mathrm{M}^{\#}\right)}{c\left(\mathrm{M}^{*}\right)} .
$$

Im anderen Fall, also einer Aggregation oder Komplexierung gemäß

$$
n \cdot \mathrm{N}+m \cdot \mathrm{M} \underset{k_{b}}{\stackrel{k_{f}}{\rightleftharpoons}}(n \cdot \mathrm{N} \circ m \cdot \mathrm{M})
$$

gilt für die Gleichgewichtskonstante

$$
K=\frac{k_{f}}{k_{b}}=\frac{c(n \cdot \mathrm{N} \circ m \cdot \mathrm{M})}{c^{n}(\mathrm{~N}) \cdot c^{m}(\mathrm{M})} .
$$

In Abbildung 4 ist durch die gestrichelte Linie bereits angedeutet, wie sich das Potential einer Gleichgewichtsreaktion schematisch verändert, wenn das betrachtete System einem äußeren Zwang ausgesetzt wird - im Beispiel der Abbildung wird durch eine Druckerhöhung das Gleichgewicht zugunsten jenes Zustands verschoben, der das geringere Volumen beansprucht, also Zustand A. Genau diese Verschiebung des Gleichgewichts wird beim klassischen Drucksprungexperiment (und auch bei anderen Sprungexperimenten) ausgenutzt. Dazu wird das System möglichst plötzlich einem Druck ausgesetzt oder ein bestehender Druck wird möglichst plötzlich abgebaut und dann wird anhand einer Messgröße, die für Zustand A und Zustand B verschiedene Werte annimmt, beobachtet, wie das System in den neuen Zustand übergeht.

${ }^{2}$ In dieser Arbeit wird der Begriff ,Komplex’ für eine Zusammenlagerung verschiedener Molekülsorten verwendet, während der Begriff, Aggregat' auf eine Zusammenlagerung von gleichen Molekülen (N=M) hindeuten soll. Beides wird allerdings in dieser Arbeit mit dem Symbol $\circ$ dargestellt. 
Da dieser Übergang in den neuen Gleichgewichtszustand auf thermischen Stößen beruht, kann er dem äußeren Zwang nicht instantan folgen, sondern das System relaxiert in den neuen Zustand mit einer systemspezifischen Relaxationszeit $\tau$ gemäß einer Differentialgleichung

$$
\frac{d Z}{d t}=-\frac{1}{\tau} \cdot\left(Z-Z_{\text {nеu }}\right)
$$

bzw. deren Lösung für $t \geq t_{0}$

$$
Z(t)=\left(Z_{\text {alt }}-Z_{\text {neu }}\right) \cdot e^{-\frac{t-t_{0}}{\tau}}+Z_{\text {neu }}
$$

In Abbildung 5 ist der schematische Verlauf des Drucks und der daraus resultierende Verlauf des Zustands $Z$ bzw. einer adäquaten ${ }^{3}$ Messgröße für ein solches Drucksprungexperiment aufgetragen. In der Abbildung ist bereits einer der begrenzenden Faktoren angedeutet, der es diesem Verfahren unmöglich macht, Relaxationszeiten zu bestimmen, die kleiner als $\sim 100 \mu$ s sind. Da $\delta t \ll \tau$ gelten muss und die Einstellzeit der Druckänderung, $\delta t$, aus technischen Gründen nicht beliebig klein gewählt werden kann, muss man sich für die Messung kleinerer Relaxationszeiten z.B. der Ultraschallabsorptionsspektroskopie bedienen.

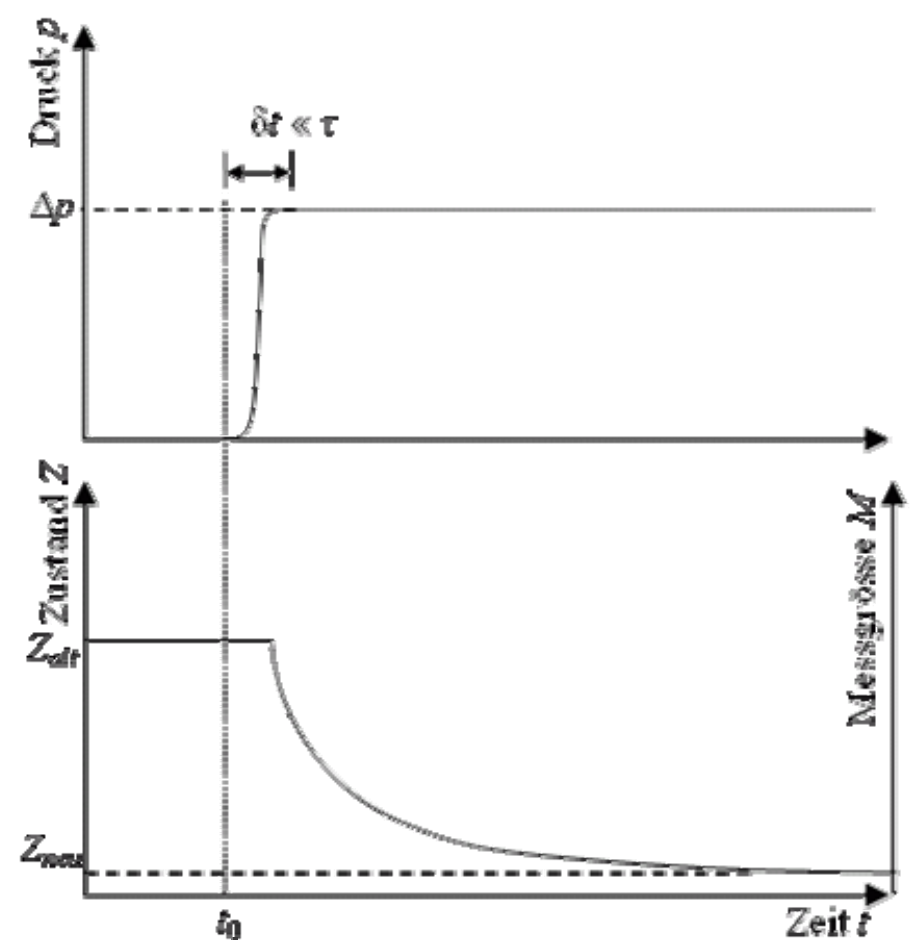

Abbildung 5: Schematische Darstellung des Verlaufs des Druckes $p$ (oben) sowie des Zustands $Z$ bzw. einer adäquaten ${ }^{3}$ Messgröße $M$ (unten) als Funktion der Zeit $t$ in einem Drucksprungexperiment.

\footnotetext{
${ }^{3}$ Adäquat ist in diesem Fall jede Größe, die für die beiden Zustände A und B verschieden ist und die schnell genug messbar ist. In der Praxis ist dies zumeist die Leitfähigkeit oder eine optische Größe. 
In der Ultraschallabsorptionsspektroskopie wird im Grunde ein periodisches Drucksprungexperiment durchgeführt - die eingestrahlte Schallwelle sorgt abwechselnd für eine kleine Auslenkung des Gleichgewichts durch Überdruck bzw. Unterdruck. Daher lässt sich bei zeitlich harmonischer Anregung für die Observable $Z$ in Differentialgleichung (2.14) ein komplexer Ansatz benutzen:

$$
Z(t)=\operatorname{Re}(Z)+i \cdot \operatorname{Im}(Z)=A \cdot e^{i \cdot \omega \cdot t}
$$

mit der Lösung [20]

$$
Z(t)=Z_{0} \cdot\left(1+\omega^{2} \tau^{2}\right)^{-1 / 2} \cdot \sin (\omega t-\arctan (\omega \tau))
$$

Hierbei sind $A$ und $Z_{0}$ zunächst beliebige Amplituden der Zustandsänderung. In Gl. (2.17) wird bereits deutlich, wie der Zustand des Systems vom Produkt aus der Kreisfrequenz der eingestrahlten Schallwelle, $\omega$, und der Relaxationszeit des betrachteten Prozesses, $\tau$, abhängt: Ist $\omega \cdot \tau \ll 1$, die Druckvariation also sehr viel langsamer als der Prozess, so kann das System immer in das neue Gleichgewicht relaxieren und die Phasenverschiebung arc$\tan (\omega \tau)$ zwischen Zustand $Z$ und dem Schalldruck $p$ ist vernachlässigbar klein. Ist hingegen $\omega \cdot \tau \gg 1$, die Druckvariation also sehr viel schneller als der Prozess, so kann das System der Schallwelle nicht folgen, der Term (1+ $\left.\omega^{2} \tau^{2}\right)^{-1 / 2}$ geht gegen Null und damit auch $Z(t)$. Entscheidend ist also der Fall $\omega \cdot \tau \approx 1$, wenn Druckvariation und Prozess etwa gleich schnell ablaufen. Dann ist der Zustand des Systems gegenüber der Schallwelle immer um $\pi / 4$ phasenverschoben und die Amplitude der Variation des Zustands beträgt $0.707 \cdot Z_{0}$.

Eine genaue Betrachtung der Energie, die das System durch die Phasenverschiebung von Zustand $Z$ und Druck $p$ von der Schallwelle aufnimmt, und der dadurch verursachten sogenannten Exzessdämpfung $\alpha_{e x z}$, (wiederum notiert in den gebräuchlichen Formen $\left(\alpha / f^{2}\right)$ bzw. $\left.(\alpha \lambda)\right)$ ergibt [20]:

$$
\begin{aligned}
& \left(\frac{\alpha}{f^{2}}\right)_{e x z}(\omega)=\frac{2 \cdot \pi^{2} \cdot c_{S} \cdot \rho \cdot \Gamma}{R \cdot T} \cdot\left(\Delta V-\frac{\gamma_{T}}{C_{p}} \cdot \Delta H\right)^{2} \cdot \frac{1}{1+\omega^{2} \tau^{2}} \\
& (\alpha \lambda)_{e x z}(\omega)=\frac{\pi \cdot c_{S}^{2} \cdot \rho \cdot \Gamma}{R \cdot T} \cdot\left(\Delta V-\frac{\gamma_{T}}{C_{p}} \cdot \Delta H\right)^{2} \cdot \frac{\omega \tau}{1+\omega^{2} \tau^{2}}
\end{aligned}
$$

Diese sogenannte Debye-Spektralfunktion beschreibt also die zusätzliche Dämpfung, die ein molekularer Prozess mit diskreter Relaxationszeit $\tau$ verursacht. Die Amplitude der Debye-Spektralfunktion

$$
A_{D}=\frac{\pi \cdot c_{S}^{2} \cdot \rho \cdot \Gamma}{R \cdot T} \cdot\left(\Delta V-\frac{\gamma_{T}}{C_{p}} \cdot \Delta H\right)^{2}
$$

beinhaltet dabei sowohl Größen, die von dem Medium abhängen, in dem der Prozess stattfindet (Schallgeschwindigkeit $c_{S}$, Dichte $\rho$, Temperatur $T$, thermischer Ausdehnungskoeffizient $\gamma_{T}$ und spezifische isobare Wärmekapazität $C_{p}$ ), als auch Größen, die von dem betrachteten Prozess abhängen (Reaktionsvolumen $\Delta V$, Reaktionsenthalpie $\Delta H$ und Stöchiometriefaktor $\Gamma$ ). 
Der Stöchiometriefaktor $\Gamma$ ist dabei definiert als

$$
\Gamma \equiv\left(\sum_{i} \frac{v_{i}}{c\left(\mathrm{M}_{i}\right)}\right)^{-1}
$$

mit den stöchiometrischen Koeffizienten $v_{i}$ und den Konzentrationen $c\left(\mathrm{M}_{i}\right)$ der beteiligten Spezies.

Die frequenzabhängige Dämpfung einer Flüssigkeit setzt sich nun additiv aus den beschriebenen Anteilen $\alpha_{v i s k}$, $\alpha_{\text {therm }}$ und $\alpha_{e x z}$ zusammen, wobei die Exzessdämpfung keineswegs nur auf einem einzigen molekularen Prozess beruhen muss, sondern aus $N$ verschiedenen Einzelzeitrelaxationen bestehen kann:

$$
\begin{aligned}
(\alpha \lambda)_{\text {ges }}(\omega) & =(\alpha \lambda)_{\text {exz }}+(\alpha \lambda)_{\text {visk }}+(\alpha \lambda)_{\text {therm }} \\
& =\sum_{i=1}^{N} \frac{A_{D}^{(i)} \cdot \omega \cdot \tau_{i}}{1+\omega^{2} \cdot \tau_{i}^{2}}+B_{\text {theo }} \cdot f \\
& =\sum_{i=1}^{N_{\text {prakt }}} \frac{A_{D}^{(i)} \cdot \omega \cdot \tau_{i}}{1+\omega^{2} \cdot \tau_{i}^{2}}+B_{\text {prakt }} \cdot f \\
\left(\frac{\alpha}{f^{2}}\right)_{\text {ges }}(\omega) & =\left(\frac{\alpha}{f^{2}}\right)_{\text {exz }}+\left(\frac{\alpha}{f^{2}}\right)_{\text {visk }}+\left(\frac{\alpha}{f^{2}}\right)_{\text {therm }} \\
& =\frac{2 \cdot \pi}{c_{S}} \cdot \sum_{i=1}^{N} \frac{A_{D}^{(i)}}{1+\omega^{2} \cdot \tau_{i}^{2}}+\frac{B_{\text {theo }}}{c_{S}} \\
& =\frac{2 \cdot \pi}{c_{S}} \cdot \sum_{i=1}^{N_{\text {prakt }}} \frac{A_{D}^{(i)}}{1+\omega^{2} \cdot \tau_{i}^{2}}+\frac{B_{\text {prakt }}}{c_{S}} .
\end{aligned}
$$

In Gl. (2.21) ist bereits der Tatsache Rechnung getragen, dass möglicherweise messtechnisch nicht alle $N$ in dem Medium auftretenden Relaxationsprozesse aufgenommen werden können, sondern nur die $N_{\text {prakk }}$, die im Messbereich liegen. Daher definiert man den Hintergrundsanteil $B$ so, dass er neben dem theoretischen Wert $B_{\text {theo }}$ gemäß Gl. (2.6) auch alle Relaxationsanteile $N_{\text {prakt }}+1 \ldots N$ enthält, die oberhalb des Messbereiches liegen.

In Abbildung 6 ist eine Debye-Spektralfunktion in den Formen $\left(\alpha / f^{2}\right)(\omega \cdot \tau)$ bzw. $(\alpha \lambda)_{e x z}(\omega \cdot \tau)$ dargestellt. In der $(\alpha \lambda)_{e x z}$-Darstellung äußert sich ein mit einer Debye-Spektralfunktion beschreibbarer molekularer Prozess als ein Maximum bei $\left[\omega \cdot \tau=1 ; 0.5 \cdot A_{D}\right]$ und in $\operatorname{der}\left(\alpha / f^{2}\right)$-Darstellung als monoton fallende Funktion zwischen den beiden Plateaus $\left(2 \cdot \pi \cdot A_{D}+B\right) / c_{S}$ für $\omega \cdot \tau \ll 1$ und $B / c_{S}$ für $\omega \cdot \tau » 1$.

Aus der Anpassung einer Funktion nach Gl. (2.21) an ein Ultraschallabsorptionsspektrum lassen sich also $2 \cdot N+1$ Parameter gewinnen, nämlich $N$ Relaxationszeiten $\tau_{i}, N$ Amplituden $A_{D, i}$ und der $B$-Wert. Aus dem $B$-Wert lassen sich im Allgemeinen keine weiteren Informationen gewinnen, da er, wie bereits erwähnt, neben dem theoretischen Anteil gemäß Gl. (2.6) auch noch Anteile von höherfrequenten Relaxationen beinhalten kann. 

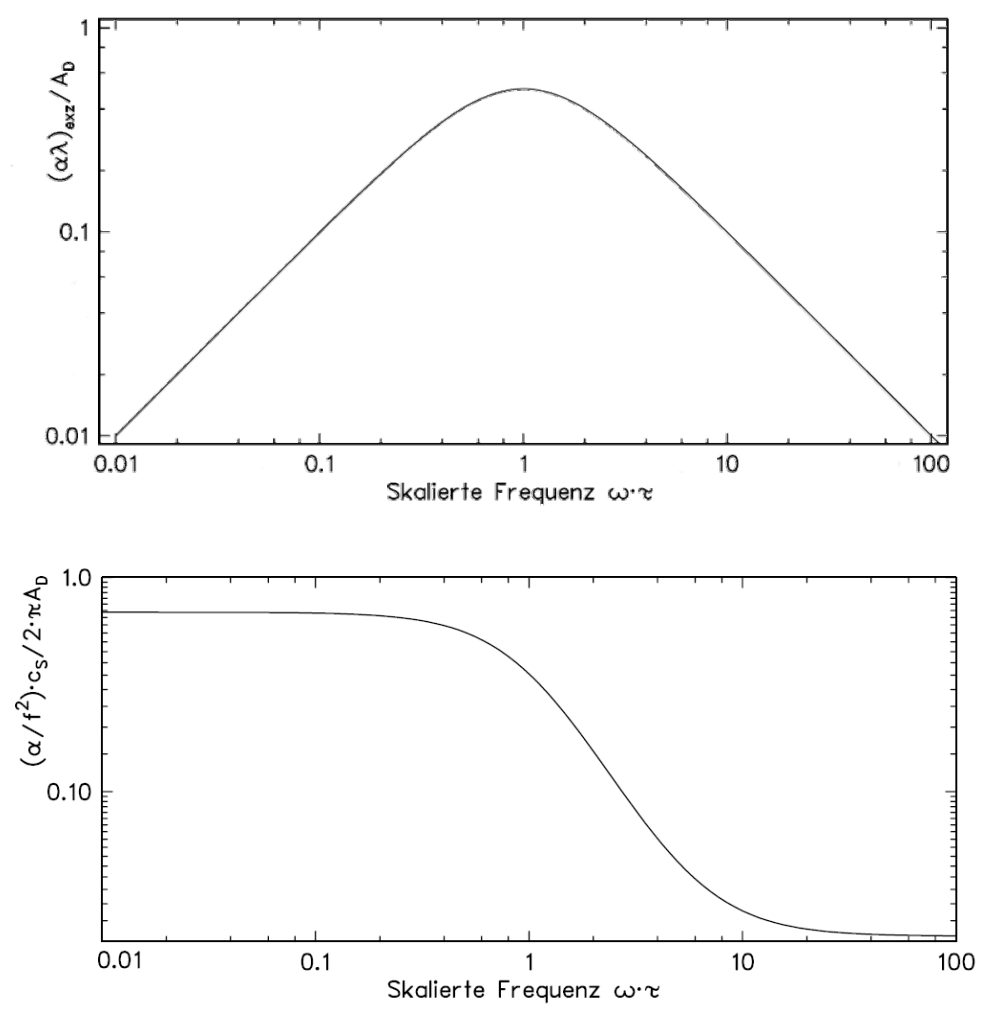

\section{Abbildung 6: Darstellung der Debye-Spektralfunktionen in den beiden gebräuchlichen Formen $(\alpha \lambda)_{e x z}\left(\right.$ oben) und $\left(\alpha / f^{2}\right)$ (unten) als Funktion einer skalierten Frequenz $(\omega \cdot \tau)$.}

Aus der Amplitude $A_{D}$ einer Debye-Spektralfunktion kann hingegen bei Kenntnis der anderen Größen in Gl. (2.19) das isentropische Reaktionsvolumen $\Delta V_{S}$

$$
\Delta V_{S}=\Delta V-\frac{\gamma_{T}}{C_{p}} \cdot \Delta H
$$

bestimmt werden. Eine Bestimmung des isothermen Reaktionsvolumens $\Delta V$ ist ohne genaue Kenntnis der Reaktionsenthalpie $\Delta H$ nicht möglich, allerdings ist bei den in der Ultraschallabsorptionsspektroskopie messbaren Prozessen im Allgemeinen $\Delta H \cdot \gamma_{T} / C_{p} \ll \Delta \mathrm{V}$, so dass $\Delta V_{S} \approx \Delta V$ ist.

Weiterhin lassen sich aus den Relaxationszeiten $\tau$ zum einen Schlüsse über die Lebensdauer eines Zustandes treffen und zum anderen können gemäß [20]

$$
\tau^{-1}=k_{f} \cdot \sum_{i} c^{\left|v_{i}\right|}\left(\mathrm{A}_{i}\right)+k_{b} \cdot \sum_{j} c^{\left|v_{j}\right|}\left(\mathrm{B}_{j}\right)
$$

Informationen über die Reaktionskonstanten $k_{f}$ und $k_{b}$ gewonnen werden.

Für die beiden oben angeführten Beispiele molekularer Prozesse, der Konformationsänderung und der Aggregation/Komplexierung, ergeben sich dann konkret folgende Zusammenhänge: 


$$
\Gamma=\left(c^{-1}\left(\mathrm{M}^{*}\right)+c^{-1}\left(\mathrm{M}^{\#}\right)\right)^{-1}=c(\mathrm{M}) \cdot\left(p\left(\mathrm{M}^{*}\right)^{-1}+p\left(\mathrm{M}^{\#}\right)^{-1}\right)^{-1}
$$

und

$$
\tau^{-1}=k_{f}+k_{b}
$$

für die Konformationsänderung. Hierbei beschreibt $p\left(\mathrm{M}^{*}\right)$ den Anteil der Moleküle im Zustand $\mathrm{M}^{*}$ und $p\left(\mathrm{M}^{\#}\right)$ den Anteil im Zustand $\mathrm{M}^{\#}$.

Für einen Aggregations-/Komplexierungsvorgang hingegen ergibt sich

$$
\Gamma=\left((n \cdot c(\mathrm{~N}))^{-1}+(m \cdot c(\mathrm{M}))^{-1}+(c(n \cdot \mathrm{N} \circ m \cdot \mathrm{M}))^{-1}\right)^{-1}
$$

und

$$
\tau^{-1}=k_{f} \cdot\left(c^{n}(\mathrm{~N})+c^{m}(\mathrm{M})\right)+k_{b}
$$

Im Falle der Konformationsänderungen ist der Stöchiometriefaktor $\Gamma$ nach Gl. (2.24) proportional zur Gesamtkonzentration $c(\mathrm{M})$. Für die spätere Analyse wird es zweckmäßig sein, den Proportionalitätsfaktor zu definieren:

$$
\Sigma \equiv \frac{\Gamma}{c(\mathrm{M})}=\left(p\left(\mathrm{M}^{*}\right)^{-1}+p\left(\mathrm{M}^{\#}\right)^{-1}\right)^{-1}
$$

Diese Größe ist dann also nur noch von den Anteilen der Moleküle in den möglichen Zuständen abhängig, also der Verteilung der Konformationspopulationen. Bei einer Konformationsänderung, die zwei mögliche Zustände aufweist, ergibt sich bei einer gleichmäßigen Verteilung, also $p\left(\mathrm{M}^{*}\right)=p\left(\mathrm{M}^{\sharp}\right)=0.5$, für diese Größe ein Wert von $\Sigma=\left(0.5^{-1}+0.5^{-1}\right)^{-1}=0.25$. Dies ist der maximale Wert, den die Größe $\Sigma$ annehmen kann - eine ungleichmäßigere Verteilung (z.B. $\left.\Sigma=\left(0.75^{-1}+0.25^{-1}\right)^{-1}=0.19\right)$ oder eine größere Anzahl möglicher Konformationszustände (z.B. $\left.\Sigma=\left(0.33^{-1}+0.33^{-1}+0.33^{-1}\right)^{-1}=0.11\right)$ führen zu kleineren Werten. 


\title{
2.4 Das Ultraschalltransmissionsverfahren
}

Beim sogenannten Ultraschalltransmissionsverfahren werden zwei elektroakustische Schallwandler in einem veränderbaren Abstand voneinander positioniert um direkt den Schalldruckabfall gemäß Gl. (2.2) über der Strecke $x$ zu messen. Dazu wird der Sendewandler mit einer Wechselspannung seiner Grundresonanzfrequenz oder einer ihrer ungradzahligen Harmonischen angeregt und das Signal nach Durchlaufen der Flüssigkeit bei der gleichen Frequenz wieder aufgenommen. Um den Einfluss von Mehrfachreflexionen zwischen den Wandlern zu vermeiden, wird das Signal gepulst, wobei für die Länge $\tau_{P u l s}$ des Pulses gelten muss $\tau_{P u l s}<2 \cdot x / c_{S}$. Aus technischen Gründen kann $\tau_{\text {Puls }}$ allerdings nicht beliebig klein gewählt werden - zum einen können elektronisch nicht beliebig kleine Schaltzeiten realisiert werden, zum anderen sieht das Frequenzspektrum einer gepulsten Schwingung bekanntlich aus wie das Beugungsbild eines Spaltes, wobei die Halbwertsbreite des Hauptmaximums umgekehrt proportional zur Pulsdauer ist: $\Delta f \cdot \tau_{P u l s} \approx 1$ [21]. Um möglichst alle Anteile der einfallenden Schwingung aufnehmen zu können, wird der Empfänger daher mit einer gewissen Bandbreite $\Delta f$ betrieben, die aber wegen der damit verbundenen Verschlechterung des Signal/Rausch-Verhältnisses nicht beliebig vergrößert werden kann.

In Abbildung 7 sind schematisch das Ultraschalltransmissionsverfahren (links) und ein gepulstes Sinussignal (rechts) dargestellt.
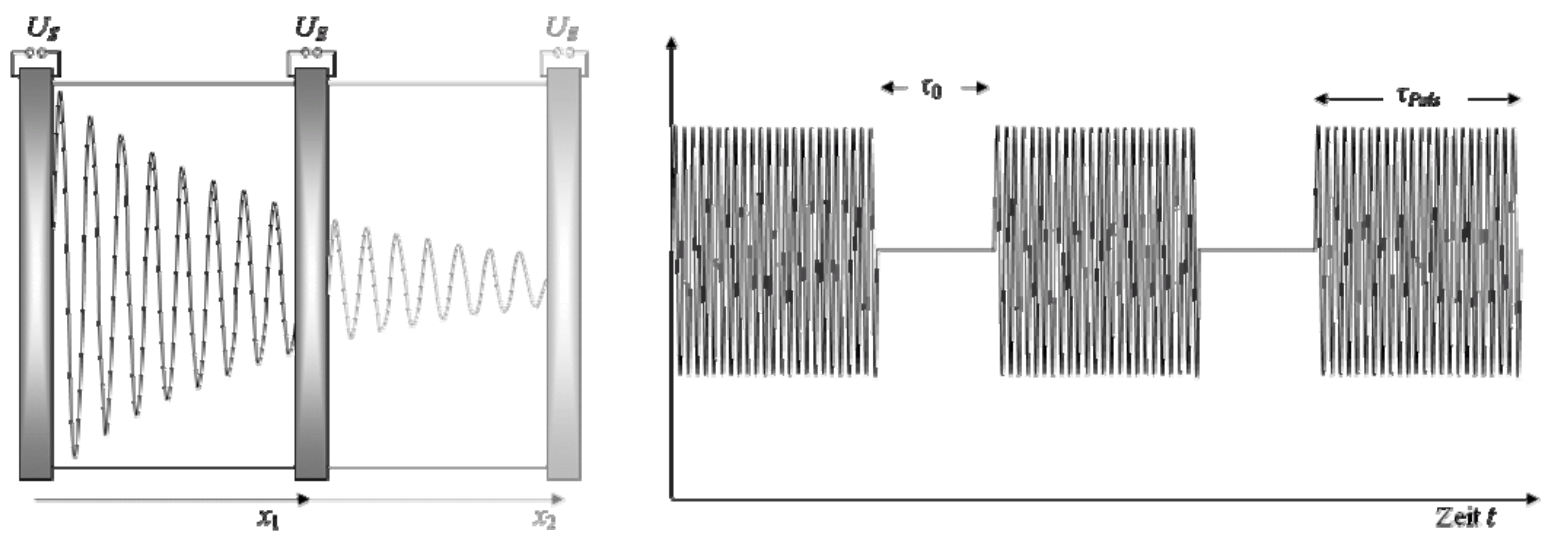

\begin{abstract}
Abbildung 7: Schematische Darstellung des Ultraschalltransmissionsverfahrens (links) und einer gepulsten Schwingung (rechts). Im linken Bild sind der Sendewandler mit der Sendespannung $U_{S}$ (links) und der Empfangswandler mit der Empfangsspannung $U_{E}$ (Mitte bzw. rechts) in zwei möglichen Abständen $x_{1}$ und $x_{2}$ dargestellt.
\end{abstract}

Die Tatsache, dass der Hintergrundsanteil des Dämpfungsexponenten gemäß Gl. (2.5) proportional zu $f^{2}$ ansteigt, ist dabei der maßgeblich begrenzende Faktor bei der Frage, innerhalb welchen Frequenzbereichs mit einer Ultraschalltransmissionsapparatur gemessen werden kann. Berechnet man zum Beispiel für Wasser den Weg, auf dem der Schalldruck auf die Hälfte abnimmt (als das Kriterium für eine signifikante Abnahme), so erhält man mit den aus der Literatur bekannten Dämpfungswerten $\alpha / f^{2}=21.28 \cdot 10^{-15} \mathrm{~s}^{2} / \mathrm{m}$ bzw. $B=31.80$ ps [22] die in Tabelle 2.1 aufgelisteten Messstrecken $x$ bei einigen angestrebten Frequenzen. 


\begin{tabular}{r|r}
\hline \hline Frequenz $f[\mathrm{MHz}]$ & Strecke $x[\mathrm{~m}]$ \\
\hline \hline 0.1 & $3.3 \cdot 10^{3}$ \\
\hline 1 & 33 \\
\hline 10 & 0.33 \\
\hline 100 & $3.3 \cdot 10^{-3}$ \\
\hline 1000 & $3.3 \cdot 10^{-5}$ \\
\hline \hline
\end{tabular}

Tabelle 2.1: Berechnung der Wegstrecke $x$, auf der für die gegebene Frequenz $f$ der Schalldruck in Wasser auf die Hälfte abfällt.

Während bei kleinen Frequenzen $(f<10 \mathrm{MHz})$ offensichtlich im Labor kaum zu realisierende Messstrecken benötigt würden, sind die Messstrecken für höhere Frequenzen (10 MHz $<f<1 \mathrm{GHz})$ zwar durchaus realisierbar, allerdings nicht mit einer einzigen Apparatur. In der vorliegenden Arbeit wurde daher mit zwei verschiedenen Ultraschalltransmissionsapparaturen gemessen - einer tieffrequenten Apparatur für den Bereich $10 \mathrm{MHz}<f$ $<80 \mathrm{MHz}$ und einer hochfrequenten Apparatur für den Bereich $30 \mathrm{MHz}<f<400 \mathrm{MHz}$.

In Abbildung 9 ist die tieffrequente Messzelle schematisch dargestellt, die sich von der benutzten hochfrequenten Messzelle vor allem in den Abmessungen und in einigen Details unterscheidet. So wurde bei der tieffrequenten Zelle eine Zellberandung (3) aus Plexiglas benutzt, während bei der hochfrequenten Zelle eine Berandung aus Stahl zum Einsatz kam. Weiterhin waren bei der hochfrequenten Messzelle Verzögerungsleitungen (siehe unten) eingebaut.

Für eine Messung mit einer Ultraschalltransmissionszelle wird die zu untersuchende Lösung im Ultraschallbad sorgfältig entgast und dann über die Zu-/Ablaufkanüle (19) in die Zelle gegeben. Ein Thermostat pumpt Wasser mit der gewünschten Messtemperatur durch die Thermostatierkanäle (3), (17) um die gesamte Apparatur auf die Messtemperatur zu bringen. Zusätzlich befindet sich die gesamte Messzelle in einer Außenhülle, die von einem zweiten unabhängigen Thermostatierkreislauf temperiert wird. Mit diesem Verfahren wird eine Temperaturstabilität von $\Delta T \leq 0.02 \mathrm{~K}$ erreicht. Die Temperatur wird über einen vorher kalibrierten Temperaturfühler ausgelesen, der sich am Rande des Messvolumens (1) in der Zellberandung (3) befindet.

Nach ausreichend langer Thermostatierzeit werden bei jeder Messfrequenz drei Messungen durchgeführt, indem die Übertragungsfunktion $T(x)$ an 400 Stützstellen über eine vorher zu bestimmende Messstrecke gemessen wird. Die Übertragungsfunktion $T(x)$ gibt das Verhältnis von Empfangsspannung $U_{E}$ und Sendespannung $U_{S}$ wieder und ist proportional zum Verhältnis der Schalldrücke am Empfangs- bzw. Sendewandler und damit auch proportional zum exponentiellen Abfall in Gl. (2.2):

$$
T(x)=\frac{U_{E}(x)}{U_{S}(x)} \sim e^{-\alpha \cdot x} \cdot\left(e^{-\sqrt{\frac{x}{k \cdot A}}}\right)^{g(x)}
$$

Hier ist bereits durch den zweiten Faktor eine Beugungskorrektur nach Menzel [23] eingebaut, die berücksichtigt, dass durch die endlichen Wandlerradien nicht der gesamte Schall als ebene Welle parallel zur Wandleroberfläche abgestrahlt wird, sondern es am Rand der Wandler zu Beugungsverlusten kommt. Dabei ist $k$ die Wellen- 
zahl der Schallwelle, $A$ die Fläche der Wandler und $g(x)$ eine empirische Korrekturfunktion, die für die benutzte tieffrequente Messzelle die Form $g(x)=1-x / 100 \mathrm{~mm}$ hat.

Eine weitere nötige Korrektur ergibt sich durch das sogenannte elektrische Übersprechen, das daher resultiert, dass an den Wandlern keine vollständige Umwandlung von elektrischer Energie in Schallenergie (bzw. umgekehrt) erfolgt. Daher geht ein Teil des Signals direkt als elektromagnetische Welle vom Sendewandler zum Schallwandler über. Dies wirft zwei Probleme auf: Zum einen fehlt dieser im Allgemeinen nicht bekannte Anteil der Energie, wenn versucht wird den exakten Schalldruck an Sende- und Empfangswandler zu berechnen. Da allerdings in Gl. (2.29) nur eine Proportionalität verlangt wird, spielt dies in der Praxis keine Rolle. Zum anderen stellt aber eine zeitliche Überlagerung von elektrischem Signal und Schallsignal am Empfangswandler eine Verfälschung des Messwertes dar. Bei großen Messstrecken ergibt sich eine zeitliche Trennung der beiden Signale automatisch durch die sehr unterschiedlichen Laufzeiten von elektromagnetischen Wellen und Schall-Wellen, bei kleinen Messstrecken hingegen wird ein solcher Laufzeitenunterschied dadurch erzeugt, dass sogenannte Verzögerungsleitungen in den Signalweg eingebaut werden. In der hochfrequenten Pulszelle ist dies durch akustisch möglichst verlustfrei angekoppelte Quarz-Stäbe realisiert, die sich jeweils zwischen Wandler und Messflüssigkeit befinden.

Da die am Empfangswandler aufgenommene Spannung erst nach diversen elektronischen Verfahren (Mischen, Demodulation, Verstärkung etc., siehe Abbildung 8) digital bestimmt wird, die nicht notwendigerweise alle linear sind, muss, um aus den am PC aufgenommenen Spannungswerten $U_{\text {Mess }}$ die wahren Spannungen $U_{\text {Wahr }}$ berechnen zu können, eine Kennlinie des gesamten elektronischen Systems aufgenommen werden. Dazu wird nach jeder einzelnen Messung eine Referenzmessung mithilfe eines Cut-Off-Dämpfungsglieds vorgenommen, das aus einem Hohlleiter besteht, der unterhalb seiner Grenzfrequenz betrieben wird. Da für einen solchen Hohlleiter die Spannung in Abhängigkeit von seiner Länge wohlbekannt ist [24], kann so $U_{\text {Wahr }}\left(U_{\text {Mess }}\right)$ für alle Spannungsmesswerte bestimmt werden.

Wie weiter oben bereits erwähnt, kann die Pulsdauer $\tau_{P u l s}$ der einhüllenden Pulsfunktion aus elektronischen Gründen nicht beliebig klein gewählt werden, so dass die Bedingung $\tau_{P u l s}<2 \cdot x / c_{S}$, die die Trennung des Primärsignals von seinen Echos gewährleistet, nicht immer erfüllt werden kann. Daher kann es bei kleinen Startabständen $x$ zu einer Welligkeit des Signals kommen, die auf den Überlagerungen des Primärsignals und seiner Reflexionen beruht. Da allerdings in den verwendeten Lösungen wegen der geringen Konzentrationen vergleichsweise geringe Dämpfungen auftraten, konnten im Allgemeinen hinreichend große Startabstände gewählt werden, so dass diese Welligkeit des Signals vermieden werden konnte.

Der Messfehler bei den Ultraschalltransmissionsapparaturen kann durch Mehrfachmessungen auf $\Delta \alpha / \alpha<2 \%$ abgeschätzt werden. Lediglich bei tiefen Frequenzen muss ein größerer Fehler angenommen werden, da unterhalb von ca. $20 \mathrm{MHz}$ bei der tieffrequenten Messzelle, bzw. ca. $50 \mathrm{MHz}$ bei der hochfrequenten Zelle, wegen der geringen Hintergrundsdämpfung nicht mehr der erstrebte signifikante Signalabfall von $15 \mathrm{~dB}$ erreicht werden konnte, sondern wegen der begrenzten Messstrecke kleinere Signalabfälle auftraten. In der Praxis erwiesen sich allerdings auch die Messungen in diesen Frequenzbereichen als gut reproduzierbar. 


\begin{tabular}{c|c|c}
\hline \hline & Tieffreq. Messzelle & Hochfreq. Messzelle \\
\hline \hline Wandlermaterial & Quarz & Quarz \\
\hline Verzögerungsleitungen & nein & Ja \\
\hline Wandlerradius $r_{Q}[\mathrm{~mm}]$ & 20 & 6 \\
\hline Wandlergrundresonanzfrequenz $f_{Q}[\mathrm{MHz}]$ & 1.05 & 10.8 \\
\hline Maximale Messfrequenz $f_{\text {Max }}[\mathrm{MHz}]$ & 100 & 530 \\
\hline Pulsdauer $\tau_{P u l s}[\mu \mathrm{s}]$ & $4-8$ & $2-4$ \\
\hline Längentaster & Haidenhain MT60 & Haidenhain MT25 \\
\hline Minimaler Wandlerabstand $x_{\text {Min }}[\mathrm{nm}]$ & 125 & 125 \\
\hline Maximaler Wandlerabstand $x_{\text {Max }}[\mathrm{mm}]$ & 55 & 25 \\
\hline Messvolumen $V[\mathrm{ml}]$ & $\approx 130$ & $\approx 10$ \\
\hline Literatur & {$[25]$} & {$[26]$} \\
\hline \hline
\end{tabular}

Tabelle 2.2: Parameter der beiden benutzten Ultraschalltransmissionsmesszellen.

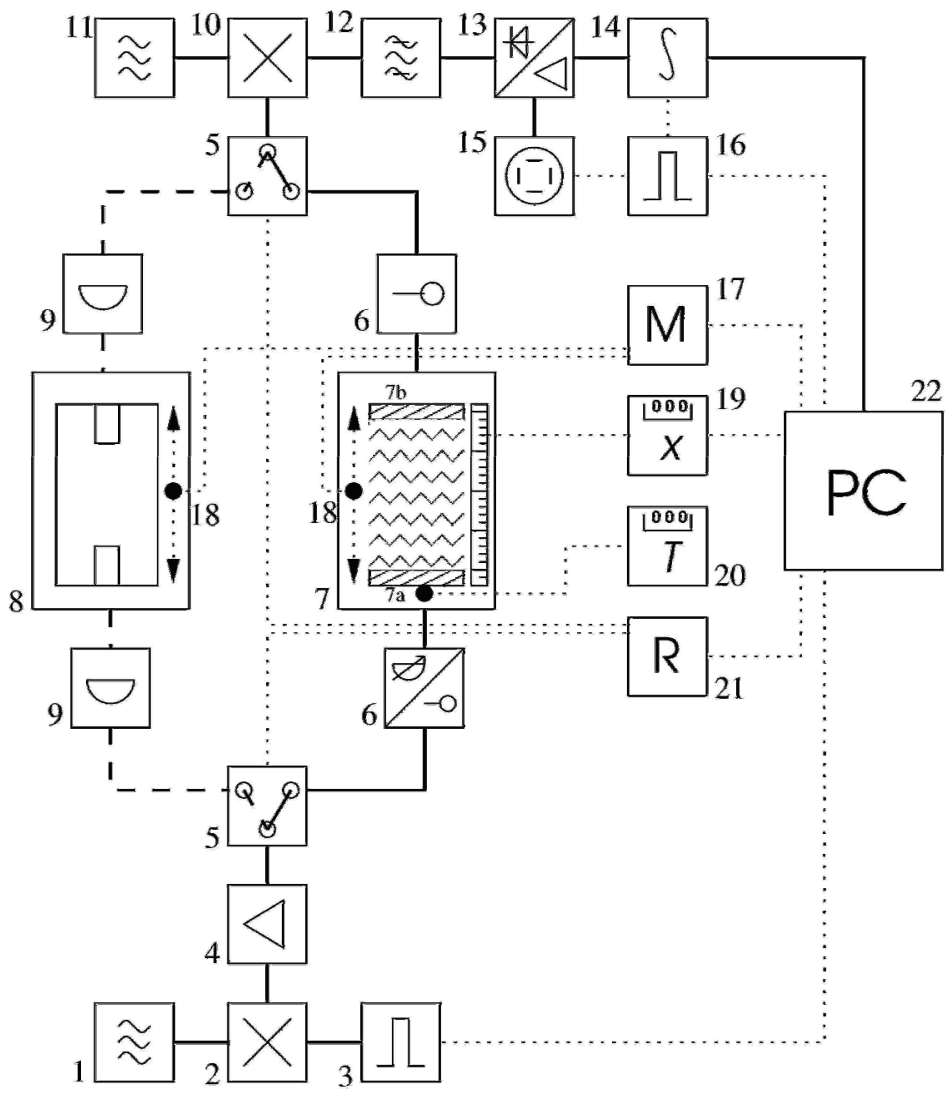

Abbildung 8: Blockschaltbild einer Ultraschalltransmissionsmessapparatur: Das vom Synthetisiersender (1) erzeugte Signal wird im Modulator (2) mit dem Puls aus dem Pulsgenerator (3) moduliert, in einem Leistungsverstärker (4) verstärkt und gelangt über ein Koaxialrelais (5) entweder in den Messzweig (rechts) oder den Referenzzweig (links). Dort durchläuft es ein variables Dämpfungsglied (6), die Messzelle (7) mit Sende- (7a) und Empfangswandler (7b) bzw. zwei Anpassungsdämpfungsglieder (9) und das Cut-Off-Dämpfungsglied (8). Anschließend gelangt das Signal über den Überlagerungsempfänger mit Mischer (10), Hilfsoszillator (11), Zwischenfrequenzfilter (12) und Demodulator mit Verstärker (13) in das Oszilloskop (15), bzw. über Sample-and-Hold und A/D-Wandler (14) und Pulsgenerator (16) in den Rechner (22). Die Schrittmotoren (18) werden vom PC über die Schrittmotorsteuerung (17) betrieben, der auch den Längentaster (19) ausliest und per Relaistreiberkarte (21) die Koaxialrelais schaltet. Die Temperatur wird vom Temperaturfühler (20) erfasst. In der gesamten Abbildung bedeuten durchgezogene und gestrichelte Linien eine Signalleitung, gepunktete Linien eine Rechnersteuerungsleitung. 


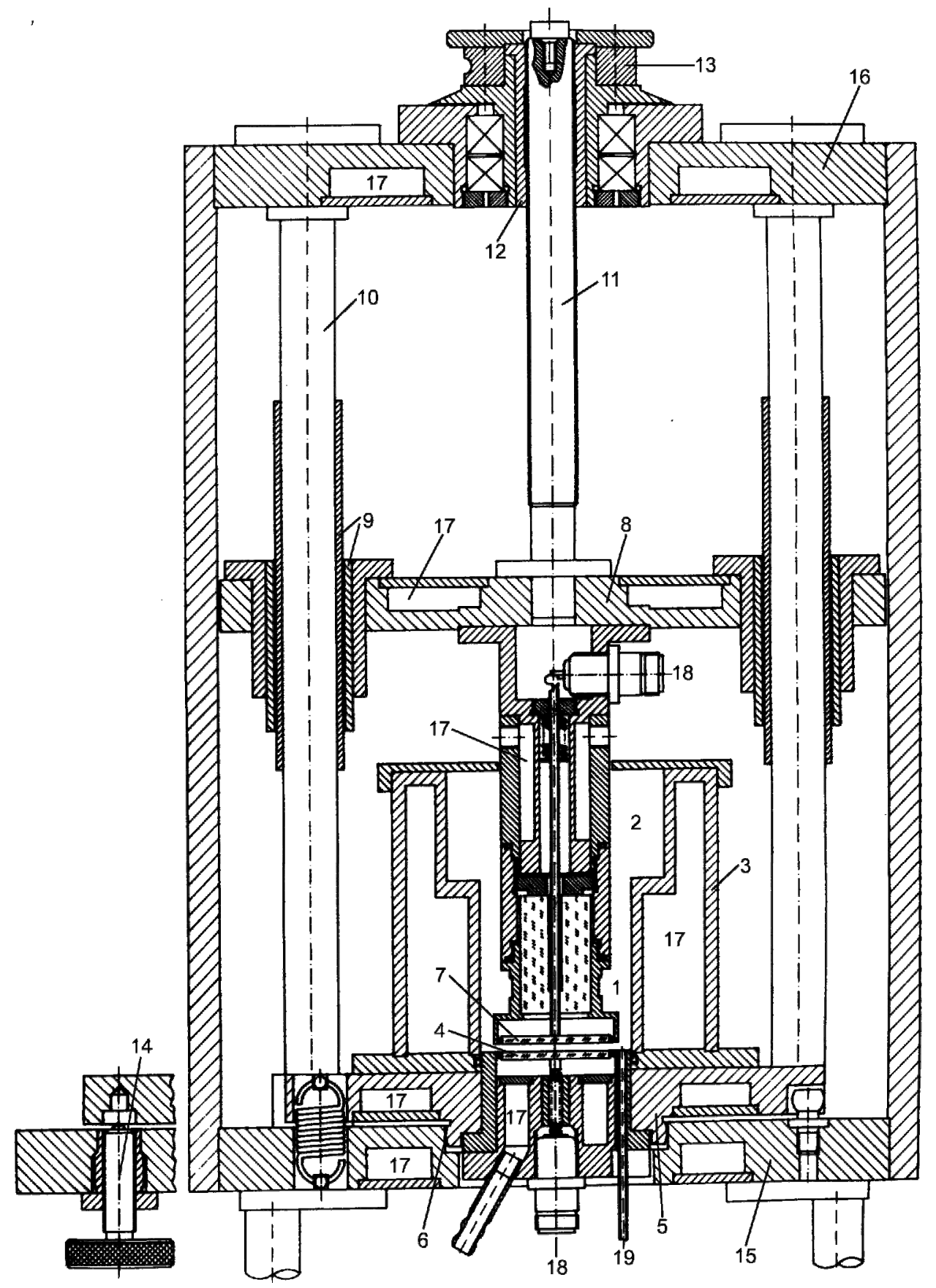

Abbildung 9: Schematische Darstellung einer Ultraschalltransmissionszelle: Messvolumen (1) mit Vorratsbereich (2), Thermostatiermantel (3), Sende- (4) und Empfangswandler (7), kippbare Montageplatte (5) auf einem Kugelgelenk (6), verschiebbare Montageplatte (8) mit Kugelführung (9), Führungsstange (10), Spindel (11), Mutter (12) und Riemenscheibe (13) für den Antrieb von (11), Schrauben zur Paralleljustierung (14), Grund- (15) und Deckenplatte (16), Thermostatierkanal (17), N-Norm-Anschluss (18) und Zu-/Ablaufkanüle (19). 


\subsection{Das Resonatorverfahren}

Die Berechnungen in Tabelle 2.1 zeigen, dass bei Frequenzen unterhalb von etwa $10 \mathrm{MHz}$ für das Ultraschalltransmissionsverfahren Messstrecken vonnöten wären, die im Laboralltag nicht zu realisieren wären. Zum einen würden solch große Messstrecken sehr große und damit teure Messvolumina bedeuten, zum anderen wäre die Thermostatierung dieser großen Volumina mit erheblichem Aufwand verbunden. Daher bedient man sich unterhalb von etwa $10 \mathrm{MHz}$ des Resonatorverfahrens, bei dem der Weg des Schalles nach dem Resonanzprinzip zwischen zwei feststehenden Wandlern vervielfacht wird.

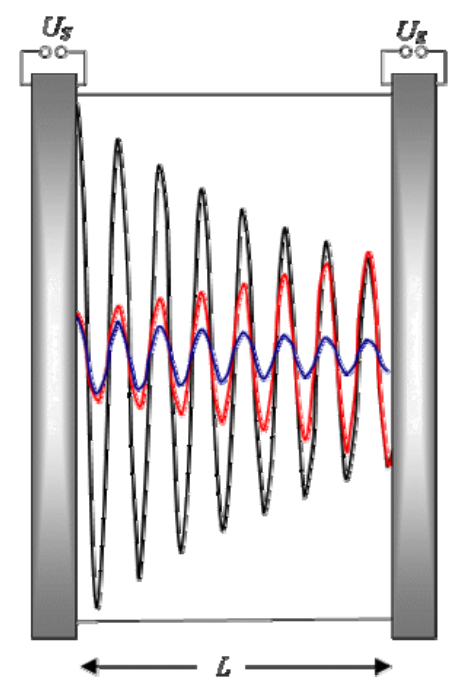

Abbildung 10: Schematische Darstellung des Resonatorprinzips. Links ist der Sendewandler mit der Sendespannung $U_{S}$ angedeutet, rechts der Empfangswandler mit der Empfangsspannung $U_{E}$ und dazwischen das Messvolumen, in dem eine Schallwelle (schwarz) und die erste (rot) bzw. zweite (blau) Reflexion angedeutet sind.

Setzt man zunächst einmal lateral unendlich ausgedehnte Wandler voraus, lässt sich der Schalldruck am Empfangswandler durch eine einfache geometrische Reihe berechnen:

$$
\begin{aligned}
p_{E}= & p_{S}^{0} \cdot(1+r) \cdot e^{-\gamma L} \cdot e^{i \omega t} \\
& +p_{S}^{0} \cdot r^{2} \cdot(1+r) \cdot e^{-3 \gamma L} \cdot e^{i \omega t} \\
& +p_{S}^{0} \cdot r^{4} \cdot(1+r) \cdot e^{-5 \gamma L} \cdot e^{i \omega t} \\
& +\ldots \\
= & p_{S}^{0} \cdot(1+r) \cdot e^{-\gamma L} \cdot e^{i \omega t} \cdot \sum_{n=0}^{\infty}\left(r^{2} \cdot e^{-2 \gamma L}\right)^{n} \\
= & \frac{p_{S}^{0} \cdot(1+r) \cdot e^{-\gamma L} \cdot e^{i \omega t}}{1-r^{2} \cdot e^{-2 \gamma L}}
\end{aligned}
$$

Hierbei ist $p_{E}$ der Schalldruck am Empfangswandler, $p_{S}{ }^{0}$ die Schalldruckamplitude der eingestrahlten Schallwelle, $L$ der Abstand zwischen Sende- und Empfangswandler, $r$ der Reflexionsfaktor am Übergang FlüssigkeitWandler, $\gamma=\alpha+i \cdot k$ die komplexe Ausbreitungskonstante mit dem Dämpfungsexponenten $\alpha$, der Wellenzahl $k=2 \pi f / c_{S}$ sowie der Schallgeschwindigkeit $c_{S}$. 
Nimmt man weiterhin an, dass $|r|=1$ (schallharte Reflexion), so lässt sich aus Gl. (2.30) die Übertragungsfunktion $T(f)$ berechnen:

$$
\begin{aligned}
T(f)=\frac{U_{E}}{U_{S}} \sim \frac{1}{e^{\gamma L}-e^{-\gamma L}} & =\frac{1}{2 \cdot \sinh (\gamma L)} \\
& =\frac{1}{\sqrt{\sinh ^{2}(\alpha L)+\sin ^{2}(k L)}}
\end{aligned}
$$

Damit erhält man unter den obigen Annahmen die bekannte Resonanzbedingung für ideale Resonatoren, die in der Praxis bei genauer Kenntnis der Zelllänge $L$ eine Bestimmung der Schallgeschwindigkeit aus der Lage der Resonanzfrequenzen erlaubt:

$$
k_{n} \cdot L=n \cdot \pi \Longleftrightarrow f_{n}=n \cdot \frac{c_{S}}{2 \cdot L}, \quad n \in \mathbb{N}
$$

Diese Gleichung kann für kleine Dämpfungen um eine Zellresonanzfrequenz entwickelt werden und man erhält einen Zusammenhang zwischen der Halbwertsbreite $\Delta f$ der Resonanzkurve und der Dämpfung des gesamten resonierenden Systems:

$$
\alpha=\frac{\pi}{c_{S}} \cdot \Delta f
$$

In der Praxis sind die oben getroffenen Annahmen schallharter und unendlich ausgedehnter Wandler allerdings nicht erfüllt, so dass einige weitere Faktoren mit einberechnet werden müssen:

Durch die endliche Ausdehnung der Schallwandler kommt es am Rand, insbesondere bei großen Wellenlängen, also tiefen Frequenzen, zu Beugungsverlusten. In den beiden benutzten Resonatoren sind diese Verluste zwar durch eine spezielle fokussierende Geometrie minimiert, allerdings werden die Beugungsverluste dennoch zu tiefen Frequenzen sehr groß. Labhardt berechnet in [27] explizit die Beugungsverluste für einen Resonator mit einem Paar identischer planarer Wandlerquarze:

$$
\alpha_{\text {Beugung }}=\frac{0.147}{\beta} \cdot \frac{c_{S}^{2}}{R^{3} \cdot f^{2}},
$$

wobei $\beta=Z_{F l} / Z_{\text {Wand }}$ das Impedanzverhältnis von Flüssigkeit zur Berandung bedeutet und $R$ den Wandlerradius. Für Resonatoren mit fokussierender Geometrie sind solche Zusammenhänge im Allgemeinen nicht analytisch berechenbar. Weiterhin ist wie oben angesprochen die Grenzfläche zwischen Flüssigkeit und Wandler kein schallharter Übergang. Genaue Überlegungen zeigen, dass der Reflexionsfaktor kleiner als eins und frequenzabhängig ist. Daher kann die Schallwelle in die Schallwandler eindringen und erfährt auch dort eine nicht ohne weiteres berechenbare Dämpfung. Da also die Gesamtdämpfung des resonierenden Systems wesentlich höher als die interessierende Dämpfung der Flüssigkeit ist, bedient man sich der Hilfe einer Referenzmessung, um aus der messbaren Gesamtdämpfung die Flüssigkeitsdämpfung zu bestimmen. Unter der Annahme, dass die verschiede- 
nen Dämpfungsanteile additiv sind und die apparative Dämpfung für Flüssigkeiten mit gleicher akustischer Impedanz gleich ist, gilt:

$$
\begin{aligned}
\alpha_{F l} & =\alpha_{G e s, F l}-\alpha_{a p p, F l} \\
& =\alpha_{G e s, F l}-\left(\alpha_{G e s, R e f}-\alpha_{R e f}\right)
\end{aligned}
$$

Da für die Berechnung nach dieser Gleichung die Dämpfung der Referenzflüssigkeit gut bekannt sein muss und sich weiterhin die akustische Impedanz der Referenzflüssigkeit möglichst wenig von der der Messflüssigkeit unterscheiden sollte, wurde als Referenz für alle Messungen dieser Arbeit Wasser gewählt. Wegen der geringen Konzentrationen der untersuchten Lösungen weichen sowohl die Dichte der Lösungen als auch ihre Schallgeschwindigkeit nie um mehr als $4 \%$ von den Wasserwerten ab, und somit ist auch die Bedingung einer geringen Differenz in der akustischen Impedanz erfüllt.

Aus dem nicht schallharten Übergang zwischen Flüssigkeit und Wandler ergeben sich zwei weitere Konsequenzen, die im Messablauf von Bedeutung sind:

Zum einen sind die Resonanzfrequenzen nicht mehr äquidistant, wie in Gl. (2.32) angegeben, sondern der Abstand zwischen zwei Resonanzfrequenzen $f_{j}$ und $f_{j+1}$ muss mit einem Korrekturterm nach [27] berechnet werden:

$$
\begin{aligned}
& f_{j}-f_{j-1}=\frac{c_{S}}{2 \cdot \pi \cdot L} \cdot \arccos \left(\frac{\left(g_{j}^{2}-1\right) \cdot\left(1-g_{j-1}^{2}\right)-4 \cdot g_{j} \cdot g_{j-1}}{\left(g_{j}^{2}+1\right) \cdot\left(g_{j-1}^{2}+1\right)}\right) \\
& g_{j}=\frac{\rho \cdot c_{S}}{\rho_{W} \cdot c_{W} \cdot \tan \left(\pi \cdot \frac{f_{j}}{f_{W}}\right)}
\end{aligned}
$$

Hierbei sind $\rho_{W}, c_{W}$ und $f_{W}$ die Dichte, die Schallgeschwindigkeit $\left(c_{W} \approx 5000 \mathrm{~m} / \mathrm{s}\right)$ und die Grundresonanzfrequenz des Schallwandlers, wobei letztere von der Dicke $d$ des Wandlers abhängt:

$$
f_{W}=\frac{c_{W}}{2 \cdot d}
$$

Zum anderen ist die Länge $L$, die in den Gleichungen (2.30) - (2.32) und (2.36) verwendet wird, nicht nur der Abstand zwischen den beiden Wandlern, sondern zusätzlich noch die (frequenzabhängige) Eindringtiefe der Schallwelle. Diese kann zwar nach [28] theoretisch berechnet werden, aber als praktikablere Lösung wird hier ebenfalls eine Referenzmessung gewählt, zumal auch der mechanische Abstand zwischen den Wandlern durch Temperaturunterschiede, Umbauten etc. nicht sicher bekannt ist. In der vorliegenden Arbeit wurde die Zelllängenbestimmung aus der gleichen Messung an Wasser als Referenz bestimmt, aus der auch die apparative Dämpfung bestimmt wurde, da auch die Schallgeschwindigkeit und die Dichte von Wasser sehr gut bekannt sind [22].

In Abbildung 11 ist das Blockschaltbild des Resonatormessplatzes gegeben und in Abbildung 12 eine schematische Darstellung des benutzten 1-MHz-Spiegelresonators [29]. Weiterhin sind in Tabelle 2.3 die Basisparameter der beiden benutzten Resonatoren aufgelistet. Sie unterscheiden sich hauptsächlich durch zwei Merkmale: Zum einen sind die geometrischen Abmessungen so gewählt, dass für die unterschiedlichen Grundresonanzfrequenzen 
der eingebauten Wandler jeweils ein möglichst großer Messbereich zugänglich wird, und zum anderen ist die fokussierende Wirkung konkaver Schallwandler auf unterschiedliche Arten realisiert. Im 8-MHz-Resonator sind die Wandler unter leichtem Druck eingespannt, so dass sie in der Mitte etwas nach außen gedrückt werden. Im 1MHz-Spiegel-Resonator hingegen wird die fokussierende Wirkung durch zwei konkave Glasspiegel erreicht, die sich jeweils vor den Schallwandlern befinden. In beiden Fällen wird so der Vorteil einer fokussierenden Geometrie ausgenutzt, ohne dass die Wandler selber kostenintensiv konkav geschliffen werden müssen.

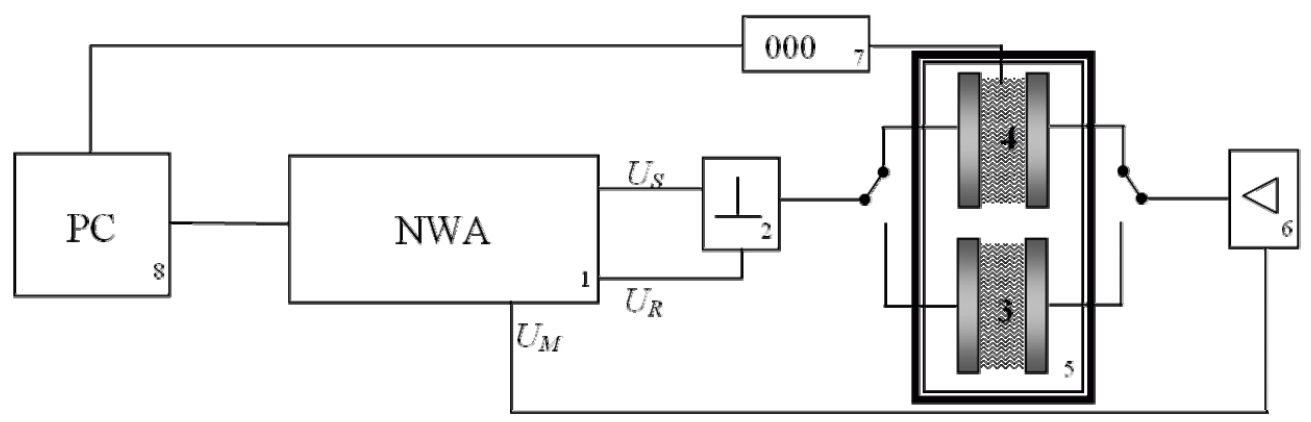

Abbildung 11: Blockschaltbild der Resonator-Messapparatur: Das im (1) Netzwerkanalysator (NWA) generierte Signal $U_{S}$ geht in einen (2) Leistungsteiler, der ein Referenzsignal an den NWA zurückgibt und das Signal an den (3) 1-MHz- oder den (4) 8-MHz-Resonator weitergibt (manuell umschaltbar), die in einer (5) gemeinsamen Thermostatierhülle stehen. Das Signal wird nach Durchlaufen der Messflüssigkeit über einen (6) Vorverstärker zurück an den NWA gegeben. Dieser sendet das Signalverhältnis $U_{M} / U_{R}$ an den (8) PC, der auch den (7) Thermofühler ausliest.

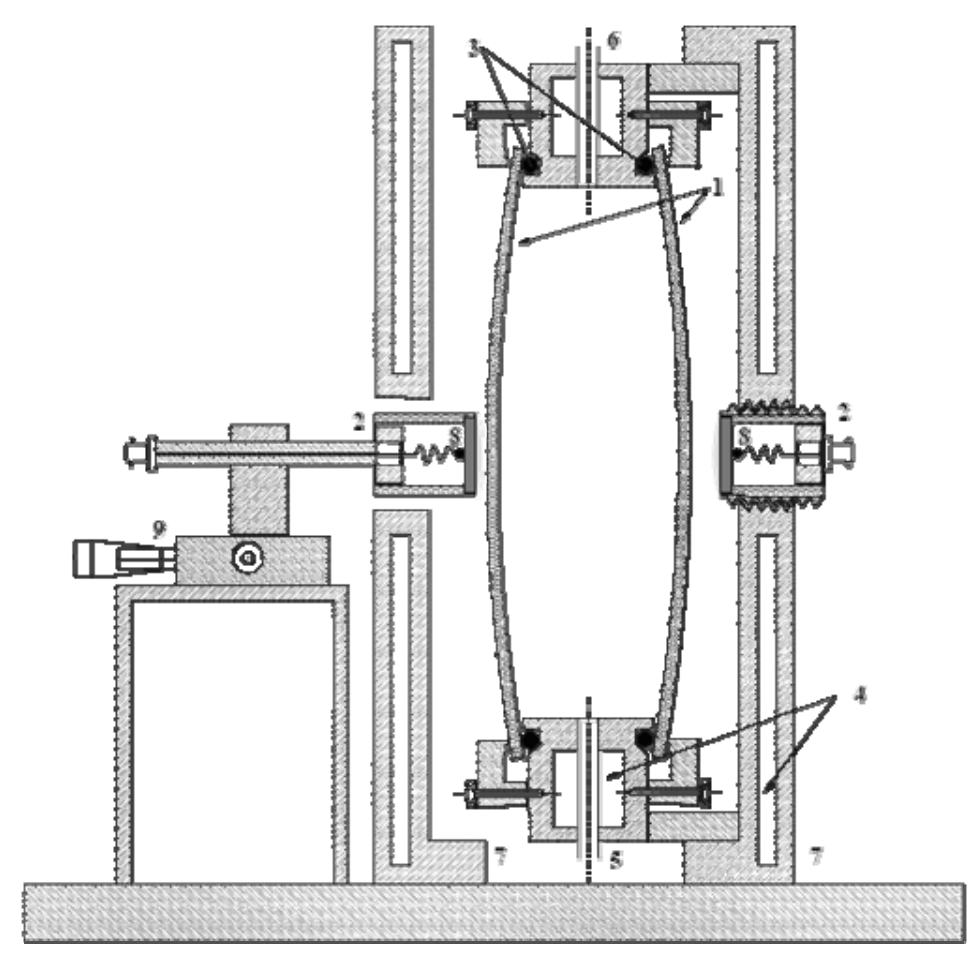

Abbildung 12: Schematische Darstellung des 1-MHz-Spiegel-Resonators: (1) Reflektor; (2) Wandlerfassung; (3) O-Ring; (4) Thermostatierkanal; (5) Befüllkanal; (6) Belüftungskanal; (7) Grundplatte; (8) Wandler; (9) Verfahreinheit 


\begin{tabular}{l|r|r|r|r|r|r}
\hline \hline Resonator & \multicolumn{1}{|c|}{$\begin{array}{c}f_{W} \\
{[\mathrm{MHz}]}\end{array}$} & $\begin{array}{c}R_{W} \\
{[\mathrm{~mm}]}\end{array}$ & \multicolumn{1}{c|}{$\begin{array}{c}R_{\text {Zelle }} \\
{[\mathrm{mm}]}\end{array}$} & $\begin{array}{c}L \\
{[\mathrm{~mm}]}\end{array}$ & \multicolumn{1}{c|}{$\begin{array}{c}V \\
{[\mathrm{~mL}]}\end{array}$} & $\begin{array}{c}\text { Messbereich } \\
{[\mathrm{MHz}]}\end{array}$ \\
\hline \hline $1-\mathrm{MHz}$ & 1.1 & 40 & 35 & 19 & $\sim 35$ & $0.1-1.9$ \\
\hline $8-\mathrm{MHz}$ & 8 & 10 & 8.5 & 6 & $\sim 5$ & $0.3-23$ \\
\hline \hline
\end{tabular}

Tabelle 2.3: Basisparameter der benutzten Resonatoren: Wandlergrundresonanzfrequenz $f_{W}$, Wandlerradius $R_{W}$, Zellradius $R_{\text {Zelle, }}$, ungefähre Zelllänge $L$, Zellenvolumen $V$ und theoretischer maximaler Messbereich.

In der Praxis treten bei den Resonatormessungen außer der von der geometrischen Länge abweichenden effektiven Zelllänge und den korrigierten Resonanzabständen gemäß Gl. (2.36) noch einige weitere zu beachtende Besonderheiten auf:

Zum einen weist das Übertragungsspektrum jedes Resonators wegen seiner endlichen lateralen Ausdehnung außer den Hauptresonanzen noch radiale Nebenmoden zu jeder Resonanz auf, die sich jeweils an die hochfrequente Seite der Resonanz anschließen. Die Abstände zwischen der Hauptmode und der ersten Nebenmode und zwischen zwei aufeinanderfolgenden Nebenmoden sind in Resonatoren mit fokussierender Geometrie bei hohen Frequenzen nicht von der Frequenz abhängig [27]. Da jedoch bei hohen Frequenzen die Halbwertsbreiten der Nebenmoden genau wie die der Hauptmoden aufgrund der größer werdenden Hintergrundsdämpfung ansteigen, überlappen dort die Haupt- und die Nebenmoden, was bei der Anpassrechnung (siehe unten) berücksichtigt werden muss.

Zum anderen sind die Wandler selbst schwingungsfähige Systeme mit einer Resonanzfrequenz gemäß Gl. (2.37). Das gesamte Übertragungsspektrum ist daher durch eine Kopplung zwischen der sehr breiten Resonanzkurve der Wandlerresonanz und den vergleichsweise schmalen Resonanzkurven der einzelnen Flüssigkeitsresonanzen gegeben. Dies führt im Bereich der jeweiligen Wandlerresonanz (1 MHz beim 1-MHz-Spiegel-Resonator und 8 $\mathrm{MHz}$ beim 8-MHz-Resonator) zu nichtlinearen Verzerrungen der Flüssigkeitsresonanzen, so dass in dem jeweiligen Bereich keine sinnvollen Messungen möglich sind. Zudem weisen alle benutzten Schallwandler Scherresonanzen bei $f_{\text {Scher }}=n \cdot 0.627 \cdot f_{W}$ auf, bei denen ebenfalls keine Messpunkte aufgenommen werden sollten.

Weiterhin tritt bei den Resonatoren wie schon bei den Ultraschalltransmissionsapparaturen ein elektrisches Übersprechen auf. Auch dieses muss bei der Anpassrechnung der Übertragungsfunktion beachtet werden.

Ein konkreter Messablauf sieht so aus, dass zunächst die Referenzmessung an Wasser vorgenommen wird. Dazu werden die einzelnen Resonanzkurven aufgenommen und mit Gl. (2.36) wird aus den Resonanzabständen die effektive Zelllänge $L$ bestimmt um damit die Anpassrechnung der Übertragungsfunktion

$$
T(f)=\left(\sum_{k=1}^{N} \frac{A_{k}}{\sinh \left(\gamma_{k} \cdot L\right)}+U \cdot e^{i \Phi_{U}}\right) \cdot e^{i \Phi_{0}} \cdot E(f)
$$

an jede einzelne aufgenommene Resonanzkurve vornehmen zu können. Hier ist $N$ die Anzahl der insgesamt aufgenommenen Moden (Hauptmode und Nebenmoden), $\gamma_{k}=\alpha_{k}+i \cdot 2 \pi \cdot f_{k} / c_{S}, U$ die Amplitude und $\Phi_{U}$ die Phase des elektrischen Übersprechens, $\Phi_{0}$ eine globale Phase und $E(f)$ ist eine Funktion, die die erwähnte Frequenzabhängigkeit der Schallwandler beschreibt. Der somit ermittelte Absorptionskoeffizient entspricht dann $\alpha_{G e s, R e f}$ in Gl. (2.35). Anschließend kann die Messung an der Messsubstanz vorgenommen werden, indem wiederum die ein- 
zelnen Resonanzkurven aufgenommen werden, aus deren Lage nach Gl. (2.36) die Schallgeschwindigkeit ermittelt und dann jede einzelne Resonanzkurve nach Gl. (2.38) angepasst wird. Die hieraus resultierenden Werte von $\alpha_{G e s, F l}$ werden dann mit Gl. (2.35) in die erwünschten $\alpha_{F l}$-Werte umgerechnet.

Sowohl bei der der Referenz- als auch bei der Substanzmessung muss die jeweilige Flüssigkeit vor der Messung sorgfältig entgast und langsam und blasenfrei in die Messzelle gefüllt werden, da kleine Luftblasen oder schlecht benetzte Wandler die Messungen erheblich verfälschen könnten. Zudem wird vor jeder Messung die Temperaturstabilität kontrolliert, indem eine ausgewählte Resonanzkurve solange immer wieder aufgenommen wird, bis die Halbwertsbreite und die Mittenfrequenz der Resonanz hinreichend konstant sind. Die Thermostatierung der Resonatoren erfolgt wie bei den Ultraschalltransmissionsapparaturen über einen inneren Thermostatierkreislauf, der die beiden Messzellen auf die gewünschte Messtemperatur bringt, und einen separaten äußeren Kreislauf, der die in Abbildung 11 dargestellte Thermostatierhülle temperiert. Wie beim Ultraschalltransmissionsverfahren wird damit auch bei den Resonatormessungen eine Temperaturkonstanz und -genauigkeit von $\Delta T=0.02 \mathrm{~K}$ erreicht.

Der Messfehler ist beim Resonatorverfahren von vielen verschiedenen Faktoren wie der Frequenz, der Dämpfung der Substanz und der Differenz zwischen Substanz- und Referenzimpedanz abhängig. Bei den in dieser Arbeit vorgenommenen Messungen kann der Fehler allerdings als $\Delta \alpha / \alpha<5 \%$ abgeschätzt werden; lediglich bei den Messungen an Lösungen mit sehr geringen Dämpfungen $\left(\alpha / f^{2}<5 \cdot 10^{-14} \mathrm{~s}^{2} / \mathrm{m}\right)$ muss von einem größeren Messfehler $\Delta \alpha / \alpha \approx 15 \%$ ausgegangen werden. 



\section{Alkylglykoside}

Alkylglykoside bestehen, wie der Name bereits verrät, aus einer Alkylkette, also einer Kohlenwasserstoffkette mit der Summenformel $\mathrm{C}_{\mathrm{n}} \mathrm{H}_{2 \mathrm{n}+1}$ und der Strukturformel $-\left(\mathrm{CH}_{2}\right)_{\mathrm{n}-1}-\mathrm{CH}_{3}$, und einem Glykosid-Teil, also einem über ein Sauerstoffatom an die Alkylkette gebundenen Zucker. Als Zucker bezeichnet man allgemein Mono- und Oligosaccharide mit bis zu sechs Saccharideinheiten. In der vorliegenden Arbeit wurden Alkylketten mit der Kettenlänge $6 \leq \mathrm{n} \leq 12$ benutzt und als Glykoside wurden Maltoside, Glukoside und Galaktoside benutzt. Wie in der Literatur zumeist üblich wird für die Substanzen die Abkürzung $\alpha-\beta-\mathrm{C}_{\mathrm{i}} \mathrm{G}_{\mathrm{j}}$ bzw. $\mathrm{C}_{\mathrm{i}} \mathrm{Gal}$ benutzt, wobei i die Alkylkettenlänge angibt und $\mathrm{j}$ die Anzahl der Glukose-Einheiten in der Kopfgruppe ( $\mathrm{j}=1$ für Glukose, $\mathrm{j}=2$ für Maltose) und das vorgestellte , $\alpha$ ' oder , $\beta$ ' den Bindungstyp der Alkylkette an die Kopfgruppe (axial bzw. äquatorial, siehe Kapitel 3.1).

Tenside finden in den unterschiedlichsten Bereichen des täglichen Lebens, der Industrie und der Wissenschaft Anwendung - so werden sie unter anderem in Shampoos, Duschgels, Seifen, Waschmitteln, Spülmitteln, Weichmachern, Klebstoffen, Farben, Ski-Wachs, Herbiziden und Insektiziden eingesetzt, aber auch zur Denaturierung von Proteinen, zur Solubilisierung von Membranproteinen oder als Fließverbesserer in Löschwasser [3]. Bei der Weiterentwicklung und Verbesserung all dieser Produkte steht natürlich neben der Suche nach preisgünstigen Rohstoffen seit einigen Jahrzehnten auch die Umweltverträglichkeit im Vordergrund. Die Tatsache, dass die hydrophile Kopfgruppe der Alkylglykoside aus einem nichtgiftigen, biologisch abbaubaren, nachwachsenden und relativ preisgünstigen Rohstoff (Zucker) besteht, macht diese Substanzklasse daher schon seit geraumer Zeit hochinteressant für den Einsatz in industriellen Produkten. Obwohl Alkylglykoside von Emil Fischer bereits 1893 zum ersten Mal synthetisch hergestellt wurden, gelang es erst in den 1990er Jahren, die nach Fischer benannte Fischer-Glykosylierung (einer sauer katalysierten Reaktion von Glukose mit Alkohol) so umzusetzen, dass sie für automatisierte industrielle Großproduktionen interessant wurde [30].

Für industrielle Anwendungen spielen dabei polydisperse Mischungen, also Mischungen aus Alkylglykosiden mit unterschiedlichen Kopfgruppen und unterschiedlichen Alkylkettenlängen, wegen ihrer wesentlich günstigeren Herstellung eine größere Rolle als die in dieser Arbeit untersuchten monodispersen Systeme, deren Verständnis aber natürlich als Grundlage für das Verständnis polydisperser Mischungen unerlässlich ist

Da in dieser Arbeit Alkylglykoside mit drei verschiedenen Glykosidköpfen untersucht wurden, wird in Kapitel 3.1 zunächst ein Überblick über die Substanzklasse der Glykoside und insbesondere ihre Nomenklatur gegeben und in Kapitel 3.2 werden die bisher in der Literatur gewonnenen Ergebnisse zu Konformationsänderungen von Glykosiden zusammengefasst. In Kapitel 3.3 und 3.4 werden die speziellen Mechanismen der Mizellkinetik- und geometrie im Allgemeinen vorgestellt, bevor in Kapitel 3.5 Erkenntnisse zu diesen Themen aus der Literatur zusammengefasst werden. Kapitel 3.6 beschäftigt sich mit der Substanzklasse der Cyclodextrine und speziell mit der in dieser Arbeit untersuchten Komplexbildung zwischen Cyclodextrinen und Tensiden. In Kapitel 3.7 schließlich werden die in dieser Arbeit untersuchten Substanzen mit einigen Basisparametern aufgelistet und das Ansetzen der Messlösungen beschrieben. 


\subsection{Glykoside}

„Glykoside sind Äther der Kohlenhydrate (Mono- oder Oligosaccharide), wobei der mit dem Zucker verbundene Rest (das sogenannte Aglykon) fast immer über das Sauerstoffatom am anomeren C-Atom an das Kohlenhydrat gebunden ist. Je nach dem Kohlenhydratbaustein spricht man von Glukosid, Mannosid, Galaktosid usw.; die Ringgröße des Halbacetalrings wird durch die Bezeichnung -pyranosid bzw. -furanosid angegeben." [31]

1844 prägte K. Schmidt den Begriff ,Kohlenhydrate', da viele Substanzen dieser Klasse die Summenformel $\mathrm{C}_{\mathrm{n}}\left(\mathrm{H}_{2} \mathrm{O}\right)_{\mathrm{m}}$ (,Hydrate der Kohle') aufweisen. Allerdings wird mittlerweile im Allgemeinen der Begriff ,Saccharid' bevorzugt, da eben nicht alle Substanzen dieser Klasse obige Summenformel aufweisen, bzw. einige Substanzen dieser Summenformel nicht zur Substanzklasse der Kohlenhydrate gehören. Allgemein werden als Saccharide alle Hydroxyaldehyde, Hydroxyketone sowie davon abgeleitete Verbindungen definiert [32].

a-D-Glukopyranose

Abbildung 13: Schematische Darstellung von sechs Glukose-Isomeren sowie $\beta$-DGalaktopyranose und $\beta$-D-Maltose in der Konformationsdarstellung. Die Pyranosen sind in Sesselform dargestellt. Die Darstellungen sind so gewählt, dass die Nummerierung der Kohlenstoffatome vom jeweils am weitesten rechts liegenden C-Atom anfangend im Uhrzeigersinn erfolgt. 
Zum Verständnis der Namensgebungen der betrachteten Substanzen soll hier am Beispiel der $\beta$-DGlukopyranose eine Erklärung der Nomenklatur der Saccharide gegeben werden.

Der vorgestellte griechische Buchstabe $\alpha$ oder $\beta$ unterscheidet zwischen den beiden Diastereomeren, bei denen die Hydroxylgruppe axial $(\alpha)$ bzw. äquatorial $(\beta)$ an das $\mathrm{C}_{1}$-Atom ${ }^{4}$ des Glukoserings gebunden ist (siehe Abbildung 13), ein vorgestelltes L oder D unterscheidet zwischen den beiden Enantiomeren, bei denen die Hydroxylgruppe des am weitesten von der Carbonyl-Gruppe entfernten asymmetrisch substituierten Kohlenstoffatoms in der Fischer-Projektion nach links (L) bzw. nach rechts (D) zeigt. Weiterhin gibt der Namensteil ,pyrano' an, dass der Glukosering aus fünf Kohlenstoffatomen und einem Sauerstoffatom besteht, so dass das $\mathrm{C}_{6^{-}}$ Atom außerhalb des Ringes liegt, während ein Namensteil ,furano' auf einen Ring aus vier Kohlenstoffatomen und einem Sauerstoffatom sowie zweier Kohlenstoffatome außerhalb des Ringes hindeutet.

In Abbildung 13 sind außer den sechs Glukose-Isomeren zusätzlich noch Galaktopyranose und Maltopyranose dargestellt, da in dieser Arbeit auch Alkylglykoside mit diesen beiden Substanzen als Kopfgruppen gemessen wurden. Galaktopyranose ist ein Diastereomer der Glukose, das sich nur dadurch von jener unterscheidet, dass die Hydroxyl-Gruppe am $\mathrm{C}_{4}$-Atom axial (Galaktopyranose) statt äquatorial (Glukopyranose) vorliegt. Während die Stellung der Hydroxyl-Gruppe am $\mathrm{C}_{1}$-Atom im Allgemeinen nur zu leichten Unterschieden in den physikalischen und chemischen Eigenschaften führt und daher nur durch das Präfix $\alpha$ - oder $\beta$ - gekennzeichnet wird, unterscheiden sich die Eigenschaften der beiden $\mathrm{C}_{4}$-Diastereomere so sehr, dass sie mit verschiedenen Namen bezeichnet werden.

Als einziges Disaccharid ist in Abbildung 13 die $\beta$-D-Maltopyranose aufgeführt, die aus zwei $\alpha(1,4)$ glykosidisch gebundenen Glukopyranosen besteht, das heißt, dass die Bindung über ein Sauerstoffatom erfolgt, das axial an das $\mathrm{C}_{1}$-Atom des ersten Glukoseringes und äquatorial an das $\mathrm{C}_{4}$-Atom des zweiten Glukoseringes bindet.

\footnotetext{
${ }^{4}$ Die Kohlenstoffatome der Saccharide werden durchnummeriert, beginnend bei dem Atom, das der Aldehydbzw. Keto-Gruppe am nächsten ist.
} 


\subsection{Konformationsänderungen von Glykosiden}

Die Konformationsvielfalt und -kinetik von Glykosiden ist seit Jahrzehnten ein Gebiet von hohem Interesse in der Forschung, insbesondere natürlich an Glukose. Zum einen ist Glukose selber als Blutzucker des Menschen und als Energiequelle bei der Bildung von Adenosintriphosphat (ATP) von zentraler Bedeutung für das Verständnis der Vorgänge im Körper in Medizin, Biochemie und Biophysik. Zum anderen sind Derivative der Glukose relevant bei Erkennungsprozessen auf zellulärer Ebene, indem sie bestimmte Proteine (Lektine und Selektine) spezifisch an Zellmembranen binden können. Nicht zuletzt dienten Untersuchungen an dem enzymatischen Komplex Glukose-Hexokinase der Verifizierung und dem Verständnis des ,induced-fit'-Modells [16] molekularer Erkennung, bei dem in Erweiterung des vorher vorherrschenden Schlüssel-Schloss-Modells davon ausgegangen wird, dass Enzym und Substrat bei der Annäherung gegenseitig Konformationsänderungen induzieren und erst dadurch eine spezifische Erkennung gewährleistet ist.

Aus der sehr umfangreichen Vielfalt von Konformationsänderungen sollen hier nur die erwähnt werden, die in den Ultraschallabsorptionsspektren der untersuchten Alkylglykoside auftreten. Für weitere Konformationsänderungen, wie z.B. der Mutarotation oder der Umwandlung zwischen Sessel-, Halb-Sessel-, Twist- und Wannenkonformation, sei auf z.B. [33] oder [34] verwiesen.

Zur Veranschaulichung sind in Abbildung 14 die beiden in der Ultraschallabsorptionsspektroskopie auftretenden und detektierbaren Konformationsänderungen an einem Maltosemolekül als Beispiel schematisch dargestellt. Im linken Teil sind die beiden sogenannten glykosidischen Bindungswinkel $\varphi$ und $\psi$ gekennzeichnet, im rechten Teil der Bindungswinkel $\omega$ der exozyklischen $\mathrm{CH}_{2} \mathrm{OH}-$ Gruppe (Hydroxymethyl-Gruppe).
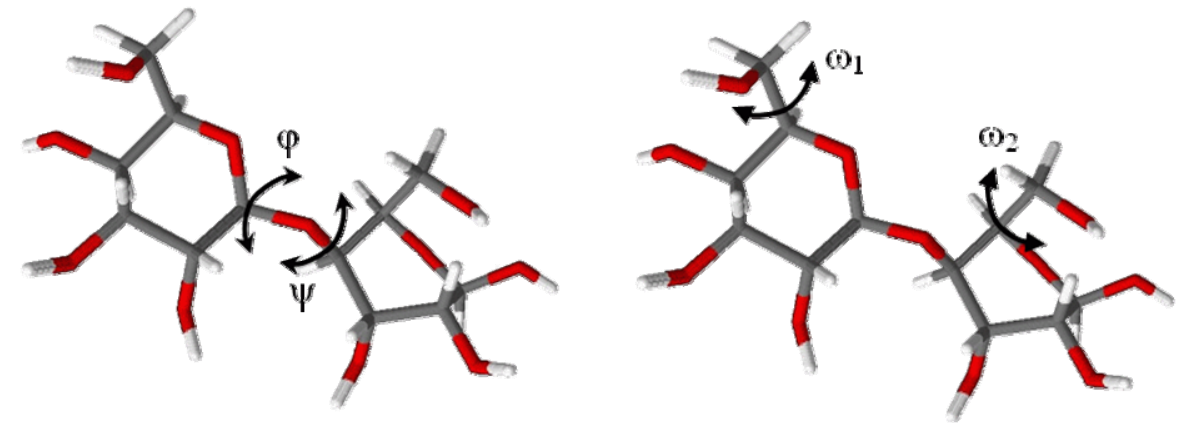

Abbildung 14: Schematische Darstellung der Konformationsänderungen von Maltose. Die Rotation um die glykosidischen Bindungswinkel $\varphi$ und $\psi$ (links) und die Rotation der exozyklischen Gruppe um den Winkel $\omega$ (rechts). 


\subsubsection{Die glykosidischen Bindungswinkel $\varphi$ und $\psi$}

In Abbildung 15 ist am Beispiel der Maltose eine Verdeutlichung der Definition der beiden glykosidischen Bindungswinkel $\varphi$ (links) und $\psi$ (rechts) und insbesondere ihrer jeweiligen Nullstellung gegeben. Der Winkel $\varphi$ ist dabei definiert als $\varphi \equiv-\Varangle\left(\mathrm{H}_{1}, \rightarrow \mathrm{C}_{1}, \mathrm{O}_{1} \rightarrow \mathrm{C}_{4}\right)$, also als der negative Winkel zwischen der Achse $\mathrm{H}_{1}, \rightarrow \mathrm{C}_{1}$, und der Achse $\mathrm{O}_{1} \rightarrow \mathrm{C}_{4}$, bzw. einfach dem Winkel zwischen $\mathrm{H}_{1}$, und $\mathrm{C}_{4}$ bei Blickrichtung $\mathrm{C}_{1}, \rightarrow \mathrm{O}_{1}$, Im oberen Teil der Abbildung ist das Maltose-Molekül mit $\varphi \approx 0^{\circ}$, der sogenannten, syn'-Konfiguration, im unteren Teil mit $\varphi \approx-60^{\circ}$, der ,gauche'-Konfiguration, dargestellt.
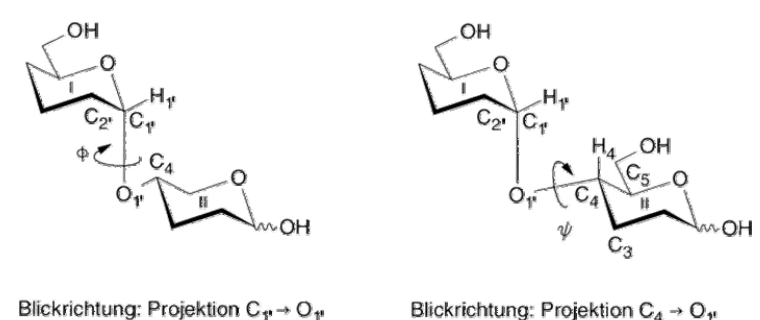

Blickrichtung: Projektion $\mathrm{C}_{4} \rightarrow \mathrm{O}_{1}$
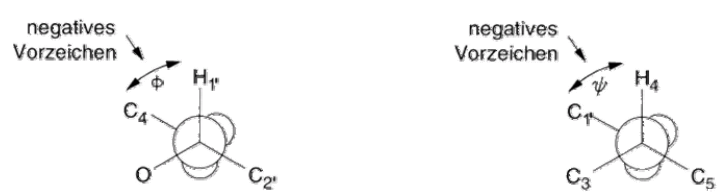

\section{Abbildung 15: Definition der glykosidischen Bindungswinkel $\varphi$ und $\psi$ am Beispiel der Maltose, ent- nommen aus [33].}

Analog gilt für die Definition von $\psi: \psi \equiv-\Varangle\left(\mathrm{H}_{4} \rightarrow \mathrm{C}_{4}, \mathrm{O}_{1}, \rightarrow \mathrm{C}_{1}\right)$; im oberen Teil der Abbildung ist dementsprechend $\psi \approx 0^{\circ}$ (, syn') und im unteren Teil $\psi \approx-60^{\circ}$ (, gauche').

Aus Molekulardynamiksimulationen existieren Konturdiagramme für Maltose, die die Konformationsenergie des Moleküls in Abhängigkeit von den beiden Winkeln $\varphi$ und $\psi$ angeben. In Abbildung 16 sind solche Konturdiagramme nach [35], [36] und [37] dargestellt. Der Verlauf der Äquipotentiallinien und auch die konkreten Konformationsenergien unterscheiden sich in den einzelnen Diagrammen zwar leicht, aber in allen Fällen wird offensichtlich, dass nur zwei stabile Winkelkombinationen $([\varphi, \psi] \approx[-60,0]$ (,syn') und $[\varphi, \psi] \approx[-20,180]($,trans')) existieren.

Hagen findet in Ultraschallabsorptionsspektren von Maltose- und anderen Disaccharid-Lösungen einen Prozess mit einer Relaxationszeit von ca. 6 ns, den er einer teilweisen Rotation um $\varphi$ und/oder $\psi$ zuordnet [38]. Eine vollständige ,syn'-,trans'-Konformationsumwandlung wird ausgeschlossen, da die einzige Molekulardynamiksimulation, die mit einer Simulationszeit von $2 \mu$ s lang genug für das mehrfache Beobachten einer solchen Umwandlung ist, jene nur viermal aufweist [39]. Daraus wäre eine Relaxationszeit in der Größenordnung von $\tau \approx 500$ ns für diesen Prozess zu erwarten. Eben jene Simulation deutet aber weiterhin auf die Existenz vier lokaler Minima innerhalb des globalen Minimums der ,syn'-Konformation hin. Eine Zuordnung des in der Ultraschallabsorptionsspektroskopie sichtbaren Prozesses bei etwa $6 \mathrm{~ns}$ zu Umwandlungen zwischen diesen Minima erscheint daher plausibler. 

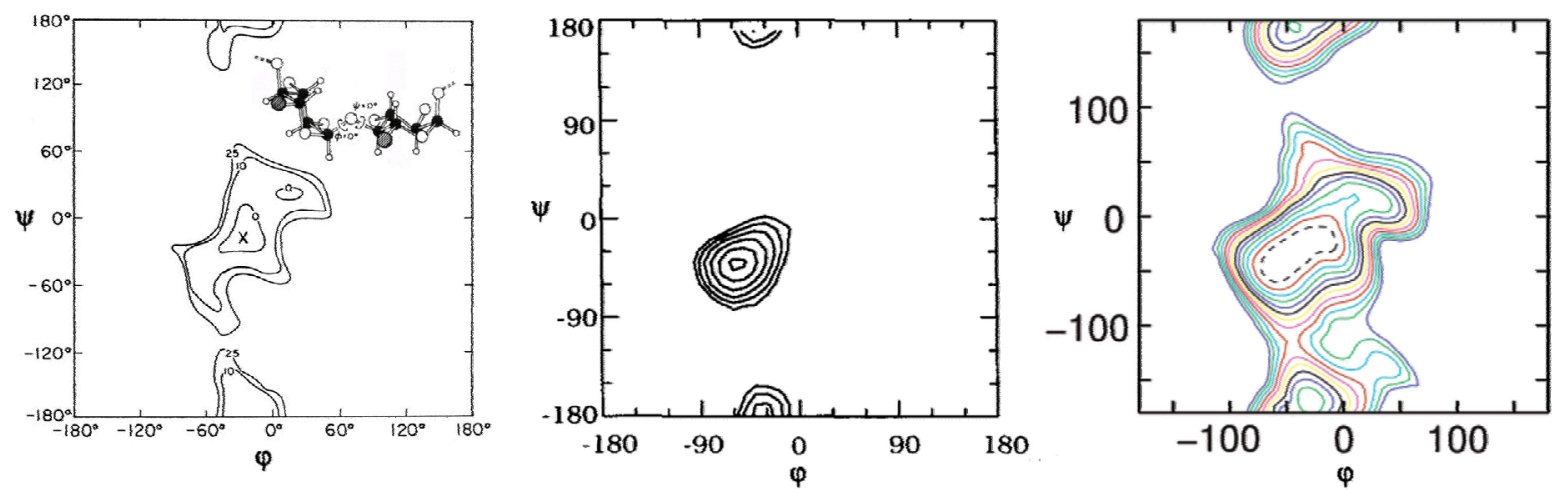

Abbildung 16: Konturdiagramme der glykosidischen Bindungswinkel $\varphi$ und $\psi$. Im linken Diagramm (entnommen aus [35]) stehen die jeweiligen Konformationsenergien in $\mathrm{kcal} / \mathrm{mol}$ an den Äquipotentiallinien, im mittleren Diagramm (entnommen aus [36]) entsprechen die Konturlinien von außen nach innen etwa $2.5,2,1.5,1,0.5,0.1 \mathrm{kcal} / \mathrm{mol}$ und im rechten Diagramm (entnommen aus [37]) $1 \mathrm{kcal} / \mathrm{mol}$ für die gestrichelte Linie und jeweils $1 \mathrm{kcal} / \mathrm{mol}$ mehr für jede weitere Linie.

Während sich die verschiedenen Simulationen in der Literatur in den Details unterscheiden, kommen hingegen alle Untersuchungen, in denen verschiedene Disaccharide betrachtet werden, zu der Erkenntnis, dass die Konformationsenergien vor allem von der Geometrie des jeweiligen Moleküls abhängig sind, so dass für unterschiedliche Disaccharide auch deutlich unterschiedliche Konturdiagramme resultieren [40] - [42].

Interessant wird daher bei den Messungen an Alkylglykosid-Lösungen zu beobachten sein, ob überhaupt ein solcher Prozess in den Ultraschallabsorptionsspektren auftritt, wenn statt eines zweiten Saccharids wie bei den Disacchariden eine Alkylkette an der glykosidischen Bindung gebunden ist. Falls dieser Prozess auch bei den Alkylglykosiden auftritt, stellt sich weiterhin die Frage, wie die veränderte geometrische Situation den Prozess beeinflusst. 


\subsubsection{Der Bindungswinkel $\omega$ der exozyklischen Hydroxymethylgruppe}

Der Bindungswinkel $\omega$ der exozyklischen Hydroxymethylgruppe ist definiert als der Winkel zwischen der Achse $\mathrm{O}_{5} \rightarrow \mathrm{C}_{5}$ und der Achse $\mathrm{C}_{6} \rightarrow \mathrm{O}_{6}$, also dem Winkel zwischen $\mathrm{O}_{5}$ und $\mathrm{O}_{6}$ bei Blickrichtung $\mathrm{C}_{5} \rightarrow \mathrm{C}_{6}$ : $\omega \equiv \Varangle\left(\mathrm{O}_{5} \rightarrow \mathrm{C}_{5}, \mathrm{C}_{6} \rightarrow \mathrm{O}_{6}\right)$. In Abbildung 17 ist die Definition verdeutlicht und drei mögliche Konformationen von Methyl- $\alpha$-D-Glukopyranosid sind schematisch dargestellt. Dabei sind bereits die in der Literatur standardisierten Bezeichnungen gt $\left(\omega \approx 60^{\circ}\right)$, $\operatorname{tg}\left(\omega \approx 180^{\circ}\right)$ und gg $\left(\omega \approx 300^{\circ}\right)$ für die drei energetisch stabilen Konformationen verwendet, wobei jeweils der erste Buchstabe die Stellung (,gauche' oder ,trans') des $\mathrm{O}_{6}$-Atoms zum $\mathrm{O}_{5}$-Atom und der zweite die Stellung des $\mathrm{O}_{6}$-Atoms zum $\mathrm{C}_{4}$-Atom angibt.

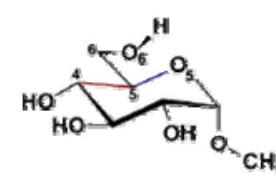

gt $\left.(\omega) \sim 60^{\circ}\right)$<smiles>CC1C2CC(C)(C(O)C2=O)C1C</smiles>

gt

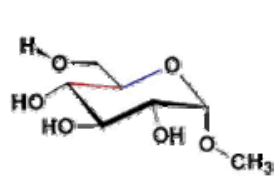

$\left.\operatorname{tg}(0) \sim 180^{\circ}\right)$

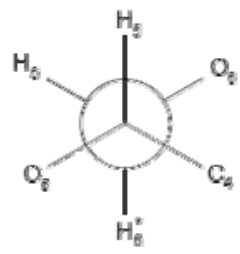

$\operatorname{tg}$

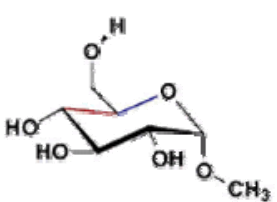

$g g\left(\omega-300^{\circ}\right)$

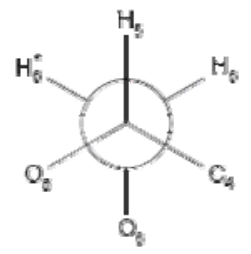

$g g$

\section{Abbildung 17: Definition des $\omega$-Winkels und Darstellung dreier möglicher Konformationen von Methyl-a-D-Glukopyranosid, entnommen aus [43].}

Bereits an obiger Abbildung wird schnell ersichtlich, dass von den drei relevanten Konformationen gt, tg und gg die tg-Stellung wegen sterischer Behinderungen zwischen dem $\mathrm{O}_{6}$ - und dem $\mathrm{C}_{4}$-Atom sehr ungünstig ist. Diverse Molekulardynamiksimulationen bestätigen dies. Wesentlich schnellere Übergangszeiten zwischen den einzelnen $\omega$-Konformationen als im Fall der glykosidischen Bindungswinkel $\varphi$ und $\psi$ ermöglichen es, für die $\omega$-Rotation trotz zeitlich begrenzter Simulationszeiten eine sinnvolle Statistik zu betreiben. Zahlreiche Ergebnisse aus Simulationen [36] - [39], [42] - [44], quantenmechanischen Berechnungen [43] und experimentellen Arbeiten wie ${ }^{1}$ H-NMR Spektroskopie [36], [50] oder Neutronenstreuung [44] ergeben (mit nur leichten Abweichungen) eine Rotamerverteilung von (gt):(tg):(gg) $\approx(60 \pm 5):(5 \pm 5):(40 \pm 5)^{5}$ und mittlere Lebensdauern des gt-/gg-Zustandes von $\approx 1 \mathrm{~ns}[42]$ bis $\approx 5 \mathrm{~ns}[43]$.

In der Ultraschallabsorptionsspektroskopie werden in allen Lösungen von Mono- und Disacchariden mit einer exozyklischen Gruppe molekulare Prozesse mit einer Relaxationszeit von ca. 1.5 \pm 0.5 ns gefunden, die der Umwandlung gt $\rightleftharpoons$ gg zugeordnet werden [45] - [48]. Auch in $\alpha$-Cyclodextrin-Lösungen (siehe Kapitel 3.6) tritt dieser Prozess auf [49].

\footnotetext{
${ }^{5}$ Dass die Summe der Populationen von 100 abweicht, liegt an der Mittelung über verschiedene Ergebnisse aus der Literatur.
} 
Interessant ist in diesem Zusammenhang, inwiefern sich die Rotamerverteilung durch Änderungen der Molekülgeometrie ändert. So ist z.B. sofort offensichtlich, dass die Stellung der OH-Gruppe am $\mathrm{C}_{4}$-Atom einen immensen Einfluss auf die Population der tg-Konformation der exozyklischen Gruppe hat. Dementsprechend weisen ${ }^{1}$ H-NMR-Messungen auch einen signifikanten Unterschied zwischen den Rotamer-Populationen von Galaktose und Glukose auf [50]. Dieser Unterschied macht sich auch in den Ultraschallabsorptionsspektren von Galaktoseund Glukose-Lösungen bemerkbar; der Prozess, der der Rotation der exozyklischen Gruppe zugeordnet wird, tritt in Galaktose-Lösungen nicht auf [46]. Erstaunlicher ist hingegen, dass auch in den Rotamer-Populationen zwischen Molekülen, die sich an weiter von der exozyklischen Gruppe entfernten Stellen unterscheiden, deutliche Unterschiede auftreten (siehe Abbildung 18). Eine breite Übersicht über die Rotamer-Verteilungen verschiedener Kohlenhydrate und stereochemische Begründungen für die Unterschiede finden sich z.B. in [51]. In den Ergebnissen dieser Arbeit wird interessant sein, inwiefern zum einen die Anwesenheit einer Alkylgruppe am $\mathrm{C}_{1^{-}}$ Atom und zum anderen die Anwesenheit direkter Nachbarn in einer Mizelle die Rotation der exozyklischen Gruppe beeinflusst.

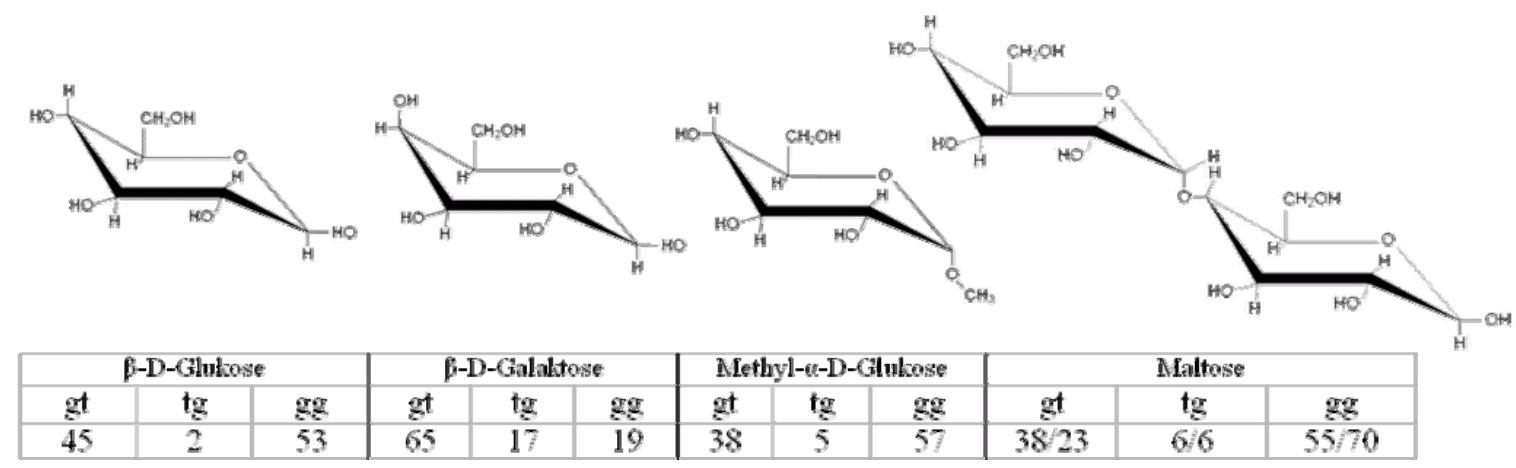

\begin{abstract}
Abbildung 18: Zusammenhang zwischen der Molekülgeometrie und der Rotamer-Population der exozyklischen Gruppe. Während der Unterschied der Rotamer-Populationen zwischen der Glukose (ganz links, Daten aus [50]) und der Galaktose (2. v. links, [51]) durch die unterschiedliche Stellung der $\mathrm{OH}$-Gruppe am $\mathrm{C}_{4}$-Atom evident ist, beeinflussen offensichtlich auch weiter entfernte Gruppen, wie z.B. eine Methyl-Gruppe (Methyl- $\alpha$-D-Glukopyranosid, 2. v. rechts, [43]) oder ein zweiter Glukose-Ring (Maltose, ganz rechts, [36]) die Rotamer-Population. Der jeweils erste Wert bei der Maltose gilt für den nichtreduzierenden Glukose-Ring (den linken in der Abbildung), der zweite für den reduzierenden (den rechten); alle Werte in \%.
\end{abstract}

Weiterhin sei angemerkt, dass aktuelle Berechnungen mithilfe der Dichtefunktionaltheorie (DFT, [52]) eine Abhängigkeit der $\varphi-/ \psi$-Konformationsverteilung von der Stellung der exozyklischen Gruppe vorhersagen [53]. Die Frage nach der experimentellen Verifizierbarkeit solcher Berechnungen und nach den Konsequenzen der Molekülgeometrie für die Konformationskinetik von (Alkyl-)Glykosiden sind daher auch aktuell noch relevant für die Stereochemie und andere Gebiete. 


\subsection{Mizellkinetik}

Die spezielle Kinetik von Mizellsystemen ist bereits seit den 1970er Jahren ein Schwerpunkt in der experimentellen und theoretischen Forschung an Tensiden. Prinzipiell unterteilt man Tenside anhand der Ladung ihrer Kopfgruppen in nichtionische, anionische, kationische und zwitterionische Tenside. Da in dieser Arbeit ausschließlich nichtionische Tenside untersucht wurden, wird im Folgenden nicht auf die teilweise vorhandenen Besonderheiten ionischer Kopfgruppen (z.B. Dissoziation von Gegenionen, Abstoßung der Kopfgruppen etc. [54]-[55]) eingegangen.

Die Frage, unter welchen Umständen (Tensidkonzentration, Temperatur, Druck, etc.) sich Tensidmoleküle in wässriger Lösung zu Mizellen zusammenschließen, beruht hauptsächlich auf zwei gegenläufigen entropischen Überlegungen: Zum einen ist die Mischungsentropie des Gesamtsystems natürlich dann maximal, wenn die Tensidmoleküle möglichst homogen und gleichmäßig in der Lösung verteilt sind, während eine lokale Häufung von Tensidmolekülen (eine Mizelle) entropisch ungünstig ist. Zum anderen können sich zwar die TensidKopfgruppen wegen ihres hydrophilen Charakters gut in die Wasserstoffbrückenbindungen des umgebenden Wassers einbinden, die hydrophoben Alkylketten aber nicht, so dass die Dynamik der umgebenden Wassermoleküle an den Alkylketten gestört wird [56]. Die Wassermoleküle können ihre Wasserstoffbrückenbindungen nur noch in einem gewissen Bruchteil ihrer Umgebung eingehen, während ein Teil des möglichen $4 \pi$-Raumwinkels ,verboten' ist: Je mehr Alkylketten ,solubilisiert' werden müssen, desto geringer wird die Bewegungs-Entropie des Wassers.

Die konkrete Form dieser beiden gegenläufigen Entropie-Terme hängt dabei zum einen von den äußeren Parametern wie Druck, Temperatur etc. ab und zum anderen von der speziellen Geometrie der Tensidmoleküle, insbesondere der Länge der Alkylkette und der genauen Hydrophilität der Kopfgruppe. In allen Fällen ist es jedoch so, dass der Mischungsentropieterm, der der Bildung von Mizellen entgegen wirkt, bis zu einer bestimmten Konzentration (der $c m c$ ) überwiegt, wodurch erst bei höherer Tensidkonzentration Mizellen gebildet werden. Diese cmc ist dabei umso niedriger, je länger die Alkylkette der Tensidmoleküle ist. Allgemein lassen sich die $c m c$ Werte von Molekülen mit der gleichen Kopfgruppe gemäß $\log _{10} c m c \approx k_{1} \cdot n_{C}+k_{2}$ (mit substanzklassenabhängigen Parametern $k_{1}$ und $k_{2}$ ) bestimmen, wobei $n_{C}$ die Anzahl der Kohlenstoffatome in der Alkylkette ist. In der Literatur gibt es darüber hinaus zahlreiche Bestrebungen, die $c m c$ eines Systems ausschließlich aus der Struktur der Moleküle zu bestimmen [57]- [59].

Die Bildung von Mizellen oberhalb der $c m c$ führt bei vielen (messbaren) physikalischen Größen zu teilweise deutlich sichtbaren Verhaltensänderungen bei eben jener Konzentration. Einige Verläufe sind in Abbildung 19 schematisch dargestellt. 


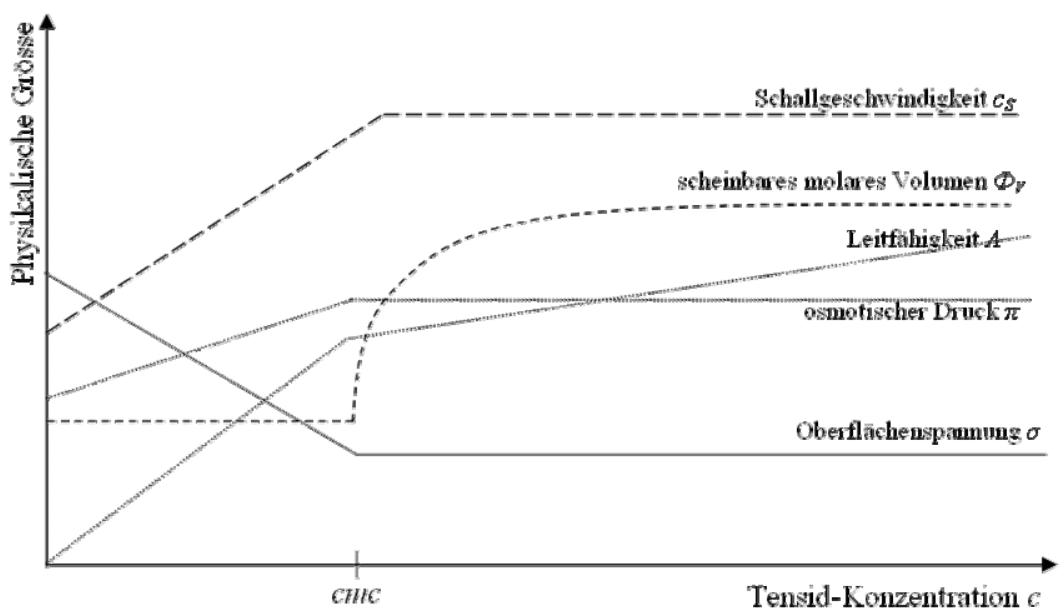

Abbildung 19: Schematischer Verlauf einiger physikalischer Größen von Tensid-Lösungen. Die Verläufe müssen nicht für alle Systeme zwingend die gezeigte Form haben - so nimmt die elektrische Leitfähigkeit nur für Lösungen ionischer Tenside den obigen Verlauf.

Dem charakteristischen Verlauf der obigen physikalischen Größen liegt dabei natürlich zugrunde, dass die Tensidmoleküle einen unterschiedlichen Beitrag zu den Größen liefern, abhängig davon, ob sie in monomerer Form vorliegen oder in Mizellen aggregiert sind. Die Konzentration der Monomere entspricht bis zur cmc der Gesamtkonzentration des Tensids und bleibt (bei nichtionischen Tensiden) oberhalb der $c m c$ konstant, während die Konzentration der mizellaren (also in Mizellen aggregierten) Tensidmoleküle unterhalb der cmc Null beträgt und oberhalb ebenfalls linear mit der Gesamtkonzentration ansteigt:

$$
\begin{aligned}
& c_{\text {Mono }}= \begin{cases}c_{G e s} & \text { für } c_{G e s} \leq c m c \\
c m c & \text { für } c_{G e s}>c m c\end{cases} \\
& c_{\text {Miz }}= \begin{cases}0 & \text { für } c_{G e s} \leq c m c \\
c_{G e s}-c m c & \text { für } c_{G e s}>c m c\end{cases}
\end{aligned}
$$
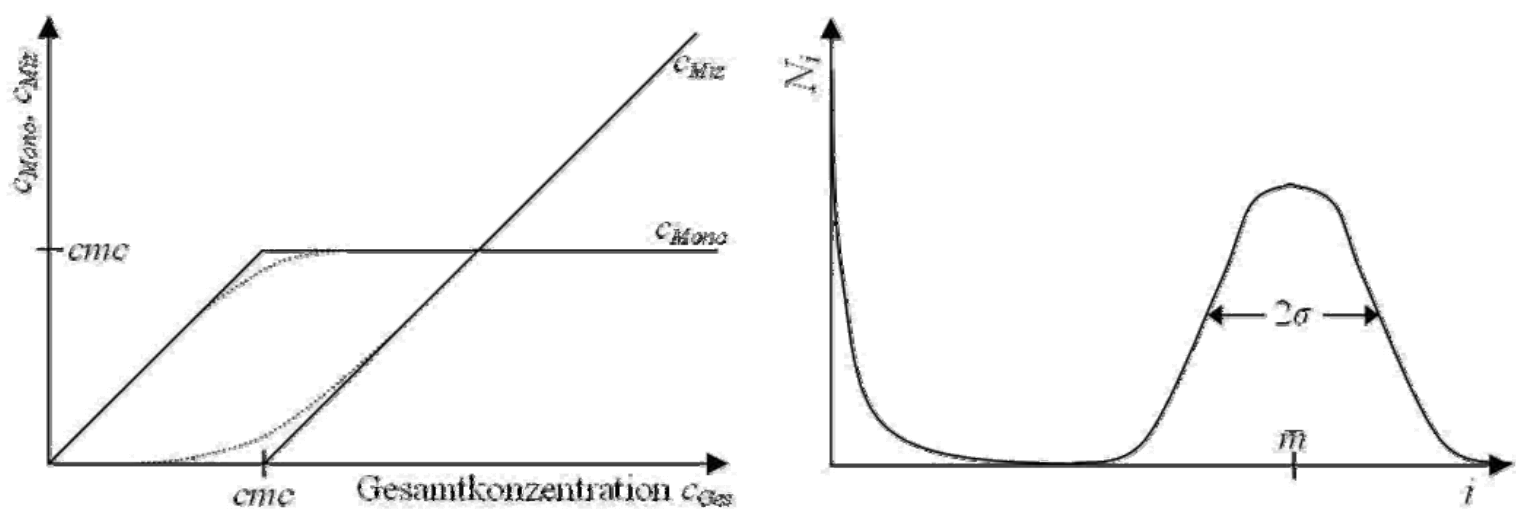

Abbildung 20: Schematischer Verlauf der Konzentration von monomeren und mizellaren Tensidmolekülen (links) und der Verteilung der Anzahl $N_{i}$ von Aggregaten aus $i$ Molekülen (rechts). Die durchgezogenen Linien in der linken Abbildung entsprechen dabei Gl. (3.1) und Gl. (3.2), während die gestrichelten Linien den realen Verlauf wiedergeben. 
Grundsätzlich können sich in Tensidlösungen oberhalb der $c m c$ Aggregate mit beliebig vielen Tensidmolekülen bilden, jedoch nimmt die Verteilung der Aggregatgrößen im Gleichgewicht nach theoretischen Überlegungen die in Abbildung 20 dargestellte Form an. Neben der großen Anzahl an Monomeren, $N_{1}$, bildet sich um eine mittlere Aggregationszahl $\bar{m}$ eine als gaußförmig angenommene Verteilung mit einer Standardabweichung $\sigma$, während der Oligomerbereich (also der Bereich der Dimere, Trimere, etc.) kaum besetzt ist. Dies liegt vor allem daran, dass der Zugewinn an Entropie durch die Abschirmung der Alkylkette bei einem Oligomer gegenüber einem Monomer zu gering ist. Die mittlere Aggregationszahl $\bar{m}$ ist abhängig vom speziellen System und ist vor allem geometrisch bedingt - ein Aggregat aus deutlich mehr als $\bar{m}$ Molekülen kann entweder nicht kugelförmig sein oder es müsste einen Hohlraum im Kugelinneren aufweisen, während ein Aggregat aus deutlich weniger als $\bar{m}$ Molekülen wie erwähnt keine vollständige Abschirmung der Alkylketten vom umgebenden Wasser mehr gewährleisten kann. Prinzipiell können folgende Aussagen über die mittlere Aggregationszahl $\bar{m}$ und das Verhalten mizellarer Systeme getroffen werden:

- Je länger die Alkylkette (bei gleicher Kopfgruppe) ist, desto schärfer sind die Übergänge im linken Teil der Abbildung 20 und damit auch die in Abbildung 19.

- Je länger die Alkylkette (bei gleicher Kopfgruppe) ist, desto größer ist die mittlere Aggregationszahl $\bar{m}$, da umso größere Mizellen gebildet werden können.

- Je kürzer die Alkylkette ist, desto mehr Oligomere befinden sich in der Lösung, insbesondere bereits unterhalb der $c m c$.

Um eine Normierung der Konzentration auf die $c m c$ zu erhalten, die bei unterschiedlichen Systemen über einige Größenordnungen variieren kann, definiert man praktischerweise eine reduzierte Konzentration $X$, so dass $X_{c m c}=0$ für alle unterschiedlichen Systeme gilt:

$$
X \equiv \frac{c-c m c}{c m c}
$$

Für die spezielle Aggregations- und Zerfalls-Kinetik von Mizellen lassen sich aus der in Abbildung 20 (rechts) gegebenen Verteilung der Aggregatgrößen folgende Überlegungen ableiten:

Statt eines kompletten Reaktionsschemas, in dem alle möglichen Aggregationsprozesse erfasst sind, also

$$
A_{i}+A_{j} \underset{k_{i, j}^{b}}{\stackrel{k_{i, j}^{f}}{\rightleftharpoons}} A_{i+j},
$$

reicht es, aufgrund der vernachlässigbar geringen Anzahl an Oligomeren die Aggregation eines beliebigen Aggregates mit einem Monomer zu betrachten:

$$
A_{1}+A_{i} \underset{k_{i}^{b}}{\stackrel{k_{i}^{f}}{\rightleftharpoons}} A_{i+1}
$$

Dieser Aggregations-Prozess (Gl. (3.5)), der sogenannte Monomeraustauschprozess, ist die in der Theorie der Mizellkinetik [8]-[13] als ,schneller Prozess' bezeichnete Abnahme/Zunahme der mittleren Aggregationszahl $\bar{m}$ bei konstanter Anzahl an Mizellen nach kleiner Auslenkung des Systems aus dem Gleichgewicht, z.B. durch eine Druckänderung. Da die Relaxationszeit dieses Prozesses gewöhnlicherweise in der Größenordnung von Nanose- 
kunden bis Mikrosekunden liegt, ist dieser Prozess mit der Ultraschallabsorptionsspektroskopie gut messbar. Parallel dazu läuft ein langsamerer Prozess ab, der Mizellbildungs-/-zerfallsprozess, bei dem Mizellen gebildet werden bzw. zerfallen, so dass die ursprüngliche mittlere Aggregationszahl $\bar{m}$ nahezu wieder hergestellt wird, sich die Anzahl an Mizellen aber verringert bzw. erhöht. Da dazu die Mizellen den dünn besiedelten Oligomerbereich ,durchlaufen' müssen, ist dieser Prozess wesentlich langsamer als der Monomeraustauschprozess - seine Relaxationszeit liegt für gewöhnlich im Bereich von Millisekunden bis zu Minuten und kann z.B. mit Drucksprungexperimenten nachgewiesen werden [60].

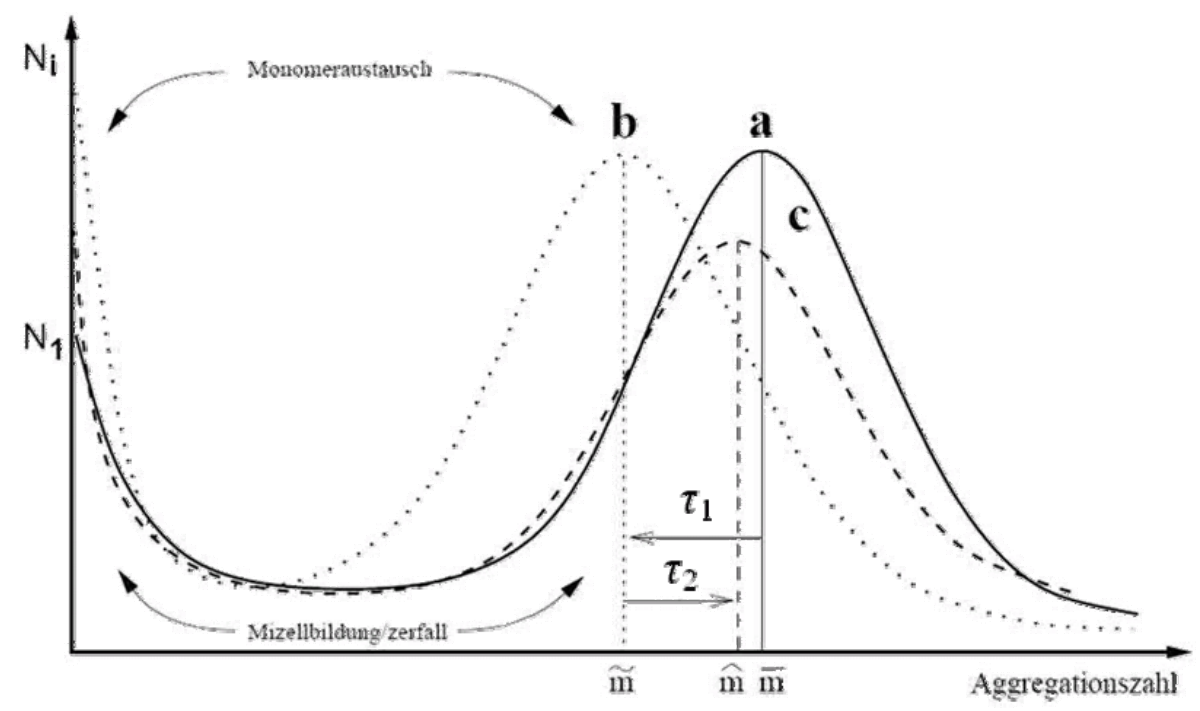

\begin{abstract}
Abbildung 21: Schematische Darstellung der beiden Prozesse der Mizellkinetik nach [8], entnommen aus [29]: Die Gleichgewichtsverteilung a wird durch eine Druckerhöhung gestört; das System reagiert auf die Änderung der äußeren Parameter und relaxiert über den Zwischenschritt b, in dem durch den schnellen Monomeraustauschprozess kleinere Aggregate vorliegen, in eine neue Gleichgewichtslage c, in der weniger Mizellen mit (nahezu) der ursprünglichen Größe vorliegen.
\end{abstract}

Aktuelle theoretische Überlegungen [61] sagen einen weiteren Relaxationsprozess voraus, bei dem die Breite der Mizellverteilung $\sigma$ relaxiert. Die Relaxationszeit $\tau$ dieses Prozesses wird je nach Tensidkonzentration in der Größenordnung der Relaxationszeit des Monomeraustauschprozesses (für kleine Konzentrationen) oder deutlich größer (für größere Konzentrationen) erwartet. Es ist allerdings davon auszugehen, dass eine solche $\sigma$ Relaxation, also eine Verbreiterung bzw. Verschmälerung der Mizellverteilung, kein nennenswertes Reaktionsvolumen aufweisen würde und damit in der Ultraschallabsorptionsspektroskopie nicht messbar wäre. Dementsprechend haben bisherige Ultraschallabsorptionsuntersuchungen an Tensid-Systemen einen solchen Prozess auch nicht detektiert [17], [62]. Weitere in mizellaren Lösungen bekannte Prozesse wie die Oligomerrelaxation [17], [48], [62] - [64], die begrenzte radiale Diffusion [48], die Alkylkettenisomerie [17], [48], [62], [63] oder die extrem langsame Relaxation nach großer Störung des Gleichgewichts [65] seien hier genau wie die langsame Relaxation des Mizellbildungs-/-zerfallsprozesses nur kurz erwähnt, da in der vorliegenden Arbeit ausschließlich der schnelle Monomeraustauschprozess gemessen wurde.

Für den mit der Ultraschallabsorptionsspektroskopie im Allgemeinen messbaren schnellen Monomeraustauschprozess hingegen gibt es theoretische Überlegungen von Teubner und Kahlweit [66] zur resultierenden Ultraschalldämpfung. Diese ergeben eine lineare Abhängigkeit der reziproken Relaxationszeit $\tau^{-1}$ von der reduzierten Konzentration $X$ und einen Anstieg der Amplitude, der schließlich in einen konstanten Wert übergeht: 


$$
\begin{aligned}
\tau^{-1} & =k_{b} \cdot\left(\frac{1}{\sigma^{2}}+\frac{X}{\bar{m}}\right) \\
A & =\frac{\pi \cdot(\Delta V)^{2} \cdot c m c \cdot c_{S}^{2} \cdot \rho}{R \cdot T} \cdot \frac{\frac{\sigma^{2}}{\bar{m}} \cdot X}{1+\frac{\sigma^{2}}{\bar{m}} \cdot X}
\end{aligned}
$$

Hierbei sind bereits zwei Annahmen des zugrundeliegenden Modells benutzt worden: Zum einen tragen nur jene Aggregationsvorgänge gemäß Gl. (3.5) merklich zur Ultraschallabsorption bei, bei denen $i \approx \bar{m}$ ist. In diesem Bereich ist die Rückreaktionskonstante von der Aggregatgröße unabhängig: $k_{b} \equiv k_{i}^{b}$ für $i \approx \bar{m}$. Zum anderen wird angenommen, dass das Reaktionsvolumen für alle Aggregatgrößen gleich ist: $\Delta V \equiv \Delta V_{i} \forall i$. Die Verläufe der beiden Größen sind schematisch in Abbildung 22 dargestellt.
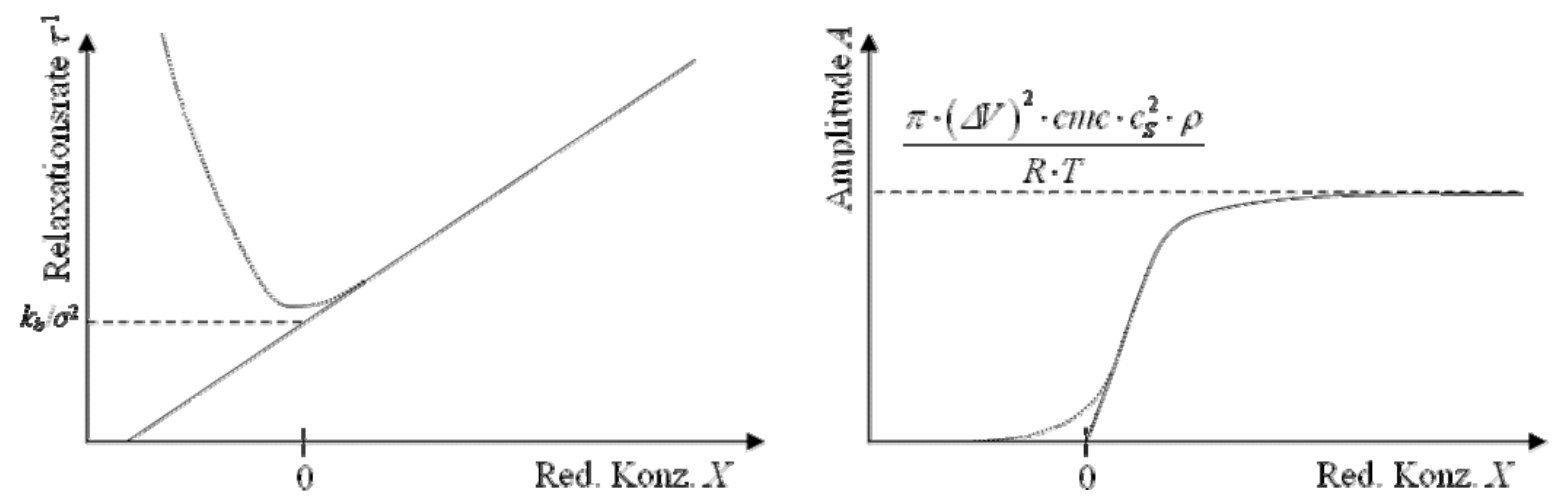

\begin{abstract}
Abbildung 22: Schematischer Verlauf der Relaxationsrate $\tau^{-1}$ (links) und der Amplitude $A$ (rechts) des Monomeraustauschprozesses über der reduzierten Konzentration $X$. Die durchgezogenen Linien entsprechen dem Modell nach Teubner und Kahlweit [66], die gepunkteten Linien der Erweiterung des Modells nach Telgmann [63].
\end{abstract}

Eine Erweiterung des Modells nach Telgmann [63] ergibt eine nicht verschwindende Amplitude des Monomeraustauschprozesses auch unterhalb der $c m c$ und einen Anstieg der Relaxationsrate $\tau^{-1}$ in diesem Bereich. Ein solcher Verlauf trägt der Tatsache Rechnung, dass bereits unterhalb der $c m c$ Aggregate aus Tensidmolekülen auftreten können, die allerdings kleiner als die voll ausgebildeten Mizellen oberhalb der $c m c$ sind. Da diese kleineren Aggregate einen größeren Diffusionskoeffizienten aufweisen, steigt die Wahrscheinlichkeit einer Begegnung zwischen einem Monomer und einem Aggregat und damit auch die Relaxationsrate.

Während sich aus der Auftragung der Relaxationsrate gegen die reduzierte Konzentration nach Gl. (3.6) also die Rückreaktionskonstante $k_{b}$ (bei Kenntnis von $\bar{m}$ ) aus der Steigung und die Mizellverteilungsbreite $\sigma$ aus dem Achsenabschnitt gewinnen lassen, erhält man aus dem Verlauf der Amplitude (bei Kenntnis der anderen Größen) nach Gl. (3.7) das Reaktionsvolumen des Monomeraustauschprozesses. Dieses entspricht gerade der Änderung der Hydrathülle eines Monomers bei Eintritt in eine Mizelle, wie in Abbildung 23 schematisch erklärt ist. Das Reaktionsvolumen des Monomeraustauschprozesses, also die Differenz zwischen der Hydrathülle des Tensidmoleküls im monomeren und im mizellaren Zustand, entspricht dort dem grün schraffierten Teil. 
Während das Volumen $V_{C}$ einer Alkylkette in einer Mizelle (also ohne das Reaktionsvolumen) nach Tanford [67] aus der Anzahl $n_{C}$ der Kohlenstoffatome des Alkyls gemäß

$$
V_{C}=\left(26.9 \cdot n_{C}+27.4\right) \cdot 10^{-3} \mathrm{~nm}^{3}
$$

abgeschätzt werden kann, ist eine solche Abschätzung für das Reaktionsvolumen nicht ohne weiteres möglich, da sowohl die nicht immer perfekte Abschirmung der Alkylketten in der Mizelle als auch eine mögliche Wasserstoffbrückenbindungsfähigkeit jener $\mathrm{CH}_{2}$-Gruppe, die sich am nächsten zur hydrophilen Tensidkopfgruppe befindet, in die genaue Größe mit eingeht. So findet beispielsweise Telgmann experimentell einen linearen $\mathrm{Zu}-$ sammenhang zwischen Reaktionsvolumen $\Delta V$ und Anzahl der Kohlenstoffatome $n_{C}, \Delta V \approx\left(1.56 \cdot n_{C}\right) \AA^{3}$, für die Reihe der nichtionischen Poly(ethylenglykol)alkylether [68], während bei der Reihe der kationischen Alkylammoniumchloride die gefundenen Werte deutlich kleiner sind und auch keinen so eindeutigen Verlauf über der Alkylkettenlänge aufweisen [29].
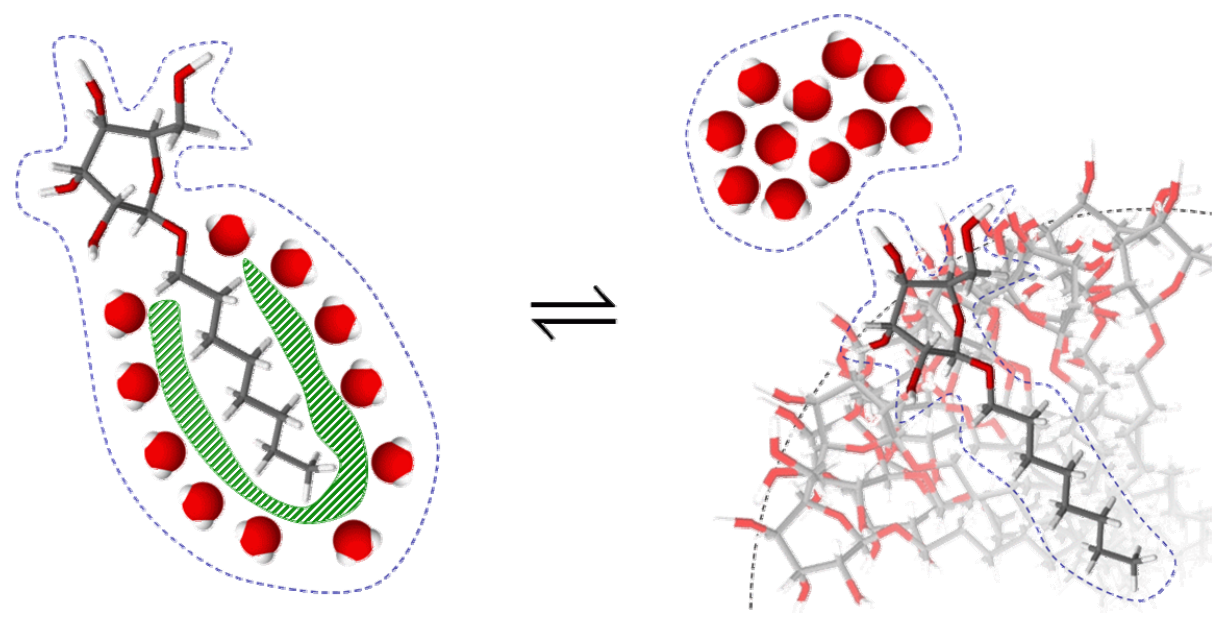

\begin{abstract}
Abbildung 23: Schematische Darstellung des Reaktionsvolumens des Monomeraustauschprozesses: Im monomeren Zustand stört die Alkylkette des Tensids die Dynamik des umgebenden Wassers (hier durch 11 Moleküle verdeutlicht), im mizellaren Zustand (rechts) hingegen nicht. Die Differenz der Gesamtvolumina der beeinflussten Wassermoleküle und des Tensidmoleküls (hier jeweils blau gestrichelt dargestellt) zwischen monomerem und mizellarem Zustand entspricht dem Reaktionsvolumen, bzw. der Hydrathüllenänderung (grün schraffiert).
\end{abstract}

Neben dem grundliegenden Ansinnen die Reaktionskonstanten $k_{b}$ und $k_{f}$, sowie das Volumen der Hydrathüllenänderung $\Delta V_{C}$ als wichtige Parameter der Mizellkinetik von Alkylglykosiden bestimmen zu können, finden sich beispielsweise in [60] und [69] weitere Gründe für die Relevanz der Mizellkinetik für technologische Prozesse. 


\subsection{Mizellgeometrie}

In den klassischen einfachen Modellen wird davon ausgegangen, dass sich oberhalb der $c m c$ kugelförmige Mizellen bilden, deren mittlere Aggregationszahl $\bar{m}$, und damit auch die mittlere Mizellgröße, von der Tensidkonzentration unabhängig ist. Dies ist allerdings gleich in zweierlei Hinsicht in den wenigsten Fällen zutreffend: Zum einen ist die konkrete Form der Mizellen stark von der Geometrie der einzelnen Moleküle abhängig. Israelachvili [70] hat in diesem Zusammenhang für eine quantitative Analyse der Molekülgeometrie den dimensionslosen Packungsparameter $P$ eingeführt, der berechnet werden kann gemäß

$$
P=\frac{V_{C}}{a_{0} \cdot l_{C}},
$$

wobei $V_{C}$ und $l_{C}$ das Volumen bzw. die Länge des hydrophoben Molekülteils bezeichnen und $a_{0}$ die Fläche des hydrophilen Molekülteils. Anhand von $P$ kann dann für ein Molekül abgeschätzt werden, was für eine Form von Aggregaten es bildet. In Tabelle 3.1 sind einige verschiedene Aggregationsformen in Abhängigkeit von $P$ nach [70] aufgelistet.

Zum anderen ist aus vielen theoretischen [71], [72] und experimentellen Arbeiten bekannt, dass sich die Form der Mizellen bei Variation der Temperatur, der Konzentration oder durch Zugabe von Salz ändern kann. Dies kann nach experimentellen Befunden sowohl an einer (temperaturabhängigen) ,zweiten cmc' ([73] - [75]) geschehen, also bei einer mehr oder weniger scharf bestimmbaren Konzentration, als auch als kontinuierliche Veränderung mit der Konzentration ([76], [77]). Auch die gleichzeitige Existenz von Aggregaten verschiedener Formen ist laut Literatur möglich ([74], [78]).

Da die genaue Geometrie erheblichen Einfluss auf die Solubilisierungsfähigkeit der Mizellen hat, ist die Frage, unter welchen Umständen welche Tensidmoleküle zu welcher Form aggregieren, ein sehr häufiges Thema theoretischer Überlegungen und experimenteller Untersuchungen. In Abbildung 24 sind einige der Mizellformen dargestellt, die in der Literatur behandelt werden .

\begin{tabular}{|c|c|c|c|c|c|c|c|c|}
\hline & \multirow[b]{2}{*}{ Rogel } & \multirow[b]{2}{*}{ Ellupsoid } & \multicolumn{3}{|c|}{ Sctrebchen (dieldx) } & \multicolumn{3}{|c|}{ Stabchen (mads) } \\
\hline & & & Scheibchen & Plstuchen & $\begin{array}{l}\text { Elliptisches } \\
\text { Passchen }\end{array}$ & Stabchen & Zylluader & $\begin{array}{l}\text { Spbero- } \\
\text { 2ylinder }\end{array}$ \\
\hline Aufucht & & & & & & & & \\
\hline Quesschaut & & & & & & & & 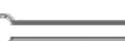 \\
\hline $\begin{array}{l}\text { 3-d- } \\
\text { Darrsellhuag }\end{array}$ & & & & & & & & \\
\hline
\end{tabular}

Abbildung 24: Schematische Darstellung unterschiedlicher Mizellformen. 


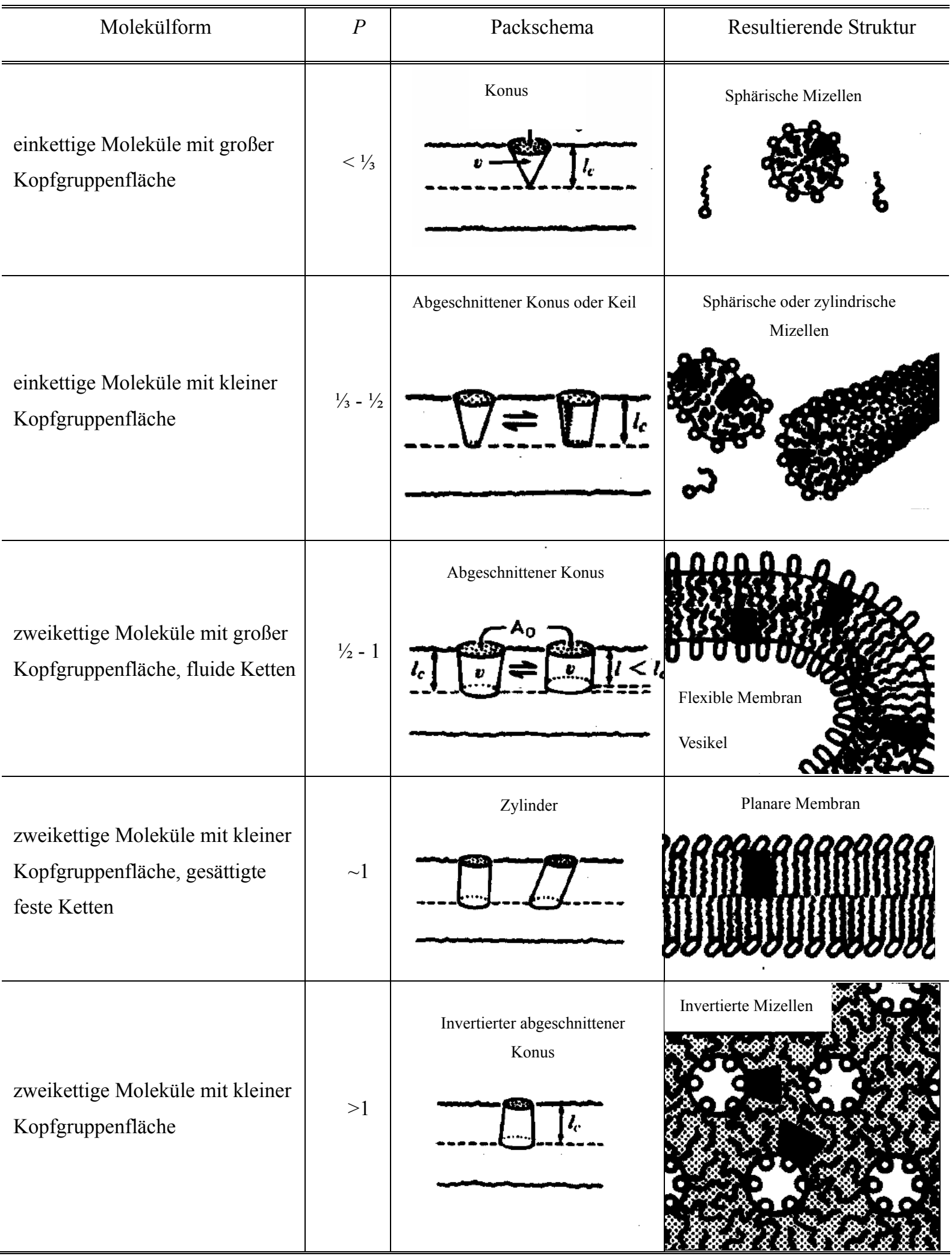

Tabelle 3.1: Schematische Darstellung verschiedener Aggregationsformen von amphiphilen Molekülen in Abhängigkeit von der Molekülform und dem daraus resultierenden Packungsparameter $P$ nach [70]. 
In der Realität werden in einer Flüssigkeit selbstverständlich immer Mizellen mit den unterschiedlichsten Formen existieren. Die entsprechenden geometrischen Modelle in Abbildung 24 sind daher zum einen als Idealisierung zu verstehen und zum anderen dienen die geometrischen Formen der einfacheren Berechnung des Zusammenhangs zwischen Mizellgeometrie und den messbaren Größen des Systems, wie z.B. Viskosität, statische Elektrizitätszahl etc. [105], [106]. Molekulardynamiksimulationen ergeben möglicherweise ein etwas realistischeres Bild von der Mizellform. In Abbildung 25 sind eine $\mathrm{C}_{8} \mathrm{G}_{1}$-Mizelle aus 20 Monomeren (oben) und eine aus 50 Monomeren (unten) aus eben solch einer Simulation [107] dargestellt.
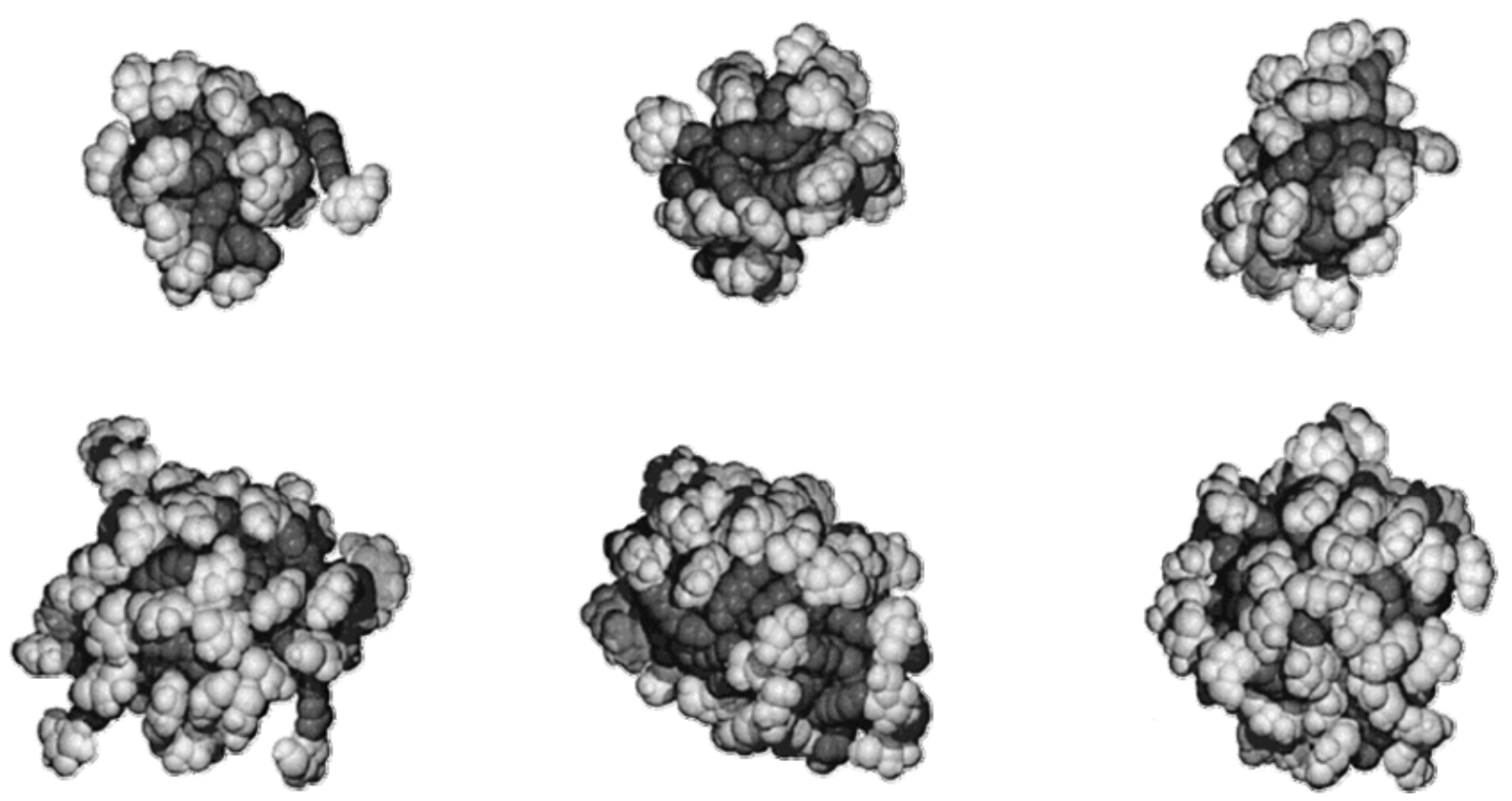

Abbildung 25: Schematische Darstellung einer $C_{8} G_{1}-$ Mizelle als Ergebnis einer Molekulardynamiksimulation, entnommen aus [107]: Oben ist ein 20mer dargestellt, unten ein 50mer. Dunkelgraue Atome sind Kohlenstoffatome des Alkylteils, hellgraue Atome sind Kohlenstoff-, Wasserstoff- oder Sauerstoffatome der Kopfgruppe. Von links nach rechts sind jeweils Schnappschüsse zu verschiedenen Zeiten der Simulation dargestellt. 


\subsection{Mizellkinetik und -geometrie von Alkylglykosiden}

Die im vorigen Kapitel bereits erwähnten experimentellen Arbeiten zur Untersuchung der Mizellgeometrie in Abhängigkeit von Konzentration, Temperatur oder bei Zugabe von Salzen oder anderen Substanzen basieren auf den unterschiedlichsten Methoden wie Kleinwinkel-Röntgen- (SAXS, Small-Angle X-Ray Scattering), Kleinwinkel-Neutronen- (SANS, Small-Angle Neutron Scattering) oder Lichtstreuung (SLS, Static Light Scattering), Fluoreszenzmethoden (SSFQ, Steady-State Fluorescence Quenching; TRFQ, Time-resolved Fluorescence Quenching) oder gravimetrischen Methoden (AUC, Analytical Ultracentrifugation; SedVel, Sedimentation Velocity Measurement). In Tabelle 3.2 sind für die beiden sehr intensiv untersuchten Alkylglykoside $\mathrm{C}_{8} \mathrm{G}_{1}$ und $\mathrm{C}_{12} \mathrm{G}_{2}$ einige Ergebnisse aus solchen Messungen aufgelistet. Aus den teilweise sehr unterschiedlichen Werten für die mittlere Aggregationszahl $\bar{m}$ wird ersichtlich, dass eine Bestimmung der Reaktionskonstanten $k_{b}$ gemäß Gl. (3.6) aus den Relaxationszeiten $\tau$ der Ultraschallabsorptionsmessungen an den Alkylglykosid-Lösungen sich als schwierig gestalten wird, da die mittlere Aggregationszahl $\bar{m}$ für die untersuchten Systeme zum einen nicht als konzentrationsunabhängig und zum anderen trotz der zahlreichen aufgeführten Werte nicht als bekannt vorausgesetzt werden kann. Daher wird es bei der Auswertung der Relaxationszeiten und -Amplituden des Monomeraustauschprozesses eher interessant sein, welche der unterschiedlichen Ergebnisse zur Mizellgeometrie von Alkylglykosiden durch die Daten dieser Arbeit unterstützt werden.

Zur Kinetik des Monomeraustauschprozesses von Alkylglykosiden sind in der Literatur lediglich Untersuchungen an einer Substanz, dem in dieser Arbeit ebenfalls untersuchten $\mathrm{C}_{8} \mathrm{G}_{1}$, durch Ultraschallabsorptionsspektroskopie zu finden [84]. In dieser Arbeit wurde bei vier Konzentrationen die Ultraschalldämpfung von $C_{8} G_{1}$ Lösungen zwischen etwa $0.5 \mathrm{MHz}$ und $100 \mathrm{MHz}$ bestimmt und mit einer Debye-Spektralfunktion angepasst. Aus den resultierenden Amplituden und Relaxationszeiten wurden gemäß Gl. (3.6) und Gl. (3.7) das Reaktionsvolumen $(\Delta V=8.2 \mathrm{~mL} / \mathrm{mol})$ und die Reaktionskonstanten $\left(k_{f}=5.6 \cdot 10^{-9} \mathrm{~L} \cdot \mathrm{mol}^{-1} \cdot \mathrm{s}^{-1}, k_{b}=1.4 \cdot 10^{-8} \mathrm{~s}^{-1}\right)$ bestimmt. Diese Daten werden zum Vergleich bei der Analyse des Monomeraustauschprozesses (Kapitel 5.1) herangezogen werden. 


\begin{tabular}{|c|c|c|c|c|c|c|c|}
\hline Substanz & $\begin{array}{c}c \\
{[\mathrm{mmol} / \mathrm{L}]}\end{array}$ & Form & $\begin{array}{c}R_{1} \\
{[\AA]}\end{array}$ & $\begin{array}{c}R_{2} \\
{[\AA]}\end{array}$ & $\begin{array}{c}\text { Mittl. } \\
\text { Aggr.-zahl } \\
\bar{m} \\
\end{array}$ & Methode & Referenz \\
\hline \multirow[t]{2}{*}{$\mathrm{C}_{8} \mathrm{G}_{1}$} & 34 & Zylinder & 12 & 11 & 24 & SANS & \multirow[t]{2}{*}{ [79] } \\
\hline & 68 & Zylinder & 14 & 11 & 51 & SAXS & \\
\hline \multirow[t]{2}{*}{$\mathrm{C}_{8} \mathrm{G}_{1}$} & 100 & - & - & - & 72 & SSFQ & \multirow[t]{2}{*}{ [80] } \\
\hline & 100 & - & - & - & 105 & TRFQ & \\
\hline \multirow{2}{*}{$\mathrm{C}_{8} \mathrm{G}_{1}$} & 29 & - & - & - & 54 & SSFQ & \multirow[t]{2}{*}{ [81] } \\
\hline & 55 & - & - & - & 105 & SSFQ & \\
\hline \multirow[t]{2}{*}{$\mathrm{C}_{8} \mathrm{G}_{1}$} & $\sim 30$ & - & - & - & 70 & SedVel & \multirow[t]{2}{*}[76]{} \\
\hline & $\sim 340$ & - & - & - & 100 & SedVel & \\
\hline \multirow{4}{*}{$\mathrm{C}_{8} \mathrm{G}_{1}$} & 26 & Ellipsoid & 45 & 13 & 66 & SANS & \multirow{4}{*}[82]{} \\
\hline & 50 & Ellipsoid & 61 & 13 & 76 & SANS & \\
\hline & 150 & Ellipsoid & 80 & 12 & - & SANS & \\
\hline & 300 & Ellipsoid & 73 & 11 & - & SANS & \\
\hline $\mathrm{C}_{8} \mathrm{G}_{1}$ & 100 & Zylinder & 96 & 13 & 90 & SANS/SAXS & [83] \\
\hline $\mathrm{C}_{8} \mathrm{G}_{1}$ & 60 & - & - & - & 92 & TRFQ & {$[84]$} \\
\hline $\mathrm{C}_{8} \mathrm{G}_{1}$ & 24-41 & - & - & - & 76 & SLS & {$[85]$} \\
\hline \multirow{7}{*}{$\mathrm{C}_{8} \mathrm{G}_{1}$} & 34 & - & - & - & 75 & SedVel & \multirow{7}{*}{ [87] } \\
\hline & 68 & - & - & - & 103 & SedVel & \\
\hline & 67.8 & Kugel & 25.3 & 25.3 & 27 & SANS & \\
\hline & 92.2 & Kugel & 24.2 & 24.2 & 25 & SANS & \\
\hline & 96.5 & Kugel & 24.1 & 24.1 & 26 & SAXS & \\
\hline & 97.4 & Kugel & 24.3 & 24.3 & 26 & SAXS & \\
\hline & 188 & Kugel & 21.4 & 21.4 & 22 & SANS & \\
\hline \multirow{2}{*}{$\mathrm{C}_{12} \mathrm{G}_{2}$} & 50 & Ellipsoid & 29 & 21 & 76 & SANS & \multirow[t]{2}{*}{ [88] } \\
\hline & 150 & Ellipsoid & 33 & 21 & 88 & SANS & \\
\hline \multirow[t]{2}{*}{$\mathrm{C}_{12} \mathrm{G}_{2}$} & 0.36 & Kugel & 31 & 30 & 94 & AUC & \multirow[t]{2}{*}{ [89] } \\
\hline & $1-100$ & Ellipsoid & 42 & 35 & 148 & AUC & \\
\hline $\mathrm{C}_{12} \mathrm{G}_{2}$ & $20-40$ & Oblate/Ellipse & 34 & 20 & 132 & SANS+SAXS & {$[90]$} \\
\hline \multirow{6}{*}{$\mathrm{C}_{12} \mathrm{G}_{2}$} & 0.27 & Rods/Disks & - & - & 69 & SSFQ & \multirow{6}{*}{ [91] } \\
\hline & 0.62 & Rods/Disks & - & - & 96 & SSFQ & \\
\hline & 1.47 & Rods/Disks & - & - & 86 & SSFQ & \\
\hline & 9.75 & Rods/Disks & - & - & 114 & SSFQ & \\
\hline & 30 & Rods/Disks & - & - & 111 & SSFQ & \\
\hline & 134 & Rods/Disks & - & - & 114 & SSFQ & \\
\hline \multirow{3}{*}{$\mathrm{C}_{12} \mathrm{G}_{2}$} & 5 & Ellipsoid & 30 & 26 & 133 & AUC & \multirow{3}{*}{ [92] } \\
\hline & 25 & Ellipsoid & 32 & 27 & 122 & AUC & \\
\hline & 50 & Ellipsoid & 32 & 27 & 112 & AUC & \\
\hline
\end{tabular}

Tabelle 3.2: Übersicht über einige Ergebnisse von Messungen zur Geometrie von AlkylglykosidMizellen aus der Literatur. $R_{1}$ und $R_{2}$ sind dabei der Radius der großen bzw. kleinen Halbachse bei Ellipsoiden; bei Zylindern ist $R_{1}$ die Länge. Die Abkürzungen der Methoden sind im Text erläutert. 


\subsection{Cyclodextrine}

In der vorliegenden Arbeit wurden neben reinen Alkylglykosid-Lösungen auch Mischungen mit $\alpha$-Cyclodextrin untersucht. Dabei handelt es sich um einen zyklischen Zusammenschluss $\alpha-(1,4)$-verbundener Glukosemoleküle, wie in Abbildung 26 schematisch dargestellt. Das vorgestellte $\alpha$ gibt dabei an, dass es sich um das kleinstmögliche Cyclodextrin handelt, nämlich das aus 6 Glukose-Molekülen aufgebaute - ein vorgestelltes $\beta$ oder $\gamma$ entspräche dementsprechend 7 bzw. 8 Glukose-Molekülen. Aus Computersimulationen und experimentellen Untersuchungen ist bekannt, dass Cyclodextrine die Form eines Kegelstumpfes haben, wobei das Innere leicht hydrophob ist. Dies führt in wässriger Lösung dazu, dass alle Substanzen, die in das entsprechende Innenvolumen hineinpassen, mit dem Cyclodextrin komplexieren können, um (ähnlich wie bei der Mizellbildung) die Bereiche hydrophober Hydratation zu minimieren. Dieser Effekt wird unter anderem in geruchsvernichtenden Mitteln ausgenutzt, aber auch in zahllosen weiteren Bereichen der Industrie, Pharmazie und Forschung [93]. Weiterhin stellt die Komplexierung von Cyclodextrinen mit anderen Substanzen ein Modellsystem für kompliziertere Komplexe wie Enzym-Substrat-Komplexe dar.
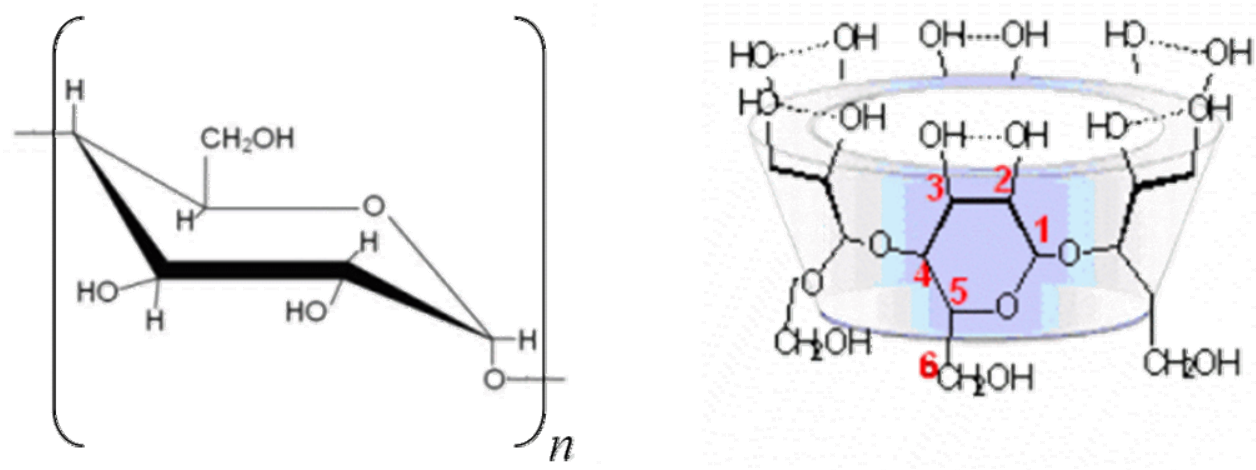

Abbildung 26: Schematische Darstellung von Cyclodextrin. In der Strukturformel (links) ist $n=6,7,8$ für $\alpha$-, $\beta$ - oder $\gamma$-Cyclodextrin, in der dreidimensionalen Darstellung von $\alpha$-Cyclodextrin (rechts) ist die Kegelstumpfform erkennbar.

In der Literatur existieren bereits zahlreiche Ultraschallabsorptionsuntersuchungen an reinen CyclodextrinLösungen [49], [94] und an Mischungen mit z.B. Kaliumiodid [49], Acetylsalicylsäure (Aspirin) [95], Alkoholen [96] oder Carboxylsäuren [97].

In beiden Untersuchungen an reinen $\alpha$-Cyclodextrin-Lösungen weisen die Ultraschallabsorptionsspektren einen Relaxationsprozess bei etwa 3 ns [49] bzw. 7.5 ns [94] auf, der, wie bei den Mono- und Disacchariden, der (teilweisen) Rotation der exozyklischen Gruppe um den Bindungswinkel $\omega$ zugeordnet wird (siehe Kapitel 3.2.2). In Abbildung 26 wird deutlich, dass die exozyklischen Gruppen vermutlich nicht völlig in ihrer Bewegung gestört werden, da sie sich alle am äußeren Rand des Moleküls befinden. Allerdings lässt das Auffinden einer deutlich kleineren Relaxationsamplitude pro exozyklischer Gruppe gegenüber z.B. Glukose und Maltose vermuten, dass die einzelnen Hydroxymethylgruppen sich gegenseitig sterisch behindern und damit die einzelnen Rotamerverteilungen deutlich beeinflussen [49].

Weiterhin tritt auch in beiden erwähnten Untersuchungen ein Relaxationsprozess mit einer konzentrationsunabhängigen Relaxationszeit von etwa 21 ns [49] bzw. 110 ns [94] auf, der einer teilweisen Verdrehung der 46 
einzelnen Glukoseringe gegeneinander zugeordnet wird. Dies geschieht natürlich wiederum an den glykosidischen Bindungswinkeln $\varphi$ und $\psi$ (siehe Kapitel 3.2.1), wobei im Vergleich zu Mono- und Disacchariden eine evident verschiedene geometrische Situation durch den zyklischen Zusammenschluss der Glukoseringe vorliegt, so dass ein direkter Vergleich der Relaxationszeiten oder -amplituden mit denen der $\varphi$-/ $\psi$-Rotation von Monound Disacchariden nicht sinnvoll erscheint.

Bei den Untersuchungen an wässrigen Lösungen aus Cyclodextrin und einer weiteren Substanz ergibt sich die zusätzliche Fragestellung nach einer möglichen Komplexierung. In den oben angegebenen Arbeiten tritt jeweils ein weiterer Relaxationsprozess in den Ultraschallabsorptionsspektren auf, der eben der Komplexierung zugeschrieben wird. Aus den Relaxationszeiten und -amplituden dieser Prozesse können mithilfe von Gl. (2.26) und (2.27) dann das Reaktionsvolumen $\Delta V_{S}$ und die Gleichgewichtskonstante $K$ gewonnen werden.

Im speziellen Falle von Untersuchungen an Lösungen aus Cyclodextrin und einem Tensid stellt sich die Frage, wie das System auf die zusätzliche Möglichkeit der Vermeidung hydrophober Hydratation reagiert, also ob die Zugabe von Cyclodextrin die Mizellkinetik beeinflusst. In der Literatur existieren dazu z.B. Untersuchungen an Lösungen von Cyclodextrin und $\mathrm{C}_{12} \mathrm{E}_{4}$ (Tetraethylenglykoldodecylether) [98], SDS (Natriumdodecylsulfat) [99] oder auch Alkylglykosiden [100], [101].

In den älteren Untersuchungen zu diesem Thema wird stets davon ausgegangen, dass bei schrittweiser Erhöhung der Tensidkonzentration zunächst nur Cyclodextrin-Tensid-Komplexe gebildet werden, bis alle CyclodextrinMoleküle komplexiert sind und erst dann eine Mizellisierung stattfindet. Aktuellere Untersuchungen hingegen zeigen, dass in allen untersuchten Systemen immer ein gewisser Prozentsatz an nicht komplexierten Cyclodextrin-Molekülen vorliegt, der von der Kopfgruppe des zugegebenen Tensids, der Art des Cyclodextrins $(\alpha, \beta$ oder $\gamma$ ) und insbesondere von der Kettenlänge des Tensids abhängt [102], [103]. Das genaue Verständnis des Zusammenspiels der beiden konkurrierenden Vorgänge Mizellisierung und Cyclodextrin-Tensid-Komplexierung ist von großer Relevanz für die Produktion von Waschmitteln, Seifen, etc., in denen neben Tensiden auch Cyclodextrine eingesetzt werden, um zum einen ein vorzeitiges Ausdampfen zugegebener Parfüme zu verhindern und zum anderen die geruchsvernichtende Wirkung der Cyclodextrin-Moleküle zu gewährleisten. 


\subsection{Substanzen und Präparation der Lösungen}

In der vorliegenden Arbeit wurden die in Tabelle 3.3 aufgeführten Substanzen untersucht. Die Reinheit betrug nach Herstellerangaben für alle Substanzen mindestens 98\%. Daher wurden alle Substanzen ohne weitere Reinigung benutzt.

\begin{tabular}{|c|c|c|c|c|c|}
\hline Name & $\begin{array}{l}\text { Abkür- } \\
\text { zung }\end{array}$ & $\begin{array}{c}\text { Molekulargewicht } \\
\qquad \mathrm{g} / \mathrm{mol}]\end{array}$ & $\begin{array}{l}\text { Mittl. Aggre- } \\
\text { gationszahl } \bar{m}\end{array}$ & $\begin{array}{c}c m c \\
{\left[10^{-3} \mathrm{~mol} / \mathrm{L}\right]}\end{array}$ & Bezugsquelle \\
\hline Glukose & Glu & 180.2 & - & - & 1 \\
\hline Maltose & Mal & 342.3 & - & - & 1 \\
\hline$\alpha$-Cyclodextrin & $\alpha-\mathrm{CD}$ & 972.0 & - & - & 1 \\
\hline Hexyl- $\beta$-D-Glukopyranosid & $\mathrm{C}_{6} \mathrm{G}_{1}$ & 264.3 & k.A. & $250^{[104]}$ & 2 \\
\hline Heptyl- $\beta$-D-Glukopyranosid & $\mathrm{C}_{7} \mathrm{G}_{1}$ & 278.3 & k.A. & $70^{[104]}$ & 2 \\
\hline Octyl- $\beta$-D-Glukopyranosid & $\mathrm{C}_{8} \mathrm{G}_{1}$ & 292.4 & $27-103^{[87]}$ & $18-26^{[87]}$ & 2 \\
\hline Octyl- $\beta$-D-Galaktopyranosid & $\mathrm{C}_{8} \mathrm{Gal}$ & 292.4 & k.A. & $29.5^{[104]}$ & 2 \\
\hline Nonyl- $\beta$-D-Glukopyranosid & $\mathrm{C}_{9} \mathrm{G}_{1}$ & 306.4 & k.A. & $6.5^{[104]}$ & 2 \\
\hline Hexyl- $\beta$-D-Maltopyranosid & $\mathrm{C}_{6} \mathrm{G}_{2}$ & 426.4 & k.A. & $210^{[104]}$ & 2 \\
\hline Octyl- $\beta$-D-Maltopyranosid & $\mathrm{C}_{8} \mathrm{G}_{2}$ & 454.4 & $47^{[104]}$ & $19.5^{[104]}$ & 2 \\
\hline Nonyl- $\beta$-D-Maltopyranosid & $\mathrm{C}_{9} \mathrm{G}_{2}$ & 468.7 & $55^{[104]}$ & $6^{[104]}$ & 2 \\
\hline Decyl- $\beta$-D-Maltopyranosid & $\mathrm{C}_{10} \mathrm{G}_{2}$ & 482.6 & $69^{[104]}$ & $1.8^{[104]}$ & 2 \\
\hline Decyl- $\alpha-D-M a l t o p y r a n o s i d$ & $\alpha-\mathrm{C}_{10} \mathrm{G}_{2}$ & 482.6 & k.A. & k.A. & 2 \\
\hline
\end{tabular}

Tabelle 3.3: Auflistung der untersuchten Substanzen und einiger ihrer Basisparameter. k.A. bedeutet, dass in der Literatur keine Angaben auffindbar sind. Bezugsquellen: 1: Sigma-Aldrich, München; 2: Anatrace Inc., Ohio, USA.

Von allen Substanzen wurde jeweils zunächst eine Stammlösung mit der höchsten zu untersuchenden Konzentration $c_{\text {Stamm }}$ angesetzt, indem die gelieferte Substanz (in allen Fällen in Pulverform vorliegend) unter Stickstoffatmosphäre abgewogen wurde (Sartorius BP 3100s, $\Delta m=0.01 \mathrm{~g}$ ) und dann in einem Messzylinder bidestilliertes, milliporegereinigtes, gefiltertes Wasser auf das Volumen $V=m_{\text {Subst }} / M W_{\text {Subst }} c_{\text {Stamm }}$ aufgefüllt wurde. Bei einigen der Substanzen erwies sich das Lösen des Pulvers im Wasser als schwierig - in diesen Fällen wurde zunächst etwas weniger Wasser zugegeben, das Pulver dann durch Rühren und/oder Erwärmen in Lösung gebracht und erst danach das restliche Wasser zugegeben. Sämtliche Lösungen wurden zwischen den Messungen im Kühlschrank gelagert. Alle benutzten Messkolben, Messzylinder, Reagenzgläser etc. wurden vor der Benutzung zunächst mehrfach mit reinem Wasser und Aceton gespült und anschließend mit Stickstoff getrocknet. Jene Kolben, in denen sich Messlösungen befanden, wurden mit Stopfen und Parafilm sorgfältig luftdicht verschlossen und 48 
nur für die jeweilige Entnahme der Messlösungen geöffnet. Zudem wurden alle Messlösungen regelmäßig auf optische Veränderungen begutachtet, allerdings konnten solche während der Messungen nicht beobachtet werden.

Wegen der teilweise immens hohen Kosten der reinen Alkylglykoside wurde so verfahren, dass die Messungen zunächst an der Stammlösung vorgenommen wurden und diese dann mehrfach verdünnt wurde. Um Verschmutzungen oder Konzentrationsänderungen durch die Rückgewinnung der einzelnen Lösungen aus den Messapparaturen erkennen bzw. ausschließen zu können, wurde jeweils ein Rest der Stammlösung aufbewahrt. Dieser wurde dann am Ende einer Messreihe auf die geringste untersuchte Konzentration verdünnt um sie mit der ,benutzten' Lösung zu vergleichen. Bei Messungen der Dichte (Anton Paar Densitometer DMA 5000, $\Delta \rho=10^{-6} \mathrm{~g} / \mathrm{mL}$ ) konnten allerdings keine signifikanten Unterschiede zwischen den ,benutzten' Messlösungen und den Kontrolllösungen gefunden werden. 



\section{Ergebnisse und Auswertung der Spektren}

Die Auswertung der Ultraschallabsorptionsspektren erfolgte durch Anpassung einer Funktion gemäß Gl. (2.21) an die Messdaten. Dazu wurde jeweils ein Marquardt-Algorithmus der kleinsten Fehlerquadrate benutzt, der die reduzierte Varianz für die Beschreibung eines Satzes zusammengehöriger Messpunkte $\left(x_{i}, y_{i}\right)$ durch eine Funktion $F$ minimiert:

$$
\chi^{2}=\frac{1}{N-k-1} \cdot \sum_{i=1}^{N}\left(\frac{y_{i}-F\left(x_{i}\right)}{\Delta y_{i}}\right)^{2} \rightarrow \min
$$

Dabei ist $N$ die Anzahl der Messpunkte, $k$ die Anzahl der freien Parameter und $\Delta y_{i}$ der absolute Fehler des Messpunktes $y_{i}$.

Grundsätzlich wurde bei den Anpassungen so verfahren, dass das Ultraschallabsorptionsspektrum zunächst nur mit einer Debye-Spektralfunktion angepasst wurde. Trat dabei eine signifikante und vor allem systematische Abweichung der Messpunkte von der Funktion auf, wurde die Anzahl der Debye-Terme sukzessive erhöht, bis aus einer weiteren Erhöhung keine Verbesserung der Anpassung mehr resultierte. Mit diesem Verfahren kann eine Überparametrisierung der Spektren vermieden werden.

Bei vielen im Folgenden aufgeführten Spektren ist anzumerken, dass wegen der teilweise sehr geringen Tensidkonzentrationen nur sehr kleine Amplituden der Debye-Spektralfunktionen auftraten. Die dementsprechend geringe Exzessdämpfung weist daher in diesen Fällen eine vergleichsweise hohe Streuung der Messpunkte auf. In einigen Fällen mussten daher Parameter bei der Anpassrechnung festgehalten werden - diese sind in den jeweiligen Parametertabellen durch einen Stern gekennzeichnet.

Aus Platzgründen werden nicht alle aufgenommenen Spektren dargestellt, sondern pro System jeweils zwei bis vier. Da in der Darstellung $\left(\alpha / f^{2}\right)$ einige der Debye-Spektralfunktionen nicht gut erkennbar sind, werden alle Darstellungen zusätzlich noch in der $(\alpha \lambda)_{\text {exz }}$-Form gegeben. Letztere sind jeweils in skalentreuer Auftragung dargestellt, während dies bei den $\left(\alpha / f^{2}\right)$-Spektren wegen des vergleichsweise hohen Bereichs der $\left(\alpha / f^{2}\right)$-Werte innerhalb der einzelnen Abbildungen nicht möglich ist. Die $\left(\alpha / f^{2}\right)$-Spektren sind trotz dieser beiden Nachteile aufgeführt, da sie reine, unverfälschte Messdaten beinhalten, während bei den $(\alpha \lambda)_{\mathrm{exz}}$-Spektren der aus der Anpassrechnung resultierende Hintergrundsanteil $B \cdot f$ abgezogen wurde.

Weiterhin sind in beiden Auftragungen alle Abbildungen doppelt logarithmisch gegeben und alle Ordinaten sind mit dem Faktor $10^{-3}\left((\alpha \lambda)_{\mathrm{exz}}\right.$-Spektren) bzw. $10^{-15}\left(\left(\alpha / f^{2}\right)\right.$-Spektren) skaliert, damit die einzelnen Abbildungen untereinander besser vergleichbar sind.

In den Tabellen sind die aus den Anpassungen resultierenden Fehler der Parameter mit angegeben. Der absolute Fehler der Dichtemessungen beträgt $\Delta \rho=10^{-5} \mathrm{~g} / \mathrm{mL}$ und der Fehler der Schallgeschwindigkeitswerte kann auf $\Delta c_{S}=1 \mathrm{~m} / \mathrm{s}$ abgeschätzt werden. 


\subsection{Glukose}

In der Literatur finden sich Ultraschallabsorptionsspektren von $\beta$-D-Glukose-Lösungen bei vier verschiedenen Konzentrationen $\left(300 \mathrm{mmol} / \mathrm{L}, 500 \mathrm{mmol} / \mathrm{L}, 800 \mathrm{mmol} / \mathrm{L}, 1000 \mathrm{mmol} / \mathrm{L}\right.$, alle bei $25^{\circ} \mathrm{C}$ [108]). Da die Messungen an Alkylglykosid-Lösungen zum größten Teil bei geringeren Konzentrationen durchgeführt wurden, wurden zum Zwecke einer besseren Vergleichbarkeit auch die Spektren von Glukose-Lösungen bei $100 \mathrm{mmol} / \mathrm{L}$ und 200 mmol/L aufgenommen. Beide Spektren sind in Abbildung 27 in der Form $\left(\alpha / f^{2}\right)$ und in Abbildung 28 in der Form $(\alpha \lambda)_{\mathrm{exz}}$ dargestellt.

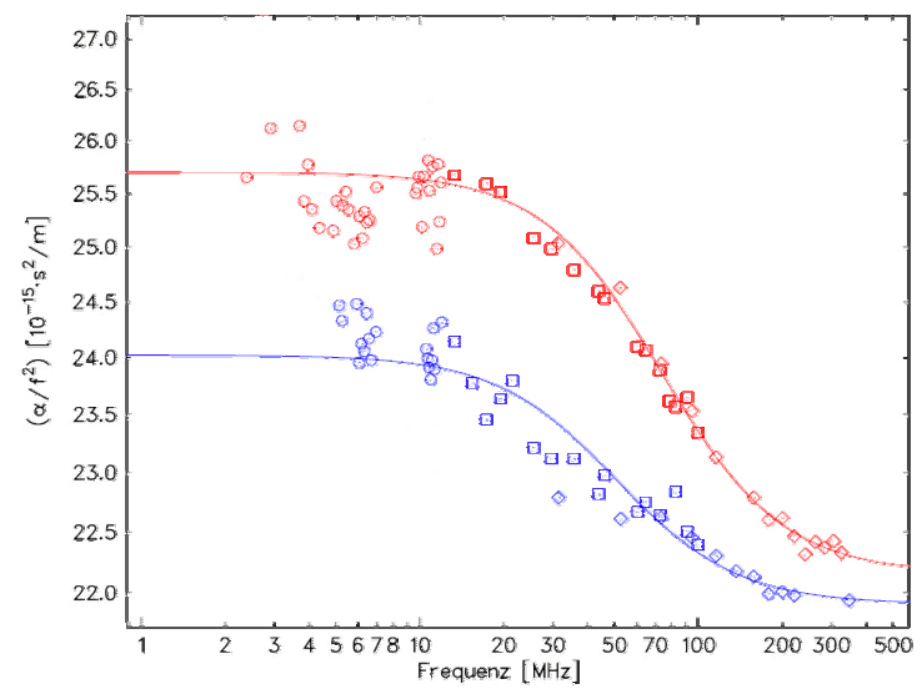

Abbildung 27: Ultraschallabsorptionsspektren von Glukose-Lösungen der Konzentrationen 100 $\mathrm{mmol} / \mathrm{L}$ (blau) und $200 \mathrm{mmol} / \mathrm{L}$ (rot) in der $\left(\boldsymbol{\alpha} / \boldsymbol{f}^{2}\right)$-Darstellung. Hier und im Folgenden: Die unterschiedlichen Symbole stehen für die benutzten Messzellen: $\triangle, 1-\mathrm{MHz}-$ Resonator; $\bigcirc, 8-\mathrm{MHz}-\mathrm{Resonator}$; $\square$, tieffrequente Pulszelle; $\diamond$, hochfrequente Pulszelle; die durchgezogenen Kurven sind die Graphen der jeweiligen Debye-Spektralfunktionen (Gl. (2.21)).

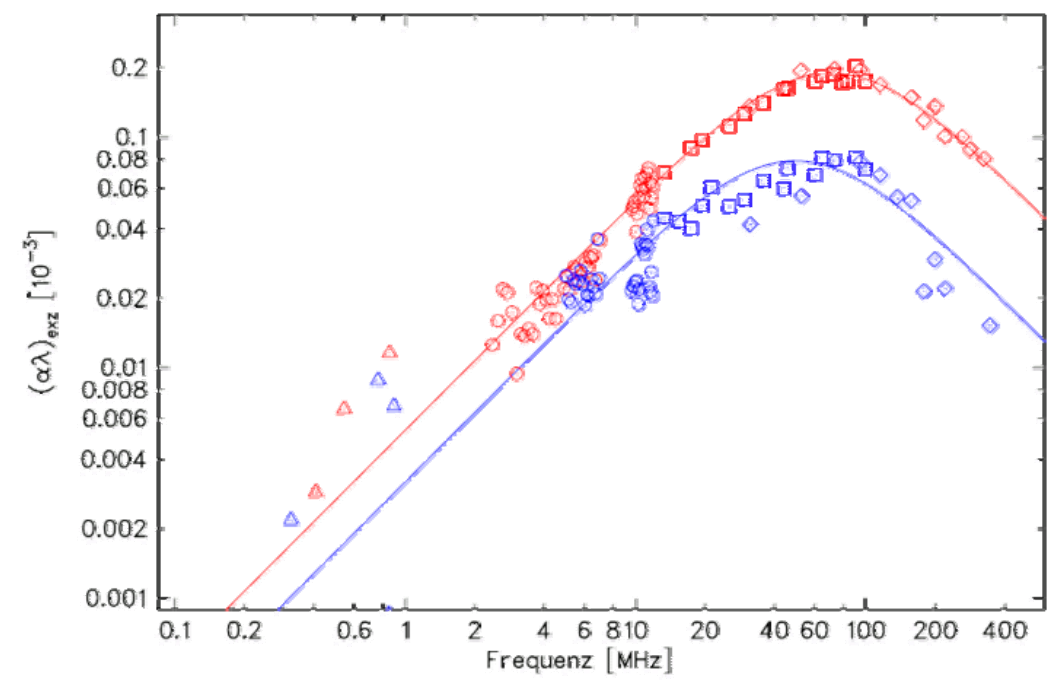

Abbildung 28: Ultraschallabsorptionsspektren von Glukose-Lösungen der Konzentrationen 100 mmol/L (blau) und 200 mmol/L (rot) in der $(\alpha \lambda)_{\text {exz }}$-Darstellung. Symbole wie in Abbildung 27. 
Die Parameter der Anpassungsrechnungen sind zusammen mit den Parametern aus [108] in Tabelle 4.1 aufgelistet. Da die einzige Debye-Spektralfunktion, die sich aus diesen Anpassungsrechnungen ergibt, eindeutig der teilweisen Rotation der $\mathrm{CH}_{2} \mathrm{OH}$-Gruppe um den Winkel $\omega$ zugeordnet werden kann, sind die beiden Parameter $A$ und $\tau$ bereits mit dem Index $\omega$ versehen, um eine möglichst einheitliche Notation bei der Darstellung der Spektren zu erreichen. Weiterhin ist in der letzten Spalte bereits

$$
\tilde{A} \equiv A \cdot \frac{R \cdot T}{\pi \cdot c_{s}^{2} \cdot \rho}=\Gamma \cdot\left(\Delta V_{S}\right)^{2}
$$

berechnet, indem die Konstanten $R, T$ und $\pi$ und die bekannten Größen $c_{S}$ und $\rho$ mittels Gl. (2.19) aus der Amplitude $A$ herausgerechnet wurden. Diese im Folgenden als Prozess-Amplitude $\tilde{\boldsymbol{A}}$ bezeichnete Größe ermöglicht für die unterschiedlichen betrachteten Systeme eine leichtere Vergleichbarkeit, da sie nur noch die prozessspezifischen Größen $\Gamma$ und $\Delta V_{S}$ beinhaltet.

\begin{tabular}{|c|c|c|c|c|c|c|}
\hline $\begin{array}{c}\text { Konzentration } \\
c[\mathrm{mmol} / \mathrm{L}]\end{array}$ & $\begin{array}{c}\text { Dichte } \\
\rho[\mathrm{g} / \mathrm{mL}]\end{array}$ & $\begin{array}{c}\text { Schallgeschw. } \\
c_{S}[\mathrm{~m} / \mathrm{s}]\end{array}$ & $\begin{array}{c}\text { Rel.-Zeit } \\
\tau_{\omega}[\mathrm{ns}]\end{array}$ & $\begin{array}{c}\text { Ampl. } \\
A_{\omega}\left[10^{-3}\right]\end{array}$ & $\begin{array}{l}\tilde{A}_{\omega}=\Gamma \cdot\left(\Delta V_{S}\right)^{2} \\
{\left[10^{-7} \cdot \mathrm{L} / \mathrm{mol}\right]}\end{array}$ & $\begin{array}{c}\text { B-Wert } \\
B \text { [ps] }\end{array}$ \\
\hline 100 & 1.004362 & 1503 & $3.2(3)$ & $0.16(2)$ & 0.56 & $32.9(1)$ \\
\hline 200 & 1.011069 & 1503 & $2.2(3)$ & $0.38(5)$ & 1.31 & $33.3(3)$ \\
\hline 300 & 1.018 & 1516 & 2.1 & 0.57 & 1.92 & $34.3(7)$ \\
\hline 500 & 1.031 & 1526 & 2.1 & 0.95 & 3.12 & $35.7(1)$ \\
\hline 800 & 1.051 & 1545 & 1.8 & 1.59 & 5.00 & $38.8(4)$ \\
\hline 1000 & 1.065 & 1563 & 2.0 & 1.99 & 6.04 & $40.3(9)$ \\
\hline
\end{tabular}

Tabelle 4.1: Parameter aus den Anpassungsrechnungen für die Ultraschallabsorptionsspektren von Glukose-Lösungen.

Die Messungen in [108] erfolgten über einen Frequenzbereich von etwa $200 \mathrm{kHz}$ bis $2 \mathrm{GHz}$. Während der Messungen zu der vorliegenden Arbeit stand die Hyperschallmessapparatur nicht zur Verfügung, die den Messbereich von etwa $500 \mathrm{MHz}$ bis $2 \mathrm{GHz}$ abdeckt. In diesem Frequenzbereich wird in [108] ein weiterer Absorptionsprozes der Glukose nachgewiesen, der einer Dimerisierung zugeschrieben wird. Da dieser Prozess in den Spektren dieser Arbeit nicht auftritt, ist der $B$-Wert zwischen den einzelnen Messungen nicht vergleichbar, da er in den Anpassungen in dieser Arbeit den zusätzlichen Prozess beinhaltet (siehe Gl. (2.21)), in [108] hingegen nicht. 


\subsection{Maltose}

Wie bei der Glukose wurden auch für Maltose-Lösungen die aus der Literatur [38] verfügbaren Spektren zu niedrigeren Konzentrationen erweitert. Die drei in dieser Arbeit gemessenen Spektren sind in Abbildung 29 bzw. Abbildung 30 wieder in $\operatorname{der}\left(\alpha / f^{2}\right)$ - und $\operatorname{der}(\alpha \lambda)_{\mathrm{exz}}$-Form dargestellt.

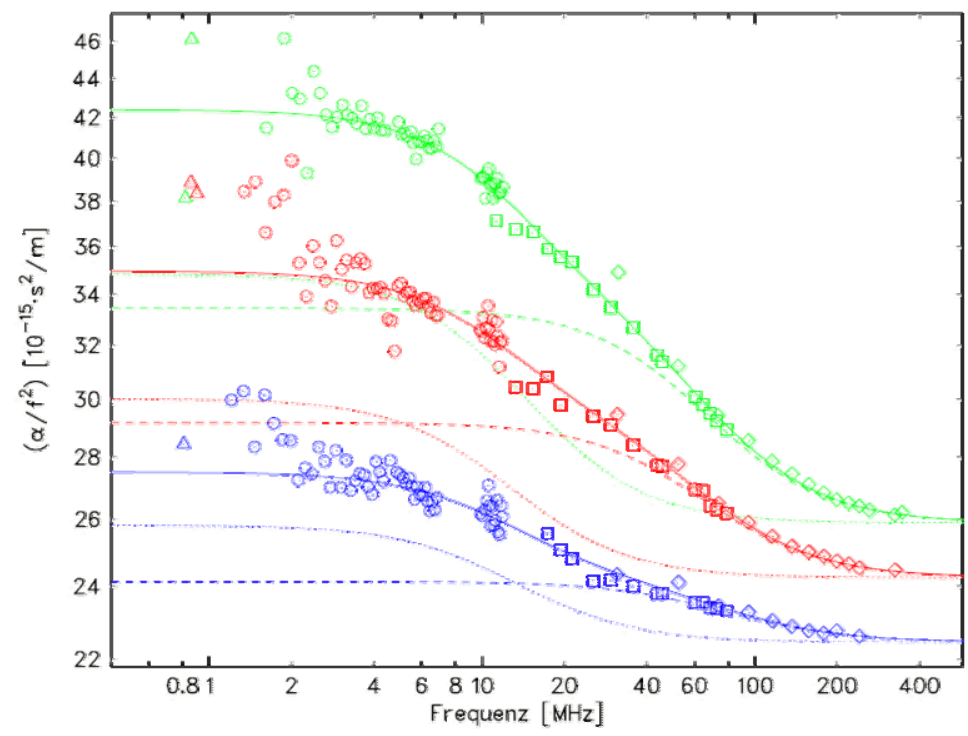

Abbildung 29: Ultraschallabsorptionsspektren von Maltose-Lösungen der Konzentrationen 100 $\mathrm{mmol} / \mathrm{L}$ (rot), $200 \mathrm{mmol} / \mathrm{L}$ (blau) und $300 \mathrm{mmol} / \mathrm{L}$ (grün) in der $\left(\alpha / f^{2}\right.$ )-Darstellung. Hier und im Folgenden: Die gestrichelten bzw. gepunkteten Linien stellen die einzelnen Debye-Spektralterme dar, die durchgezogenen Linien deren Summe. Symbole wie in Abbildung 27.

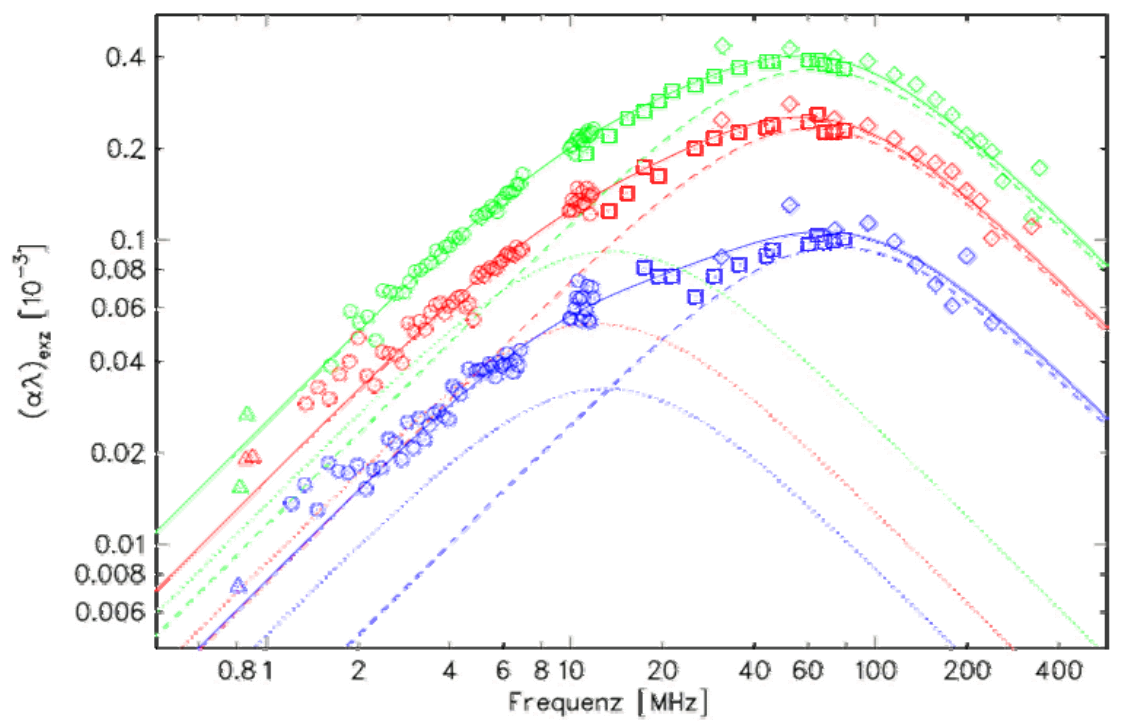

Abbildung 30: Ultraschallabsorptionsspektren von Maltose-Lösungen der Konzentrationen 100 $\mathrm{mmol} / \mathrm{L}$ (rot), $200 \mathrm{mmol} / \mathrm{L}$ (blau) und $300 \mathrm{mmol} / \mathrm{L}$ (grün) in der $(\alpha \lambda)_{\text {exz }}$-Darstellung. Symbole wie in Abbildung 27, Linien wie in Abbildung 29. 
4 Ergebnisse und Auswertung

\begin{tabular}{|c|c|c|c|c|c|c|c|c|c|c|}
\hline$c[\mathrm{mmol} / \mathrm{L}]$ & $\rho[\mathrm{g} / \mathrm{mL}]$ & $c_{S}$ & {$[\mathrm{~m} / \mathrm{s}]$} & $\tau_{\varphi \psi} \quad[\mathrm{ns}]$ & $A_{\varphi \psi} \quad[10$ & $\begin{array}{l}\tilde{A}_{\varphi \psi}\left[10^{-}\right. \\
\left.{ }^{7} \cdot \mathrm{L} / \mathrm{mol}\right]\end{array}$ & $\begin{array}{ll}\tau_{\omega} & {[\mathrm{ns}]}\end{array}$ & $A_{\omega}\left[10^{-3}\right]$ & $\begin{array}{l}\tilde{A}_{\omega} \\
{ }^{7} \cdot \mathrm{L} / \mathrm{mol}^{-}\end{array}$ & $B \quad[\mathrm{ps}]$ \\
\hline 100 & 1.009196 & & 1506 & $12.4(19)$ & $0.07(1)$ & 0.24 & 2.1(5) & $0.19(2)$ & 0.66 & $33.8(2)$ \\
\hline 200 & 1.023866 & & 1517 & $13.1(16)$ & $0.11(2)$ & 0.37 & $2.6(2)$ & $0.46(16)$ & 1.54 & $36.8(1)$ \\
\hline 300 & 1.037098 & & 1528 & $11.8(11)$ & $0.18(3)$ & 0.59 & $2.5(2)$ & $0.73(21)$ & 2.38 & $39.6(1)$ \\
\hline 500 & 1.063 & & 1551 & $6.8(2)$ & $0.78(4)$ & 2.41 & $1.5(1)$ & $1.27(4)$ & 3.92 & $41.4(5)$ \\
\hline 1000 & 1.129 & & 1614 & $9.1(2)$ & $1.53(5)$ & 4.10 & $1.7(1)$ & $3.39(7)$ & 9.10 & $61.2(3)$ \\
\hline 1500 & 1.193 & & 1687 & $11.6(4)$ & $3.31(1)$ & 7.69 & 1.1(1) & $9.7(5)$ & 22.54 & $101(2)$ \\
\hline 1800 & 1.232 & & 1734 & $19.0(6)$ & $3.32(11)$ & 7.07 & $1.9(1)$ & $12.4(3)$ & 26.41 & $146(1)$ \\
\hline
\end{tabular}

Tabelle 4.2: Parameter aus den Anpassungsrechnungen für die Ultraschallabsorptionsspektren von Maltose-Lösungen.

Neben dem schon bei der Glukose auftretenden Prozess der $\omega$-Rotation weisen die Ultraschallabsorptionsspektren der Maltose noch einen zweiten Prozess auf, der der (teilweisen) $\varphi$-/ $\psi$-Rotation zugeordnet wird. Die Parameter dieses Prozesses sind dementsprechend mit dem Index , $\varphi \psi$ ’ versehen.

Weiterhin ist wie bei der Glukose der $B$-Wert der Messungen aus dieser Arbeit nicht direkt mit den aufgeführten Werten aus [38] vergleichbar, da auch bei den Spektren der Maltose ein weiterer hochfrequenter (Dimerisierungs-)Prozess auftritt, der in dieser Arbeit nicht mit aufgenommen wurde. 


\subsection{Alkylglykoside}

\subsubsection{Alkylglukoside}

\subsubsection{1 n-Hexyl- $\beta$-D-Glukopyranosid $\left(C_{6} G_{1}\right)$}

Die beiden Spektren der untersuchten Lösungen von n-Hexyl- $\beta-D-G l u k o p y r a n o s i d ~\left(C_{6} \mathrm{G}_{1}\right)$ sind in Abbildung 31 und Abbildung 32 wieder in der $\left(\alpha / f^{2}\right)$ - und der $(\alpha \lambda)_{\text {exz }}$-Form dargestellt. In Tabelle 4.3 sind die Parameter der Spektren aus den Anpassungsrechnungen aufgelistet.

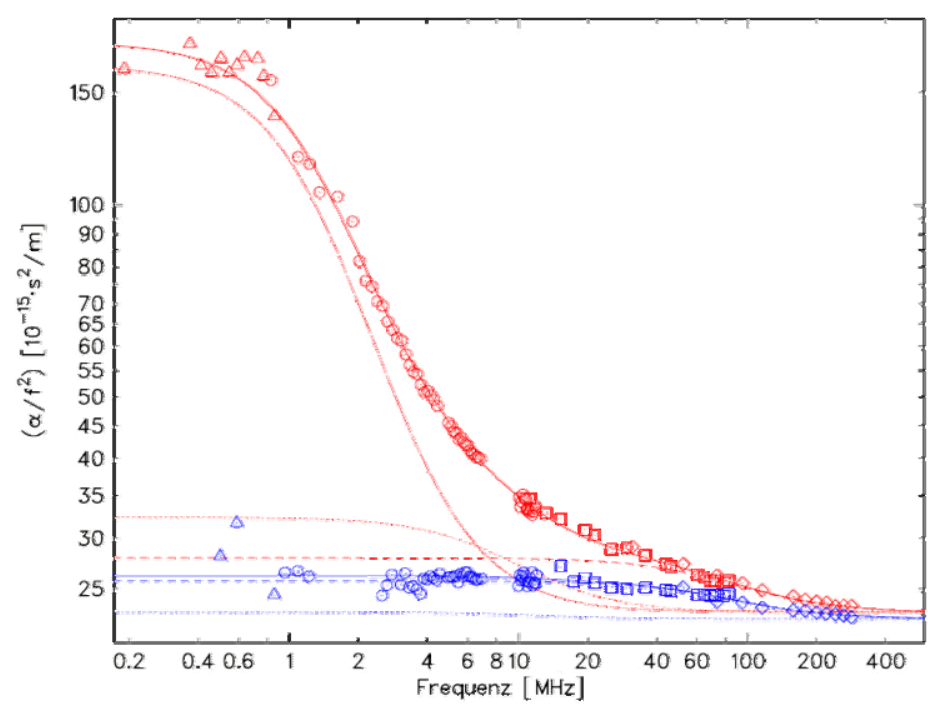

Abbildung 31: Ultraschallabsorptionsspektren von $C_{6} G_{1}$-Lösungen der Konzentrationen 150 $\mathrm{mmol} / \mathrm{L}$ (blau) und $200 \mathrm{mmol} / \mathrm{L}$ (rot) in der $\left(\alpha / f^{2}\right)$-Darstellung. Die einzelnen Debye-Spektralterme sind wieder durch gestrichelte bzw. gepunktete Linien angedeutet. Symbole wie in Abbildung 27.

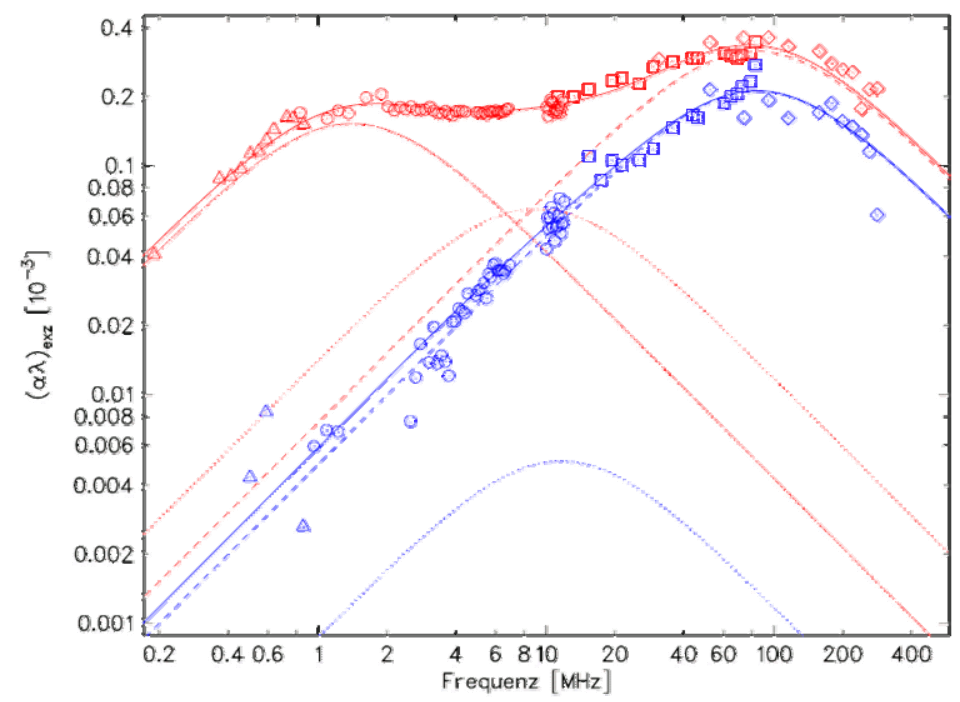

Abbildung 32: Ultraschallabsorptionsspektren von $C_{6} G_{1}$-Lösungen der Konzentrationen 150 mmol/L (blau) und 200 mmol/L (rot) in der $(\alpha \lambda)_{\text {exz }}$-Darstellung. Symbole wie in Abbildung 27. 
4 Ergebnisse und Auswertung

\begin{tabular}{r|c|c|r|r|r|r|r|r|r|r|r|r}
\hline \hline $\begin{array}{c}c \\
{[\mathrm{mmol} / \mathrm{L}]}\end{array}$ & \multicolumn{1}{c|}{$\begin{array}{c}\rho \\
{[\mathrm{g} / \mathrm{mL}]}\end{array}$} & $\begin{array}{c}c_{S} \\
{[\mathrm{~m} / \mathrm{s}]}\end{array}$ & $\begin{array}{c}\tau_{\text {mono }} \\
{[\mathrm{ns}]}\end{array}$ & $\begin{array}{c}A_{\text {mono }} \\
{\left[10^{-3}\right]}\end{array}$ & $\begin{array}{c}\tau_{\varphi \psi} \\
{[\mathrm{ns}]}\end{array}$ & $\begin{array}{c}A_{\varphi \psi} \\
{\left[10^{-3}\right]}\end{array}$ & $\begin{array}{c}\tilde{A}_{\varphi \psi} \\
{\left[10^{-7} \cdot \mathrm{L} / \mathrm{mol}\right]}\end{array}$ & $\begin{array}{c}\tau_{\omega} \\
{[\mathrm{ns}]}\end{array}$ & $\begin{array}{c}A_{\omega} \\
{\left[10^{-3}\right]}\end{array}$ & $\begin{array}{c}\tilde{A}_{\omega} \\
{\left[10^{-7} \cdot \mathrm{L} / \mathrm{mol}\right]}\end{array}$ & $\begin{array}{c}B \\
{[\mathrm{ps}]}\end{array}$ \\
\hline \hline 150 & -0.4 & 1.004854 & 1519 & - & - & 9 & 0.01 & 0.03 & 1.8 & 0.45 & 1.53 & 33.9 \\
200 & -0.2 & 1.007506 & 1527 & 114 & 0.305 & 18 & 0.13 & 0.44 & 1.9 & 0.63 & 2.12 & 35.1 \\
\hline \hline
\end{tabular}

Tabelle 4.3: Parameter aus den Anpassungsrechnungen für die Ultraschallabsorptionsspektren von $\mathrm{C}_{6} \mathrm{G}_{1}$-Lösungen.

In den beiden Abbildungen wird deutlich, dass der $\varphi-/ \psi$-Term bei $150 \mathrm{mmol} / \mathrm{L}$ nur extrem schwach auftritt. Eine Anpassung des Spektrums mit nur einer Debye-Spektralfunktion wäre in diesem Fall auch möglich gewesen, ohne dass die Abweichung der Messpunkte von der Spektralfunktion deutlich schlechter würde. Allerdings wurde aus Konsistenzgründen auch an dieses Spektrum eine zweite Debye-Spektralfunktion angepasst. Der Einfluss dieses zusätzlichen Terms auf die Parameter des $\omega$-Terms ist allerdings äußerst gering.

Weiterhin tritt bei $200 \mathrm{mmol} / \mathrm{L}$ bereits deutlich ein Term auf, der dem Monomeraustauschprozess zuzuordnen ist. Dies deutet entweder darauf hin, dass der Literaturwert der $\mathrm{cmc}$ von $250 \mathrm{mmol} / \mathrm{L}$ ([104]) zu hoch ist oder dass bereits deutlich unterhalb der $c m c$ eine erhebliche Anzahl von Mizellen vorliegt. 


\subsubsection{2 n-Heptyl- $\beta$-D-Glukopyranosid $\left(C_{7} G_{1}\right)$}

n-Heptyl- $\beta$-D-Glukopyranosid $\left(\mathrm{C}_{7} \mathrm{G}_{1}\right)$ weist eine $c m c$ in einer Größenordnung auf, die die Untersuchung sowohl unterhalb als auch oberhalb der $c m c$ erlaubt $(c m c \approx 70 \mathrm{mmol} / \mathrm{L})$. Daher wurde bei sieben verschiedenen Konzentrationen gemessen, von denen zwei unterhalb und vier oberhalb der $c m c$ liegen und eine bei der $c m c$ selbst liegt. Wegen der besseren Übersichtlichkeit sind in Abbildung 33 und Abbildung 34 jeweils nur drei der Spektren aufgetragen.

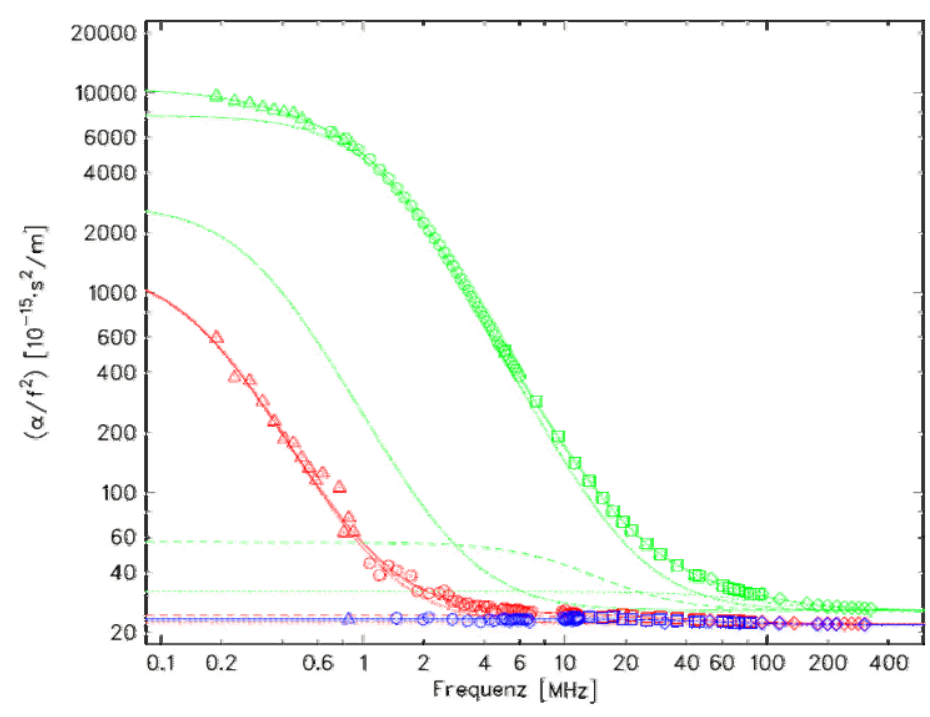

Abbildung 33: Ultraschallabsorptionsspektren von $\mathbf{C}_{7} \mathbf{G}_{1}$-Lösungen der Konzentrationen $50 \mathrm{mmol} / \mathrm{L}$ (blau), $75 \mathrm{mmol} / \mathrm{L}$ (rot) und $200 \mathrm{mmol} / \mathrm{L}$ (grün) in der $\left(\alpha / f^{2}\right)$-Darstellung. Die einzelnen DebyeSpektralterme sind wieder durch gestrichelte bzw. gepunktete Linien angedeutet. Symbole wie in Abbildung 27.

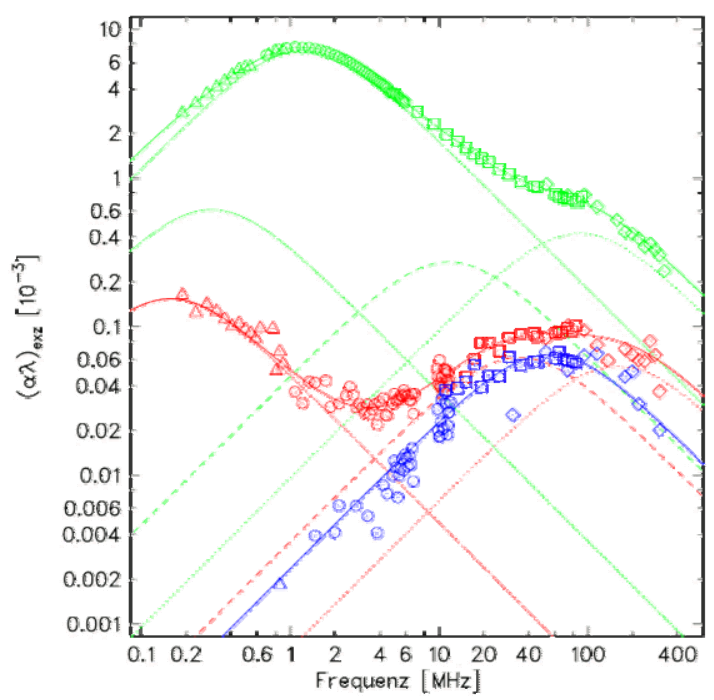

Abbildung 34: Ultraschallabsorptionsspektren von $\mathbf{C}_{7} \mathbf{G}_{1}$-Lösungen der Konzentrationen $50 \mathrm{mmol} / \mathrm{L}$ (blau), $75 \mathrm{mmol} / \mathrm{L}$ (rot) und $200 \mathrm{mmol} / \mathrm{L}$ (grün) in der $(\alpha \lambda)_{\text {exz }}$-Darstellung. Symbole wie in Abbildung 27. 
4 Ergebnisse und Auswertung

\begin{tabular}{|c|c|c|c|c|c|c|c|c|c|c|c|c|}
\hline $\begin{array}{c}c \\
{[\mathrm{mmol} / \mathrm{L}]}\end{array}$ & $X$ & $\begin{array}{c}\rho \\
{[\mathrm{g} / \mathrm{mL}]}\end{array}$ & $\begin{array}{c}c_{S} \\
{[\mathrm{~m} / \mathrm{s}]}\end{array}$ & $\begin{array}{l}\tau_{\text {mono }} \\
{[\mathrm{ns}]}\end{array}$ & $\begin{array}{l}A_{\text {mono }} \\
{\left[10^{-3}\right]}\end{array}$ & $\begin{array}{c}\tau_{\varphi \psi} \\
{[\mathrm{ns}]}\end{array}$ & $\begin{array}{c}A_{\varphi \psi} \\
{\left[10^{-3}\right]}\end{array}$ & $\begin{array}{c}\tilde{A}_{\varphi \psi} \\
{\left[10^{-7}\right.} \\
\cdot \mathrm{L} / \mathrm{mol}]\end{array}$ & $\begin{array}{c}\tau_{\omega} \\
{[\mathrm{ns}]}\end{array}$ & $\begin{array}{c}A_{\omega} \\
{\left[10^{-3}\right]}\end{array}$ & $\begin{array}{c}\tilde{A}_{\oplus} \\
{\left[10^{-7}\right.} \\
\cdot \mathrm{L} / \mathrm{mol}]\end{array}$ & $\begin{array}{c}B \\
{[\mathrm{ps}]}\end{array}$ \\
\hline 50 & -0.5 & 0.999720 & 1506 & - & - & - & - & - & $2.9(5)$ & $0.13(2)$ & 0.45 & $32.7(1)$ \\
\hline 60 & -0.24 & 1.000003 & 1506 & - & - & - & - & - & $2.2(3)$ & $0.13(2)$ & 0.45 & $32.8(1)$ \\
\hline 70 & -0.11 & 1.000540 & 1508 & - & - & $3.3(9)$ & $0.10(5)$ & 0.35 & $1^{*}$ & $0.10(1)$ & 0.35 & $32.8(2)$ \\
\hline 75 & -0.06 & 1.000643 & 1511 & $1013(77)$ & $0.31(2)$ & $4.6(8)$ & $0.12(3)$ & 0.41 & $1^{*}$ & $0.11(7)$ & 0.38 & $33.0(1)$ \\
\hline \multirow[t]{2}{*}{100} & 0.25 & 1.002000 & 1511 & $268(12)$ & $3.5(3)$ & $4.2(5)$ & $0.18(3)$ & 0.62 & $1^{*}$ & $0.23(6)$ & 0.79 & $33.6(1)$ \\
\hline & & & & $1200(138)$ & $4.2(2)$ & & & & & & & \\
\hline \multirow[t]{2}{*}{150} & 0.9 & 1.004143 & 1510 & $174(6)$ & $11.0(6)$ & $5.2(5)$ & $0.46(4)$ & 1.59 & $0.7(4)$ & $0.4(3)$ & 1.38 & $36.0(7)$ \\
\hline & & & & $763(16)$ & $3.6(4)$ & & & & & & & \\
\hline \multirow[t]{2}{*}{200} & 1.5 & 1.006200 & 1509 & $128(6)$ & $14.4(8)$ & $14(4)$ & $0.54(9)$ & 1.86 & $1.8(2)$ & $0.85(3)$ & 2.93 & $38.7(1)$ \\
\hline & & & & $538(38)$ & $1.2(7)$ & & & & & & & \\
\hline
\end{tabular}

Tabelle 4.4: Parameter aus den Anpassungsrechnungen für die Ultraschallabsorptionsspektren von $\mathbf{C}_{7} \mathbf{G}_{1}$-Lösungen.

Wie aus Tabelle 4.4 ersichtlich, tritt bei diesem System der Monomeraustauschprozess erst oberhalb von 70 mmol/L auf. Bei Konzentrationen von 100 mmol/L oder größer misslingt zudem die Anpassung der Spektren mit nur einem tieffrequenten Term, sondern es müssen zwei Debye-Spektralterme in diesem Frequenzbereich angepasst werden. Auf die Zuordnung dieses zusätzlichen Terms zu einem molekularen Prozess wird in Kapitel 5.1 eingegangen.

Weiterhin ist auffällig, dass bei den beiden niedrigsten untersuchten Konzentrationen der Prozess der $\varphi-/ \psi$ Rotation nicht auftaucht. Allerdings deuten sowohl die Relaxationszeit als auch die Amplitude des der $\omega$ Rotation zugeordneten Terms darauf hin, dass bei diesen beiden Konzentrationen die Amplituden der beiden unterschiedlichen Prozesse so klein sind, dass eine separate Auflösung unmöglich wird, so dass die in Tabelle 4.4 der $\omega$-Rotation zugeordneten Parameter eigentlich eine Überlagerung der beiden Prozesse beschreiben. 


\subsubsection{3 n-Octyl- $\beta$-D-Glukopyranosid $\left(C_{8} G_{1}\right)$}

n-Octyl- $\beta$-D-Glukopyranosid $\left(\mathrm{C}_{8} \mathrm{G}_{1}\right)$ ist von den betrachteten Systemen das in der Literatur am häufigsten untersuchte, da es insbesondere vielfach zur Solubilisierung von Membranproteinen eingesetzt wird. Daher wurden an diesem System Messungen bei sechs verschiedenen Konzentrationen durchgeführt. Wegen der besseren Übersichtlichkeit sind in Abbildung 35 bzw. Abbildung 36 wiederum jeweils nur drei dieser Spektren dargestellt.

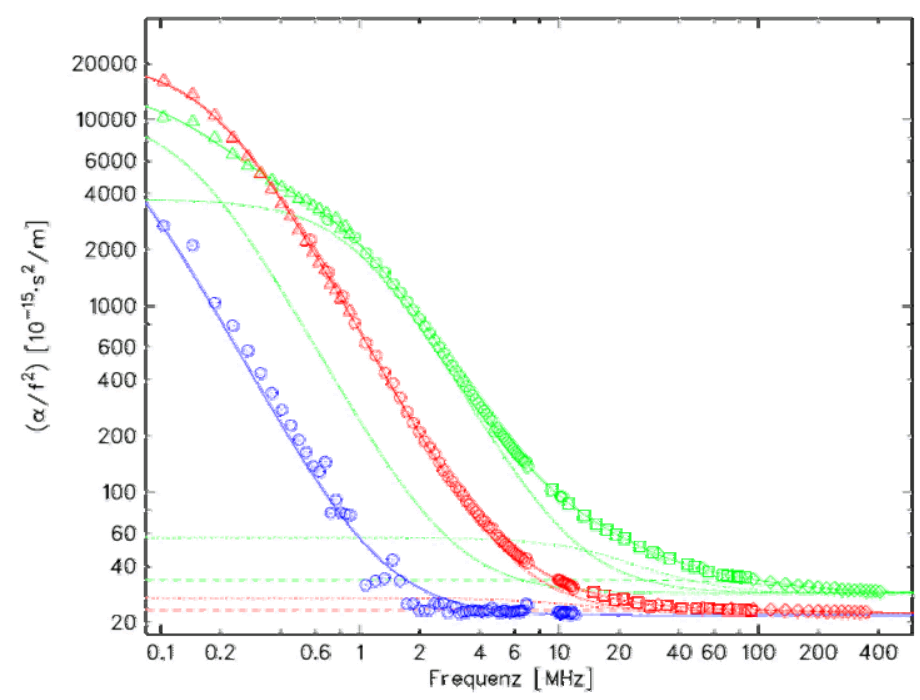

Abbildung 35: Ultraschallabsorptionsspektren von $\mathrm{C}_{8} \mathrm{G}_{1}$-Lösungen der Konzentrationen $25 \mathrm{mmol} / \mathrm{L}$ (blau), $75 \mathrm{mmol} / \mathrm{L}$ (rot) und $200 \mathrm{mmol} / \mathrm{L}$ (grün) in der $\left(\alpha / f^{2}\right)$-Darstellung. Die einzelnen DebyeSpektralterme sind wieder durch gestrichelte bzw. gepunktete Linien angedeutet. Symbole wie in Abbildung 27.

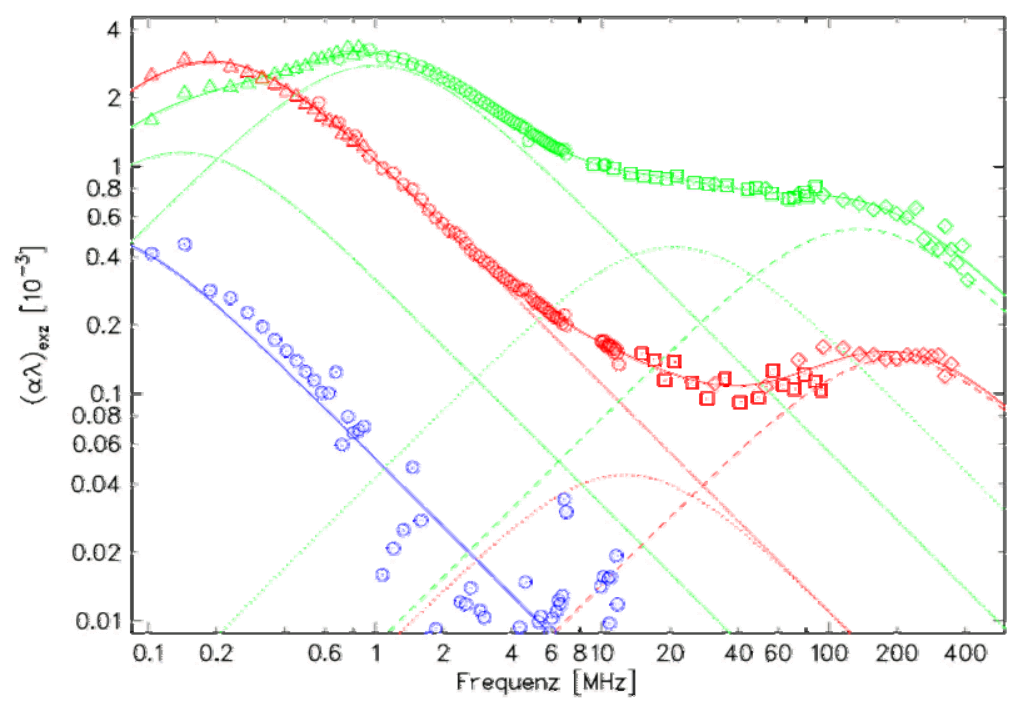

Abbildung 36: Ultraschallabsorptionsspektren von $\mathrm{C}_{8} \mathrm{G}_{1}$ - $\mathbf{L}$ ösungen der Konzentrationen $25 \mathrm{mmol} / \mathrm{L}$ (blau), $50 \mathrm{mmol} / \mathrm{L}$ (rot) und $200 \mathrm{mmol} / \mathrm{L}$ (grün) in der $(\alpha \lambda)_{\text {exz }}$-Darstellung. Symbole wie in Abbildung 27. 
4 Ergebnisse und Auswertung

\begin{tabular}{|c|c|c|c|c|c|c|c|c|c|c|c|c|}
\hline $\begin{array}{c}c \\
{[\mathrm{mmol} / \mathrm{L}]}\end{array}$ & $X$ & $\begin{array}{c}\rho \\
{[\mathrm{g} / \mathrm{mL}]}\end{array}$ & $\begin{array}{c}c_{S} \\
{[\mathrm{~m} / \mathrm{s}]}\end{array}$ & $\begin{array}{c}\tau_{\text {mono }} \\
{[\mathrm{ns}]}\end{array}$ & $\begin{array}{l}A_{\text {mono }} \\
{\left[10^{-3}\right]}\end{array}$ & $\begin{array}{c}\tau_{\varphi \psi} \\
{[\mathrm{ns}]}\end{array}$ & $\begin{array}{c}A_{\varphi \psi} \\
{\left[10^{-3}\right]}\end{array}$ & $\begin{array}{c}\tilde{A}_{\varphi \psi} \\
{\left[10^{-7}\right.} \\
\cdot \mathrm{L} / \mathrm{mol}]\end{array}$ & $\begin{array}{c}\tau_{\omega} \\
{[\mathrm{ns}]}\end{array}$ & $\begin{array}{c}A_{\omega} \\
{\left[10^{-3}\right]}\end{array}$ & $\begin{array}{c}\tilde{A}_{\omega} \\
{\left[10^{-7}\right.} \\
\cdot \mathrm{L} / \mathrm{mol}]\end{array}$ & $\begin{array}{c}B \\
{[\mathrm{ps}]}\end{array}$ \\
\hline 25 & 0 & 0.998192 & 1502 & $3066(145)$ & $1 *$ & - & - & - & - & - & - & $32.8(3)$ \\
\hline 30 & 0.2 & 0.998425 & 1502 & $1737(17)$ & $1.94(17)$ & - & - & - & $2 *$ & $0.10(2)$ & 0.35 & $32.7(2)$ \\
\hline 50 & 1 & 0.999142 & 1502 & $839(14)$ & $5.78(8)$ & $12.6(1)$ & $0.09(1)$ & 0.32 & $0.8(3)$ & $0.28(13)$ & 0.98 & $33.6(3)$ \\
\hline 75 & 2 & 0.999918 & 1503 & $582(18)$ & $6.62(14)$ & $16(21)$ & $0.02(2)$ & 0.07 & $1.8(2)$ & $0.57(4)$ & 1.99 & $35.7(2)$ \\
\hline 100 & 3 & 1.001147 & 1500 & $358(5)$ & $6.47(6)$ & $6.7(6)$ & $0.37(3)$ & 1.30 & $1.0(4)$ & $0.39(14)$ & 1.37 & $36.6(4)$ \\
\hline 200 & 7 & 1.005238 & 1500 & $\begin{array}{r}159(3) \\
1131(124)\end{array}$ & $\begin{array}{l}5.56(12) \\
2.29(11)\end{array}$ & 7.7(4) & $0.88(3)$ & 3.07 & $1.2(1)$ & $1.06(5)$ & 3.70 & $42.9(2)$ \\
\hline
\end{tabular}

Tabelle 4.5: Parameter aus den Anpassungsrechnungen für die Ultraschallabsorptionsspektren von $\mathrm{C}_{8} \mathrm{G}_{1}$-Lösungen.

Wie schon bei den Messungen an $\mathrm{C}_{7} \mathrm{G}_{1}$-Lösungen erweist es sich auch hier als schwierig, die beiden hochfrequenten Prozesse bei niedrigen Konzentrationen separat aufzulösen, so dass auch hier davon auszugehen ist, dass die Parameter der 30 mmol/L-Lösung vermutlich eine Überlagerung der beiden Prozesse beschreiben. Bei der 25 mmol/L-Lösung sind die Amplituden bereits so klein, dass eine sinnvolle Anpassung von Relaxationstermen überhaupt nicht mehr möglich ist. Der im Vergleich zur $30 \mathrm{mmol} / \mathrm{L}$-Lösung größere statt kleinere $B$-Wert resultiert dementsprechend daher, dass er offenbar diese beiden Prozesse beinhaltet.

Der Monomeraustauschprozess liegt insbesondere bei der $25 \mathrm{mmol} / \mathrm{L}$-Lösung so tieffrequent, dass sein Maximum deutlich außerhalb der Messbereiches liegt. Daher musste bei der Anpassung dieses Spektrums die Amplitude des entsprechenden Relaxationsterms festgehalten werden.

Im Falle der Messung bei der höchsten Tensidkonzentration ( $200 \mathrm{mmol} / \mathrm{L})$ muss für eine adäquate Beschreibung des tieffrequenten Teils des Spektrums - wie schon bei dem System $\mathrm{C}_{7} \mathrm{G}_{1}$ - ein weiterer Spektralterm hinzugenommen werden, auf den ebenfalls in Kapitel 5.1 eingegangen wird.

Weiterhin wurden für dieses System bei drei Konzentrationen $(25 \mathrm{mmol} / \mathrm{L}, 50 \mathrm{mmol} / \mathrm{L}$ und $75 \mathrm{mmol} / \mathrm{L}$ ) Messungen bei weiteren Temperaturen durchgeführt, um einen Einblick in die Temperaturabhängigkeit der Parameter der einzelnen Prozesse erhalten zu können. Die resultierenden Parameter dieser Spektren sind in Tabelle 4.6 aufgelistet. 
4 Ergebnisse und Auswertung

\begin{tabular}{|c|c|c|c|c|c|c|c|c|c|c|c|c|}
\hline $\begin{array}{c}T \\
{\left[{ }^{\circ} \mathrm{C}\right]}\end{array}$ & $X$ & $\begin{array}{c}\rho \\
{[\mathrm{g} / \mathrm{mL}]}\end{array}$ & $\begin{array}{c}c_{S} \\
{[\mathrm{~m} / \mathrm{s}]}\end{array}$ & $\begin{array}{l}\tau_{\text {mono }} \\
{[\mathrm{ns}]}\end{array}$ & $\begin{array}{l}A_{\text {mono }} \\
{\left[10^{-3}\right]}\end{array}$ & $\begin{array}{c}\tau_{\varphi \psi} \\
{[\mathrm{ns}]}\end{array}$ & $\begin{array}{c}A_{\varphi \psi} \\
{\left[10^{-3}\right]}\end{array}$ & $\begin{array}{c}\tilde{A}_{\varphi \psi} \\
{\left[10^{-7}\right.} \\
\cdot \mathrm{L} / \mathrm{mol}]\end{array}$ & $\begin{array}{c}\tau_{\omega} \\
{[\mathrm{ns}]}\end{array}$ & $\begin{array}{c}A_{\omega} \\
{\left[10^{-3}\right]}\end{array}$ & $\begin{array}{c}\tilde{A}_{\omega} \\
{\left[10^{-7}\right.} \\
\cdot \mathrm{L} / \mathrm{mol}]\end{array}$ & $\begin{array}{c}B \\
{[\mathrm{ps}]}\end{array}$ \\
\hline
\end{tabular}

$\mathrm{c}=25 \mathrm{mmol} / \mathrm{L}$

\begin{tabular}{|c|c|c|c|c|c|c|c|c|c|c|c|c|}
\hline 15 & -0.1 & 1.000343 & 1473 & - & - & - & - & - & - & - & - & $43.9(4)$ \\
\hline 25 & 0 & 0.998192 & 1502 & $3066(145)$ & $1^{*}$ & - & - & - & - & - & - & $32.8(3)$ \\
\hline 30 & 0.1 & 0.996737 & 1514 & $1489(54)$ & $1 *$ & - & - & - & - & - & - & $28.9(3)$ \\
\hline
\end{tabular}

$\mathrm{c}=50 \mathrm{mmol} / \mathrm{L}$

\begin{tabular}{|c|c|c|c|c|c|c|c|c|c|c|c|c|}
\hline 15 & 0.8 & 1.001385 & 1473 & $1686(64)$ & $7.75(24)$ & $59(22)$ & $0.04(1)$ & 0.14 & $2.1(1)$ & $0.31(2)$ & 1.13 & $46.8(1)$ \\
\hline 25 & 1 & 0.999142 & 1502 & $839(14)$ & $5.78(8)$ & $12.6(1)$ & $0.09(1)$ & 0.32 & $0.8(3)$ & $0.28(13)$ & 0.98 & $33.6(3)$ \\
\hline 30 & 1.2 & 0.997671 & 1514 & $662(18)$ & $5.58(13)$ & - & - & - & $1.6(2)$ & $0.40(4)$ & 1.40 & $30.7(2)$ \\
\hline
\end{tabular}

$\mathrm{c}=75 \mathrm{mmol} / \mathrm{L}$

\begin{tabular}{|c|c|c|c|c|c|c|c|c|c|c|c|c|}
\hline 10 & 1.4 & 1.002961 & 1458 & $1288(104)$ & $9.8(7)$ & $3.9(12)$ & $0.30(18)$ & 1.05 & $1.0(9)$ & $0.28(13)$ & 0.98 & $56.7(3)$ \\
\hline 15 & 1.7 & 1.002208 & 1475 & $869(27)$ & $7.72(20)$ & $6.8(15)$ & $0.15(3)$ & 0.53 & $1 *$ & $0.34(7)$ & 1.19 & $48.4(1)$ \\
\hline 20 & 1.8 & 1.001186 & 1487 & $692(2)$ & $6.96(17)$ & $3(4)$ & $0.1(8)$ & 0.35 & $2.0(1)$ & $0.5(8)$ & 1.75 & $41.3(1)$ \\
\hline 25 & 2 & 0.999918 & 1503 & $582(18)$ & $6.62(14)$ & $16(21)$ & $0.02(2)$ & 0.07 & $1.8(2)$ & $0.57(4)$ & 1.99 & $35.7(2)$ \\
\hline 30 & 2.3 & 0.998424 & 1513 & $442(7)$ & $5.78(8)$ & - & - & & $2.1(1)$ & $0.54(1)$ & 1.90 & $31.7(1)$ \\
\hline
\end{tabular}

Tabelle 4.6: Parameter aus den Anpassungsrechnungen für die Ultraschallabsorptionsspektren von $\mathrm{C}_{8} \mathbf{G}_{1}$-Lösungen dreier Konzentrationen bei verschiedenen Temperaturen. Die temperaturabhängigen reduzierten Konzentrationen wurden mit Literaturwerten für die temperaturabhängige $\mathrm{cmc}$ berechnet: $10{ }^{\circ} \mathrm{C}: c m c=31.5 \mathrm{mmol} / \mathrm{L} ; 15^{\circ} \mathrm{C}: c m c=28 \mathrm{mmol} / \mathrm{L} ; 2{ }^{\circ} \mathrm{C}: c m c=27 \mathrm{mmol} / \mathrm{L} ; 25^{\circ} \mathrm{C}: c m c=25 \mathrm{mmol} / \mathrm{L} ; 30$ ${ }^{\circ} \mathrm{C}: \mathrm{cmc}=23 \mathrm{mmol} / \mathrm{L}[115]$. 


\subsubsection{4 n-Nonyl- $\beta$-D-Glukopyranosid $\left(C_{9} G_{1}\right)$}

Am System n-Nonyl- $\beta$-D-Glukopyranosid $\left(\mathrm{C}_{9} \mathrm{G}_{1}\right)$ /Wasser wurden Messungen bei vier verschiedenen Konzentrationen durchgeführt. Die Spektren sind in Abbildung 37 bzw. Abbildung 38 dargestellt.

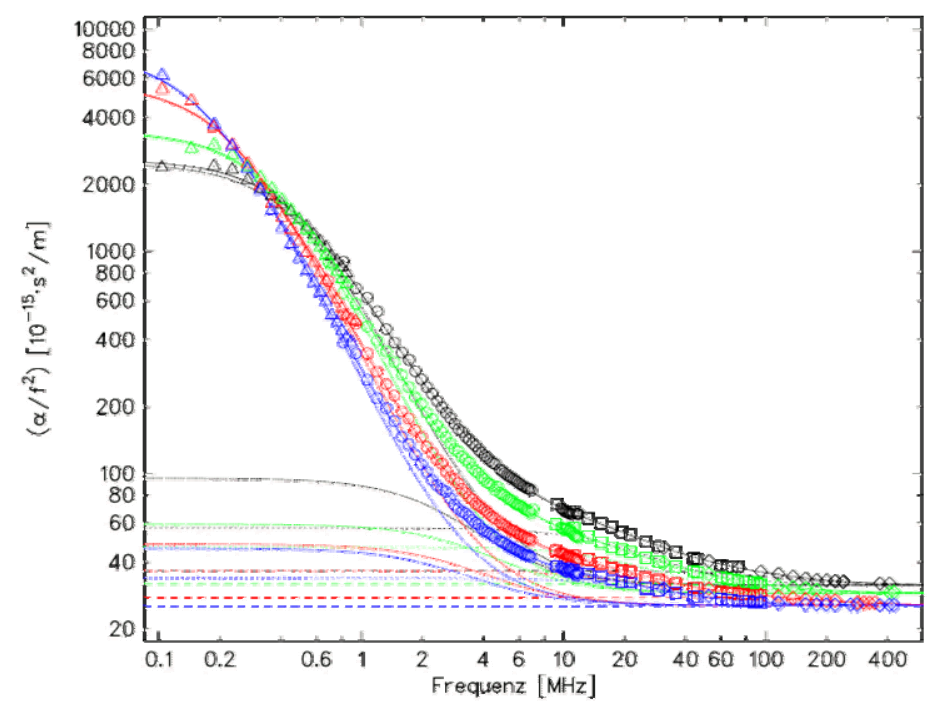

Abbildung 37: Ultraschallabsorptionsspektren von $\mathbf{C}_{9} \mathbf{G}_{1}$-Lösungen der Konzentrationen 75 mmol/L (blau), $100 \mathrm{mmol} / \mathrm{L}$ (rot), $150 \mathrm{mmol} / \mathrm{L}$ (grün) und $200 \mathrm{mmol} / \mathrm{L}$ (schwarz) in der $\left(\alpha / f^{2}\right)$-Darstellung. Die einzelnen Debye-Spektralterme sind wieder durch gestrichelte bzw. gepunktete Linien angedeutet. Symbole wie in Abbildung 27.

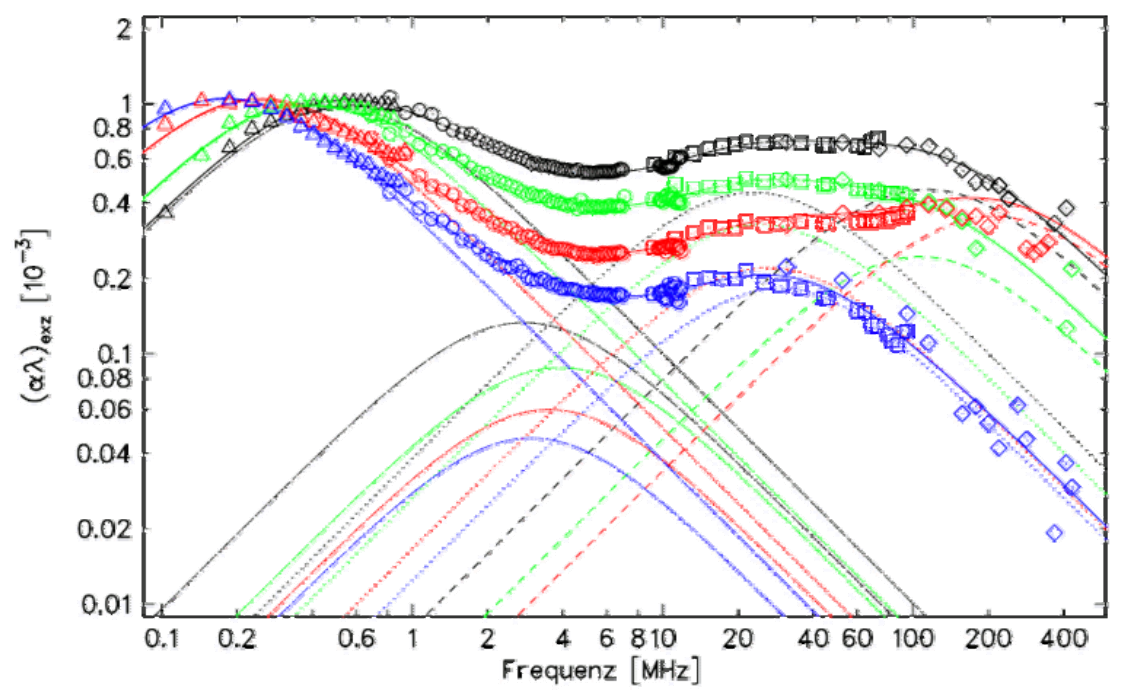

Abbildung 38: Ultraschallabsorptionsspektren von $\mathrm{C}_{9} \mathrm{G}_{1}$-Lösungen der Konzentrationen $75 \mathrm{mmol} / \mathrm{L}$ (blau), $100 \mathrm{mmol} / \mathrm{L}$ (rot), $150 \mathrm{mmol} / \mathrm{L}$ (grün) und $200 \mathrm{mmol} / \mathrm{L}$ (schwarz) in der $(\alpha \lambda)_{\mathrm{exz}}$-Darstellung. Die Amplitude des hochfrequenten Terms ist bei $75 \mathrm{mmol} / \mathrm{L}$ so klein, dass der Term in dieser Auftragung nicht sichtbar ist. Symbole wie in Abbildung 27. 
4 Ergebnisse und Auswertung

\begin{tabular}{|c|c|c|c|c|c|c|c|c|c|c|c|c|}
\hline $\begin{array}{c}c \\
{[\mathrm{mmol} / \mathrm{L}]}\end{array}$ & $X$ & $\begin{array}{c}\rho \\
{[\mathrm{g} / \mathrm{mL}]}\end{array}$ & $\begin{array}{c}c_{S} \\
{[\mathrm{~m} / \mathrm{s}]}\end{array}$ & $\begin{array}{c}\tau_{\text {mono }} \\
{[\mathrm{ns}]}\end{array}$ & $\begin{array}{l}A_{\text {mono }} \\
{\left[10^{-3}\right]}\end{array}$ & $\begin{array}{c}\tau_{\varphi \psi} \\
{[\mathrm{ns}]}\end{array}$ & $\begin{array}{c}A_{\varphi \psi} \\
{\left[10^{-3}\right]}\end{array}$ & $\begin{array}{c}\tilde{A}_{\varphi \psi} \\
{\left[10^{-7}\right.} \\
\cdot \mathrm{L} / \mathrm{mol}]\end{array}$ & $\begin{array}{c}\tau_{\oplus} \\
{[\mathrm{ns}]}\end{array}$ & $\begin{array}{c}A_{\omega} \\
{\left[10^{-3}\right]}\end{array}$ & $\begin{array}{c}\tilde{A}_{\omega} \\
{\left[10^{-7}\right.} \\
\cdot \mathrm{L} / \mathrm{mol}]\end{array}$ & $\begin{array}{c}B \\
{[\mathrm{ps}]}\end{array}$ \\
\hline 75 & 9.7 & 0.999642 & 1499 & $\begin{array}{r}887(24) \\
53(8)\end{array}$ & $\begin{array}{l}2.08(4) \\
0.10(1)\end{array}$ & 5.4(3) & $0.37(2)$ & 1.30 & $1^{*}$ & $0.01(4)$ & 0.04 & $38.2(1)$ \\
\hline 100 & 13.3 & 1.000538 & 1499 & $\begin{array}{r}647(14) \\
45^{*}\end{array}$ & $\begin{array}{l}2.06(3) \\
0.12(1)\end{array}$ & $6.0(4)$ & $0.44(3)$ & 1.54 & $0.8(2)$ & $0.71(19)$ & 2.49 & $38.1(4)$ \\
\hline 150 & 20.4 & 1.002341 & 1499 & $\begin{array}{r}402(9) \\
36(7)\end{array}$ & $\begin{array}{l}2.00(2) \\
0.18(2)\end{array}$ & $5.9(7)$ & $0.71(8)$ & 2.49 & $1.0(11)$ & $0.6(10)$ & 2.10 & $43.0(3)$ \\
\hline 200 & 27.6 & 1.004130 & 1499 & $\begin{array}{l}302(9) \\
56(11)\end{array}$ & $\begin{array}{r}1.93(4) \\
0.2(4)\end{array}$ & $7.0(5)$ & $0.86(6)$ & 3.01 & $1.5(2)$ & $0.87(5)$ & 3.04 & $47.1(2)$ \\
\hline
\end{tabular}

Tabelle 4.7: Parameter aus den Anpassungsrechnungen für die Ultraschallabsorptionsspektren von $\mathrm{C}_{9} \mathrm{G}_{1}$-Lösungen.

Alle vier untersuchten Spektren dieses Systems weisen neben den Termen der $\omega$-Rotation und der $\varphi$-/ $\psi$-Rotation sowie einem Term für den Monomeraustauschprozess noch einen weiteren auf. Auch auf diesen wird in Kapitel 5.1 näher eingegangen. 


\subsubsection{Alkylmaltoside}

\subsubsection{1 n-Hexyl- $\beta-D$-Maltopyranosid $\left(C_{6} G_{2}\right)$}

Am System n-Hexyl- $\beta$-D-Maltopyranosid $\left(\mathrm{C}_{6} \mathrm{G}_{2}\right)$ /Wasser wurden Messungen bei sieben verschiedenen Konzentrationen durchgeführt. Die Spektren der Lösungen dreier Konzentrationen sind in Abbildung 39 bzw. Abbildung 40 dargestellt.

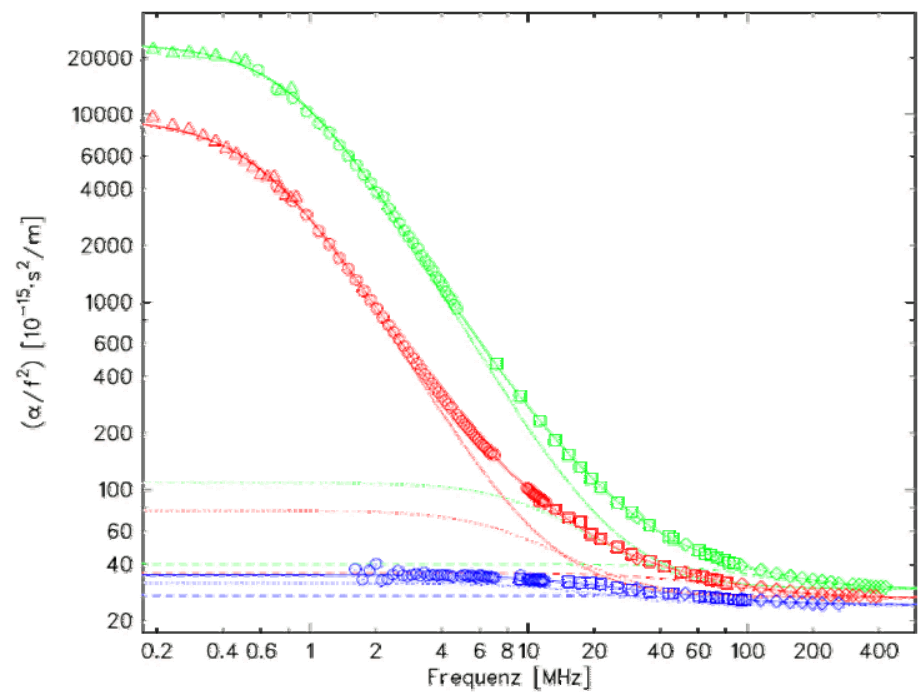

Abbildung 39: Ultraschallabsorptionsspektren von $\mathbf{C}_{6} \mathbf{G}_{2}$-Lösungen der Konzentrationen 150 $\mathrm{mmol} / \mathrm{L}$ (blau), $225 \mathrm{mmol} / \mathrm{L}$ (rot) und $300 \mathrm{mmol} / \mathrm{L}$ (grün) in der $\left(\alpha / f^{2}\right)$-Darstellung. Die einzelnen Debye-Spektralterme sind wieder durch gestrichelte bzw. gepunktete Linien angedeutet. Symbole wie in Abbildung 27.

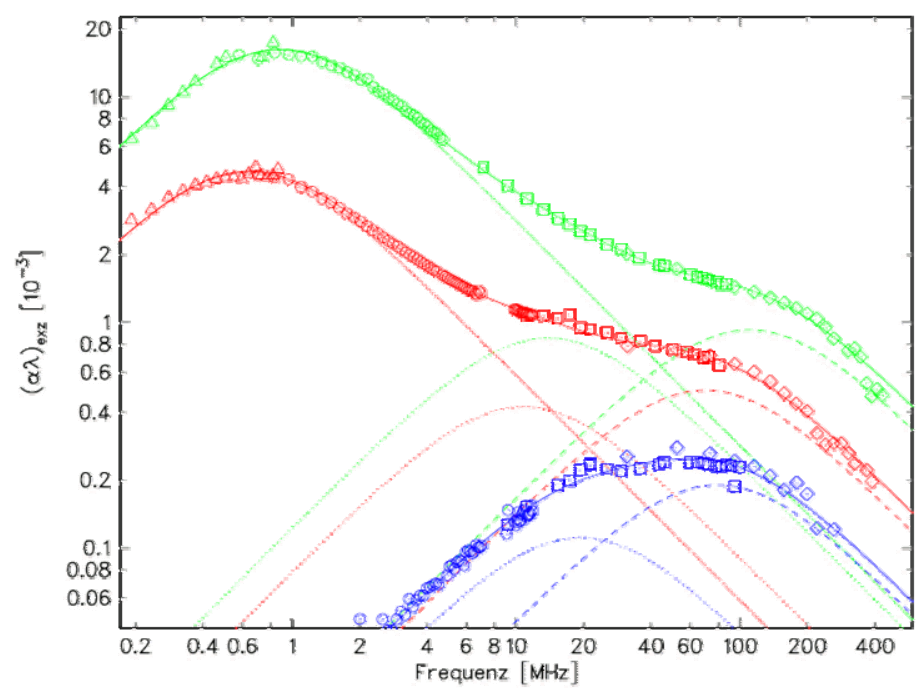

Abbildung 40: Ultraschallabsorptionsspektren von $\mathbf{C}_{6} \mathbf{G}_{2}$-Lösungen der Konzentrationen 150 $\mathrm{mmol} / \mathrm{L}$ (blau), $225 \mathrm{mmol} / \mathrm{L}$ (rot) und $300 \mathrm{mmol} / \mathrm{L}$ (grün) in der $(\alpha \lambda)_{\text {exz }}$-Darstellung. Symbole wie in Abbildung 27. 
4 Ergebnisse und Auswertung

\begin{tabular}{|c|c|c|c|c|c|c|c|c|c|c|c|c|}
\hline $\begin{array}{c}c \\
{[\mathrm{mmol} / \mathrm{L}]}\end{array}$ & $X$ & $\begin{array}{c}\rho \\
{[\mathrm{g} / \mathrm{mL}]}\end{array}$ & $\begin{array}{c}c_{S} \\
{[\mathrm{~m} / \mathrm{s}]}\end{array}$ & $\begin{array}{l}\tau_{\text {mono }} \\
{[\mathrm{ns}]}\end{array}$ & $\begin{array}{l}A_{\text {mono }} \\
{\left[10^{-3}\right]}\end{array}$ & $\begin{array}{c}\tau_{\varphi \psi} \\
{[\mathrm{ns}]}\end{array}$ & $\begin{array}{c}A_{\varphi \psi} \\
{\left[10^{-3}\right]}\end{array}$ & $\begin{array}{c}\tilde{A}_{\varphi \psi} \\
{\left[10^{-7}\right.} \\
\cdot \mathrm{L} / \mathrm{mol}]\end{array}$ & $\begin{array}{c}\tau_{\omega} \\
{[\mathrm{ns}]}\end{array}$ & $\begin{array}{c}A_{\omega} \\
{\left[10^{-3}\right]}\end{array}$ & $\begin{array}{c}\tilde{A}_{\omega} \\
{\left[10^{-7}\right.} \\
\cdot \mathrm{L} / \mathrm{mol}]\end{array}$ & $\begin{array}{c}B \\
{[\mathrm{ps}]}\end{array}$ \\
\hline 150 & -0.29 & 1.014358 & 1524 & - & - & $8.5(10)$ & $0.22(3)$ & 0.74 & $2^{*}$ & $0.38(5)$ & 1.27 & $37.1(2)$ \\
\hline 175 & -0.17 & 1.019090 & 1529 & $90(5)$ & $0.28(1)$ & $6.5(9)$ & $0.48(7)$ & 1.59 & $2^{*}$ & $0.39(1)$ & 1.29 & $38.6(2)$ \\
\hline 200 & -0.05 & 1.020203 & 1532 & $123(6)$ & $1.63(6)$ & $12.8(11)$ & $0.47(2)$ & 1.55 & $2^{*}$ & $0.71(4)$ & 2.34 & $39.7(1)$ \\
\hline & & & & $1008(531)$ & $0.39(6)$ & & & & & & & \\
\hline 225 & 0.07 & 1.023725 & 1538 & $250(3)$ & $9.21(7)$ & $14.8(7)$ & $0.85(2)$ & 2.77 & $2.3(1)$ & $1.00(2)$ & 3.26 & $40.9(1)$ \\
\hline 250 & 0.19 & 1.026214 & 1539 & $227(4)$ & 19.1(3) & $12 *$ & $1.1(1)$ & 3.57 & $1.9(2)$ & $1.23(6)$ & 3.99 & $42.7(2)$ \\
\hline 275 & 0.31 & 1.028817 & 1535 & 209(5) & $26.6(4)$ & $12 *$ & $1.41(11)$ & 4.59 & $1.6(2)$ & $1.33(9)$ & 4.33 & $45.0(4)$ \\
\hline 300 & 0.43 & 1.034480 & 1535 & 181(3) & $32.2(4)$ & $11.5(14)$ & $1.71(11)$ & 5.54 & $1.9(1)$ & $1.44(10)$ & 4.66 & $45.2(2)$ \\
\hline
\end{tabular}

Tabelle 4.8: Parameter aus den Anpassungsrechnungen für die Ultraschallabsorptionsspektren von $\mathrm{C}_{6} \mathbf{G}_{2}$-Lösungen.

Bei allen untersuchten Konzentrationen dieses Systems treten im Ultraschallspektrum Terme für die $\omega$-Rotation, die $\varphi$-/ $\psi$-Rotation und den Monomeraustauschprozess auf. Lediglich bei $150 \mathrm{mmol} / \mathrm{L}$ ist letzterer nicht nachweisbar und bei 200 mmol/L tritt wieder ein zusätzlicher Term im tieffrequenten Bereich auf (siehe Kapitel 5.1). 


\subsubsection{2 n-Octyl- $\beta$-D-Maltopyranosid $\left(C_{8} G_{2}\right)$}

Am System n-Octyl- $\beta$-D-Maltopyranosid $\left(\mathrm{C}_{8} \mathrm{G}_{2}\right)$ /Wasser wurden Messungen bei drei verschiedenen Konzentrationen durchgeführt. Die resultierenden Spektren sind in Abbildung 41 bzw. Abbildung 42 dargestellt.

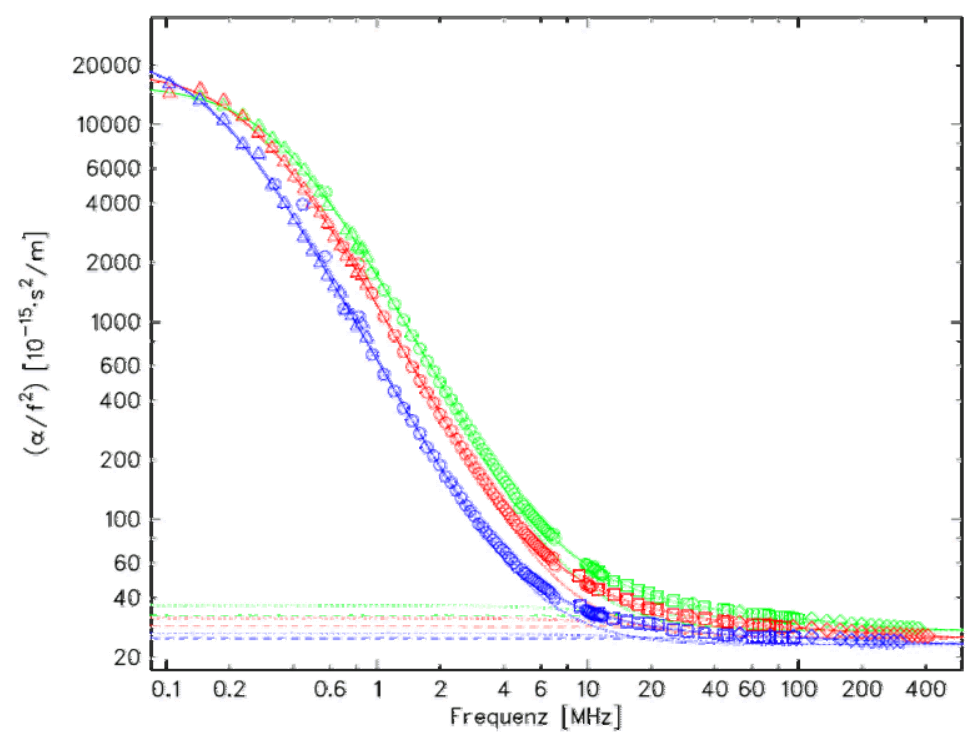

Abbildung 41: Ultraschallabsorptionsspektren von $\mathrm{C}_{8} \mathrm{G}_{2}$-Lösungen der Konzentrationen $50 \mathrm{mmol} / \mathrm{L}$ (blau), $75 \mathrm{mmol} / \mathrm{L}$ (rot) und $100 \mathrm{mmol} / \mathrm{L}$ (grün) in der $\left(\alpha / f^{2}\right)$-Darstellung. Die einzelnen DebyeSpektralterme sind wieder durch gestrichelte bzw. gepunktete Linien angedeutet. Symbole wie in Abbildung 27.

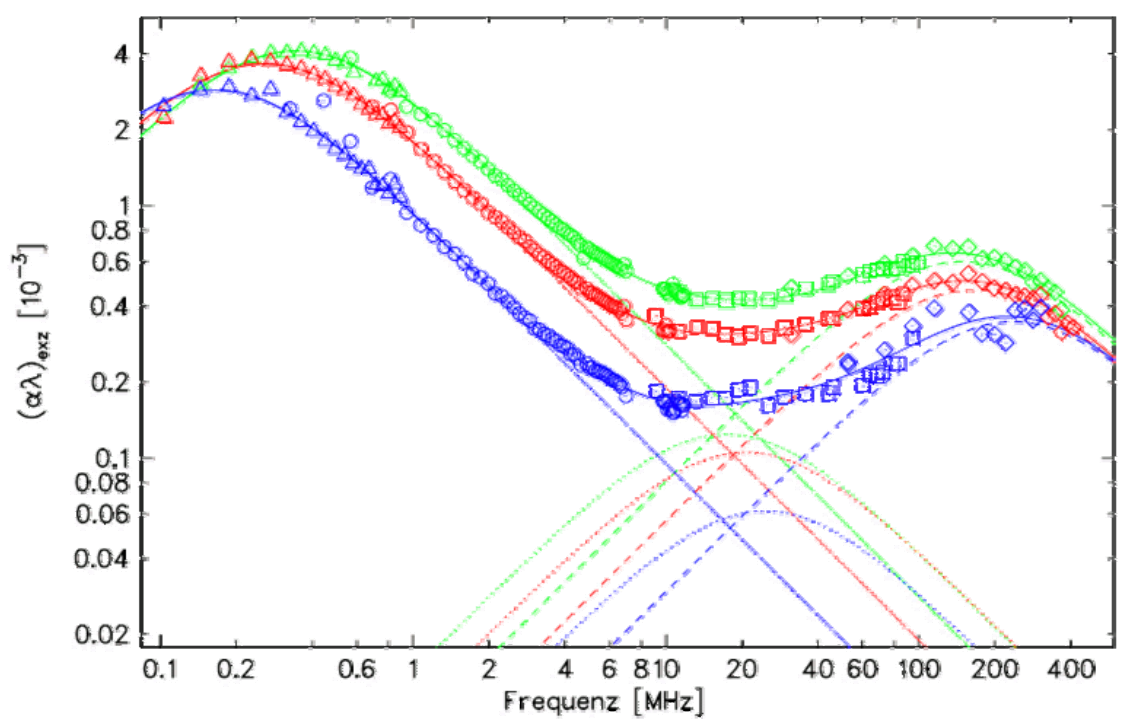

Abbildung 42: Ultraschallabsorptionsspektren von $\mathrm{C}_{8} \mathrm{G}_{\mathbf{2}}$-Lösungen der Konzentrationen $50 \mathrm{mmol} / \mathrm{L}$ (blau), $75 \mathrm{mmol} / \mathrm{L}$ (rot) und $100 \mathrm{mmol} / \mathrm{L}$ (grün) in der $(\alpha \lambda)_{\text {exz }}$-Darstellung. Symbole wie in Abbildung 27. 
4 Ergebnisse und Auswertung

\begin{tabular}{|c|c|c|c|c|c|c|c|c|c|c|c|c|}
\hline $\begin{array}{c}c \\
{[\mathrm{mmol} / \mathrm{L}]}\end{array}$ & $X$ & $\begin{array}{c}\rho \\
{[\mathrm{g} / \mathrm{mL}]}\end{array}$ & $\begin{array}{c}c_{S} \\
{[\mathrm{~m} / \mathrm{s}]}\end{array}$ & $\begin{array}{l}\tau_{\text {mono }} \\
{[\mathrm{ns}]}\end{array}$ & $\begin{array}{l}A_{\text {mono }} \\
{\left[10^{-3}\right]}\end{array}$ & $\begin{array}{c}\tau_{\varphi \psi} \\
{[\mathrm{ns}]}\end{array}$ & $\begin{array}{c}A_{\varphi \psi} \\
{\left[10^{-3}\right]}\end{array}$ & $\begin{array}{c}\tilde{A}_{\varphi \psi} \\
{\left[10^{-7}\right.} \\
\cdot \mathrm{L} / \mathrm{mol}]\end{array}$ & $\begin{array}{c}\tau_{\omega} \\
{[\mathrm{ns}]}\end{array}$ & $\begin{array}{c}A_{\omega} \\
{\left[10^{-3}\right]}\end{array}$ & $\begin{array}{c}\tilde{A}_{\omega} \\
{\left[10^{-7}\right.} \\
\cdot \mathrm{L} / \mathrm{mol}]\end{array}$ & $\begin{array}{c}B \\
{[\mathrm{ps}]}\end{array}$ \\
\hline 50 & 1.2 & 1.002346 & 1502 & $963(27)$ & $5.75(14)$ & $6.5(13)$ & $0.12(3)$ & 0.42 & $0.7(3)$ & $0.7(4)$ & 2.44 & $34.7(3)$ \\
\hline 75 & 2.3 & 1.005038 & 1504 & $610(9)$ & $7.29(8)$ & $7.7(8)$ & $0.21(2)$ & 0.73 & $1.0(1)$ & $0.93(6)$ & 3.23 & $37.7(1)$ \\
\hline 100 & 3.4 & 1.007541 & 1505 & $462(6)$ & $8.18(8)$ & $9.2(9)$ & $0.25(2)$ & 0.86 & $1.1(1)$ & $1.20(6)$ & 4.15 & $40.9(2)$ \\
\hline
\end{tabular}

Tabelle 4.9: Parameter aus den Anpassungsrechnungen für die Ultraschallabsorptionsspektren von $\mathrm{C}_{8} \mathrm{G}_{2}$-Lösungen.

Auch bei diesem System weisen die Ultraschallabsorptionsspektren aller untersuchten Konzentrationen Beiträge aufgrund der $\omega$-Rotation, der $\varphi$-/ $\psi$-Rotation und des Monomeraustauschprozesses auf. 


\subsubsection{3 n-Nonyl- $\beta$-D-Maltopyranosid $\left(\mathrm{C}_{9} \mathrm{G}_{2}\right)$}

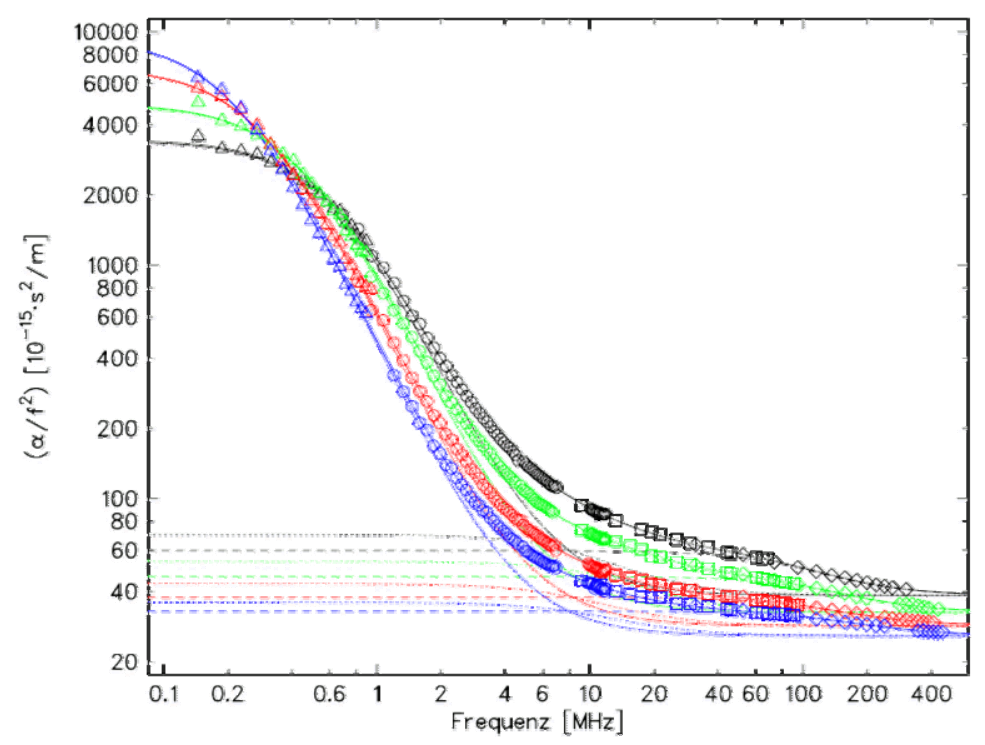

Abbildung 43: Ultraschallabsorptionsspektren von $\mathrm{C}_{9} \mathrm{G}_{2}$-Lösungen der Konzentrationen $75 \mathrm{mmol} / \mathrm{L}$ (blau), $100 \mathrm{mmol} / \mathrm{L}$ (rot), $150 \mathrm{mmol} / \mathrm{L}$ (grün) und $200 \mathrm{mmol} / \mathrm{L}$ (schwarz) in der ( $\alpha / f^{2}$ )-Darstellung. Die einzelnen Debye-Spektralterme sind wieder durch gestrichelte bzw. gepunktete Linien angedeutet. Symbole wie in Abbildung 27.

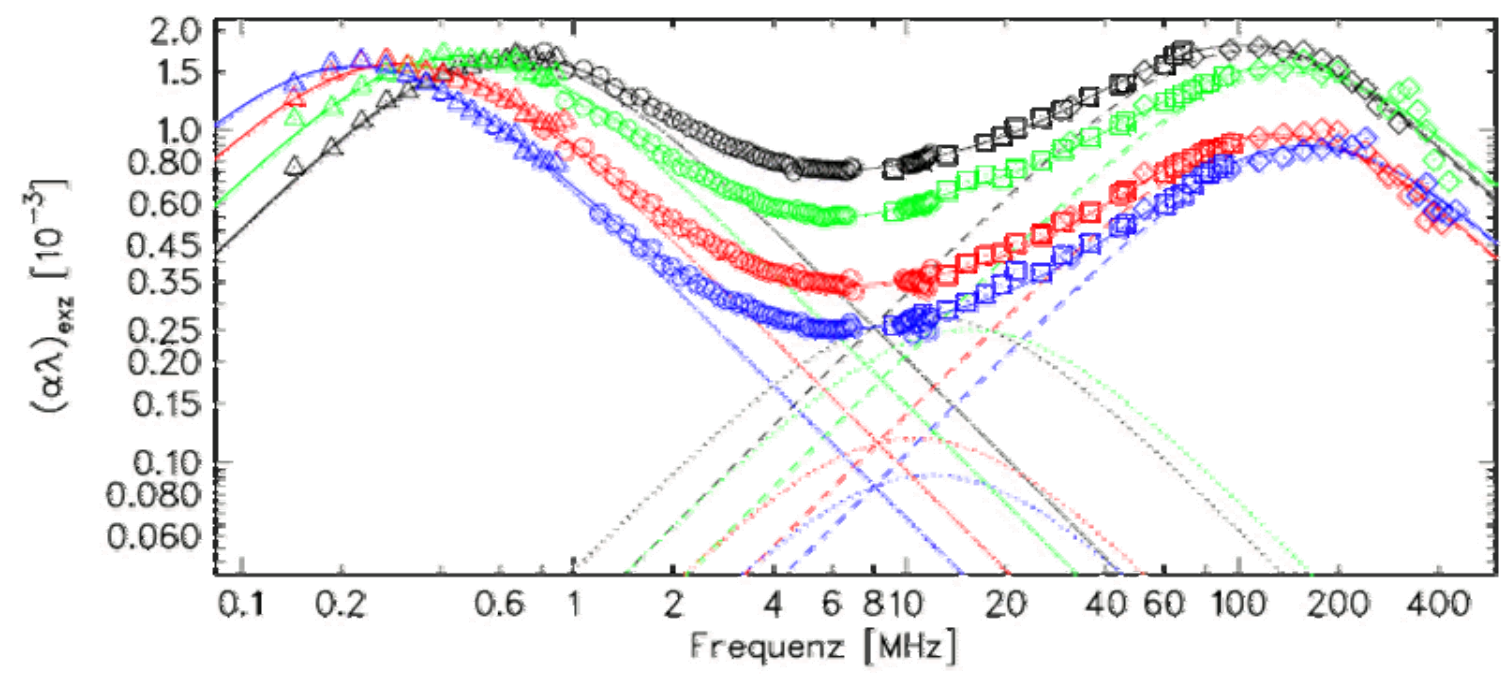

Abbildung 44: Ultraschallabsorptionsspektren von $\mathrm{C}_{9} \mathbf{G}_{2}$-Lösungen der Konzentrationen $75 \mathrm{mmol} / \mathrm{L}$ (blau), $100 \mathrm{mmol} / \mathrm{L}$ (rot), $150 \mathrm{mmol} / \mathrm{L}$ (grün) und $200 \mathrm{mmol} / \mathrm{L}$ (schwarz) in der $(\alpha \lambda)_{\mathrm{exz}}$-Darstellung. Symbole wie in Abbildung 27. 
4 Ergebnisse und Auswertung

\begin{tabular}{|c|c|c|c|c|c|c|c|c|c|c|c|c|}
\hline $\begin{array}{c}c \\
{[\mathrm{mmol} / \mathrm{L}]}\end{array}$ & $X$ & $\begin{array}{c}\rho \\
{[\mathrm{g} / \mathrm{mL}]}\end{array}$ & $\begin{array}{c}c_{S} \\
{[\mathrm{~m} / \mathrm{s}]}\end{array}$ & $\begin{array}{l}\tau_{\text {mono }} \\
{[\mathrm{ns}]}\end{array}$ & $\begin{array}{l}A_{\text {mono }} \\
{\left[10^{-3}\right]}\end{array}$ & $\begin{array}{c}\tau_{\varphi \psi} \\
{[\mathrm{ns}]}\end{array}$ & $\begin{array}{c}A_{\varphi \psi} \\
{\left[10^{-3}\right]}\end{array}$ & $\begin{array}{c}\tilde{A}_{\varphi \psi} \\
{\left[10^{-7}\right.} \\
\cdot \mathrm{L} / \mathrm{mol}]\end{array}$ & $\begin{array}{c}\tau_{\oplus} \\
{[\mathrm{ns}]}\end{array}$ & $\begin{array}{c}A_{\omega} \\
{\left[10^{-3}\right]}\end{array}$ & $\begin{array}{c}\tilde{A}_{\oplus} \\
{\left[10^{-7}\right.} \\
\cdot \mathrm{L} / \mathrm{mol}]\end{array}$ & $\begin{array}{c}B \\
{[\mathrm{ps}]}\end{array}$ \\
\hline 75 & 11.5 & 1.004255 & 1501 & $722(17)$ & $3.10(5)$ & $13.2(8)$ & $0.18(1)$ & 0.63 & $1.0(1)$ & $1.76(6)$ & 6.14 & $38.8(2)$ \\
\hline 100 & 15.7 & 1.006834 & 1502 & $530(1)$ & $3.15(4)$ & $15.0(8)$ & $0.24(1)$ & 0.83 & $1.2(1)$ & $1.89(5)$ & 6.57 & $42.8(1)$ \\
\hline 150 & 24 & 1.011729 & 1504 & $350(5)$ & $3.31(3)$ & $10.3(5)$ & $0.50(2)$ & 1.72 & 1.1(1) & $2.98(7)$ & 10.27 & $48.8(2)$ \\
\hline 200 & 32.3 & 1.016940 & 1506 & $249(3)$ & $3.22(2)$ & $13.8(6)$ & $0.55(2)$ & 1.88 & $1.5(1)$ & $3.46(7)$ & 11.84 & $57.9(2)$ \\
\hline
\end{tabular}

Tabelle 4.10: Parameter aus den Anpassungsrechnungen für die Ultraschallabsorptionsspektren von $\mathrm{C}_{9} \mathbf{G}_{2}$-Lösungen.

Die Spektren aller vier untersuchten Konzentrationen weisen jeweils drei Debye-Terme auf, die der $\omega$-Rotation, der $\varphi$-/ $\psi$-Rotation und dem Monomeraustauschprozess zugeordnet werden können. 


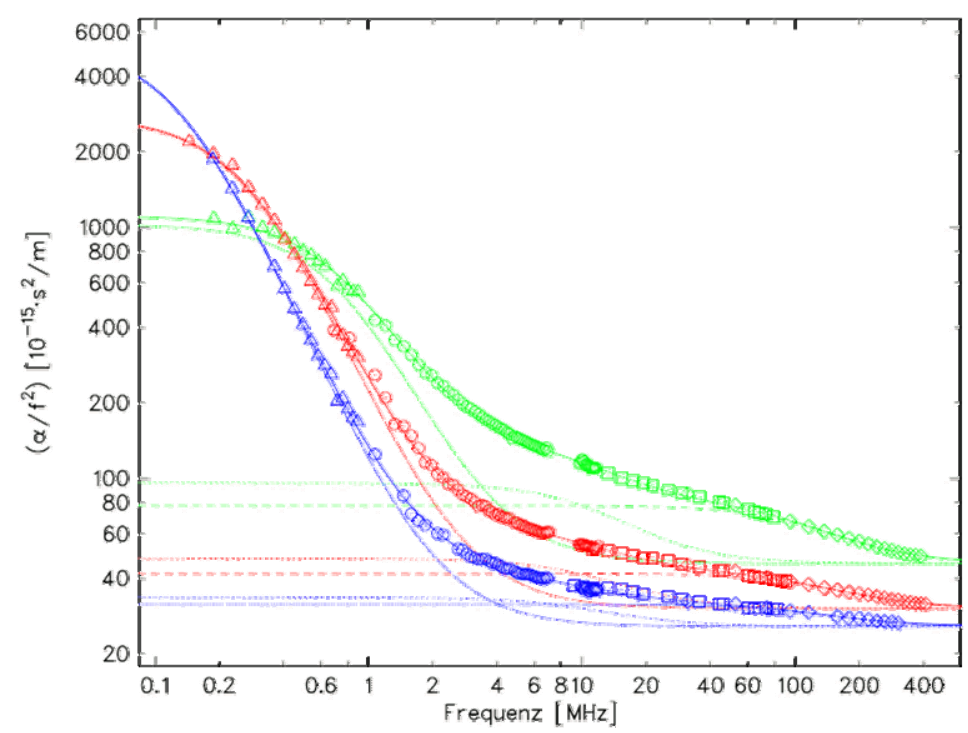

Abbildung 45: Ultraschallabsorptionsspektren von $C_{10} G_{2}$-Lösungen der Konzentrationen 50 $\mathrm{mmol} / \mathrm{L}$ (blau), $75 \mathrm{mmol} / \mathrm{L}$ (rot) und $100 \mathrm{mmol} / \mathrm{L}$ (grün) in der $\left(\boldsymbol{\alpha} / \boldsymbol{f}^{2}\right.$ )-Darstellung. Die einzelnen Debye-Spektralterme sind wieder durch gestrichelte bzw. gepunktete Linien angedeutet. Symbole wie in Abbildung 27.

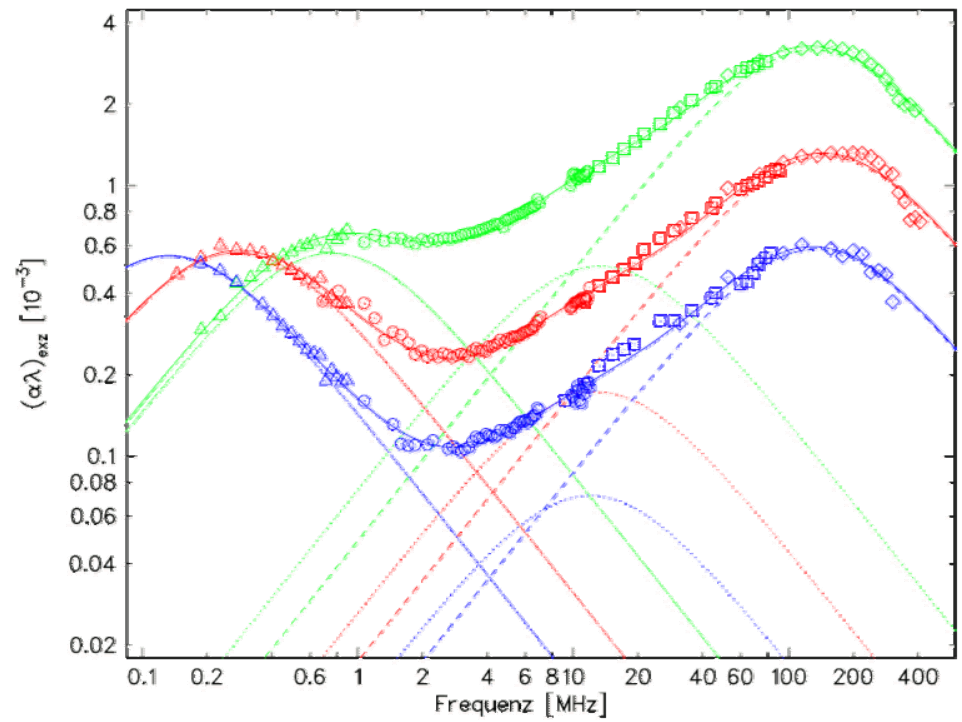

Abbildung 46: Ultraschallabsorptionsspektren von $C_{10} G_{2}$-Lösungen der Konzentrationen 50 $\mathrm{mmol} / \mathrm{L}$ (blau), $75 \mathrm{mmol} / \mathrm{L}$ (rot) und $100 \mathrm{mmol} / \mathrm{L}$ (grün) in der $(\alpha \lambda)_{\text {exz }}$-Darstellung. Symbole wie in Abbildung 27. 
4 Ergebnisse und Auswertung

\begin{tabular}{|c|c|c|c|c|c|c|c|c|c|c|c|c|}
\hline $\begin{array}{c}c \\
{[\mathrm{mmol} / \mathrm{L}]}\end{array}$ & $X$ & $\begin{array}{c}\rho \\
{[\mathrm{g} / \mathrm{mL}]}\end{array}$ & $\begin{array}{c}c_{S} \\
{[\mathrm{~m} / \mathrm{s}]}\end{array}$ & $\begin{array}{c}\tau_{\text {mono }} \\
{[\mathrm{ns}]}\end{array}$ & $\begin{array}{l}A_{\text {mono }} \\
{\left[10^{-3}\right]}\end{array}$ & $\begin{array}{c}\tau_{\varphi \psi} \\
{[\mathrm{ns}]}\end{array}$ & $\begin{array}{c}A_{\varphi \psi} \\
{\left[10^{-3}\right]}\end{array}$ & $\begin{array}{c}\tilde{A}_{\varphi \psi} \\
{\left[10^{-7}\right.} \\
\cdot \mathrm{L} / \mathrm{mol}]\end{array}$ & $\begin{array}{c}\tau_{\omega} \\
{[\mathrm{ns}]}\end{array}$ & $\begin{array}{c}A_{\omega} \\
{\left[10^{-3}\right]}\end{array}$ & $\begin{array}{c}\tilde{A}_{\omega} \\
{\left[10^{-7}\right.} \\
\cdot \mathrm{L} / \mathrm{mol}]\end{array}$ & $\begin{array}{c}B \\
{[\mathrm{ps}]}\end{array}$ \\
\hline 50 & 32 & 1.001922 & 1499 & $1202(81)$ & $1.10(6)$ & $13.2(1)$ & $0.14(1)$ & 0.49 & $1.2(1)$ & $1.16(9)$ & 4.07 & $38.5(3)$ \\
\hline 75 & 49 & 1.004383 & 1499 & $818(31)$ & $1.14(3)$ & 8.1(4) & $0.37(2)$ & 1.29 & $1.0(1)$ & $1.89(8)$ & 6.61 & $41.7(2)$ \\
\hline 100 & 66 & 1.006740 & 1501 & $574(16)$ & $1.13(2)$ & $12.2(6)$ & $0.35(1)$ & 1.22 & 1.1(1) & $2.57(9)$ & 8.94 & $45.5(2)$ \\
\hline 150 & 99 & 1.011361 & 1502 & $387(9)$ & $1.15(1)$ & $12.4(5)$ & $0.51(2)$ & 1.76 & $1.3(1)$ & $3.56(7)$ & 12.31 & $53.2(2)$ \\
\hline 200 & 132 & 1.016764 & 1504 & $286(6)$ & $1.11(1)$ & $14.1(5)$ & $0.67(2)$ & 2.30 & $1.4(1)$ & $4.85(7)$ & 16.64 & $61.6(2)$ \\
\hline 250 & 166 & 1.021544 & 1506 & $209(5)$ & $1.12(2)$ & $11.9(5)$ & $1.01(3)$ & 3.44 & $1.2(1)$ & $6.33(11)$ & 21.56 & $69.0(3)$ \\
\hline
\end{tabular}

Tabelle 4.11: Parameter aus den Anpassungsrechnungen für die Ultraschallabsorptionsspektren von $\mathrm{C}_{10} \mathrm{G}_{2}$-Lösungen.

Die Spektren aller sechs untersuchten Konzentrationen weisen wieder jeweils drei Debye-Terme auf, die der $\omega$ Rotation, der $\varphi-/ \psi$-Rotation und dem Monomeraustauschprozess zugeordnet werden können. 


\subsubsection{5 n-Decyl-a-D-Maltopyranosid $\left(a-C_{10} G_{2}\right)$}

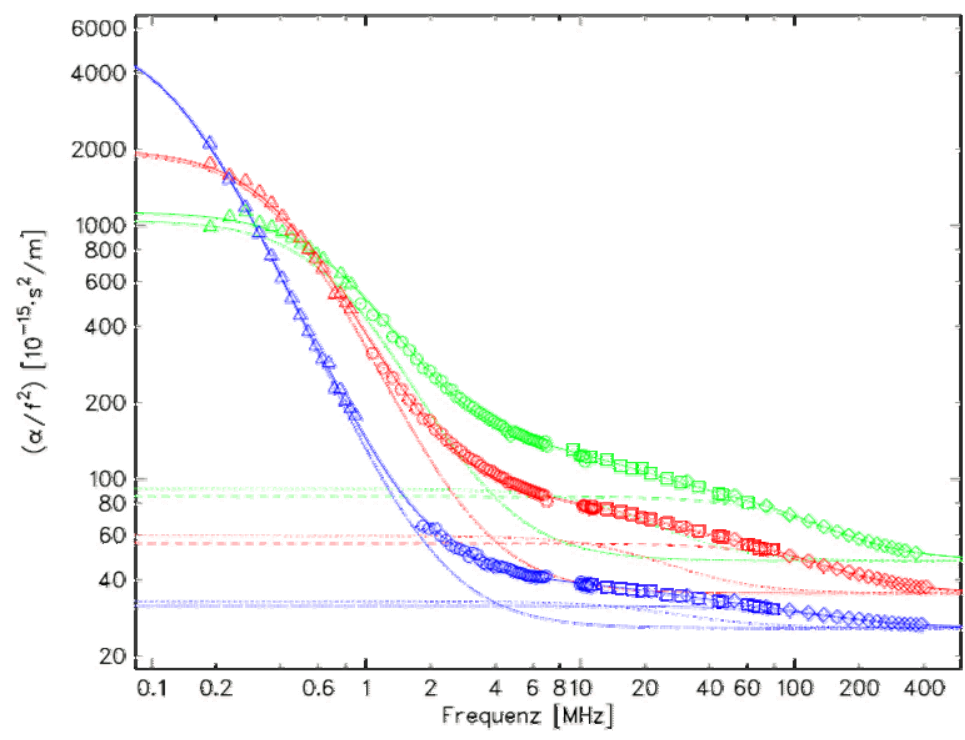

Abbildung 47: Ultraschallabsorptionsspektren von $\alpha-C_{10} G_{2}$-Lösungen der Konzentrationen 50 $\mathrm{mmol} / \mathrm{L}$ (blau), $150 \mathrm{mmol} / \mathrm{L}$ (rot) und $250 \mathrm{mmol} / \mathrm{L}$ (grün) in der ( $\alpha / \boldsymbol{f}^{\mathbf{2}}$ )-Darstellung. Die einzelnen Debye-Spektralterme sind wieder durch gestrichelte bzw. gepunktete Linien angedeutet. Symbole wie in Abbildung 27.

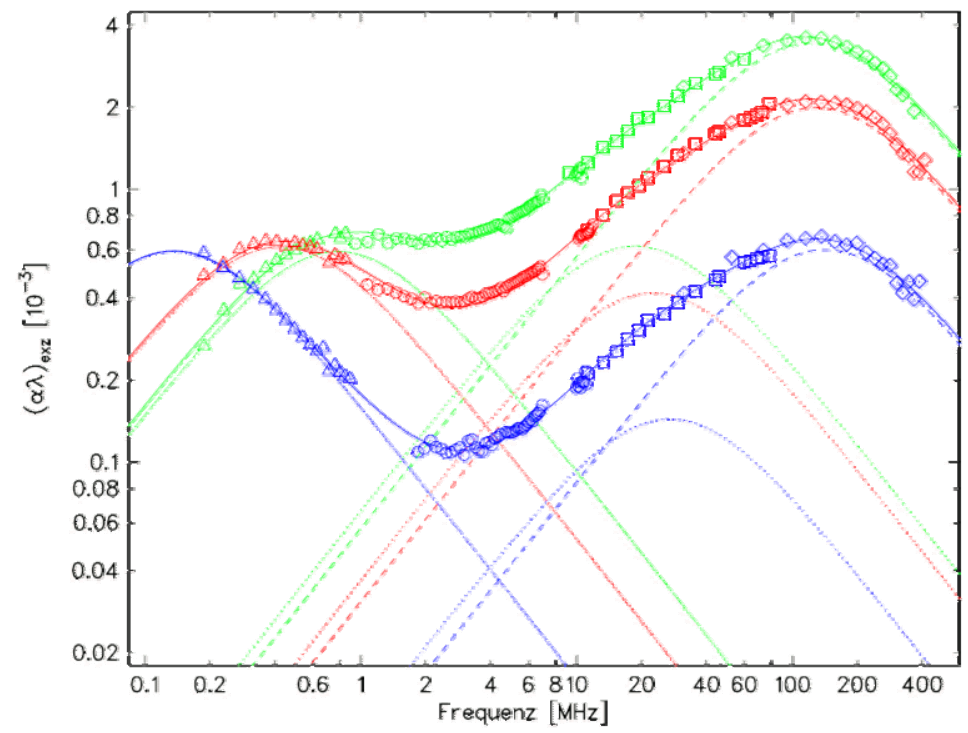

Abbildung 48: Ultraschallabsorptionsspektren von $\alpha-C_{10} G_{2}$-Lösungen der Konzentrationen 50 $\mathrm{mmol} / \mathrm{L}$ (blau), $150 \mathrm{mmol} / \mathrm{L}$ (rot) und $250 \mathrm{mmol} / \mathrm{L}$ (grün) in der $(\alpha \lambda)_{\mathrm{exz}}$-Darstellung. Symbole wie in Abbildung 27. 
4 Ergebnisse und Auswertung

\begin{tabular}{|c|c|c|c|c|c|c|c|c|c|c|c|c|}
\hline $\begin{array}{c}c \\
{[\mathrm{mmol} / \mathrm{L}]}\end{array}$ & $X$ & $\begin{array}{c}\rho \\
{[\mathrm{g} / \mathrm{mL}]}\end{array}$ & $\begin{array}{c}c_{S} \\
{[\mathrm{~m} / \mathrm{s}]}\end{array}$ & $\begin{array}{l}\tau_{\text {mono }} \\
{[\mathrm{ns}]}\end{array}$ & $\begin{array}{l}A_{\text {mono }} \\
{\left[10^{-3}\right]}\end{array}$ & $\begin{array}{c}\tau_{\varphi \psi} \\
{[\mathrm{ns}]}\end{array}$ & $\begin{array}{c}A_{\varphi \psi} \\
{\left[10^{-3}\right]}\end{array}$ & $\begin{array}{c}\tilde{A}_{\varphi \psi} \\
{\left[10^{-7}\right.} \\
\cdot \mathrm{L} / \mathrm{mol}]\end{array}$ & $\begin{array}{c}\tau_{\omega} \\
{[\mathrm{ns}]}\end{array}$ & $\begin{array}{c}A_{\omega} \\
{\left[10^{-3}\right]}\end{array}$ & $\begin{array}{c}\tilde{A}_{\omega} \\
{\left[10^{-7}\right.} \\
\cdot \mathrm{L} / \mathrm{mol}]\end{array}$ & $\begin{array}{c}B \\
{[\mathrm{ps}]}\end{array}$ \\
\hline 50 & 32 & 1.001963 & 1500 & 1173(63) & $1.18(5)$ & $5.9(5)$ & $0.29(3)$ & 1.02 & 1.1(1) & $1.20(5)$ & 4.20 & $38.7(2)$ \\
\hline 75 & 49 & 1.004361 & 1500 & $882(49)$ & $1.26(5)$ & $6.0(6)$ & $0.45(6)$ & 1.57 & $1.2(1)$ & $1.81(9)$ & 6.32 & $42.4(3)$ \\
\hline 100 & 65 & 1.007500 & 1502 & $567(14)$ & $1.23(2)$ & $6.0(3)$ & $0.67(5)$ & 2.33 & 1.1(1) & $2.54(7)$ & 8.82 & $46.2(2)$ \\
\hline 125 & 82 & 1.009491 & 1503 & 457(11) & $1.25(2)$ & $7.5(5)$ & $0.66(5)$ & 2.28 & $1.2(1)$ & $3.39(9)$ & 11.73 & $50.1(3)$ \\
\hline 150 & 99 & 1.011825 & 1502 & $375(9)$ & $1.23(2)$ & 7.1(4) & $0.83(6)$ & 2.87 & $1.2(1)$ & $3.99(8)$ & 13.79 & $53.1(2)$ \\
\hline 175 & 115 & 1.014235 & 1504 & $302(8)$ & $1.19(2)$ & 6.1(4) & $1.22(10)$ & 4.20 & 1.1(1) & $5.03(2)$ & 17.30 & $56.4(5)$ \\
\hline 200 & 132 & 1.016713 & 1505 & $261(6)$ & $1.19(1)$ & 7.3(4) & $1.14(7)$ & 3.91 & $1.3(1)$ & $5.24(11)$ & 17.95 & $61.6(4)$ \\
\hline 225 & 149 & 1.019310 & 1506 & $229(5)$ & $1.21(1)$ & $7.7(4)$ & $1.25(7)$ & 4.27 & $1.3(1)$ & $5.99(10)$ & 20.45 & $65.0(3)$ \\
\hline 250 & 165 & 1.021511 & 1507 & $203(5)$ & $1.18(2)$ & $8.5(5)$ & $1.24(8)$ & 4.22 & $1.3(1)$ & $6.04(13)$ & 20.54 & $71.6(4)$ \\
\hline
\end{tabular}

Tabelle 4.12: Parameter aus den Anpassungsrechnungen für die Ultraschallabsorptionsspektren von $\alpha-C_{10} G_{2}$-Lösungen.

Da in der Literatur keine Angaben über die $c m c$ von $\alpha-\mathrm{C}_{10} \mathrm{G}_{2}$ aufzufinden sind, wurden die reduzierten Konzentrationen für dieses System mit der $c m c$ von $\beta-\mathrm{C}_{10} \mathrm{G}_{2}(1.8 \mathrm{mmol} / \mathrm{L}([104]))$ berechnet. 


\subsection{3 n-Octyl- $\beta$-D-Galaktopyranosid ( $\left.\mathrm{C}_{8} \mathrm{Gal}\right)$}

Um den Einfluss der Stellung der OH-Gruppe am $\mathrm{C}_{5}$-Atom zu untersuchen, wurden Messungen an dem System n-Octyl- $\beta$-D-Galaktopyranosid vorgenommen. Da die Substanz sich allerdings bei gut messbaren Konzentrationen als nicht löslich erwies, wurden Mischungen mit dem Diastereomer $\mathrm{C}_{8} \mathrm{G}_{1}$ untersucht. Die Spektren der beiden untersuchten Mischungen und der reinen $\mathrm{C}_{8} \mathrm{G}_{1}$-Lösung sind in Abbildung 49 bzw. Abbildung 50 dargestellt.

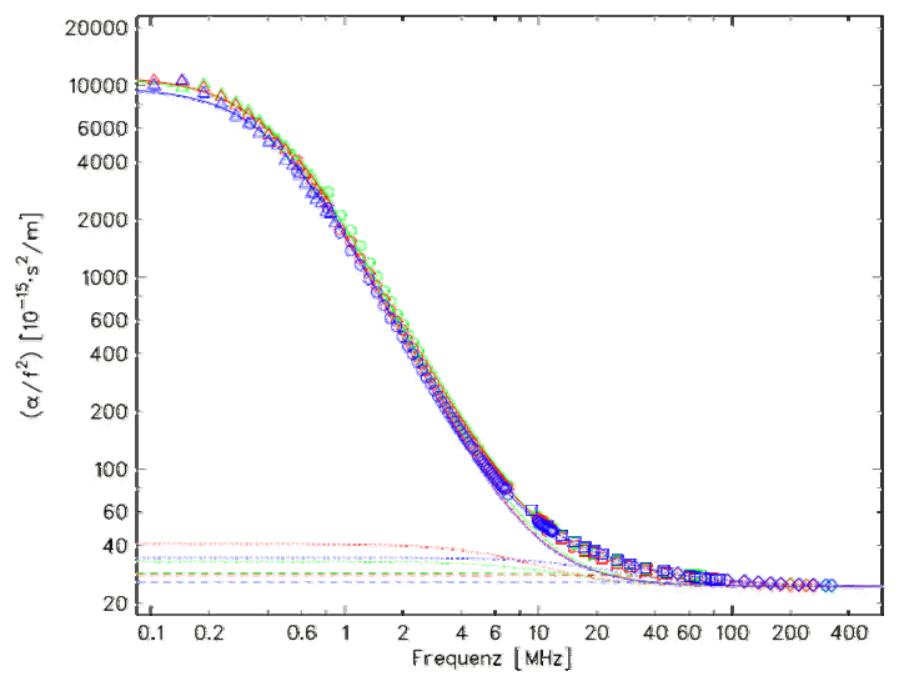

Abbildung 49: Ultraschallabsorptionsspektren für wässrige Lösungen von $100 \mathrm{mmol} / \mathrm{L}_{8} \mathrm{G}_{1}$ (blau), $50 \mathrm{mmol} / \mathrm{L} \mathrm{C}_{8} \mathrm{G}_{1}+50 \mathrm{mmol} / \mathrm{L} \mathrm{C}_{8} \mathrm{Gal}$ (rot) und $33 \mathrm{mmol} / \mathrm{L} \mathrm{C}_{8} \mathrm{G}_{1}+67 \mathrm{mmol} / \mathrm{L} \mathrm{C}_{8} \mathrm{Gal}$ (grün) in der $\left(\alpha / f^{2}\right)$-Darstellung. Die einzelnen Debye-Spektralterme sind wieder durch gestrichelte bzw. gepunktete Linien angedeutet. Symbole wie in Abbildung 27.

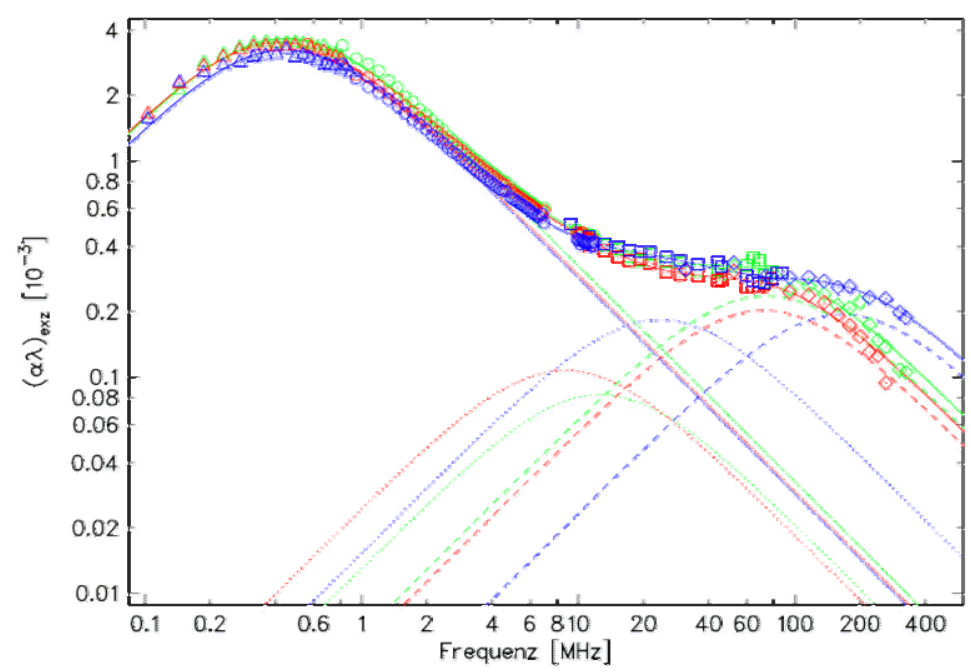

Abbildung 50: Ultraschallabsorptionsspektren für wässrige Lösungen von $100 \mathrm{mmol} / \mathrm{L}_{8} \mathrm{G}_{1}$ (blau), $50 \mathrm{mmol} / \mathrm{L} \mathrm{C}_{8} \mathrm{G}_{1}+50 \mathrm{mmol} / \mathrm{L} \mathrm{C}_{8} \mathrm{Gal}$ (rot) und $33 \mathrm{mmol} / \mathrm{L} \mathrm{C}_{8} \mathrm{G}_{1}+67 \mathrm{mmol} / \mathrm{L} \mathrm{C}_{8} \mathrm{Gal}$ (grün) in der $(\alpha \lambda)_{\text {exz }}$-Darstellung. Symbole wie in Abbildung 27. 
4 Ergebnisse und Auswertung

\begin{tabular}{r|r|c|c|c|c|c|c|c|c|c|c|c}
\hline $\begin{array}{c}c_{\mathrm{C} 8 \mathrm{Gal}} \\
{[\mathrm{mmol} / \mathrm{L}]}\end{array}$ & $X$ & $\begin{array}{c}\rho \\
{[\mathrm{g} / \mathrm{mL}]}\end{array}$ & $\begin{array}{c}c_{S} \\
{[\mathrm{~m} / \mathrm{s}]}\end{array}$ & $\begin{array}{c}\tau_{\text {mono }} \\
{[\mathrm{ns}]}\end{array}$ & $\begin{array}{c}A_{\text {mono }} \\
{\left[10^{-3}\right]}\end{array}$ & $\begin{array}{c}\tau_{\varphi \psi} \\
{[\mathrm{ns}]}\end{array}$ & $\begin{array}{c}A_{\varphi \psi} \\
{\left[10^{-3}\right]}\end{array}$ & $\begin{array}{c}\tilde{A}_{\varphi \psi} \\
{\left[10^{-7} .\right.}\end{array}$ & $\begin{array}{c}\tau_{\omega} \\
\mathrm{L} / \mathrm{mol}]\end{array}$ & $\begin{array}{c}A_{\omega} \\
{[\mathrm{ns}]}\end{array}$ & $\begin{array}{c}\tilde{A}_{\omega} \\
{\left[10^{-3}\right]}\end{array}$ & $\begin{array}{c}B \\
\mathrm{~L} / \mathrm{mol}]\end{array}$ \\
{$[\mathrm{ps}]$}
\end{tabular}

Tabelle 4.13: Parameter aus den Anpassungsrechnungen für die Ultraschallabsorptionsspektren von $\mathbf{C}_{8} \mathbf{G}_{1} / \mathbf{C}_{8} \mathbf{G a l}$-Lösungen. Die Gesamt-Tensidkonzentration beträgt für alle drei Mischungen 100 $\mathrm{mmol} / \mathrm{L}$.

$\mathrm{Da}$ auch für diese Mischungen in der Literatur keine $\mathrm{cmc}$-Werte vorliegen, wurde für die Berechnung der reduzierten Konzentration angenommen, dass die $c m c$ der Lösungen der Tensid-Mischungen linear zwischen den $c m c$-Werten der Lösungen der jeweiligen einzelnen Tenside $\left(c m c_{\mathrm{C} 8 \mathrm{G} 1} \approx 25 \mathrm{mmol} / \mathrm{L}, c m c_{\mathrm{C} 8 \mathrm{Gal}} \approx 29.8 \mathrm{mmol} / \mathrm{L}\right)$ verläuft:

$$
c m c_{\text {Mischung }}=\frac{c_{\mathrm{C} 8 \mathrm{G} 1}}{100 \mathrm{mmol} / \mathrm{L}} \cdot c m c_{\mathrm{C} 8 \mathrm{G} 1}+\frac{c_{\mathrm{C} 8 \mathrm{Gal}}}{100 \mathrm{mmol} / \mathrm{L}} \cdot c m c_{\mathrm{C} 8 \mathrm{Gal}}
$$




\subsection{4 a-Cyclodextrin/n-Octyl- $\beta$-D-Glukopyranosid-Mischungen (a- $\left.\mathrm{CD}+\mathrm{C}_{8} \mathrm{G}_{1}\right)$}

Die Spektren der $\mathrm{C}_{8} \mathrm{G}_{1} / \alpha$-CD-Lösungen lassen sich, wie auch die reinen $\mathrm{C}_{8} \mathrm{G}_{1}$-Spektren, zufriedenstellend mit einer Summe aus drei Debye-Spektraltermen anpassen. Drei der entsprechenden Spektren sind in Abbildung 51 bzw. Abbildung 52 dargestellt und die resultierenden Parameter der Anpassungen sind inTabelle 4.14 aufgelistet.

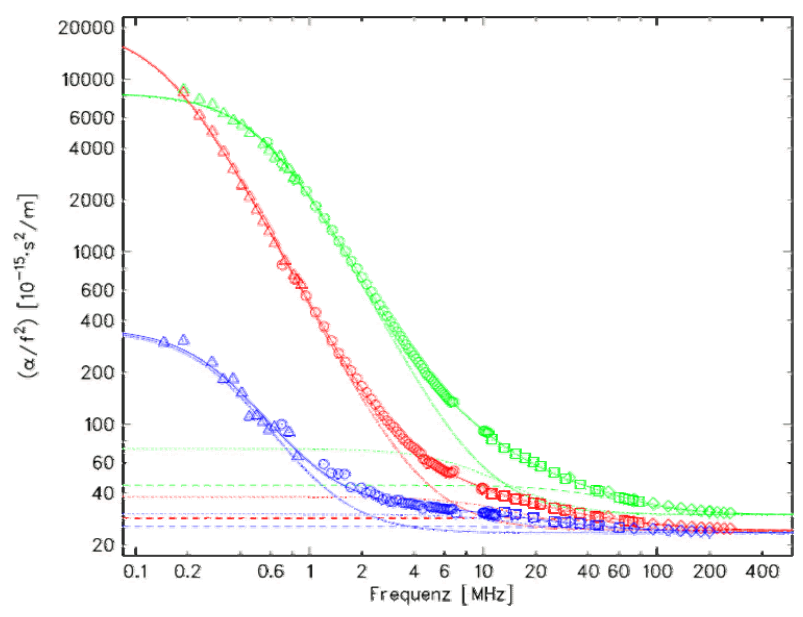

Abbildung 51: Ultraschallabsorptionsspektren für wässrige Lösungen von 16 mmol/L $\mathrm{C}_{8} \mathrm{G}_{1}+50$ $\mathrm{mmol} / \mathrm{L} \mathrm{\alpha -CD} \mathrm{(blau),} 87.5 \mathrm{mmol} / \mathrm{L} \mathrm{C}_{8} \mathrm{G}_{1}+50 \mathrm{mmol} / \mathrm{L} \alpha$-CD (rot) und $200 \mathrm{mmol} / \mathrm{L} \mathrm{C}_{8} \mathrm{G}_{1}+50$ $\mathbf{m m o l} / \mathrm{L} \boldsymbol{\alpha}-\mathrm{CD}$ (grün) in der $\left(\boldsymbol{\alpha} / \boldsymbol{f}^{2}\right)$-Darstellung. Die einzelnen Debye-Spektralterme sind wieder durch gestrichelte bzw. gepunktete Linien angedeutet. Symbole wie in Abbildung 27. Anpassung mit drei Debye-Spektraltermen.

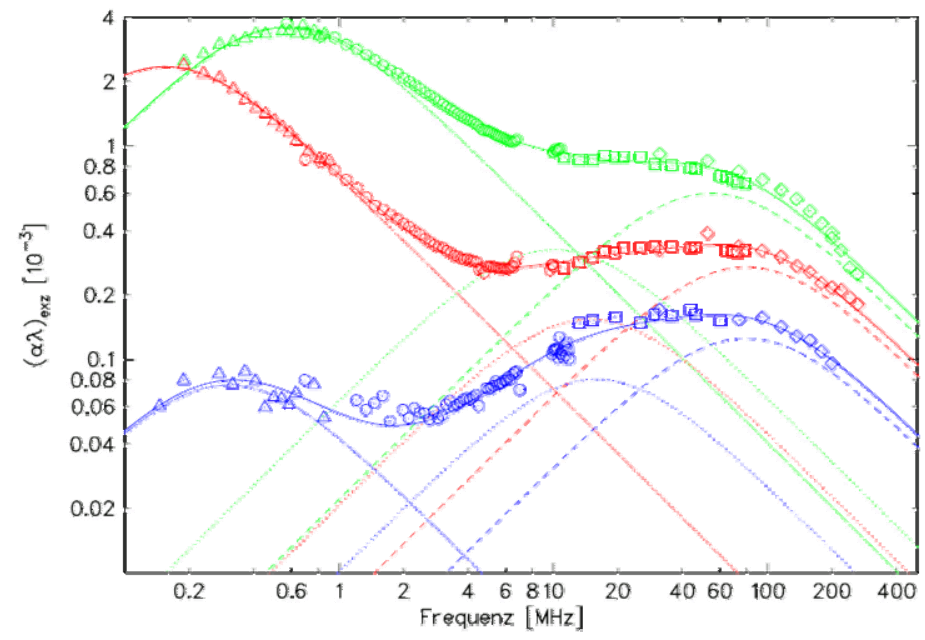

Abbildung 52: Ultraschallabsorptionsspektren für wässrige Lösungen von $16 \mathrm{mmol} / \mathrm{L} \mathrm{C}_{8} \mathrm{G}_{1}+50$ $\mathrm{mmol} / \mathrm{L} \mathrm{\alpha -CD} \mathrm{(blau),} 87.5 \mathrm{mmol} / \mathrm{L} \mathrm{C}_{8} \mathrm{G}_{1}+50 \mathrm{mmol} / \mathrm{L} \alpha$-CD (rot) und $200 \mathrm{mmol} / \mathrm{L} \mathrm{C}_{8} \mathrm{G}_{1}+50$ $\mathbf{m m o l} / \mathbf{L} \alpha-C D$ (grün) in der $(\alpha \lambda)_{\text {exz }}$-Darstellung. Symbole wie in Abbildung 27. Anpassung mit drei Debye-Spektraltermen. 
4 Ergebnisse und Auswertung

\begin{tabular}{|c|c|c|c|c|c|c|c|c|c|c|c|c|}
\hline $\begin{array}{c}c_{\mathrm{C} 8 \mathrm{G} 1} \\
{[\mathrm{mmol} / \mathrm{L}]}\end{array}$ & $\begin{array}{c}c_{\alpha-\mathrm{CD}} \\
{[\mathrm{mmol} / \mathrm{L}]}\end{array}$ & $\begin{array}{c}\rho \\
{[\mathrm{g} / \mathrm{mL}]}\end{array}$ & $\begin{array}{c}c_{S} \\
{[\mathrm{~m} / \mathrm{s}]}\end{array}$ & $\begin{array}{l}\tau_{\text {mono }} \\
{[\mathrm{ns}]}\end{array}$ & $\begin{array}{l}A_{\text {mono }} \\
{\left[10^{-3}\right]}\end{array}$ & $\begin{array}{c}\tau_{\varphi \psi} \\
{[\mathrm{ns}]}\end{array}$ & $\begin{array}{c}A_{\oplus \Psi} \\
{\left[10^{-3}\right]}\end{array}$ & $\begin{array}{c}\tilde{A}_{\varphi \psi} \\
{\left[10^{-7}\right.} \\
\cdot \mathrm{L} / \mathrm{mol}]\end{array}$ & $\begin{array}{c}\tau_{\omega} \\
{[\mathrm{ns}]}\end{array}$ & $\begin{array}{c}A_{\omega} \\
{\left[10^{-3}\right]}\end{array}$ & $\begin{array}{c}\tilde{A}_{\omega} \\
{\left[10^{-7}\right.} \\
\cdot \mathrm{L} / \mathrm{mol}]\end{array}$ & $\begin{array}{c}B \\
{[\mathrm{ps}]}\end{array}$ \\
\hline 16 & 50 & 1.01544 & 1508 & $610(5)$ & $0.14(1)$ & $63(2)$ & $0.04(1)$ & 0.1 & $4.6(3)$ & $0.35(1)$ & 1.2 & $35.6(1)$ \\
\hline 33 & 50 & 1.015982 & 1510 & $709(48)$ & $0.26(1)$ & $9.2(7)$ & $0.21(2)$ & 0.7 & $2 *$ & $0.32(3)$ & 1.1 & $35.4(1)$ \\
\hline 57.5 & 50 & 1.017294 & 1514 & $839(35)$ & $0.30(9)$ & $9.0(7)$ & $0.24(2)$ & 0.8 & $2 *$ & $0.39(3)$ & 1.3 & $35.6(1)$ \\
\hline 65 & 50 & 1.017528 & 1516 & 951(91) & $0.34(2)$ & $8.4(23)$ & $0.25(15)$ & 0.8 & $2.8(11)$ & $0.39(13)$ & 1.3 & $35.9(2)$ \\
\hline 75 & 50 & 1.017755 & 1516 & $1021(47)$ & $1.67(6)$ & $8.4(6)$ & $0.31(20)$ & 1.1 & $2^{*}$ & $0.40(3)$ & 1.1 & $36.2(1)$ \\
\hline 87.5 & 50 & 1.017993 & 1517 & $974(56)$ & $4.48(22)$ & $10.5(8)$ & $0.31(2)$ & 1.0 & $2 *$ & $0.53(3)$ & 1.8 & $36.9(1)$ \\
\hline 100 & 50 & 1.018568 & 1516 & $869(35)$ & $5.82(19)$ & $10.2(12)$ & $0.35(6)$ & 1.3 & $2.5(4)$ & $0.52(4)$ & 1.8 & $37.6(1)$ \\
\hline 112.5 & 50 & 1.019066 & 1517 & $622(14)$ & $6.45(11)$ & $12.6(10)$ & $0.32(3)$ & 1.1 & $2.6(2)$ & $0.65(2)$ & 2.2 & $38.9(1)$ \\
\hline 125 & 50 & 1.019868 & 1517 & $480(17)$ & $6.55(17)$ & 7.4(7) & $0.66(6)$ & 2.2 & $1.2(3)$ & $0.71(1)$ & 2.4 & $38.9(3)$ \\
\hline 137.5 & 50 & 1.021001 & 1518 & 454(19) & $6.90(19)$ & $14.5(24)$ & $0.40(6)$ & 1.3 & $2.8(4)$ & $0.80(5)$ & 2.7 & $40.8(2)$ \\
\hline 200 & 50 & 1.022159 & 1518 & $280(8)$ & 7.13(11) & $15.7(21)$ & $0.65(6)$ & 2.2 & $2.9(3)$ & $1.20(6)$ & 4.0 & $45.4(2)$ \\
\hline
\end{tabular}

Tabelle 4.14: Parameter aus den Anpassungsrechnungen für die Ultraschallabsorptionsspektren von $\mathrm{C}_{8} \mathrm{G}_{1} / \alpha$-CD-Lösungen bei Anpassung mit drei Debye-Spektraltermen.

Eine genauere Betrachtung der Spektren ergibt allerdings, dass bei der Anpassung mit drei DebyeSpektralfunktionen bei allen Lösungen systematische Abweichungen der Anpassungen von den Messpunkten auftreten. Durch Zunahme eines vierten Debye-Spektralterms bei der Anpassung lässt sich diese Systematik deutlich verringern, wie aus Abbildung 53 exemplarisch ersichtlich wird.
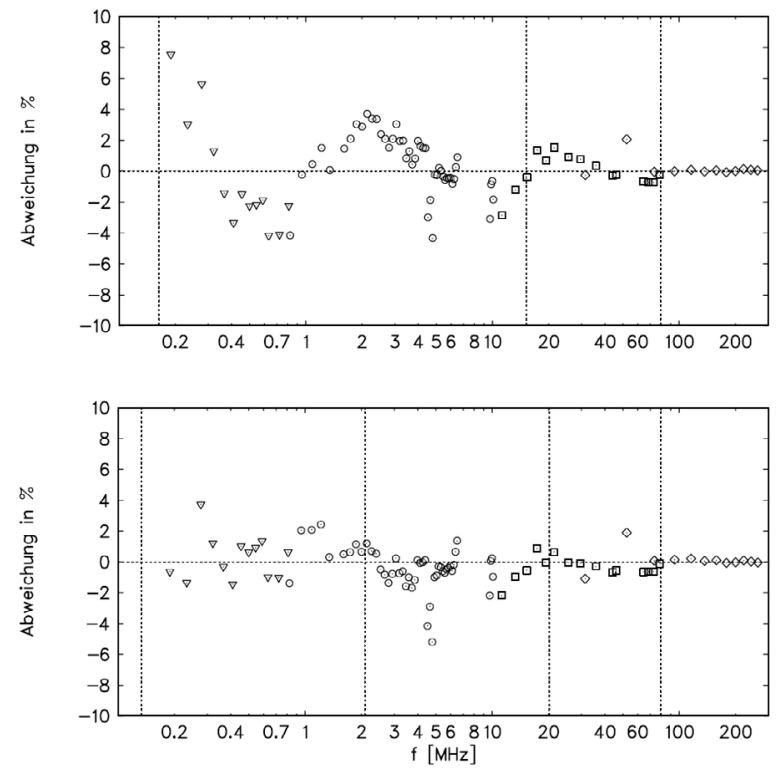

Abbildung 53: Abweichungen der Messwerte von den Spektralfunktionen für eine Beschreibung der Spektren mit drei (oben) bzw. vier (unten) Debye-Relaxationstermen für die Lösung 87.5 $\mathrm{mmol} / \mathrm{L} \mathrm{C}_{8} \mathrm{G}_{1}+50 \mathrm{mmol} / \mathrm{L} \alpha-\mathrm{CD}$. 
Daher wird alternativ eine Anpassung mit einer Summe aus vier Debye-Spektraltermen vorgenommen. Da die daraus resultierenden Funktionen durchgehend weniger systematisch von den Messpunkten abweichen als jene mit drei Debye-Spektraltermen und da sich zudem die resultierenden Parameter als sinnvoll und untereinander konsistent erweisen (siehe Kapitel 5.6), wird bei der Diskussion ausschließlich diese Beschreibung der Spektren behandelt. In Abbildung 54 bzw. Abbildung 55 sind daher noch einmal drei Ultraschallabsorptionsspektren von $\mathrm{C}_{8} \mathrm{G}_{1} / \alpha$-CD-Lösungen dargestellt, die mit vier Debye-Spektralfunktionen beschrieben werden, und in Tabelle 4.15 sind die entsprechenden Parameter aufgelistet, wobei der zusätzliche vierte Term mit dem Index ,kompl' versehen ist, da er in Kapitel 5.6.2 der Komplexbildung zugeschrieben wird. Allerdings muss hier daraufhingewiesen werden, dass auch die Anpassung mit drei Debye-Spektraltermen die Daten im Rahmen der Messfehler adäquat beschreibt.

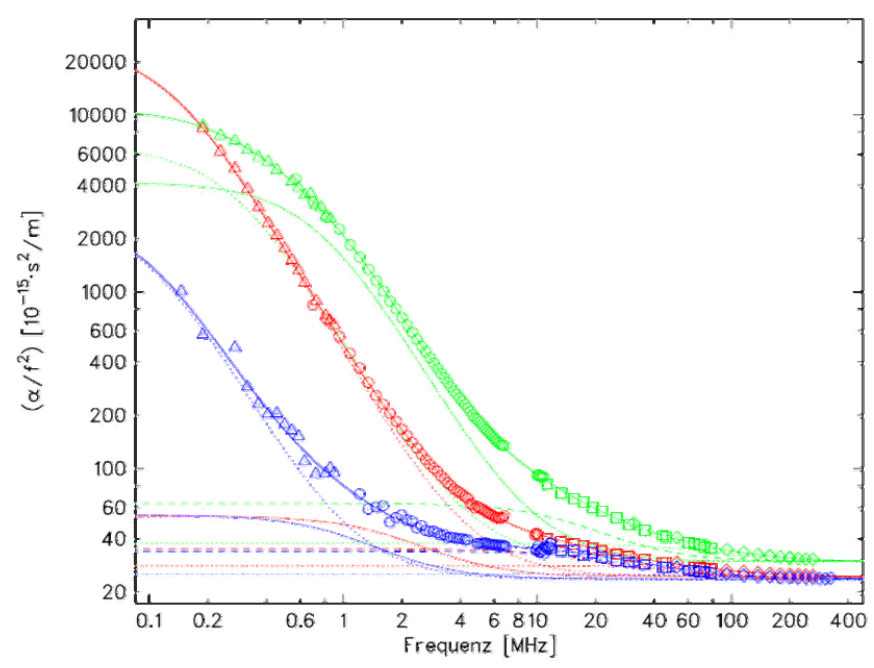

Abbildung 54: Ultraschallabsorptionsspektren für Lösungen von $16 \mathrm{mmol} / \mathrm{L} \mathrm{C}_{8} \mathrm{G}_{1}+65 \mathrm{mmol} / \mathrm{L} \alpha-$ CD (blau), $87.5 \mathrm{mmol} / \mathrm{L} \mathrm{C}_{8} \mathrm{G}_{1}+50 \mathrm{mmol} / \mathrm{L} \mathrm{\alpha -CD}$ (rot) und $200 \mathrm{mmol} / \mathrm{L} \mathrm{C}_{8} \mathrm{G}_{1}+50 \mathrm{mmol} / \mathrm{L} \mathrm{\alpha -CD}$ (grün) in der $\left(\boldsymbol{\alpha} / \boldsymbol{f}^{2}\right)$-Darstellung. Symbole wie in Abbildung 27. Anpassung mit vier DebyeSpektraltermen.

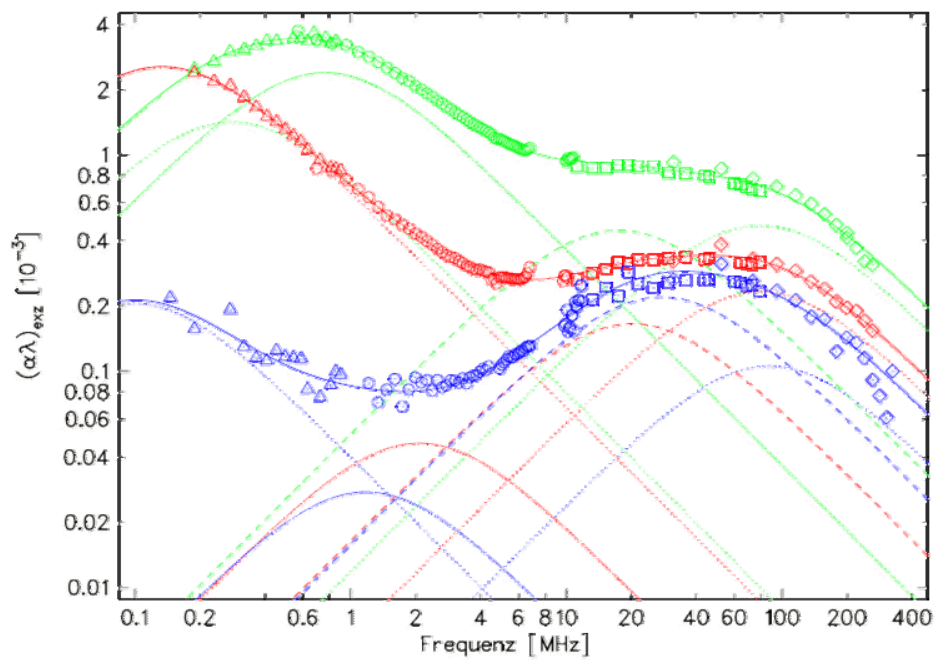

Abbildung 55: Ultraschallabsorptionsspektren für Lösungen von $16 \mathrm{mmol} / \mathrm{L} \mathrm{C}_{8} \mathrm{G}_{1}+65 \mathrm{mmol} / \mathrm{L} \alpha-\mathrm{CD}$ (blau), $87.5 \mathrm{mmol} / \mathrm{L} \mathrm{C}_{8} \mathrm{G}_{1}+50 \mathrm{mmol} / \mathrm{L} \alpha-\mathrm{CD}$ (rot) und $200 \mathrm{mmol} / \mathrm{L} \mathrm{C}_{8} \mathrm{G}_{1}+50 \mathrm{mmol} / \mathrm{L} \alpha-\mathrm{CD}$ (grün) in $\operatorname{der}(\alpha \lambda)_{\text {exz }}$-Darstellung. Symbole wie in Abbildung 27. Anpassung mit vier Debye-Spektraltermen. 
4 Ergebnisse und Auswertung

\begin{tabular}{|c|c|c|c|c|c|c|c|c|c|c|c|c|}
\hline $\begin{array}{c}c_{\mathrm{C} 8 \mathrm{Gl}} \\
{[\mathrm{mmol} /} \\
\mathrm{L}]\end{array}$ & $\begin{array}{l}\tau_{\text {mono }} \\
{[\mathrm{ns}]}\end{array}$ & $\begin{array}{l}A_{\text {mono }} \\
{\left[10^{-3}\right]}\end{array}$ & $\begin{array}{l}\tau_{\text {kompl }} \\
{[\mathrm{ns}]}\end{array}$ & $\begin{array}{c}A_{k o m p l} \\
{\left[10^{-3}\right]}\end{array}$ & $\begin{array}{c}\tilde{A}_{\text {kompl }} \\
{\left[10^{-7}\right.} \\
\cdot \mathrm{L} / \mathrm{mol}]\end{array}$ & $\begin{array}{c}\tau_{\varphi \psi} \\
{[\mathrm{ns}]}\end{array}$ & $\begin{array}{c}A_{\varphi \psi} \\
{\left[10^{-3}\right]}\end{array}$ & $\begin{array}{c}\tilde{A}_{\varphi \psi} \\
{\left[10^{-7}\right.} \\
\cdot \mathrm{L} / \mathrm{mol}]\end{array}$ & $\begin{array}{c}\tau_{\omega} \\
{[\mathrm{ns}]}\end{array}$ & $\begin{array}{c}A_{\omega} \\
{\left[10^{-3}\right]}\end{array}$ & $\begin{array}{c}\tilde{A}_{\omega} \\
{\left[10^{-7}\right.} \\
\cdot \mathrm{L} / \mathrm{mol}]\end{array}$ & $B \quad[\mathrm{ps}]$ \\
\hline 16 & $663(69)$ & $0.14(1)$ & $104(36)$ & $0.04(9)$ & 0.1 & $7.0(12)$ & $0.20(4)$ & 0.7 & $2 *$ & $0.17(7)$ & 0.6 & $35.6(2)$ \\
\hline 33 & $650(68)$ & $0.21(1)$ & $33(2)$ & $0.03(2)$ & 0.1 & $4.8(14)$ & $0.35(13)$ & 1.2 & $2 *$ & $0.13(17)$ & 0.4 & $35.5(2)$ \\
\hline 57.5 & $870(229)$ & $0.35(5)$ & $94(29)$ & $0.05(1)$ & 0.2 & $6.1(9)$ & $0.32(9)$ & 1.1 & $1.9(7)$ & $0.27(6)$ & 0.9 & $35.7(1)$ \\
\hline 65 & $1625(472)$ & $0.41(7)$ & $135(49)$ & $0.06(1)$ & 0.2 & $5.7(12)$ & $0.44(17)$ & 1.5 & $1.8(16)$ & $0.21(12)$ & 0.7 & $35.9(2)$ \\
\hline 75 & 1289(99) & $1.80(8)$ & $114(28)$ & $0.09(2)$ & 0.3 & $6.0(7)$ & $0.42(7)$ & 1.4 & $1.6(5)$ & $0.29(5)$ & 1 & $36.2(1)$ \\
\hline 87.5 & 1192(95) & $5.10(30)$ & $76(3)$ & $0.09(3)$ & 0.3 & 7.9(9) & $0.33(2)$ & 1.1 & $2 *$ & $0.47(4)$ & 1.6 & $37.0(1)$ \\
\hline 100 & $941(6)$ & $5.95(21)$ & $91(59)$ & $0.10(6)$ & 0.3 & $7.4(11)$ & $0.47(8)$ & 1.6 & $1.8(5)$ & $0.44(6)$ & 1.5 & $37.4(2)$ \\
\hline 112.5 & $651(18)$ & $6.45(1)$ & $59(19)$ & $0.12(3)$ & 0.4 & $7.7(6)$ & $0.43(2)$ & 1.4 & $2 *$ & $0.52(4)$ & 1.7 & $38.8(1)$ \\
\hline 125 & $519(31)$ & $6.60(18)$ & $61(49)$ & $0.15(9)$ & 0.5 & $5.9(7)$ & $0.75(7)$ & 2.5 & $0.8(3)$ & $0.76(23)$ & 2.6 & $38.6(5)$ \\
\hline 137.5 & $470(38)$ & $6.9(3)$ & $81(114)$ & $0.16(9)$ & 0.5 & $9.8(18)$ & $0.50(4)$ & 1.7 & $2 *$ & $0.73(8)$ & 2.4 & $40.5(2)$ \\
\hline 200 & $277(6)$ & $7.03(10)$ & $42(32)$ & $0.2 *$ & 0.7 & $8.5(11)$ & $0.87(6)$ & 3 & $2 *$ & $0.92(7)$ & 3.2 & $45.2(1)$ \\
\hline
\end{tabular}

Tabelle 4.15: Parameter aus den Anpassungsrechnungen für die Ultraschallabsorptionsspektren von $\mathrm{C}_{8} \mathrm{G}_{1} / \alpha$-CD-Lösungen bei Anpassung mit vier Debye-Spektraltermen. 


\section{Diskussion}

\subsection{Der Monomeraustauschprozess}

Die tieffrequenten Absorptionsanteile in den Ultraschallabsorptionsspektren der Alkylglykosid-Lösungen können durchgehend dem Monomeraustauschprozess zugeordnet werden. Als Beleg dafür sind in Abbildung 56 die Spektren von Glukose, Maltose und $\mathrm{C}_{6} \mathrm{G}_{1}$ (jeweils $200 \mathrm{mmol} / \mathrm{L}$ ) aufgetragen, von denen nur letzteres einen tieffrequenten Absorptionsanteil aufweist. Während Glukose und Maltose generell keine Mizellen bilden, liegt ein Teil der $\mathrm{C}_{6} \mathrm{G}_{1}$-Moleküle bei dieser Konzentration in Mizellen vor, so dass entsprechend auch nur für die $\mathrm{C}_{6} \mathrm{G}_{1}$-Lösung ein Monomeraustauschprozess zu erwarten ist.

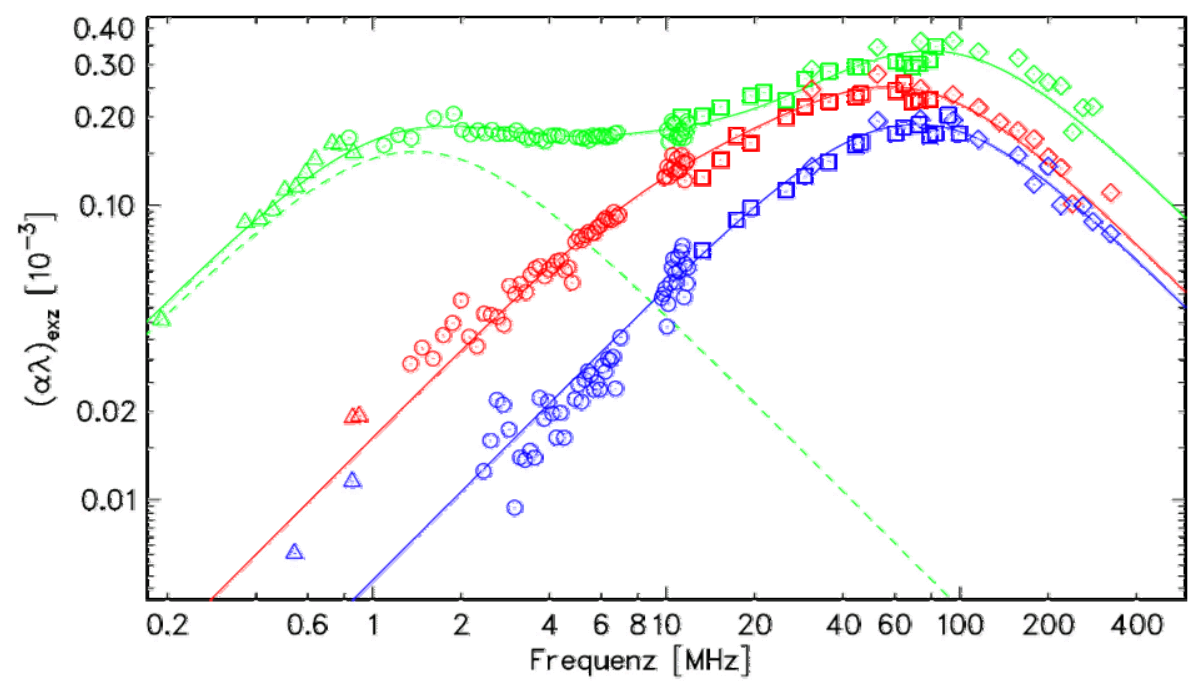

Abbildung 56: Ultraschallabsorptionsspektren für Lösungen von jeweils $200 \mathrm{mmol} / \mathrm{L}$ Glukose (blau), Maltose (rot) und $\mathbf{C}_{\mathbf{6}} \mathbf{G}_{\mathbf{1}}$ (grün). Die gestrichelte Linie beschreibt die dem Monomeraustauschprozess der $\mathrm{C}_{6} \mathrm{G}_{1}$-Lösungen zuzuordnende Debye-Spektralfunktion.

Einen weiteren Beleg für die Zuordnung des tieffrequenten Relaxationsterms zum Monomeraustauschprozess liefern die Spektren der Systeme, bei denen sowohl unterhalb als auch oberhalb der $c m c$ gemessen wurde. Wie zu erwarten ist, weisen nur jene Spektren einen tieffrequenten Absorptionsanteil auf, bei denen die Konzentration oberhalb bzw. dicht unterhalb der jeweiligen $c m c$ liegt (siehe Abbildung 31 bis Abbildung 34 und Abbildung 39 bzw. Abbildung 40).

Unerwartet hingegen ist zunächst die Notwendigkeit von zwei Debye-Spektraltermen zur adäquaten Beschreibung des tieffrequenten Teils einiger Spektren. Interessant ist hier vor allem die Tatsache, dass dieser zweite Absorptionsbeitrag bei zwei Tensiden im gesamten betrachteten Konzentrationsbereich oberhalb der $\mathrm{cmc}$ auftritt $\left(\mathrm{C}_{7} \mathrm{G}_{1}: 100 \mathrm{mmol} / \mathrm{L}, 150 \mathrm{mmol} / \mathrm{L}, 200 \mathrm{mmol} / \mathrm{L}\right.$; siehe Tabelle 4.4 und $\mathrm{C}_{9} \mathrm{G}_{1}: 75 \mathrm{mmol} / \mathrm{L}, 100 \mathrm{mmol} / \mathrm{L}, 150$ mmol/L, 200 mmol/L; siehe Tabelle 4.7), bei zwei weiteren Substanzen jeweils nur bei einer der untersuchten Konzentrationen $\left(\mathrm{C}_{8} \mathrm{G}_{1}: 200 \mathrm{mmol} / \mathrm{L}\right.$; siehe Tabelle 4.5; $\mathrm{C}_{6} \mathrm{G}_{2}: 200 \mathrm{mmol} / \mathrm{L}$, siehe Tabelle 4.8) und bei den restlichen Tensiden überhaupt nicht.

Das Auftreten eines zweiten Monomeraustauschprozesses bei dem System $\mathrm{C}_{6} \mathrm{G}_{2}$ wird einer anderen Ursache zugeschrieben als bei den Systemen $\mathrm{C}_{7} \mathrm{G}_{1}, \mathrm{C}_{8} \mathrm{G}_{1}$ und $\mathrm{C}_{9} \mathrm{G}_{1}-$ Simulationen der Mizellkinetik zeigen, dass bei 
kurzkettigen Systemen im Bereich der $c m c$ die Vereinfachung von Gl. (3.4) zu Gl (3.5), also die Annahme, dass nur vollständige Mizellen und Monomere interagieren können, nicht mehr gerechtfertigt ist [64]. Die Simulationsergebnisse zeigen nichtverschwindende Anteile von Relaxationsprozessen, deren Relaxationszeiten im Bereich der Hauptrelaxationszeit $\tau_{\text {Monol }}$ liegen, von denen speziell einer dem gefundenen zweiten Prozess zugeordnet werden könnte. Bei der experimentellen Überprüfung der Simulationsergebnisse in [64] wird der Monomeraustauschprozess mit einer verbreiterten Spektralfunktion, der empirischen Hill-Funktion, angepasst. Auch das Spektrum von $\mathrm{C}_{6} \mathrm{G}_{2}, 200 \mathrm{mmol} / \mathrm{L}$ kann mit einer solchen Funktion anstelle von zwei DebyeSpektralfunktionen zufriedenstellend angepasst werden. Da allerdings zum einen die vorliegende Anpassung etwas weniger Abweichungen von den Messpunkten aufweist und zum anderen eine genaue quantitative Analyse dieses Phänomens mangels Messpunkten nicht vorgenommen wird, werden hier nur die Parameter der Anpassung mit zwei Debye-Spektralfunktionen aufgeführt.

Bei den anderen Systemen muss das Auftreten eines zweiten Monomeraustauschprozesses vermutlich der speziellen Geometrie der jeweiligen Mizellen zugeschrieben werden. Um über diesen Zusammenhang qualitative oder sogar quantitative Aussagen treffen zu können, werden allerdings zunächst die Parameter des ,ersten' Monomeraustauschprozesses analysiert. Die Einteilung in einen ersten (ursprünglichen) und einen zweiten (zusätzlichen) Monomeraustauschprozess erfolgt dabei so, dass der Prozess, der bei allen Konzentrationen oberhalb der $c m c$ auftritt, als erster Prozess bezeichnet wird. Bei den Systemen $\mathrm{C}_{7} \mathrm{G}_{1}$ und $\mathrm{C}_{9} \mathrm{G}_{1}$, bei denen beide Prozesse dieses Kriterium erfüllen, wird jener Prozess als der ursprüngliche angenommen, dessen Amplitudenverlauf dem erwarteten gemäß Gl. (3.7) entspricht. In Abbildung 57 und Abbildung 58 sind der Verlauf der Relaxationsrate $\left(\tau_{\text {Monol }}\right)^{-1}$ und der Amplitude $A_{\text {Monol }}$ dieses (ersten) Monomeraustauschprozesses analog zu Abbildung 22 gegen die reduzierte Konzentration $X$ aufgetragen.

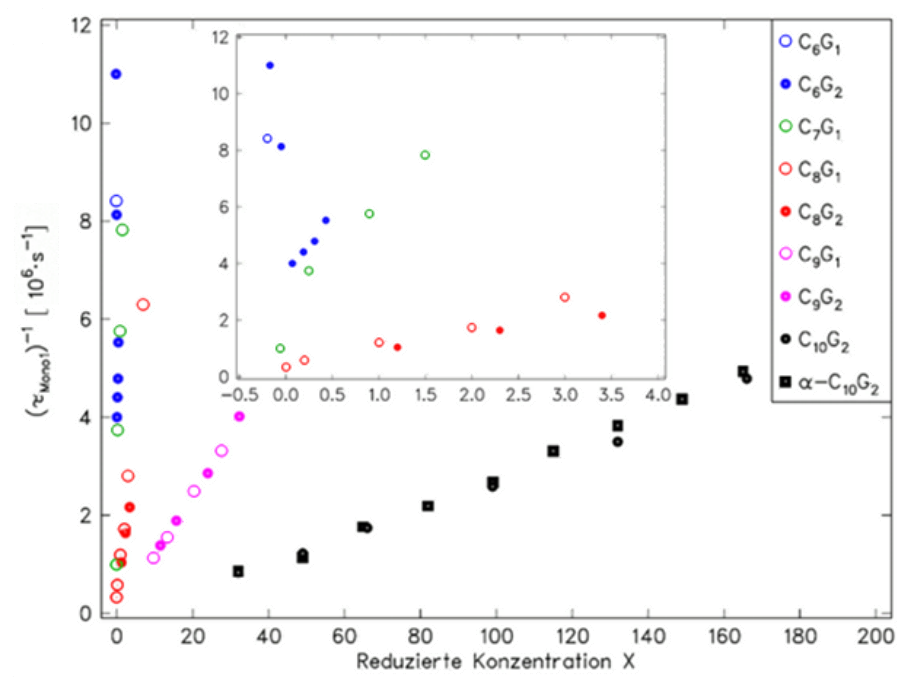

Abbildung 57: Verlauf der Relaxationsraten $\left(\tau_{\text {Mono1 }}\right)^{-1}$ des (ersten) Monomeraustauschprozesses über der reduzierten Konzentration $\boldsymbol{X}$ für die untersuchten Systeme. Im Inset ist der Bereich kleiner reduzierter Konzentrationen noch einmal gestreckter dargestellt. 
Der Verlauf der Relaxationsraten zeigt wie nach Gl. (3.6) zu erwarten eine lineare Abhängigkeit von der reduzierten Konzentration mit abnehmender Steigung bei zunehmender Alkylkettenlänge. Auffällig ist, dass sich die Relaxationsraten jener Systeme, bei denen sowohl das Glukosid als auch das entsprechende Maltosid untersucht wurden, als nahezu unabhängig von der Kopfgruppe erweisen. Da allerdings die Alkylkettenlänge der entscheidende Faktor in der Entropiebilanz des Monomeraustausches ist, ist ein solches Ergebnis auch zu erwarten.

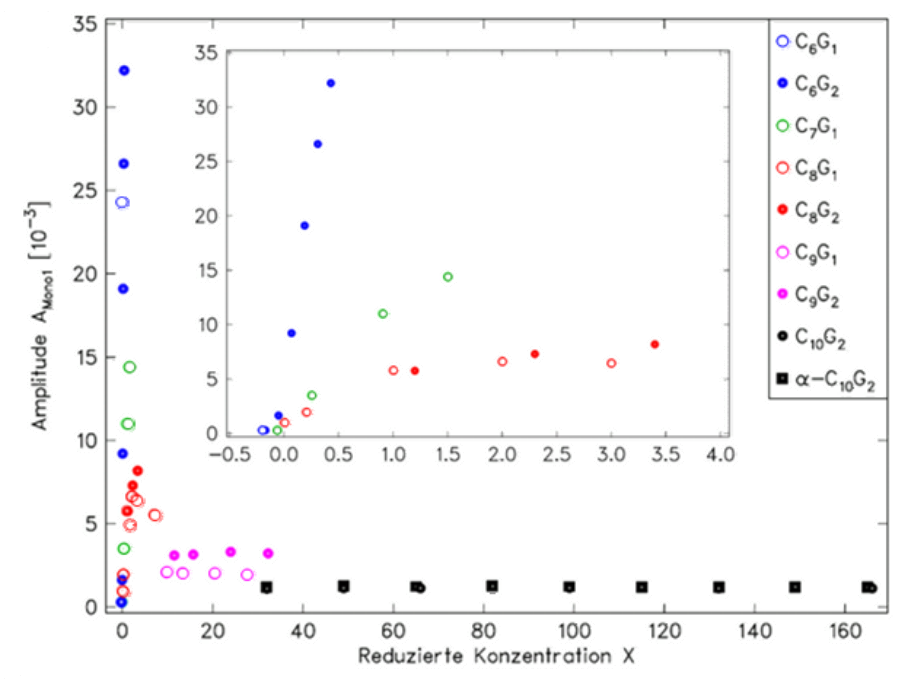

\begin{abstract}
Abbildung 58: Verlauf der Amplituden $A_{\text {Mono1 }}$ des (ersten) Monomeraustauschprozesses über der reduzierten Konzentration $\boldsymbol{X}$ für die untersuchten Systeme. Im Inset ist wiederum der Bereich kleiner reduzierter Konzentrationen gestreckter dargestellt.
\end{abstract}

Zur quantitativen Analyse wird nun eine lineare Regression gemäß Gl. (3.6) mit den Daten durchgeführt. Die resultierenden Parameter $k_{b} / \sigma^{2}$ (Achsenabschnitt) und $k_{b} / \bar{m}$ (Steigung) sind in Tabelle 5.1 aufgelistet. Zusätzlich sind die Werte für $\bar{m}$ (soweit bekannt) aus der Literatur aufgeführt und die damit berechneten Parameter $k_{b}$ und $\sigma$. Weiterhin lässt sich in [66] eine Näherung für die Hinreaktionskonstante $k_{f}$ finden: $k_{f} \approx k_{b} / c m c-$ die entsprechenden Werte sind ebenfalls in Tabelle 5.1 aufgelistet. Für das System $\mathrm{C}_{6} \mathrm{G}_{1}$ können die Berechnungen nicht durchgeführt werden, da beide untersuchten Konzentrationen unterhalb der $\mathrm{cmc}$ liegen.

Um die systemunabhängige Gültigkeit von Gl. (3.6) noch einmal besser zu veranschaulichen, ist in Abbildung 59 für alle relevanten Lösungen $\left(\left(\tau_{\text {Monol }}\right)^{-1}-k_{b} / \sigma^{2}\right) \cdot \bar{m} / k_{b}$ gegen $X$ aufgetragen. Das zu erwartende Zusammenfallen aller Werte auf eine Gerade ist offensichtlich für alle Systeme sehr gut erfüllt. Weiterhin kann für das System $\mathrm{C}_{8} \mathrm{G}_{1}$ ein Vergleich mit Literaturdaten vorgenommen werden. In [84] wurden Ultraschallabsorptionsspektren von $\mathrm{C}_{8} \mathrm{G}_{1}$-Lösungen vier verschiedener Konzentrationen $(40 \mathrm{mmol} / \mathrm{L}, 45 \mathrm{mmol} / \mathrm{l}, 60 \mathrm{mmol} / \mathrm{L}$ und $80 \mathrm{mmol} / \mathrm{L})$ in einem Frequenzbereich von $500 \mathrm{kHz}$ bis $150 \mathrm{MHz}$ aufgenommen und mit einer einzelnen DebyeSpektralfunktion angepasst. Trotz der unterschiedlichen Frequenzbereiche und der in dieser Arbeit zusätzlich angepassten Debye-Terme stimmen die resultierenden Parameter $\left(k_{b}=140 \cdot 10^{-6} \mathrm{~s}^{-1}, \sigma=15\right.$ und $\left.\Delta V / n_{C}=1.03\right)$ relativ gut mit den hier erhaltenen überein.

An den Daten der Tabelle 5.1 fällt auf, dass für die Systeme, bei denen nur weit oberhalb der $c m c$ gemessen werden konnte $\left(\mathrm{C}_{9} \mathrm{G}_{1}, \mathrm{C}_{9} \mathrm{G}_{2}, \mathrm{C}_{10} \mathrm{G}_{2}, \alpha-\mathrm{C}_{10} \mathrm{G}_{2}\right)$, aus der Anpassung ein negativer Achsenabschnitt resultiert. Dies 
ist natürlich physikalisch nicht sinnvoll, sondern beruht zum einen auf der relativ großen Extrapolationsdistanz für diese Systeme und zum anderen auf einer eventuell nicht ganz zutreffenden Angabe der $c m c$.

\begin{tabular}{|c|c|c|c|c|c|c|c|}
\hline Substanz & $\begin{array}{c}c m c \\
{[\mathrm{mmol} / \mathrm{L}]}\end{array}$ & $\bar{m}$ & $\begin{array}{c}k_{b} / \sigma^{2} \\
{\left[10^{6} \mathrm{~s}^{-1}\right]}\end{array}$ & $\begin{array}{c}k_{b} / \bar{m} \\
{\left[10^{6} \mathrm{~s}^{-1}\right]}\end{array}$ & $\begin{array}{c}k_{b} \\
{\left[10^{6} \mathrm{~s}^{-1}\right]}\end{array}$ & $\begin{array}{c}k_{f} \\
{\left[10^{6} \mathrm{~L} \cdot \mathrm{mol}^{-1} \cdot \mathrm{s}^{-1}\right]}\end{array}$ & $\sigma$ \\
\hline $\mathrm{C}_{6} \mathrm{G}_{1}$ & 250 & $55^{\#}$ & - & - & - & - & - \\
\hline $\mathrm{C}_{6} \mathrm{G}_{2}$ & 210 & $30^{\#}$ & 3.65 & 4.13 & 124 & 590 & 5.8 \\
\hline $\mathrm{C}_{7} \mathrm{G}_{1}$ & 70 & $63^{\#}$ & 2.88 & 3.26 & 205 & 2936 & 8.4 \\
\hline $\mathrm{C}_{8} \mathrm{G}_{1}$ & $25^{+}$ & $80^{+}$ & 0.29 & 0.85 & 68 & 2709 & 15.4 \\
\hline $\mathrm{C}_{8} \mathrm{G}_{2}$ & 19.5 & 47 & 0.44 & 0.51 & 24 & 1235 & 7.4 \\
\hline $\mathrm{C}_{9} \mathrm{G}_{1}$ & 6.5 & $95^{\#}$ & -0.07 & 0.12 & 12 & 1802 & - \\
\hline $\mathrm{C}_{9} \mathrm{G}_{2}$ & 6 & 55 & -0.09 & 0.13 & 7 & 1153 & - \\
\hline $\mathrm{C}_{10} \mathrm{G}_{2}$ & 1.8 & 69 & -0.2 & 0.03 & 2 & 1111 & - \\
\hline$\alpha-C_{10} G_{2}$ & $1.8^{*}$ & $69^{*}$ & -0.3 & 0.03 & 2 & 1199 & - \\
\hline
\end{tabular}

Tabelle 5.1: Parameter des Monomeraustauschprozesses, resultierend aus einer linearen Regression gemäß Gl. (3.6). " Mangels Literaturdaten wird angenommen, dass die Werte $c m c$ und $\bar{m}$ von $\alpha-\mathrm{C}_{10} \mathrm{G}_{2}$ und $\beta-C_{10} G_{2}$ gleich sind. ${ }^{+}$Aus den sehr unterschiedlichen Literaturdaten (siehe Kapitel 3.5) werden zunächst diese beiden benutzt. ${ }^{\#}$ Extra- bzw. interpolierte Werte.

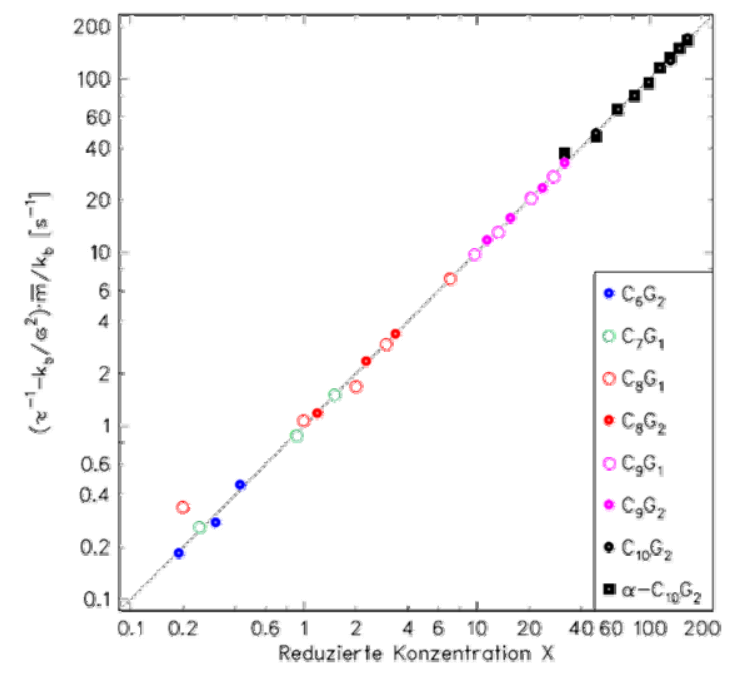

\section{Abbildung 59: Darstellung der linearen Abhängigkeit der Relaxationsrate des Monomeraustausch- prozesses $\left(\tau_{\text {Mono1 }}\right)^{-1}$ von der reduzierten Konzentration $X$.}

Die aus den Anpassungen resultierenden Werte für die Rückreaktionskonstante $k_{b}$ können nun mit Werten von anderen homologen Reihen verglichen werden. Dazu sind die $k_{b}$-Werte in Tabelle 5.2 zusammen mit denen einiger anderer Tenside aufgelistet. Aus den Werten werden zwei Trends sichtbar: Die Rückreaktionskonstante (ein Maß für das ,Bestreben eines Monomers aus einer Mizelle auszutreten') nimmt zum einen mit steigender Kettenlänge und zum anderen mit sinkender Hydrophilität der Kopfgruppe ab (ionische Kopfgruppen sind im Allgemeinen wesentlich hydrophiler als nichtionische). Diese beiden Trends sind plausibel, denn eine längere Alkylkette oder eine weniger hydrophile Kopfgruppe erhöhen den Entropiebeitrag, der letztlich zur Mizellbildung führt. Da auch die $c m c$ eine direkte Abhängigkeit von der Alkylkettenlänge und der Hydrophilität der Kopfgruppe zeigt, lässt sich auch ein direkter universeller Zusammenhang zwischen der Rückreaktionskonstante $k_{b}$ und 84 
der $c m c$ für die verschiedenen Systeme vermuten. In Abbildung 60 ist dieser Zusammenhang für die Werte aus dieser Arbeit und aus der Literatur graphisch dargestellt. Es zeigt sich, dass die Werte aus dieser Arbeit sehr gut in diesen universellen Zusammenhang hineinpassen.

\begin{tabular}{|c|c|c|c|c|c|}
\hline \multirow{2}{*}{ Substanzklasse } & \multicolumn{5}{|c|}{ Alkylkettenlänge $n_{C}=i$} \\
\hline & 6 & 7 & 8 & 9 & 10 \\
\hline \multicolumn{6}{|c|}{ nichtionische Kopfgruppe } \\
\hline $\mathrm{C}_{\mathrm{i}} \mathrm{E}_{3}$ & 589 & - & - & - & - \\
\hline $\mathrm{C}_{\mathrm{i}} \mathrm{E}_{4}$ & - & 330 & 12 & - & - \\
\hline $\mathrm{C}_{\mathrm{i}} \mathrm{E}_{5}$ & 550 & - & 50 & - & - \\
\hline $\mathrm{C}_{\mathrm{i}} \mathrm{G}_{1}$ & - & 205 & 68 & 12 & - \\
\hline $\mathrm{C}_{\mathrm{i}} \mathrm{G}_{2}$ & 124 & - & 24 & 7 & 2 \\
\hline \multicolumn{6}{|c|}{ anionische Kopfgruppe } \\
\hline $\mathrm{C}_{\mathrm{i}} \mathrm{ACl}$ & 8000 & 1890 & 820 & - & - \\
\hline $\mathrm{C}_{\mathrm{i}} \mathrm{TABr}$ & - & - & 1010 & - & 170 \\
\hline \multicolumn{6}{|c|}{ kationische Kopfgruppe } \\
\hline $\mathrm{C}_{\mathrm{i}} \mathrm{NaS}$ & 1300 & 730 & 100 & - & - \\
\hline
\end{tabular}

Tabelle 5.2: Rückreaktionskonstante $\boldsymbol{k}_{b}$ für einige Tenside in Abhängigkeit von der Kopfgruppe und der Alkylkettenlänge. $\mathrm{C}_{\mathrm{i}} \mathrm{E}_{\mathrm{j}}$ steht für Poly(ethylenglykol)monoalkylether [68], [111], [112], $\mathrm{C}_{\mathrm{i}} \mathrm{ACl}$ für Alkylammoniumchloride [29], $\mathrm{C}_{\mathrm{i}} \mathrm{NaS}$ für Natriumalkylsulfate [109] und $\mathrm{C}_{\mathrm{i}} \mathrm{TABr}$ für Alkyltrimethylammoniumbromide [110]. Alle Werte in $10^{6} \mathrm{~s}^{-1}$.

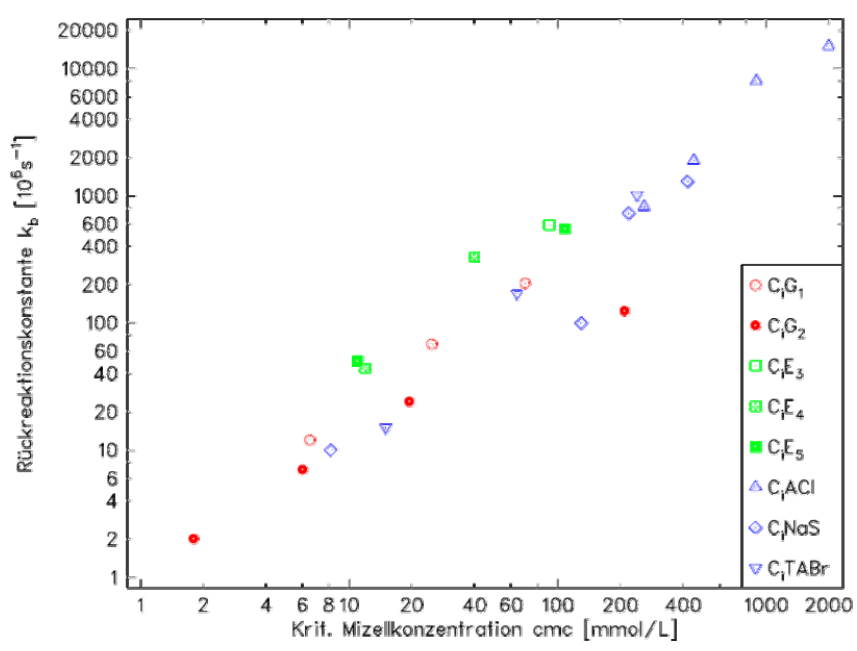

Abbildung 60: Abhängigkeit der Rückreaktionskonstante $k_{b}$ des Monomeraustauschprozesses von der $c m c$ für die untersuchten Alkylglykoside und andere Tensid-Systeme. Abkürzungen und Literaturquellen wie in Tabelle 5.2.

Zur Auswertung der Amplituden des Monomeraustauschprozesses wird aus den einzelnen Amplituden gemäß Gl. (3.7) das Reaktionsvolumen $\Delta V$ berechnet. Für die Systeme $\mathrm{C}_{6} \mathrm{G}_{2}, \mathrm{C}_{7} \mathrm{G}_{1}, \mathrm{C}_{8} \mathrm{G}_{1}$ und $\mathrm{C}_{8} \mathrm{G}_{2}$ könnten dabei die Werte für $\sigma$ aus Tabelle 5.1 benutzt werden. Da diese aber zum einen mit einer großen Unsicherheit behaftet sind und hier zum anderen für die Amplitudenauswertung keine aus der Relaxationszeitenauswertung erhaltenen Werte benutzt werden sollen um eine mögliche Fortpflanzung systematischer Fehler zu vermeiden, wird zunächst für alle Systeme angenommen, dass das Amplitudenplateau in Gl. (3.7) bzw. Abbildung 22 erreicht ist $\left(A_{\text {Mo- }}\right.$ 
${ }_{\text {nol }}=\pi \cdot(\Delta V)^{2} \cdot c m c \cdot c_{S}^{2} \cdot \rho \cdot R^{-1} \cdot T^{-1}$ für $\left.X » 1\right)$. Da davon auszugehen ist, dass das Reaktionsvolumen $\Delta V$ linear abhängig von der Alkylkettenlänge $n_{C}$ ist, ist in Abbildung 61 das erhaltene Reaktionsvolumen pro $\mathrm{CH}_{2}$-Gruppe gegen die reduzierte Konzentration $X$ aufgetragen.

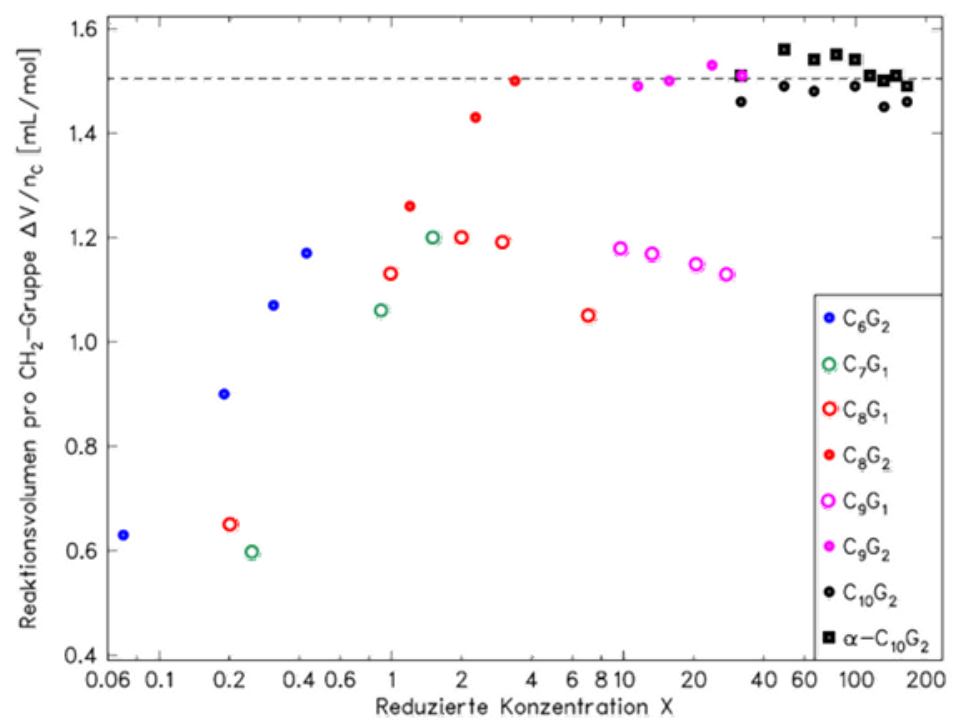

\section{Abbildung 61: Aus den Amplituden des Monomeraustauschprozess errechnetes Reaktionsvolumen pro $\mathrm{CH}_{2}$-Gruppe in Abhängigkeit von der reduzierten Konzentration $X$.}

In der obigen Auftragung wird deutlich, dass für die Systeme $C_{9} G_{2}, C_{10} G_{2}$ und $\alpha-C_{10} G_{2}$ offenbar ein Wert von 1.5 $\mathrm{mL} / \mathrm{mol}$ pro $\mathrm{CH}_{2}$-Gruppe die Amplituden sehr gut beschreibt. Dieser Wert scheint realistisch, verglichen mit einem Wert von $1.61 \mathrm{~mL} / \mathrm{mol}$ pro $\mathrm{CH}_{2}$-Gruppe, den Telgmann für 11 verschiedene Poly(ethylenglykol)monoalkylether $\left(\mathrm{C}_{\mathrm{i}} \mathrm{E}_{\mathrm{j}}\right)$ findet [68]. Weiterhin wird deutlich, dass für die Systeme $\mathrm{C}_{6} \mathrm{G}_{2}, \mathrm{C}_{7} \mathrm{G}_{1}$ und $\mathrm{C}_{8} \mathrm{G}_{2}$ das Amplitudenplateau noch nicht, bzw. bei letzterem gerade eben mit der höchsten untersuchten Konzentration $(c=100 \mathrm{mmol} / \mathrm{L}, X \approx 3.5)$ erreicht wird. Bemerkenswert sind das Abknicken der Werte für das System $\mathrm{C}_{8} \mathrm{G}_{1}$ und die deutlich niedrigeren Werte für das System $\mathrm{C}_{9} \mathrm{G}_{1}$. Beide Verläufe lassen sich allerdings plausibel dadurch erklären, dass für diese Systeme das Auftreten eines zweiten Monomeraustauschprozesses beobachtet wird. Für eine zunächst grobe quantitative Analyse dieses Zusammenhangs kann für die Systeme $C_{7} G_{1}, C_{8} G_{1}$ und $\mathrm{C}_{9} \mathrm{G}_{1}$ das Reaktionsvolumen aus der Summe der beiden Monomeraustauschprozess-Amplituden berechnet werden. In allen drei Fällen liegen die Werte dann zwar etwas höher als in Abbildung 61, aber maximal bei 1.3 $\mathrm{mL} / \mathrm{mol}$ pro $\mathrm{CH}_{2}$-Gruppe, also immer noch recht deutlich unter dem erwarteten Wert. Dieses Ergebnis deutet darauf hin, dass entweder eine genauere Analyse des Einflusses der Mizellgeometrie auf die Amplitude des Monomeraustauschprozesses notwendig ist oder dass die Alkylkette der Tenside in einer nicht kugelförmigen Mizelle schlechter vom umgebenden Wasser abgeschirmt wird als in einer kugelförmigen Mizelle. Da vermutlich beide Aussagen zutreffen, wird zum Vergleich eine alternative Berechnung des Reaktionsvolumens herangezogen: Aus den Dichtedaten der Lösungen lassen sich die scheinbaren molaren Volumina $V_{\text {schein }}$ der gelösten Moleküle, also der Tenside, bestimmen:

$$
V_{\text {schein }}=\frac{\left(\rho_{\text {Wasser }}-\rho_{\text {Lsg }}\right)}{\hat{c} \cdot \rho_{\text {Wasser }} \cdot \rho_{L s g}}+\frac{M W}{\rho_{L s g}}
$$


Hierbei ist $\hat{c}$ die Molalität der Lösung: $\hat{c}=n_{\text {Subst }} / m_{\text {Wasser }}$. Der Begriff, scheinbar' deutet darauf hin, dass bei dieser Berechnung das Volumen eines gelösten Moleküls inklusive aller Volumen-Wechselwirkungen mit dem umgebenden Wasser bestimmt wird. Bei Tensidlösungen kann aus der Konzentrationsabhängigkeit dieser Größe das Volumen der Hydrathüllenänderung bestimmt werden. So beinhaltet sie im monomeren Zustand $(c \ll c m c)$ das Volumen des Monomers und das Reaktionsvolumen, während der Anteil der Hydrathüllenwechselwirkung der Alkylkette im mizellaren Zustand ( $(\gg c m c$, unter Vernachlässigung der auch dort noch vorhandenen Monomere) bei vollständiger Abschirmung der Alkylkette vom umgebenden Wasser nicht mehr enthalten ist. Daher ergibt sich für die Differenz $V_{\text {schein }}(c » c m c)-V_{\text {schein }}(c \ll c m c)=\Delta V$. Dass diese Bestimmung des Reaktionsvolumens und die Bestimmung mithilfe der Ultraschallabsorptionsspektroskopie im Rahmen der Fehler gleichgroße Werte liefert, ist experimentell für verschiedene nichtionische Tensidsysteme überprüft worden [111]-[114]. Da in dieser Arbeit für keines der Systeme Dichtemessungen sowohl deutlich unterhalb als auch deutlich oberhalb der $c m c$ vorgenommen werden konnten, wird hier folgendermaßen vorgegangen: Das scheinbare molare Volumen wird für $\mathrm{C}_{10} \mathrm{G}_{2}$ aus den Daten der $250 \mathrm{mmol} / \mathrm{L}-$ Lösung $\left(c \gg c m c \rightarrow \Delta V \ll V_{\text {schein }}\right)$ bestimmt und fiktiv in zwei Anteile aufgeteilt: $V_{\text {schein }}=V_{\mathrm{C} 10}+V_{\mathrm{G} 2}$. Zudem wird das scheinbare molare Volumen von Maltose für alle untersuchten Konzentrationen berechnet und gemittelt. Es zeigt sich, dass die so erhaltenen Werte $V_{\text {schein }}\left(\mathrm{C}_{10} \mathrm{G}_{2}, 250\right.$ $\mathrm{mmol} / \mathrm{L})=386 \mathrm{~mL} / \mathrm{mol}$ und $V_{\text {schein }}($ Maltose $)=210 \mathrm{~mL} / \mathrm{mol}$ sich ziemlich genau um das nach Gl. (3.8) berechenbare Volumen der Decylkette $V_{\mathrm{C} 10}=178 \mathrm{~mL} / \mathrm{mol}$ unterscheiden: $386 \mathrm{~mL} / \mathrm{mol}-210 \mathrm{~mL} / \mathrm{mol}=176 \mathrm{~mL} / \mathrm{mol}$. Das scheinbare Volumen des Maltosekopfes des Tensids ist also gleich dem scheinbaren Volumen von reiner Maltose: $V_{\text {schein }}($ Maltose $) \approx V_{\mathrm{G} 2}$. Dementsprechend lässt sich vermuten, dass auch das scheinbare Volumen eines Glukosekopfes gleich dem scheinbaren Volumen reiner Glukose ist. Daher wird jenes für alle Konzentrationen berechnet - der gemittelte Wert beträgt dann $V_{\text {schein }}($ Glukose $)=111 \mathrm{~mL} / \mathrm{mol}$. Nun kann für alle Alkylglykoside das scheinbare Volumen im monomeren Zustand berechnet werden, unter der Annahme, dass das Reaktionsvolumen in $\mathrm{mL} / \mathrm{mol}$ entsprechend den Ergebnissen aus der Ultraschallabsorptionsspektroskopie gerade 1.5 mal die Alkylkettenlänge $n_{\mathrm{C}}$ ist: $V_{\text {schein }}{ }^{\text {Mono }}=V_{\mathrm{Ci}}+V_{\mathrm{G} 1}+\Delta V=V_{\mathrm{Ci}}+111 \mathrm{~mL} / \mathrm{mol}+1.5 \mathrm{~mL} / \mathrm{mol} \cdot n_{\mathrm{C}}$ für die Alkylglukoside bzw. $V_{\text {schein }}{ }^{\text {Mono }}=V_{\mathrm{Ci}}+V_{\mathrm{G} 2}+\Delta V=V_{\mathrm{Ci}}+210 \mathrm{~mL} / \mathrm{mol}+1.5 \mathrm{~mL} / \mathrm{mol} \cdot n_{\mathrm{C}}$ für die Alkylmaltoside. Entsprechend gilt für das theoretisch berechenbare scheinbare Volumen im mizellaren Zustand $V_{\text {schein }}{ }^{M i z}=V_{\mathrm{Ci}}+V_{\mathrm{G} 1}=V_{\mathrm{Ci}}+111 \mathrm{~mL} / \mathrm{mol}$ bzw. $V_{\text {schein }}{ }^{M i z}=V_{\mathrm{Ci}}+V_{\mathrm{G} 2}=V_{\mathrm{Ci}}+210 \mathrm{~mL} / \mathrm{mol}$. Mit diesen beiden Größen kann nun ein theoretischer konzentrationsabhängiger Verlauf des scheinbaren Volumens für den Fall vollständiger Abschirmung der Alkylketten in den Mizellen berechnet werden: $V_{\text {schein }}\left(c_{\text {ges }}\right)=\left(c_{\text {Mono }} \cdot V_{\text {schein }}{ }^{\text {Mono }}+c_{\text {Miz }} \cdot V_{\text {schein }}{ }^{\text {Miz }}\right) / c_{\text {ges }}$. Diese Verläufe sind zusammen mit den aus den Dichtedaten berechneten Werten für die drei Systeme $C_{7} G_{1}, C_{8} G_{1}$ und $C_{9} G_{1}$ in Abbildung 62 aufgetragen. 


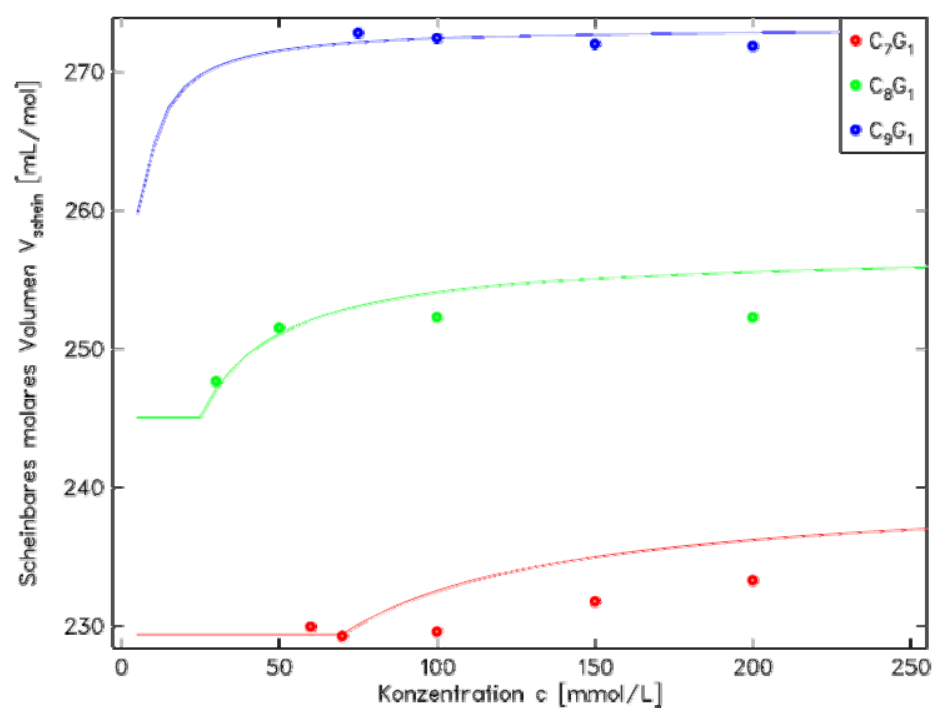

Abbildung 62: Scheinbares molares Volumen in Abhängigkeit von der Konzentration für die Systeme $\mathbf{C}_{7} \mathbf{G}_{1}, \mathbf{C}_{8} \mathbf{G}_{1}$ und $\mathbf{C}_{9} \mathbf{G}_{1}$. Die Punkte sind gemäß Gl. (5.1) aus den Dichtedaten berechnet, die Linien geben den theoretisch berechneten Verlauf unter Annahme vollständiger Abschirmung der Alkylketten im mizellaren Zustand wieder (siehe Text).

Setzt man die Gültigkeit des Modells additiver scheinbarer Volumina der verschiedenen Molekülteile voraus, kann obige Abbildung als Indiz dafür angesehen werden, dass bei den Systemen, die einen zweiten Monomeraustauschprozess aufweisen, vermutlich keine vollständige Abschirmung der Alkylkette in der Mizelle erfolgt. Lediglich beim System $\mathrm{C}_{9} \mathrm{G}_{1}$ weisen die Messpunkte keine deutliche Abweichung von der theoretischen Berechnung auf. Für eine weitere qualitative und quantitative Behandlung des Zusammenhangs zwischen den Parametern des Monomeraustauschprozesses und der Mizellgeometrie soll nun der Verlauf der zweiten Relaxationszeit, bzw. der Relaxationsrate, über der reduzierten Konzentration betrachtet werden. In Abbildung 63 ist dieser Verlauf für die Systeme $\mathrm{C}_{7} \mathrm{G}_{1}, \mathrm{C}_{8} \mathrm{G}_{1}$ und $\mathrm{C}_{9} \mathrm{G}_{1}$ aufgetragen.

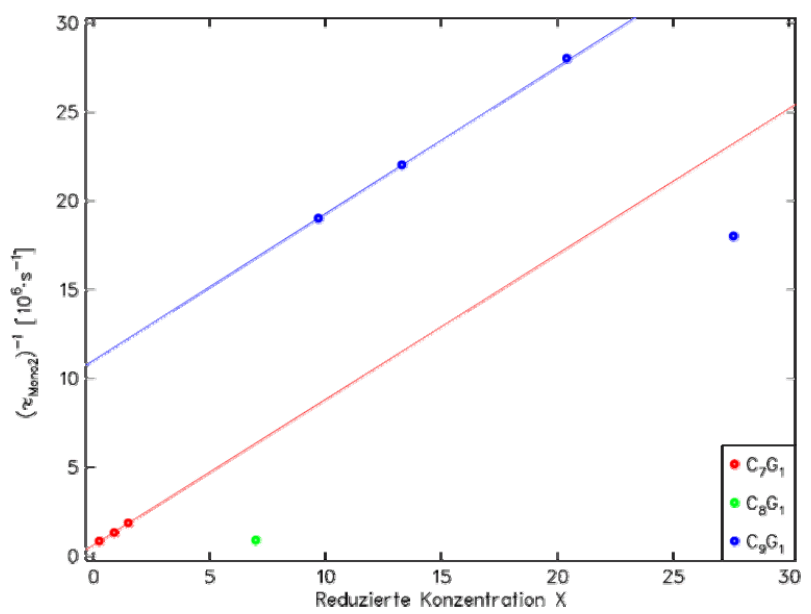

Abbildung 63: Verlauf der Relaxationsraten $\left(\tau_{\mathrm{Mon} 02}\right)^{-1}$ des zweiten Monomeraustauschprozesses über der reduzierten Konzentration $X$. Für $C_{7} G_{1}$ und $C_{9} G_{1}$ ist bereits eine lineare Regression eingetragen, der abweichende Punkt des Systems $\mathrm{C}_{9} \mathrm{G}_{1}$ wurde bei der Anpassung nicht berücksichtigt.

Aus der Auftragung wird ersichtlich, dass auch die Relaxationsraten des zweiten Monomeraustauschprozesses linear von der reduzierten Konzentration abhängen (die Abweichung des am weitesten rechts gelegenen Punktes 
wird durch die reziproke Darstellung verstärkt - eine Anpassung des entsprechenden Spektrums mit einer besser zur linearen Abhängigkeit in Abbildung 63 passenden Relaxationszeit ist ohne deutliche Verschlechterung der Beschreibung des Spektrums und ohne deutlichen Einfluss auf die anderen Spektralparameter möglich). Auf eine lineare Regression muss für das System $\mathrm{C}_{8} \mathrm{G}_{1}$ mangels Punkten verzichtet werden. Allerdings erklärt der vorhandene Punkt möglicherweise die Tatsache, dass bei nur einer Konzentration der $\mathrm{C}_{8} \mathrm{G}_{1}$-Lösungen ein zweiter Monomeraustauschprozess gefunden wurde. Denn die Relaxationszeiten für die restlichen untersuchten $C_{8} G_{1}$ Lösungen liegen unterhalb des messbaren Frequenzbereiches, wenn der Verlauf von $\left(\tau_{\text {Mono2 }}\right)^{-1}$ über $X$ bei diesem System eine ähnliche Steigung aufweist wie für die beiden anderen Systeme. Für jene ergeben sich durch lineare Regression folgende Werte:

\begin{tabular}{l|r|r|r|r|r|r|r}
\hline \hline Substanz & $\begin{array}{c}c m c \\
{[\mathrm{mmol} / \mathrm{L}]}\end{array}$ & $\bar{m}$ & $\begin{array}{c}k_{b} / \sigma^{2} \\
{\left[10^{6} \mathrm{~s}^{-1}\right]}\end{array}$ & $\begin{array}{c}k_{b} / \bar{m} \\
{\left[10^{6} \mathrm{~s}^{-1}\right]}\end{array}$ & $\begin{array}{c}k_{b} \\
{\left[10^{6} \mathrm{~s}^{-1}\right]}\end{array}$ & $\begin{array}{c}k_{f} \\
{\left[10^{6} \mathrm{~L} \cdot \mathrm{mol}^{-1} \cdot \mathrm{s}^{-1}\right]}\end{array}$ & $\sigma$ \\
\hline $\mathrm{C}_{7} \mathrm{G}_{1}$ & 70 & $63^{\#}$ & 0.61 & 0.82 & 52 & 737 & 9.2 \\
$\mathrm{C}_{9} \mathrm{G}_{1}$ & 6.5 & $95^{\#}$ & 11 & 0.82 & 78 & 12070 & 2.7 \\
\hline \hline
\end{tabular}

Tabelle 5.3: Auflistung der Parameter des zweiten Monomeraustauschprozesses, resultierend aus einer linearen Regression gemäß Gl. (3.6). "Extra- bzw. interpolierte Werte (siehe Tabelle 5.1).

Hier stellt sich die Frage, ob das Einsetzen der Literaturwerte für $\bar{m}$, die unter Annahme einer Kugelgeometrie bestimmt wurden, noch sinnvoll ist, wenn man davon ausgeht, dass dieser zweite Monomeraustauschprozess durch eine veränderte Geometrie verursacht wird. Um diese Überlegung vertiefen zu können, werden im Folgenden zwei unterschiedliche Modelle für die Konzentrationsabhängigkeit von Mizellgröße und -geometrie vorgestellt, um anschließend beurteilen zu können, welches der beiden Modelle durch die vorliegenden Messdaten unterstützt wird.

Modell 1 - Kontinuierliches Mizellwachstum: In diesem Modell geht man davon aus, dass die Mizellen bei $c \approx c m c$ kugelförmig sind und bei Erhöhung der Tensidkonzentration kontinuierlich anwachsen. Ein solches Anwachsen kann aus geometrischen Gründen nur so erfolgen, dass sich die Form der Mizellen zu Ellipsoiden, Scheibchen oder Stäbchen (siehe Abbildung 24) verändert und die Exzentrität (bei Ellipsoiden), der Durchmesser (bei Scheibchen) oder die Länge (bei Stäbchen) mit steigender Tensidkonzentration zunimmt. Das bedeutet zum einen, dass die mittlere Aggregationszahl $\bar{m}$ (und evtl. auch die Verteilungsbreite $\sigma$ ) keine Konstante ist, sondern eine (monoton steigende) Funktion der Tensidkonzentration, während zum anderen die Gesamtanzahl an Mizellen nicht mehr mit der Tensidkonzentration ansteigt (oder zumindest nicht mehr linear wie beim Fall kugelförmiger Mizellen).

Modell 2 - Koexistenz zweier Mizellarten: In diesem Modell existieren in der Lösung sowohl kugelförmige Mizellen als auch andere Mizellen - letztere entweder bereits direkt oberhalb der $c m c$ oder erst ab einer bestimmten Konzentration, einer Art ,zweiter $c m c$ '. Damit ist die Definition einer einzelnen mittleren Aggregationszahl $\bar{m}$ und einer einzelnen Verteilungsbreite $\sigma$ nicht mehr sinnvoll, sondern das System muss durch jeweils zwei Größen $\left(\bar{m}_{1}\right.$ und $\bar{m}_{2}$ bzw. $\sigma_{1}$ und $\left.\sigma_{2}\right)$ beschrieben werden. Diese werden als konzentrationsunabhängig angenommen, obwohl auch eine Kombination mit Modell 1, also die Koexistenz zweier Mizellarten, von denen eine oder beide mit zunehmender Tensidkonzentration wachsen, nicht per se ausgeschlossen werden kann. Ent- 
scheidend ist bei diesem Modell, dass bei vielen Untersuchungen an einem solchen System vermutlich ein Mittelwert der beiden jeweiligen Größen aufgenommen wird.

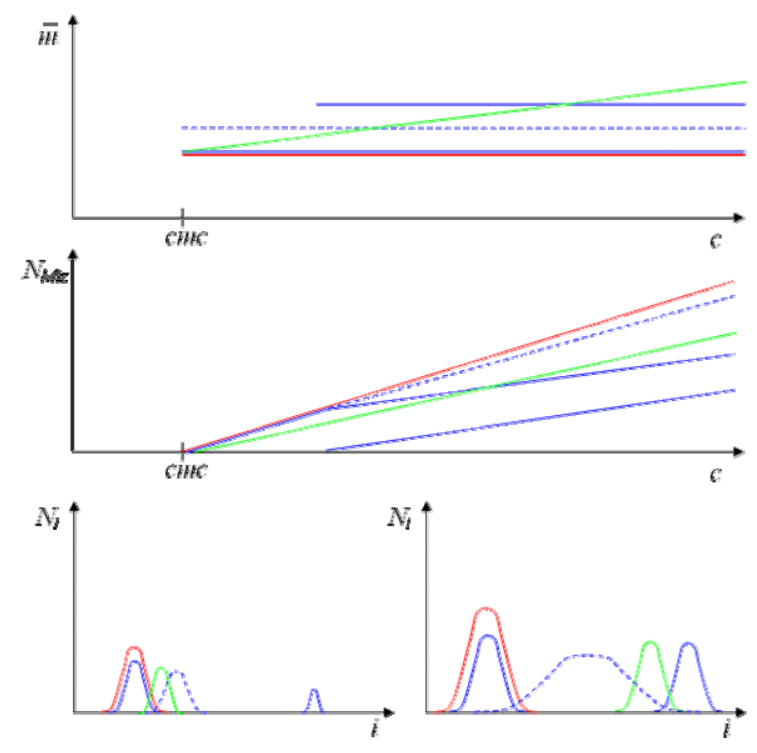

\begin{abstract}
Abbildung 64: Schematische Darstellung des Verlaufes der mittleren Aggregationszahl $\bar{m}$ (oben) und der Anzahl an Mizellen $N_{M i z}$ (Mitte) als Funktion der Tensidkonzentration sowie einer möglichen Aggregatgrößenverteilung bei einer Konzentration knapp oberhalb der $c m c$ (unten links) bzw. deutlich oberhalb der $c m c$ (unten rechts) für die Modelle ausschließlich kugelförmiger Mizellen (rot), kontinuierlich anwachsender Mizellen (Modell 1, grün) und zweier koexistenter Mizellarten (Modell 2, blau). Für letzteren Fall ist jeweils durch gestrichelte Linien ein Mittelwert als möglicher Messwert angedeutet.
\end{abstract}

Während bei Modell 2 der erste Monomeraustauschprozess offensichtlich den Monomeraustausch der kugelförmigen Mizellen beschreibt und der zweite Prozess den Austausch andersförmiger Mizellen, ist im Fall von Modell 1 davon auszugehen, dass der erste Monomeraustauschprozess den Eintritt/Austritt eines Monomers an einem kugelförmigen Teil der Mizelle beschreibt und der zweite Monomeraustauschprozess den Eintritt/Austritt an einem nicht kugelförmigen Teil. Das Volumen pro Molekül in einer kugelförmigen Mizelle oder einem kugelförmigen Mizellteil hat die Form eines Kegels und zum Beispiel bei einem zylindrischen Mizellteil die Form eines Tortenstückes oder Prismas (siehe Abbildung 65). Es erscheint plausibel, dass für diese unterschiedlichen geometrischen Gegebenheiten unterschiedliche Reaktionskonstanten und Reaktionsvolumina resultieren und somit zwei diskrete unterschiedliche Monomeraustauschprozesse.
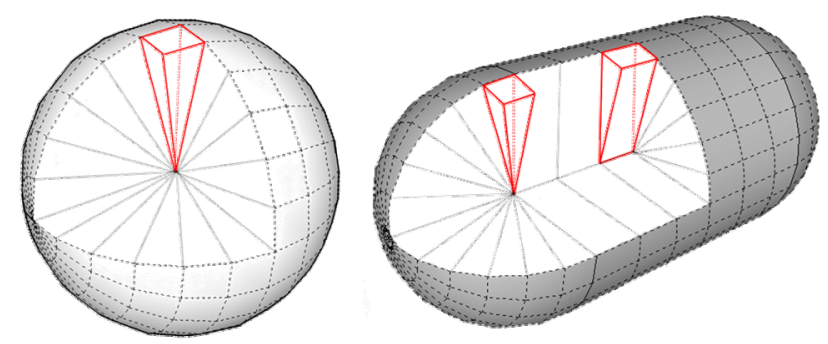

Abbildung 65: Schematische Darstellung des Volumens eines Moleküls in einer kugelförmigen (links) und einer stäbchenförmigen (rechts) Mizelle. 
Eine detaillierte theoretische Beschreibung der zwei unterschiedlichen Monomeraustauschprozesse wäre für beide Modelle extrem aufwändig, da für die Herleitung eines Ausdruckes analog zu Gl. (3.6) und Gl. (3.7) für die Relaxationszeit und die Amplitude die Betrachtung zweier Sätze gekoppelter Reaktionsgleichungen mit teilweise voneinander abhängigen Unbekannten $\left(\bar{m}_{1}, \bar{m}_{2}, \sigma_{1}, \sigma_{2}\right)$ vonnöten wäre (siehe [66]). Daher soll hier nur beurteilt werden, ob die experimentellen Ergebnisse eines der beiden Modelle ausschließen können.

Zunächst erscheint es für beide Modelle widersprüchlich, dass die Verläufe der Relaxationsraten des ersten Monomeraustauschprozesses und die daraus resultierenden Rückreaktionskonstanten $k_{b}$ so gut zu den Daten anderer Systeme passen, für die keine Hinweise auf nicht kugelförmige Mizellen existieren (siehe Abbildung 60). Insbesondere wurden für die Berechnung von $k_{b}$ aus der Literatur entnommene oder sogar extra-/interpolierte konzentrationsunabhängige Werte für die mittlere Aggregationszahl $\bar{m}$ benutzt. Bei Modell 1 erwartet man hingegen eine Konzentrationsabhängigkeit von $\bar{m}$ und bei Modell 2 würden die Literaturdaten sicherlich eher einem Mittelwert aus $\bar{m}_{1}$ und $\bar{m}_{2}$ entsprechen als dem hier benötigten Wert für die kugelförmigen Mizellen. Dass die Rückreaktionskonstanten dennoch so gut in den Kontext anderer Tenside passen, liegt daran, dass die Größenordnung der Rückreaktionskonstante sich bei leichter Variation von $\bar{m}$ nicht ändert. Dementsprechend wurde auch für die Auswertung des Systems $\mathrm{C}_{8} \mathrm{G}_{1}$ relativ willkürlich eine mittlere Aggregationszahl von $\bar{m}=80$ aus dem weiten Bereich der Literaturdaten $(\bar{m}=24$ bis $\bar{m}=105$, siehe Kapitel 3.5) benutzt.

Entscheidender ist hier vielmehr das experimentelle Resultat, dass die Relaxationsraten sowohl des ersten als auch des zweiten Monomeraustauschprozesses mit der Konzentration ansteigen (siehe Abbildung 57 und Abbildung 63). Dieser Anstieg reflektiert die ansteigende Begegnungswahrscheinlichkeit zwischen Mizellen und Monomeren durch die zunehmende Anzahl an Mizellen. Dementsprechend kann für Modell 1 ausgeschlossen werden, dass bei Erhöhung der Konzentration ein reines Wachstum der Mizellen stattfindet und für Modell 2 , dass eine konstante Konzentration an kugelförmigen Mizellen vorliegt. Letztendlich kann allerdings über diese Einschränkung hinaus aufgrund der Messdaten keines der beiden vorgestellten Modelle ausgeschlossen werden. Hierfür wären entweder aufwändige neue theoretische Beschreibungen notwendig oder sichere Erkenntnisse über die in den Lösungen vorliegenden Mizellformen aus anderen Messungen.

Für zukünftige Untersuchungen zu diesem Thema sollen hier noch einige weitere Ergebnisse aus den vorliegenden Messungen erwähnt werden, die noch nicht eindeutig erklärt werden können:

- Der zweite Monomeraustauschprozess tritt in den vorliegenden Spektren nur bei den Alkylglykosiden mit einer Glukose-Kopfgruppe auf und nicht bei jenen mit einer Maltose-Kopfgruppe. Dies bedeutet entweder, dass aus der Geometrie von Alkylmaltosid-Mizellen kein zusätzlicher Monomeraustausch resultiert oder dass ein entsprechender Prozess deutlich langsamer verläuft $\left(\tau_{\text {Mono2 } 2} 10^{-6} \mathrm{~s}\right)$ und daher außerhalb des in dieser Arbeit zugänglichen Messbereiches liegt. Betrachtet man die für den Packungsparameter $P$ und damit für die Mizellform relevanten Parameter Alkylkettenvolumen $V_{C}$ und -länge $l_{C}$, sowie die Querschnittsfläche der Kopfgruppe $A_{0}$ (siehe Gl. (3.9)), so sind die beiden ersteren für ein Alkylglukosid und das entsprechende Alkylmaltosid sicherlich gleich, während letzteres vermutlich für die Maltoseköpfe deutlich größer ist. Dementsprechend ist für Alkylglukoside ein größerer Packungsparameter $P$ zu erwarten als für Alkylmaltoside, was nach Tabelle 3.1 mit einer stärkeren Abweichung von der Kugelform korrespondieren würde. 
- Die Amplitude des zweiten Monomeraustauschprozesses bei dem System $\mathrm{C}_{7} \mathrm{G}_{1}$ zeigt ein untypisches Verhalten, da sie bei zunehmender Konzentration signifikant abnimmt (siehe Tabelle 4.4). Ein solches Verhalten kann nur mit einer Abnahme der an dieser spezifischen Reaktion beteiligten Reaktionspartner oder einer Abnahme des Reaktionsvolumens erklärt werden. Während ersteres einen Widerspruch zu der ansteigenden Relaxationsrate bedeuten würde, könnte eine Abnahme des Reaktionsvolumens dadurch erklärt werden, dass der Messwert einen Mittelwert aus einem kleineren und einem größeren Reaktionsvolumen darstellt und bei zunehmender Konzentration der Anteil des kleineren Reaktionsvolumens zunimmt. Beim System $\mathrm{C}_{9} \mathrm{G}_{1}$ hingegen nimmt die Amplitude des ersten Monomeraustauschprozesses leicht ab (siehe Tabelle 4.7) - allerdings liegt diese Abnahme im Bereich des Messfehlers und sollte daher nicht überbewertet werden.

- Kleinwinkelröntgenstreu-Messungen an den Systemen $\mathrm{C}_{7} \mathrm{G}_{1}, \mathrm{C}_{8} \mathrm{G}_{1}$ und $\mathrm{C}_{9} \mathrm{G}_{1}$ ergeben für zylindrische Mizellen einen Radius von ca. $1.4 \mathrm{~nm}$ für alle drei Systeme, aber sehr stark mit der Alkylkettenlänge ansteigende Zylinderlängen von $1.0 \mathrm{~nm}\left(\mathrm{C}_{7} \mathrm{G}_{1}\right), 3.5 \mathrm{~nm}\left(\mathrm{C}_{8} \mathrm{G}_{1}\right)$ und $160 \mathrm{~nm}\left(\mathrm{C}_{9} \mathrm{G}_{1}\right)$ [79]. Wegen dieser extrem unterschiedlichen Geometrien ist möglicherweise für die Systeme $C_{7} G_{1}$ und $C_{9} G_{1}$ eine einheitliche Zuordnung der beiden gefundenen Monomeraustauschprozesse gar nicht sinnvoll möglich.

Abschließend soll hier noch erörtert werden, warum eine Zuordnung des zweiten tieffrequenten Relaxationsprozesses zu anderen in Tensidsystemen auftretenden Vorgängen ausgeschlossen wird. Die bereits beim System $\mathrm{C}_{6} \mathrm{G}_{2}$ erwähnte Erweiterung des Modells der schrittweisen Aggregation bzw. die daraus resultierenden Konsequenzen für die Ultraschallabsorptionsspektren [64] spielen im Allgemeinen nur bei kurzkettigen Tensiden und im Bereich der $\mathrm{cmc}$ eine signifikante Rolle und kommen damit als Ursache für die vorliegenden Ergebnisse aus Messungen deutlich oberhalb der $c m c$ nicht in Betracht. Ein zweites in Tensidlösungen auftretendes Relaxationsphänomen ist die begrenzte radiale Diffusion, also das teilweise Austreten eines Monomers aus einer Mizelle. Für diesen Prozess wird allerdings von Aniansson eine konzentrationsunabhängige Relaxationszeit und eine mit der Konzentration ansteigende Amplitude vorhergesagt [9], was hier nicht vorliegt. Experimentelle Ergebnisse bestätigen diese Vorhersagen für andere Tenside [29]. Mit der gleichen Begründung kann auch die Relaxation durch Kinkenbildung der Alkylketten innerhalb der Mizelle ausgeschlossen werden - zudem liegt deren Relaxationszeit für ähnlich lange Alkylketten in einem anderen Bereich als die hier betrachteten $\left(\tau_{\text {Kinkenbildung }} \approx 0.2 \mathrm{~ns}\right.$ für $\mathrm{C}_{5} \mathrm{ACl}$ und $\left.\mathrm{C}_{8} \mathrm{ACl}[29]\right)$. 


\subsection{Die Rotation um die glykosidischen Bindungswinkel ( $\varphi-$, $\Psi$-Rotation)}

Der mittelfrequente Term mit Relaxationszeiten zwischen 5 ns und 18 ns, der in allen Spektren außer denen der Glukose-Lösungen auftritt, wird der teilweisen Rotation um die glykosidischen Bindungswinkel $\varphi$ und $\psi$ zugeschrieben. Als Beleg sind in Abbildung 66 die Spektren von Glukose-, Maltose- und $\mathrm{C}_{6} \mathrm{G}_{1}$-Lösungen bei jeweils $200 \mathrm{mmol} / \mathrm{L}$ dargestellt, wobei der entsprechende Absorptionsbeitrag für die beiden letzteren jeweils durch eine gestrichelte Linie hervorgehoben ist. Wie zu erwarten, weist das Glukose-Spektrum mangels glykosidischer Bindungen diesen Beitrag nicht auf, während die Beiträge im Spektrum der Maltose (glykosidische Bindung zwischen zwei Glukose-Einheiten) und im Spektrum von $\mathrm{C}_{6} \mathrm{G}_{1}$ (glykosidische Bindung zwischen einer GlukoseEinheit und einer Alkylkette) sowohl in Amplitude als auch in Relaxationszeit in etwa übereinstimmen.

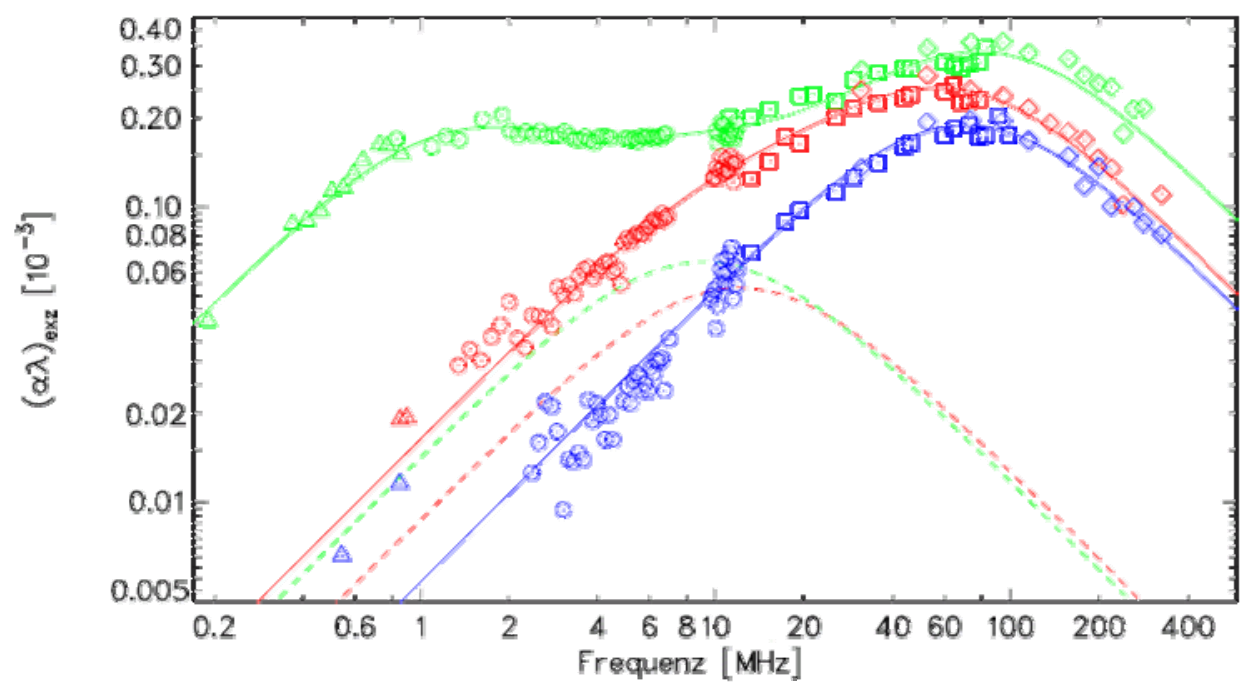

Abbildung 66: Ultraschallabsorptionsspektren von jeweils $200 \mathrm{mmol} / \mathrm{L}-\mathrm{Lösungen} \mathrm{von} \mathrm{Glukose}$ (blau), Maltose (rot) und $\mathbf{C}_{\mathbf{6}} \mathbf{G}_{\mathbf{1}}$ (grün). Die gestrichelten Linien beschreiben die der teilweisen $\varphi-/ \psi$ Rotation zuzuordnenden Debye-Spektralfunktionen.

Bei Betrachtung des Verlaufes der Relaxationszeiten der verschiedenen Substanzen und Konzentrationen fällt auf, dass die Daten eine vergleichsweise hohe Streuung aufweisen. Dafür lassen sich drei plausible Erklärungen finden: Zum einen liegt der Prozess im Frequenzbereich gerade zwischen den (in den meisten Fällen stärker ausgeprägten) Prozessen des Monomeraustausches und der $\omega$-Rotation. Dadurch ist der entsprechende DebyeSpektralterm in den $(\alpha \lambda)_{e x z}$-Spektren nicht als gesondertes lokales Maximum auszumachen und die Parameter weisen eine sehr hohe Unsicherheit auf. Weiterhin ist für diesen Prozess die genaue Ursache nicht bekannt, sondern es ist lediglich anzunehmen, dass der Prozess mit einer molekularen Konformationsänderung im Bereich der glykosidischen Bindung zusammenhängt. Die in [38] geäußerte Vermutung, dass der Prozess einem Übergang zwischen den in Molekulardynamik-Simulationen ([39]) auftretenden vier lokalen Energie-Minima im Bereich der ,syn'-Konformation zuzuordnen ist, würde bedeuten, dass es sich hier möglicherweise nicht um einen einzigen Prozess mit diskreter Relaxationszeit handelt, sondern um bis zu sechs verschiedene Übergänge mit ähnlichen Relaxationszeiten. In diesem Fall wäre eine Anpassung mit mehreren Debye-Spektralfunktionen oder mit einer empirischen Hill-Funktion, die auf einer Relaxationszeitenverteilung basiert, zwar physikalisch 
,richtiger', eine solche Anpassung würde aber zu viele unbekannte Parameter beinhalten. Es ist daher offensichtlich, dass die gewählte Anpassung mit einer Debye-Spektralfunktion sehr sensibel gegenüber möglichen leichten Veränderungen der Konformationskinetik der glykosidischen Bindung ist. Letztendlich erscheint es auch offensichtlich, dass speziell diese Kinetik sehr stark von folgenden Faktoren abhängt:

- Anzahl und Art der glykosidischen Bindungen: Bei Maltose (Glukose-Glukose) und den Alkylglukosiden (Glukose-Alkyl) liegt nur eine glykosidische Bindung vor, bei den Alkylmaltosiden hingegen zwei (Glukose-Glukose, Glukose-Alkyl).

- Aggregationszustand der Moleküle: Die glykosidische Konformationskinetik eines Moleküls im mizellaren Zustand ist gegenüber dem monomeren Zustand sicherlich stark beeinflusst. Da allerdings keine Aufspaltung des Prozesses beobachtet werden kann, ist jede der Relaxationszeiten ein Mittelwert über beide Zustände.

- Mizellform: Auch die Form der Mizellen hat sicherlich einen entscheidenden Einfluss auf die glykosidische Konformationskinetik, da das Volumen pro Kopfgruppe von der Krümmung der Mizelloberfläche abhängt (siehe Abbildung 65).

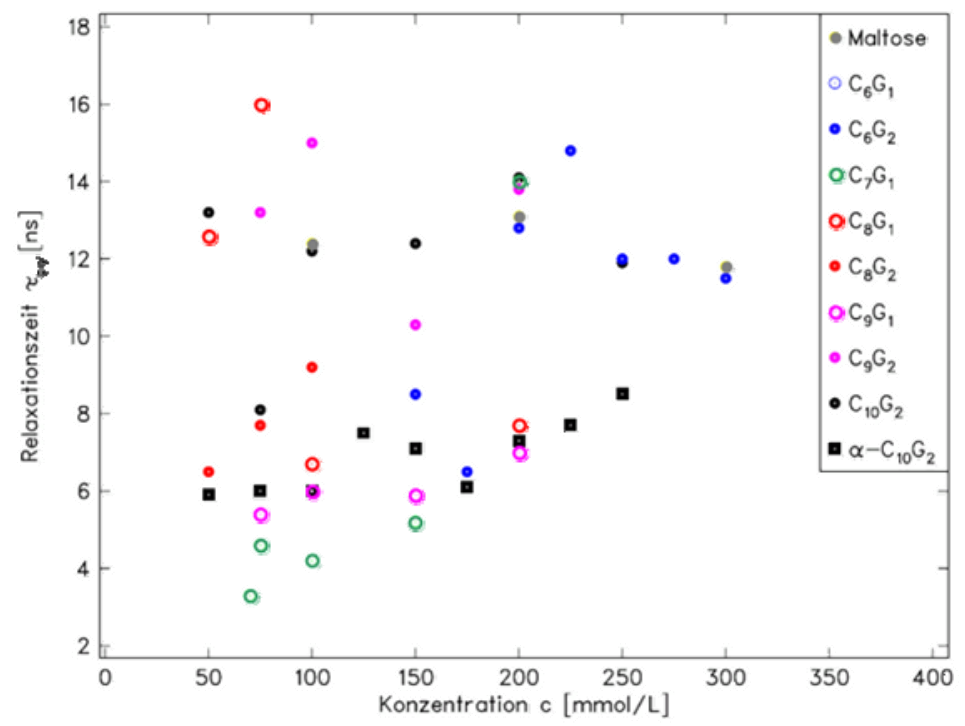

\section{Abbildung 67: Relaxationszeiten $\tau_{\varphi \psi}$ der (teilweisen) Rotation um die glykosidischen Bindungswin- kel $\varphi$ und $\psi$ als Funktion der Konzentration.}

Aus den oben genannten Gründen ist eine quantitative Analyse der Relaxationszeiten nicht möglich, es lassen sich jedoch in Abbildung 67 einige Trends erkennen, die im Folgenden qualitativ diskutiert werden:

- $\quad$ Bis auf wenige Ausnahmen liegen die Relaxationszeiten der Alkylglukoside im Bereich zwischen 4 ns und 8 ns nahezu unabhängig davon, ob sie als Monomere oder in Mizellen vorliegen. Dies deutet auf eine etwas schnellere Kinetik bei einer Bindung Glukose-Alkyl als bei Glukose-Glukose (Maltose: $\tau_{\varphi \psi} \approx 12 \mathrm{~ns}$ ) hin.

- Die Relaxationszeiten der Systeme $\mathrm{C}_{6} \mathrm{G}_{2}, \mathrm{C}_{9} \mathrm{G}_{2}$ und $\mathrm{C}_{10} \mathrm{G}_{2}$ liegen im gleichen Bereich wie die der Maltose dies könnte darauf hindeuten, dass bei den Alkylglykosiden die Bindung Glukose-Glukose einen größeren Beitrag zur Ultraschallabsorption liefert als die Bindung Glukose-Alkyl. 
- Eine Ausnahme zum vorigen Trend stellen die Relaxationszeiten der $\mathrm{C}_{8} \mathrm{G}_{2}$-Lösungen dar, da diese eher im Bereich der Alkylglukoside liegen - möglicherweise ist hier die Mizellform der entscheidende Faktor.

- Eine weitere Ausnahme ist das System $\alpha-\mathrm{C}_{10} \mathrm{G}_{2}$, bei dem die Alkylkette axial an das Glykosid gebunden ist, statt äquatorial wie bei den anderen Systemen. Dementsprechend liegen bei $\alpha-\mathrm{C}_{10} \mathrm{G}_{2}$-Mizellen die Kopfgruppen tangential zum Mizellkern vor und nicht wie bei den übrigen Systemen radial. Die deutlich kleineren Relaxationszeiten der $\alpha-\mathrm{C}_{10} \mathrm{G}_{2}$-Lösungen gegenüber denen des Diastereomers $\mathrm{C}_{10} \mathrm{G}_{2}$ lassen zwei Interpretationsmöglichkeiten zu: Entweder wird die Kinetik der Glukose-Glukose-Bindung durch das ,Aufliegen’ auf der Mizelloberfläche deutlich beschleunigt oder sie wird drastisch eingeschränkt, so dass die Relaxationszeiten in diesem Fall wieder eher die Kinetik der Glukose-Alkyl-Bindung reflektieren.

Aus den gleichen Gründen, die eine quantitative Analyse der Relaxationszeiten unmöglich machen, kann auch für die Amplituden nur eine qualitative Analyse erfolgen. In Abbildung 68 sind die Amplituden über der Konzentration dargestellt.

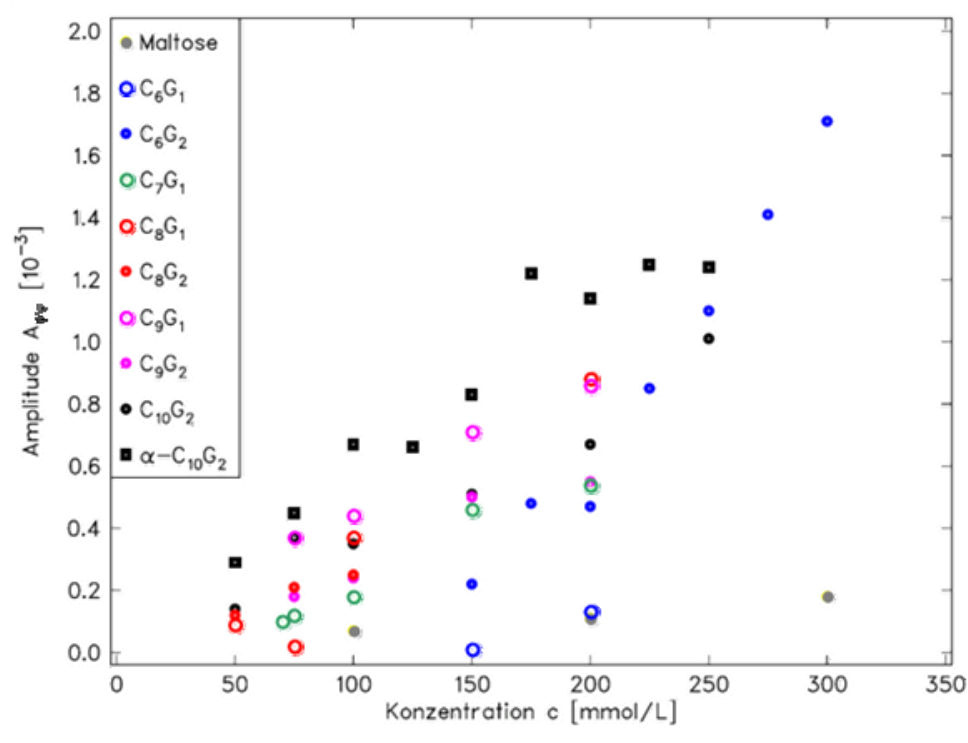

\section{Abbildung 68: Relaxationsamplituden $A_{\varphi \psi}$ des Terms, der der (teilweisen) Rotation um die glykosi- dischen Bindungswinkel $\varphi$ und $\psi$ zugeordnet wird als Funktion der Konzentration.}

Offensichtlich steigen die Amplituden, wie nach Gl. (2.19) und Gl. (2.24) zu erwarten, mit der Konzentration an, allerdings deutlich stärker als bei Maltose. Um diesen Effekt genauer zu untersuchen, ist in Abbildung 69 die skalierte Prozessamplitude $\tilde{A}_{\varphi \psi} / c$ gegen die Konzentration aufgetragen. Nach Gl. (2.19) ist $\tilde{A}_{\varphi \psi}=\Gamma \cdot\left(\Delta V_{S}\right)^{2}$ und nach Gl. (2.24) gilt für eine Konformationsänderung $\Gamma \sim c$. Dementsprechend sollte auch die Summe der reziproken Populationsanteile $\Sigma \equiv \Gamma / c$ (siehe Gl. (2.28)) konzentrationsunabhängig sein und damit auch die skalierte Prozessamplitude $\tilde{A}_{\varphi \psi} / c=\Sigma \cdot\left(\Delta V_{S}\right)^{2}$, da auch das Reaktionsvolumen $\Delta V_{S}$ nicht von der Konzentration abhängig ist. Es zeigt sich allerdings zum einen, dass die Werte für einige Systeme keineswegs konstant und zum anderen für fast alle Alkylglykoside signifikant höher sind als die Werte der Maltose-Lösungen (Mittelwert über alle Konzentrationen: $\left.\Sigma \cdot\left(\Delta V_{S}\right)^{2}=2 \cdot 10^{-7} \mathrm{~L}^{2} / \mathrm{mol}^{2}\right)$. Die Verläufe dieser Werte lassen sich im Einklang mit den aus den Verläufen der Relaxationszeiten resultierenden Vermutungen folgendermaßen qualitativ erklären: Bei dem System $\mathrm{C}_{6} \mathrm{G}_{1}(c m c \approx 250 \mathrm{mmol} / \mathrm{L})$ liegen die Moleküle zum Großteil noch als Monomere vor, so dass praktisch keine Beeinflussung des Prozesses durch, Mizellnachbarn' vorliegt. Die entsprechenden Werte $(c=150 \mathrm{mmol} / \mathrm{L}$ : 
$\left.\Sigma \cdot\left(\Delta V_{S}\right)^{2}=0.2 \cdot 10^{-7} \mathrm{~L}^{2} / \mathrm{mol}^{2} ; c=200 \mathrm{mmol} / \mathrm{L}: \Sigma \cdot\left(\Delta V_{S}\right)^{2}=0.7 \cdot 10^{-7} \mathrm{~L}^{2} / \mathrm{mol}^{2}\right)$ liegen deutlich unter denen der Maltose. Daraus folgt, dass bei einer Bindung Glukose-Alkyl gegenüber einer Bindung Glukose-Glukose entweder eine ungleichmäßigere Verteilung der möglichen Rotationszustände vorliegt (kleineres $\Sigma$, siehe Gl. (2.28)) oder ein kleineres Reaktionsvolumen $\Delta V_{S}$. Die Werte der Systeme $\mathrm{C}_{7} \mathrm{G}_{1}$ und $\mathrm{C}_{8} \mathrm{G}_{1}$ bestätigen diese Vermutung und weisen zudem genau wie die des Systems $\mathrm{C}_{9} \mathrm{G}_{1}$ daraufhin, dass das Produkt $\Sigma \cdot\left(\Delta V_{S}\right)^{2}$ für Moleküle in Mizellen bis zu 50-mal größer ist als für Monomere.

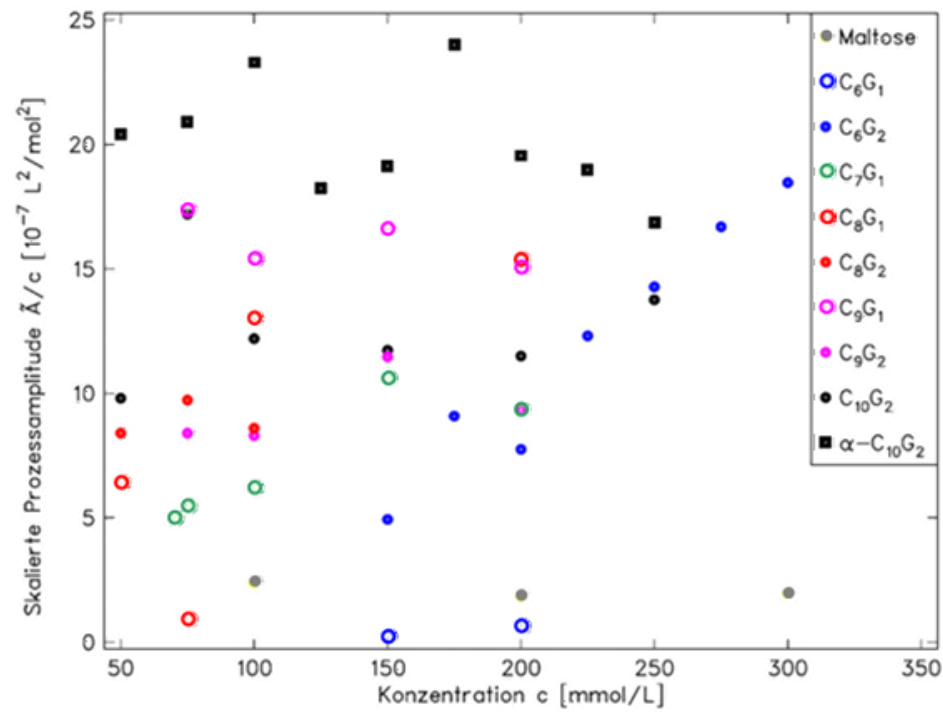

Abbildung 69: Skalierte Prozessamplitude $\tilde{A_{\varphi \psi}} / c$ des der (teilweisen) Rotation um die glykosidischen Bindungswinkel $\varphi$ und $\psi$ zugeordneten Relaxationsterms als Funktion der Konzentration.

Mit den obigen Erkenntnissen lassen sich für die ,reinen' Zustände folgende Werte grob abschätzen:

\begin{tabular}{r|c|c}
\hline \hline \multirow{2}{*}{ Bindungstyp } & monomer & mizellar \\
\hline \hline Glukose-Glukose & 2 & - \\
Glukose-Alkyl & 0.5 & 16 \\
\hline \hline
\end{tabular}

Tabelle 5.4: Abgeschätzte Werte für $\Sigma \cdot\left(\Delta V_{S}\right)^{2}$ in Abhängigkeit vom Typ der glykosidischen Bindung und vom Zustand des Moleküls. Alle Werte in $10^{-7} \mathrm{~L}^{2} / \mathrm{mol}^{2}$.

Die Verläufe der Werte der Alkylmaltosid-Lösungen erscheinen hingegen weniger einheitlich. Sie lassen sich daher nur durch ein Zusammenspiel mehrerer Faktoren erklären. So liegt bei den Systemen $\mathrm{C}_{8} \mathrm{G}_{2}, \mathrm{C}_{9} \mathrm{G}_{2}$ und $\mathrm{C}_{10} \mathrm{G}_{2}$ im Rahmen der Fehler ein konstanter Wert von etwa $10 \cdot 10^{-7} \mathrm{~L}^{2} / \mathrm{mol}^{2}$ vor. Dies bestätigt die bei der Analyse der Relaxationszeiten getroffene Vermutung, dass bei den Alkylmaltosiden die Bindung Glukose-Glukose einen größeren Einfluss auf die Ultraschallabsorptionsspektroskopie liefert als die Bindung Glukose-Alkyl, da für letztere ein größerer Wert zu erwarten wäre. Auch die etwa zweifach größeren Werte des Systems $\alpha-\mathrm{C}_{10} \mathrm{G}_{2}$ $\left(\Sigma \cdot\left(\Delta V_{S}\right)^{2} \approx 20 \cdot 10^{-7} \mathrm{~L}^{2} / \mathrm{mol}^{2}\right)$ gegenüber denen von $\mathrm{C}_{10} \mathrm{G}_{2}$ lassen sich in diesem Gesamtzusammenhang gut erklären - so folgt aus der oben getroffenen Vermutung, die vorliegenden Werte seien wegen der tangential auf der Mizelloberfläche aufliegenden $\alpha$-Kopfgruppen eher der Kinetik der Glukose-Alkyl-Bindung zuzuschreiben als jener der Glukose-Glukose-Bindung, dass hier der größere $\Sigma \cdot\left(\Delta V_{S}\right)^{2}$-Wert von $16 \cdot 10^{-7} \mathrm{~L}^{2} / \mathrm{mol}^{2}$ zu erwarten ist. 96 
Die Tatsache, dass die bestimmten Werte mit $\Sigma \cdot\left(\Delta V_{S}\right)^{2} \approx 20 \cdot 10^{-7} \mathrm{~L}^{2} / \mathrm{mol}^{2}$ noch größer sind, lässt wiederum vermuten, dass beim System $\alpha-\mathrm{C}_{10} \mathrm{G}_{2}$ wirklich beide verschiedenen Bindungstypen zu dem Gesamtwert beitragen. Schlussendlich lassen sich auch die Daten des Systems $\mathrm{C}_{6} \mathrm{G}_{2}$ plausibel erklären. Bei der niedrigsten untersuchten Konzentration $\left(c=150 \mathrm{mmol} / \mathrm{L}, \Sigma \cdot\left(\Delta V_{S}\right)^{2} \approx 5 \cdot 10^{-7} \mathrm{~L}^{2} / \mathrm{mol}^{2}\right)$ liegt der Großteil der Moleküle noch in monomerer Form vor und beide Bindungen können unbeeinflusst von Mizellnachbarn rotieren, so dass der entsprechende Wert in etwa der Summe aus den beiden Werten für den monomeren Zustand (Glukose-Glukose: $\Sigma \cdot\left(\Delta V_{S}\right)^{2} \approx 2 \cdot 10^{-7}$ $\mathrm{L}^{2} / \mathrm{mol}^{2}$; Glukose-Alkyl: $\Sigma \cdot\left(\Delta V_{S}\right)^{2} \approx 0.5 \cdot 10^{-7} \mathrm{~L}^{2} / \mathrm{mol}^{2}$, siehe Tabelle 5.4) entspricht. Bei Erhöhung der Konzentration liegt jedoch ein wachsender Anteil der Moleküle in Mizellen vor und der Wert $\Sigma \cdot\left(\Delta V_{S}\right)^{2}$ steigt auf etwa $20 \cdot 10^{-}$ ${ }^{7} \mathrm{~L}^{2} / \mathrm{mol}^{2}$ an. Dies deutet daraufhin, dass wie beim System $\alpha-\mathrm{C}_{10} \mathrm{G}_{2}$ beide Bindungstypen zu dem Gesamtwert beitragen und beide Bindungstypen durch die Mizellnachbarn beeinflusst werden. Im Gegensatz zu den Systemen $\mathrm{C}_{8} \mathrm{G}_{2}, \mathrm{C}_{9} \mathrm{G}_{2}$ und $\mathrm{C}_{10} \mathrm{G}_{2}$ wird allerdings die Glukose-Alkyl-Bindung nicht so stark beeinflusst, dass sie nahezu überhaupt nicht mehr zum Gesamtwert beiträgt. Erklären lässt sich dies plausibel mit der unterschiedlichen Mizellstruktur - während bei den Systemen $\mathrm{C}_{8} \mathrm{G}_{2}$ (mittlere Aggregationszahl: $\left.\bar{m} \approx 47\right), \mathrm{C}_{9} \mathrm{G}_{2}(\bar{m} \approx 55)$ und $\mathrm{C}_{10} \mathrm{G}_{2}$ ( $\bar{m} \approx 69)$ relativ große Aggregate vorliegen, in denen die Moleküle vergleichsweise dicht gepackt sind, ist die Struktur der $\mathrm{C}_{6} \mathrm{G}_{2}$-Mizellen $(\bar{m} \approx 30)$ deutlich offener.

Leider lässt sich nicht klären, ob die Änderungen der $\Sigma \cdot\left(\Delta V_{S}\right)^{2}$-Werte durch eine Änderung des Populationsfaktors $\Sigma$, des Reaktionsvolumens $\Delta V_{S}$ oder beider Größen verursacht werden, solange keine der beiden Größen sicher bekannt ist. Um dennoch einen groben quantitativen Eindruck zu gewinnen, ist in der folgenden Tabelle das Reaktionsvolumen $\Delta V_{S}$ für die verschiedenen Bindungstypen und Molekülzustände berechnet, jeweils unter den Annahmen, dass zwei, drei oder vier mögliche gleichgesetzte Rotationszustände vorliegen und dass die $\mathrm{Zu}-$ standspopulationen weder vom Bindungstyp abhängen noch davon, ob das Molekül als Monomer oder in einer Mizelle vorliegt. Die Werte sind allerdings nur als sehr grobe Richtwerte anzusehen, da es sehr wahrscheinlich ist, dass sich auch die Population der möglichen Rotationszustände in den einzelnen Fällen stark unterscheidet. Weiterhin liegt diesen Werten die Annahme zugrunde, dass sowohl das Reaktionsvolumen als auch die Relaxationszeit für alle möglichen Übergänge zwischen den einzelnen Rotationszuständen gleich sind.

\begin{tabular}{|c|c|c|c|c|}
\hline \multirow[b]{2}{*}{ Bindung } & \multirow[b]{2}{*}{ Zustand } & \multicolumn{3}{|c|}{ Zahl der Rotationszustände } \\
\hline & & 2 & 3 & 4 \\
\hline \multirow{2}{*}{ Glukose-Glukose } & monomer & 0.9 & 1.3 & 1.8 \\
\hline & mizellar & 2 & 3 & 4 \\
\hline \multirow{2}{*}{ Glukose-Alkyl } & monomer & 0.4 & 0.7 & 0.9 \\
\hline & mizellar & 2.5 & 4 & 5 \\
\hline
\end{tabular}

Tabelle 5.5: Abschätzung des Reaktionsvolumens der glykosidischen Rotation für verschiedene Bindungstypen sowie Zustände des Moleküls und mögliche Rotationszustände. Alle Werte in $\mathrm{mL} / \mathrm{mol}$. Erläuterungen zu den zugrundeliegenden Annahmen im Text. 


\subsection{Die Rotation der exozyklischen Gruppe ( $\omega$-Rotation)}

Als Beleg dafür, dass der hochfrequente Relaxationsterm der Rotation der exozyklischen $\mathrm{CH}_{2} \mathrm{OH}$-Gruppe zuzuordnen ist, sind analog zu Abbildung 56 und Abbildung 66 wieder die Ultraschallabsorptionsspektren von Lösungen jeweils der Konzentration $200 \mathrm{mmol} / \mathrm{L}$ Glukose, Maltose und $\mathrm{C}_{6} \mathrm{G}_{2}$ dargestellt, wobei diesmal der hochfrequente Absorptionsterm durch gestrichelte Linien gekennzeichnet ist. Es zeigt sich, dass der Prozess bei dem Alkylglykosid $\mathrm{C}_{6} \mathrm{G}_{2}$ in Hinsicht auf die Relaxationszeit und die Größenordnung der Amplitude sehr gut jenem bei Glukose und Maltose entspricht, der in [45] - [47] der $\omega$-Rotation zugeordnet werden konnte.

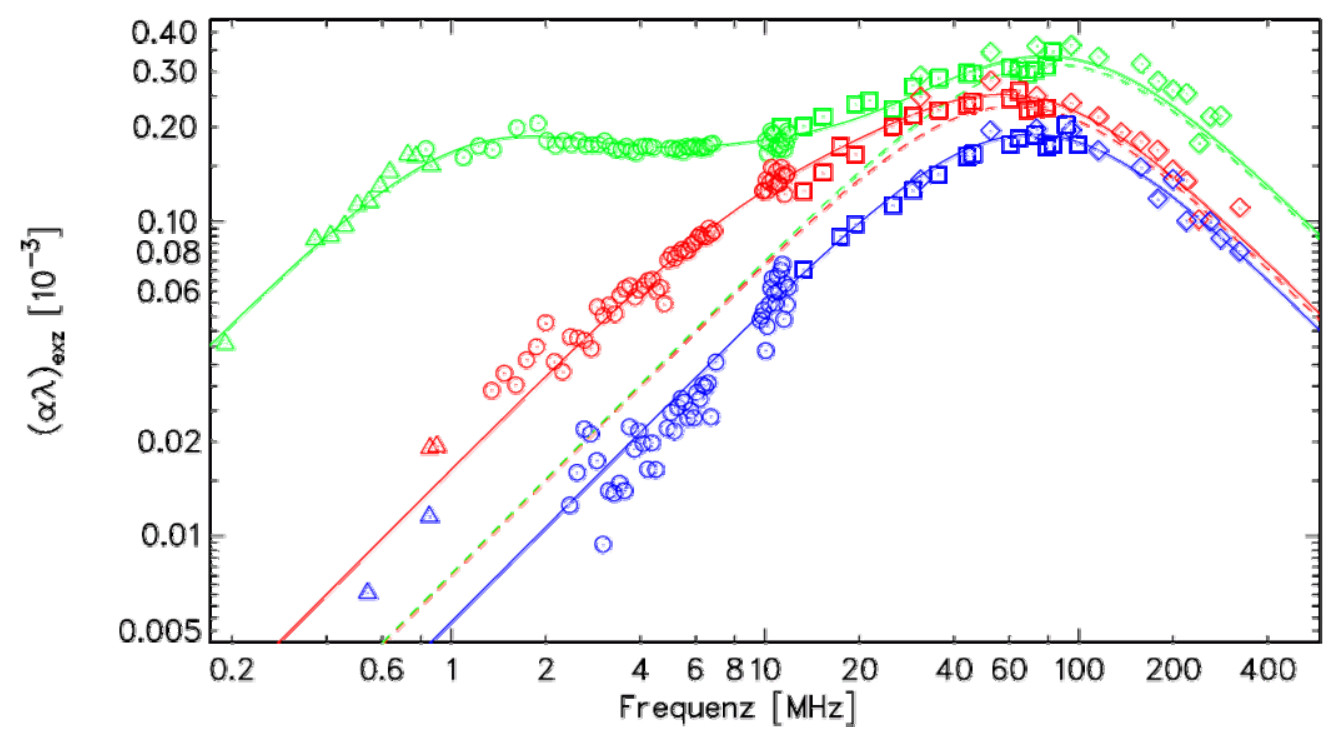

Abbildung 70: Ultraschallabsorptionsspektren für Lösungen von jeweils $200 \mathrm{mmol} / \mathrm{L}$ Glukose (blau), Maltose (rot) und $\mathbf{C}_{\mathbf{6}} \mathbf{G}_{\mathbf{1}}$ (grün). Die gestrichelten Linien beschreiben den der $\omega$-Rotation zuzuordnenden Debye-Spektralterm. Bei der Glukose entspricht dies der gesamten Zusatzabsorptionsfunktion in dem betrachteten Frequenzbereich (durchgezogene Linie).

In Abbildung 71 sind die Relaxationszeiten der $\omega$-Rotation über der Konzentration aufgetragen. Es fällt auf, dass alle Verläufe im Rahmen der Fehler konstant über der Konzentration sind und sich fast alle im Bereich $2 \pm 1 \mathrm{~ns}$ befinden. Die Grafik zeigt, dass die Relaxationszeiten (wie schon die der Rotation um die glykosidische Bindung) offenbar bevorzugt in zwei Bereichen vorliegen, hier $\approx 1.2$ ns und $\approx 2.0$ ns. Es kann nicht ausgeschlossen werden, dass auch hier durch die Anwesenheit von ,Mizellnachbarn’ die Kinetik des Prozesses beschleunigt oder verlangsamt wird. Da die Relaxationszeit dieses Prozesses mit einem vergleichsweise großen Fehler behaftet ist und in einigen Fällen bei der Anpassrechnung festgehalten werden musste, wird auf eine qualitative Analyse, wie sie bei der Rotation der glykosidischen Bindung vorgenommen wurde, verzichtet. Stattdessen soll aus den Relaxationszeiten eine Abschätzung der Reaktionskonstanten vorgenommen werden: Nach Gl. (2.25) ist $\tau_{\omega}=\left(k_{f}+k_{b}\right)^{-1}$ und nach Gl. (2.11) ist das Verhältnis der Reaktionskonstanten gleich dem der Rotamerpopulationen: $k_{f} / k_{b}=[\mathrm{gt}] /[\mathrm{gg}]$ (unter Vernachlässigung der geringen [tg]-Population). Für eine Abschätzung kann für Glukose angenommen werden, dass $[\mathrm{gt}] \approx[\mathrm{gg}]$ ist $([\mathrm{gt}]=45 \%,[\mathrm{gg}]=53 \%$, siehe Abbildung 18$)$. Mit $\tau_{\omega}=2 \mathrm{~ns}$ ergibt sich dann $k_{f} \approx k_{b} \approx 2.5 \cdot 10^{-8} \mathrm{~s}^{-1}$. 


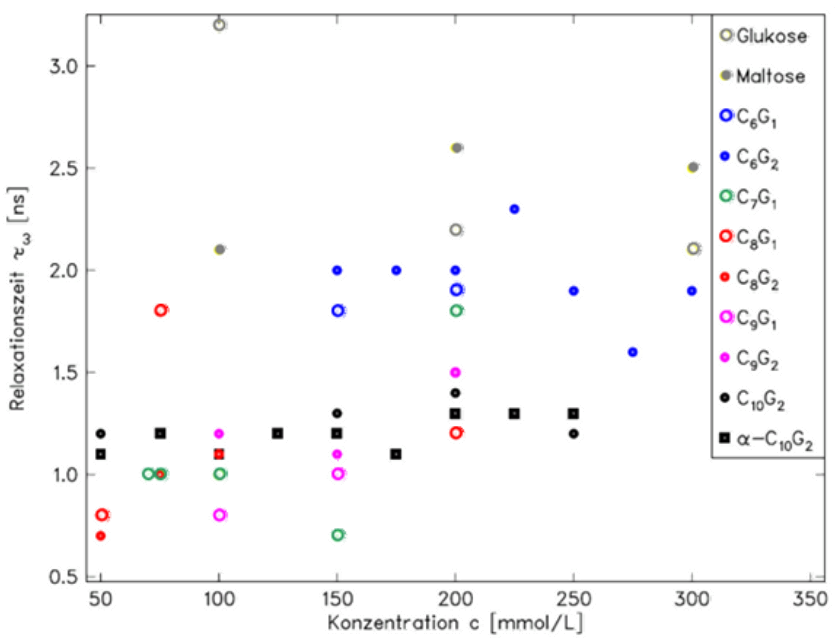

\section{Abbildung 71: Relaxationszeiten $\tau_{\omega}$ des $\omega$-Rotationsterms über der Konzentration.}

Eine tiefergehende qualitative und quantitative Analyse ermöglicht hingegen der in Abbildung 72 dargestellte Verlauf der Amplituden über der Konzentration. So kann zunächst mit den aus NMR-Messungen und MDSimulationen bekannten Rotamerpopulationen der Glukose (siehe Abbildung 18) nach den Gl. (2.19) und (2.24) aus den Amplituden das Reaktionsvolumen berechnet werden. Die Mittelung über die Werte der beiden in dieser Arbeit untersuchten Lösungen ergibt dabei ebenso einen Wert von $\Delta V_{S}=1.6 \mathrm{~mL} / \mathrm{mol}$ wie die Mittelung über die bereits vorliegenden Messungen aus [38]. Die Auswertung aus den Messungen an Maltose-Lösungen ist etwas komplexer, da das Maltosemolekül zwei exozyklischen Gruppen besitzt, deren Reaktionsvolumen zwar vermutlich gleich ist, deren Rotamerpopulationen sich nach [36] allerdings unterscheiden. Dementsprechend wird der Prozess als Überlagerung von zwei einzelnen Prozessen betrachtet und es gilt $\tilde{A}_{\omega}=\Gamma_{1} \cdot\left(\Delta V_{S}\right)^{2}+\Gamma_{2} \cdot\left(\Delta V_{S}\right)^{2}$ für die Prozessamplitude bzw. $\tilde{A}_{\omega} / c=\Sigma_{1} \cdot\left(\Delta V_{S}\right)^{2}+\Sigma_{2} \cdot\left(\Delta V_{S}\right)^{2}$ für die in Abbildung 73 aufgetragene skalierte Prozessamplitude $(\Sigma \equiv \Gamma / c$, siehe Gl. (2.28)). Mit dieser Berechnung ergibt sich für die drei in dieser Arbeit untersuchten Konzentrationen ein Mittelwert von $\Delta V_{S}=1.4 \mathrm{~mL} / \mathrm{mol}$, also ein etwas kleinerer Wert als für Glukose.

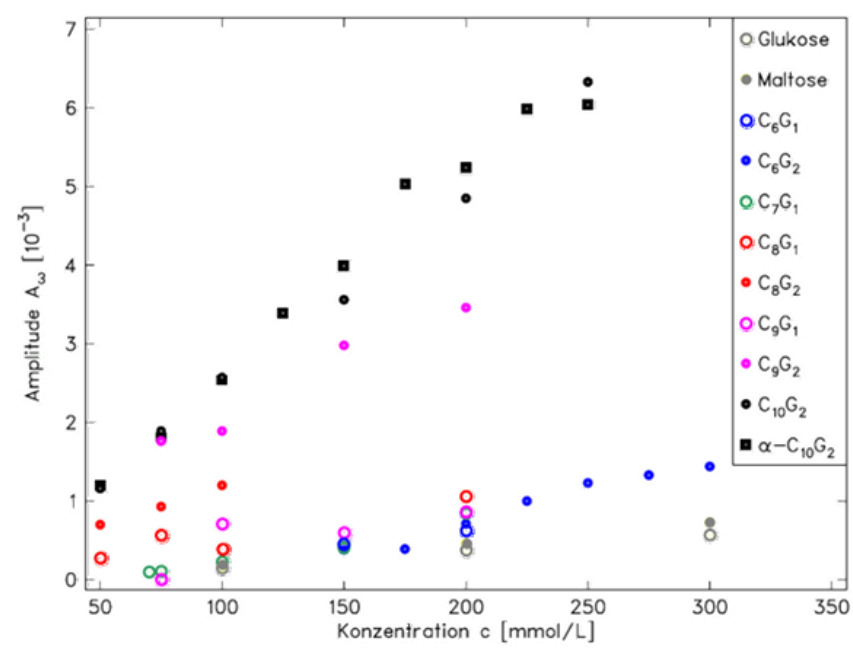

Abbildung 72: Amplituden $A_{\omega}$ des $\omega$-Rotationsterms über der Konzentration. 


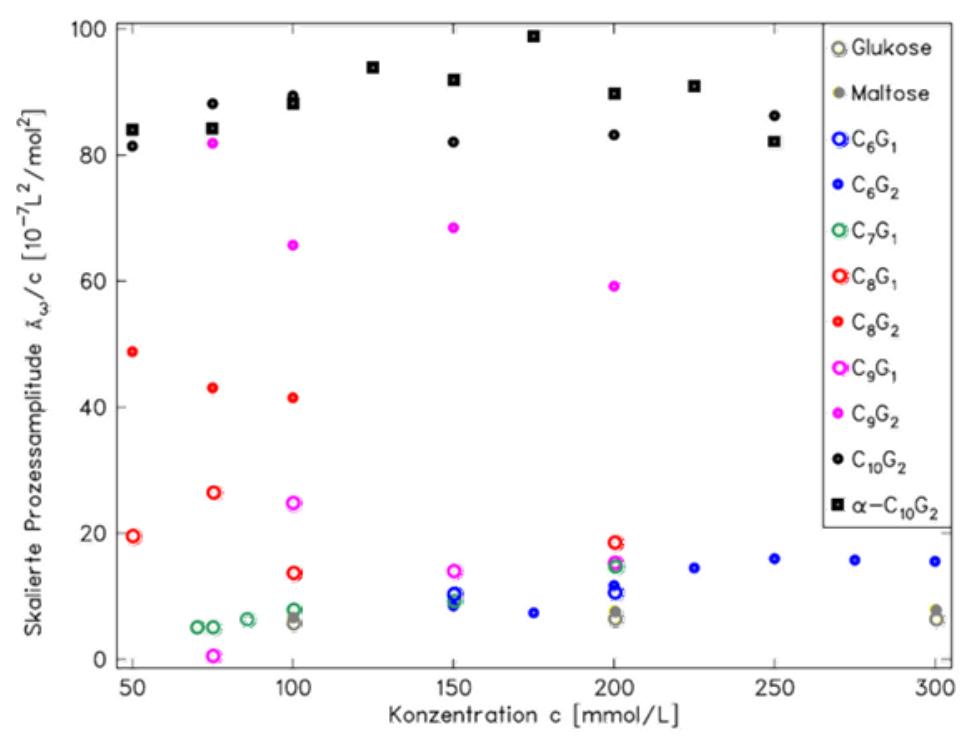

Abbildung 73: Skalierte Prozess-Amplituden $\tilde{A_{\omega}} / c$ des $\omega$-Rotationsterms über der Konzentration.

Die Verläufe der skalierten Prozess-Amplituden der Alkylglykosid-Lösungen über der Konzentration weisen deutlich darauf hin, dass die Größe $\tilde{A}_{\omega} / c=\Sigma \cdot\left(\Delta V_{S}\right)^{2}$ offenbar auch stark von der Kopfgruppe der Tenside und damit von der Anzahl der exozyklischen Gruppen sowie vom Zustand der Moleküle (monomer oder mizellar) abhängig ist. Eine exakte quantitative Analyse dieser Abhängigkeiten ist wiederum nicht möglich, da aus den Messdaten immer nur das Produkt $\Sigma \cdot\left(\Delta V_{S}\right)^{2}$ bestimmt werden kann und somit eine der beiden Größen definitiv bekannt sein müsste, um die andere bestimmen zu können. Allerdings kann für diesen Prozess eine Abschätzung des minimalen Reaktionsvolumens vorgenommen werden, da gegenüber der $\varphi$-/ $\psi$-Rotation einige Größen (insbesondere die Rotamerpopulationen bei Glukose und Maltose) bekannt sind. Berechnet man aus diesen Rotamerpopulationen die Größe $\Sigma$, erhält man Werte von $\Sigma=0.173$ (Maltose, nichtreduzierender Glukose-Ring) bis $\Sigma=0.243$ (Glukose). Da die Größe $\Sigma$ aber auf keinen Fall größer als 0.25 werden kann, ist offensichtlich, dass die teilweise deutlich größeren Werte der skalierten Prozessamplitude für die Alkylglykoside gegenüber Glukose und Maltose offenbar auf ein deutlich größeres Reaktionsvolumen $\Delta V_{S}$ zurückzuführen sind. Dementsprechend wird nun für alle Systeme $\Sigma^{\max }=0.25$ angenommen, um das minimale Reaktionsvolumen $\Delta V^{\min } \mathrm{zu}^{\text {berechnen }}{ }^{6}$. Weiterhin wird dieses minimale Reaktionsvolumen als Summe aus einzelnen Reaktionsvolumina betrachtet, die für die Zustände ,monomer' und ,mizellar' sowie im Falle der Alkylmaltoside für die beiden einzelnen GlukoseRinge als unterschiedlich angenommen werden müssen und die mit entsprechender Gewichtung zur Gesamtamplitude beitragen:

$$
\Sigma^{\max } \cdot c_{\text {ges }} \cdot\left(\Delta V^{\min }\right)^{2}=\Sigma^{\max } \cdot c_{\text {mon }} \cdot\left(\Delta V_{\text {mon }}^{\min }\right)^{2}+\Sigma^{\max } \cdot c_{m i z} \cdot\left(\Delta V_{m i z}^{\min }\right)^{2}
$$

für die Alkylglukoside bzw.

\footnotetext{
${ }^{6} \mathrm{Da}$ in dieser Arbeit immer das isentropische Reaktionsvolumen gemeint ist (siehe Gl. (2.22)), wird im Folgenden zur Reduzierung der Indizes der entsprechende Index „S“ weggelassen. 


$$
\begin{aligned}
\Sigma^{\max } \cdot c_{\text {ges }} \cdot\left(\Delta V^{\min }\right)^{2} & =\Sigma^{\max } \cdot c_{m o n, 1} \cdot\left(\Delta V_{\text {mon }, 1}^{\min }\right)^{2}+\Sigma^{\max } \cdot c_{\text {mon }, 2} \cdot\left(\Delta V_{\text {mon }, 2}^{\min }\right)^{2} \\
& +\Sigma^{\max } \cdot c_{m i z, 1} \cdot\left(\Delta V_{m i z, 1}^{\min }\right)^{2}+\Sigma^{\max } \cdot c_{m i z, 2} \cdot\left(\Delta V_{m i z, 2}^{\min }\right)^{2}
\end{aligned}
$$

für die Alkylmaltoside. Hierbei bezeichnen die Indizes ,mon' und ,miz' den Zustand des Moleküls und die Indizes 1 und 2 den ersten oder zweiten Glukose-Ring. Die Konzentration der Moleküle im monomeren Zustand $c_{m o n, 1}=c_{m o n, 2}=c_{m o n}$ kann dabei gemäß Gl. (3.1) und die der Moleküle im mizellaren Zustand $c_{m i z, l}=c_{m i z, 2}=c_{m i z}$ gemäß Gl. (3.2) berechnet werden.

Nun werden zunächst die Fälle betrachtet, in denen die Konzentration mizellarer Moleküle gleich Null oder zumindest vernachlässigbar klein ist, nämlich die $\mathrm{C}_{6} \mathrm{G}_{1}$ - und $\mathrm{C}_{6} \mathrm{G}_{2}$-Lösungen bei jeweils $150 \mathrm{mmol} / \mathrm{L}(c<c m c$ $\rightarrow c_{\text {miz }}=0$ ). Hier ergibt sich für $\mathrm{C}_{6} \mathrm{G}_{1}: \tilde{A} / c^{2}=\Sigma^{\max } .\left(\Delta V_{\text {mon }}^{\text {min }}\right)^{2}=1.02 \mathrm{~mL}^{2} / \mathrm{mol}^{2} \rightarrow \Delta V_{\text {mon }}^{\text {min }}=2.02 \mathrm{~mL} / \mathrm{mol}$ und für $\mathrm{C}_{6} \mathrm{G}_{2}$ : $\tilde{A} / c=2 \cdot \Sigma^{\max } \cdot\left(\Delta V_{\text {mon }}^{\text {min }}\right)^{2}=0.85 \mathrm{~mL}^{2} / \mathrm{mol}^{2} \rightarrow \Delta V_{\text {mon }}^{\text {min }}=1.3 \mathrm{~mL} / \mathrm{mol}$. Diese Werte sind im Rahmen der Fehler etwa so groß wie die von reiner Glukose bzw. Maltose. Dementsprechend wird im Folgenden $\Delta V_{\text {mon }}^{\min }=\Delta V_{G l u}=\Delta V_{M a l}=1.5$ $\mathrm{mL} / \mathrm{mol}$ angenommen. Nun kann für die Alkylglukoside mit Gl. (3.1) und Gl. (5.2) das Reaktionsvolumen im mizellaren Zustand abgeschätzt werden. Dabei ergeben sich für die drei Systeme $C_{7} G_{1}, C_{8} G_{1}$ und $C_{9} G_{1}$ Werte von etwa $\Delta V_{\text {miz }}=2.5 \mathrm{~mL} / \mathrm{mol}$ (siehe Abbildung 74). Nimmt man wiederum diesen Wert als eine Abschätzung für das minimale Reaktionsvolumen des äußeren Glukose-Rings der Alkylmaltoside, $\Delta V_{m i z, 1}^{\min }$, lässt sich daraus mit Gl. (3.2) und Gl. (5.3) das minimale Reaktionsvolumen des inneren Glukose-Rings, $\Delta V_{\operatorname{miz}, 2}^{\min }$, berechnen. Die entsprechenden Werte sind ebenfalls in Abbildung 74 gegen die Tensidkonzentration aufgetragen.

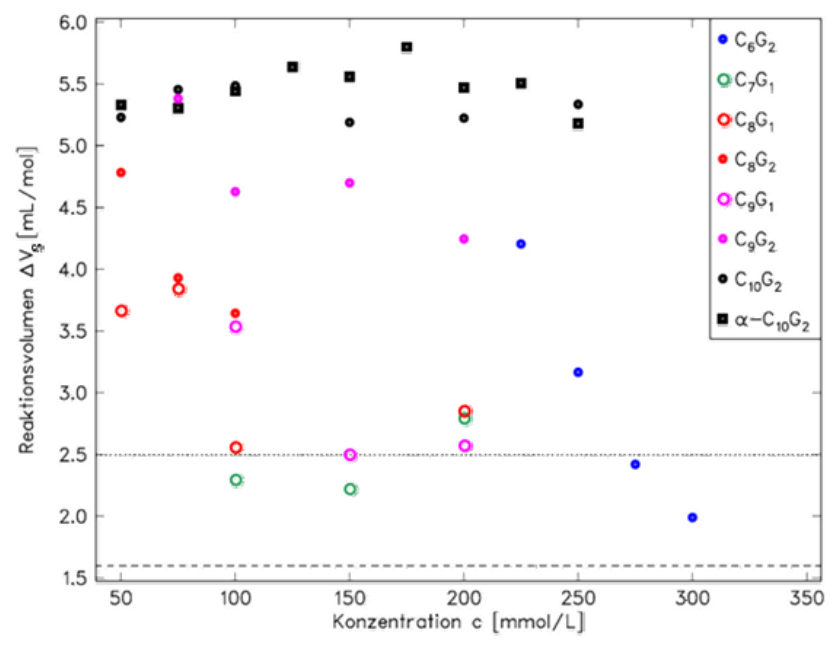

Abbildung 74: Minimales Reaktionsvolumen der Rotation der exozyklischen $\mathrm{CH}_{2} \mathrm{OH}$-Gruppe im mizellaren Zustand. Für die Alkylmaltoside ist das Reaktionsvolumen $\Delta V^{\min }{ }_{m i z, 2}$ des inneren GlukoseRings aufgetragen, berechnet mit dem Wert $\Delta V^{\text {min }}{ }_{\text {miz, }}=2.5 \mathrm{~mL} / \mathrm{mol}$ (gepunktete Linie) für den äußeren Glukose-Ring. Die gestrichelte Linie entspricht dem abgeschätzten Reaktionsvolumen im monomeren Zustand, $\Delta V^{\text {min }}{ }_{m o n}$, entsprechend dem von Glukose und Maltose. Erläuterungen zu den zugrunde liegenden Annahmen im Text.

Obwohl es sich bei den so berechneten Werten nur um grobe Abschätzungen handelt, lassen sich die Verläufe qualitativ plausibel mit den bisherigen Erkenntnissen in Einklang bringen: 
- Das Reaktionsvolumen bei den Alkylglukosiden ist im mizellaren Zustand mit $2.5 \mathrm{~mL} / \mathrm{mol}$ etwas größer als das im monomeren Zustand bzw. das von Glukose und Maltose mit etwa $1.5 \mathrm{~mL} / \mathrm{mol}$. Die Vergrößerung ist aufgrund der sterischen Bedingungen verständlich. Die Tatsache, dass sie nur vergleichsweise geringfügig ausfällt, ist zum einen dadurch zu erklären, dass es sich hier nur um die Abschätzung eines Minimalwertes handelt. Wenn die einzelnen Rotationszustände im mizellaren Zustand ungleichmäßiger besetzt sind als im monomeren Zustand, dann unterscheiden sich die Reaktionsvolumina auch stärker. Zum anderen zeigen hochgenaue Dichte- und Schallgeschwindigkeitsmessungen von $\mathrm{C}_{8} \mathrm{G}_{1}$-Lösungen, dass im hydrophilen Mizellmantel, also zwischen den Glukose-Köpfen, noch Wasser-Moleküle vorliegen [81]. Dementsprechend bedeutet eine Rotation der exozyklischen Gruppe im mizellaren Zustand nicht ausschließlich eine Interaktion mit benachbarten Tensidmolekülen, sondern zu einem gewissen Teil auch mit Wassermolekülen, so dass der Unterschied gegenüber dem monomeren Zustand nicht so groß ist.

- Die unter den erwähnten Annahmen durchgeführten Berechnungen der Reaktionsvolumina der inneren Glukose-Ringe der Alkylmaltoside, also jener, die sich näher am hydrophoben Mizellkern befinden, ergeben deutlich größere Werte von etwa $3.5 \mathrm{~mL} / \mathrm{mol}\left(\mathrm{C}_{8} \mathrm{G}_{2}\right)$ bis zu $6 \mathrm{~mL} / \mathrm{mol}\left(\mathrm{C}_{10} \mathrm{G}_{2}\right)$. Dies lässt sich darauf zurückführen, dass die Anzahl der Wassermoleküle bei Annäherung an den Mizellkern stark abnimmt und die exozyklischen Gruppen der inneren Glukose-Ringe dementsprechend nahezu ausschließlich mit anderen Tensidmolekülen interagieren. Eine derartige Interaktion ist offenbar mit einem deutlich größeren Reaktionsvolumen verbunden als eine Interaktion mit Wassermolekülen - anschaulich formuliert: die Nachbarmoleküle müssen für eine Rotation der exozyklischen $\mathrm{CH}_{2} \mathrm{OH}-\mathrm{Gruppe}$ ausweichen.

- Die Daten der Systeme $\mathrm{C}_{8} \mathrm{G}_{2}$ und $\mathrm{C}_{9} \mathrm{G}_{2}$ nehmen mit der Konzentration leicht $\left(\mathrm{C}_{9} \mathrm{G}_{2}\right)$ bzw. stark $\left(\mathrm{C}_{8} \mathrm{G}_{2}\right)$ ab bei dem System $\mathrm{C}_{8} \mathrm{G}_{2}$ wurde bereits eine mögliche Änderung der Mizellgeometrie mit der Konzentration als Ursache für die niedrigen Relaxationszeiten der Rotation der glykosidischen Bindung vermutet. Eine solche Geometrie-Änderung kann mit einem größeren Volumen pro Kopfgruppe einhergehen und somit mit einer weniger starken Interaktion mit den Mizellnachbarn, was die Abnahme der Daten plausibel erklären könnte.

- Der Verlauf der Daten des Systems $\mathrm{C}_{6} \mathrm{G}_{2}$ ist wiederum mit der niedrigen Aggregationszahl $(\bar{m} \approx 30) \mathrm{zu}$ begründen - die exozyklische Gruppe des inneren Glukose-Ringes interagiert wegen der losen Mizellstruktur eher noch mit Wassermolekülen als mit Mizellnachbarn und das Reaktionsvolumen ist daher deutlich geringer als bei den anderen Alkylmaltosiden. Die höheren Werte für das Reaktionsvolumen bei niedrigeren Konzentrationen sind dagegen vermutlich nicht physikalisch begründet, sondern beruhen auf der stark vereinfachten Berechnung der Konzentration mizellarer Moleküle gemäß Gl. (3.2).

- Erstaunlich ist die Tatsache, dass sich die berechneten Reaktionsvolumina der Systeme $\mathrm{C}_{10} \mathrm{G}_{2}$ und $\alpha-\mathrm{C}_{10} \mathrm{G}_{2}$ kaum unterscheiden. Da die Maltosid-Köpfe bei $\mathrm{C}_{10} \mathrm{G}_{2}$ radial in der Mizelloberfläche angeordnet sind, jene von $\alpha-\mathrm{C}_{10} \mathrm{G}_{2}$ hingegen tangential, ist zwischen beiden eigentlich ein deutlicher Unterschied zu erwarten. Eine plausible Erklärung für dieses Ergebnis ist, dass die einzelnen Maltosid-Köpfe von $\alpha-\mathrm{C}_{10} \mathrm{G}_{2}$ auf der Mizelloberfläche teilweise übereinander liegen und sich daher gegenseitig beeinflussen. 

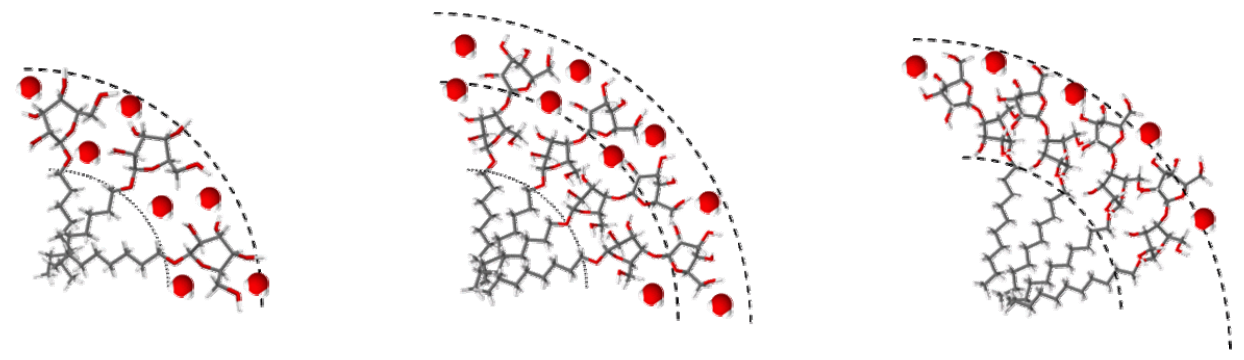

Abbildung 75: Schematische Darstellung eines Ausschnitts einer $C_{8} G_{1}$-Mizelle (links), einer $C_{8} G_{2^{-}}$ Mizelle (Mitte) und einer $\alpha-C_{10} G_{2}$-Mizelle (rechts) zur Frage der beeinflussenden Umgebung der einzelnen Kopfgruppen. Die $\mathrm{H}_{2} \mathrm{O}-$ Moleküle sind nur zur Verdeutlichung dargestellt. 


\subsection{Temperaturabhängigkeiten}

An dem System $\mathrm{C}_{8} \mathrm{G}_{1}$ /Wasser wurden bei drei Konzentrationen Messungen bei mehreren Temperaturen durchgeführt (siehe Tabelle 4.6). Aus der Literatur ist bekannt, dass die $c m c$ eine leichte Temperaturabhängigkeit aufweist (siehe Abbildung 76) und sich dementsprechend bei Variation der Temperatur einer Lösung das Verhältnis von Monomeren und Mizellen verändert. Hier soll daher anhand der Parameter der temperaturabhängigen Messungen diskutiert werden, ob die bisher getroffenen Aussagen über eine Beeinflussung der einzelnen Prozesse durch die Mizellnachbarn auch in diesem Fall bestätigt werden können.

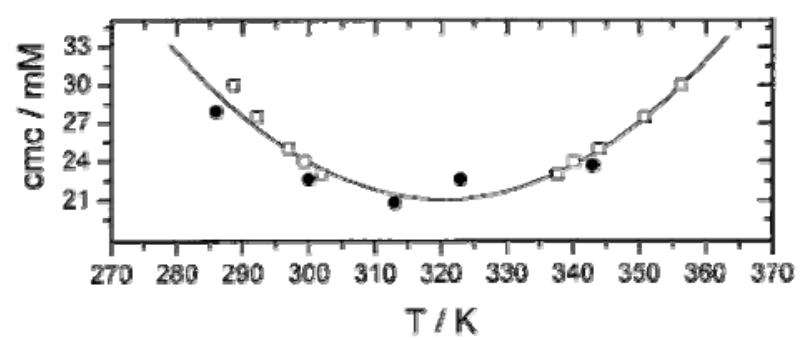

Abbildung 76: Temperaturabhängigkeit der $c m c$ von $C_{8} G_{1}$ aus Kalorimetrie-Messungen. Volle Punkte: Konzentrationsabhängige Messungen bei konstanter Temperatur. Offene Quadrate: Temperaturabhängige Messungen bei konstanter Konzentration. Grafik entnommen aus [115].

Auf eine genaue Auswertung der temperaturabhängigen Parameter des Monomeraustauschprozesses muss hier allerdings verzichtet werden, da zum einen nur bei zwei Konzentrationen entsprechend sichere Daten vorliegen und zum anderen für eine Auswertung die Verläufe $\bar{m}(T)$ und $\sigma(T)$ genau bekannt sein müssten, um sichere Aussagen zum Monomeraustausch treffen zu können.

Bei den Parametern der beiden Konformationsänderungen, also der Rotation der glykosidischen Bindung und der Rotation der exozyklischen Gruppe, fällt auf, dass die beiden Relaxationszeiten $\tau_{\varphi \psi}$ bzw. $\tau_{\omega}$ keine deutliche Abhängigkeit von der Temperatur aufweisen. Prinzipiell wäre hier eine Abnahme der Relaxationszeiten mit steigender Temperatur zu erwarten. Da allerdings die Relaxationszeitwerte eine relativ hohe Streuung aufweisen und zum Teil bei der Anpassrechnung festgehalten wurden und darüber hinaus der verfügbare Temperaturbereich klein ist, sollte diese fehlende Abnahme nicht überbewertet werden.

Wesentlich signifikanter und damit aussagekräftiger hingegen ist das Verhalten der Prozessamplituden $\tilde{A}_{\varphi \psi}$ und $\tilde{A_{\omega}}$ über der Temperatur. Während die Prozessamplitude $\tilde{A}_{\varphi \psi}$ der Rotation der glykosidischen Bindung mit steigender Temperatur deutlich abnimmt, weist jene der Rotation der exozyklischen Gruppe, $\tilde{A}_{\omega}$, ein genau umgekehrtes Verhalten auf (siehe Abbildung 77). Dieses zunächst ungewöhnliche Verhalten lässt sich durch Interaktion mit Mizellnachbarn erklären. Daher ist in Abbildung 78 für alle untersuchten Temperaturen die skalierte Prozessamplitude beider Prozesse gegen die reduzierte Konzentration aufgetragen. 


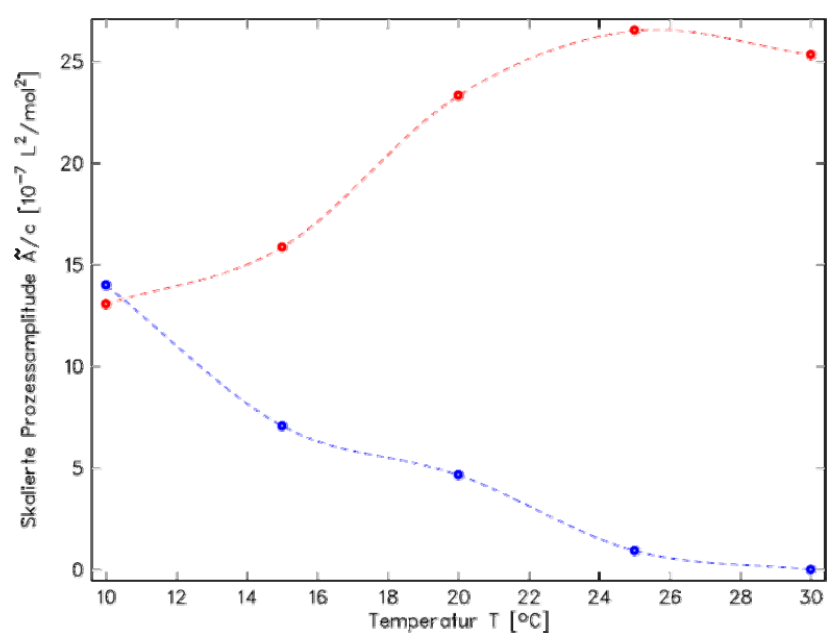

Abbildung 77: Temperaturabhängigkeit der skalierten Prozessamplitude des der $\varphi$-/ $\psi$-Rotation (blau) und des der $\omega$-Rotation (rot) zugeordneten Relaxationsterms. Die Linien dienen der Verdeutlichung des jeweiligen Verlaufes.

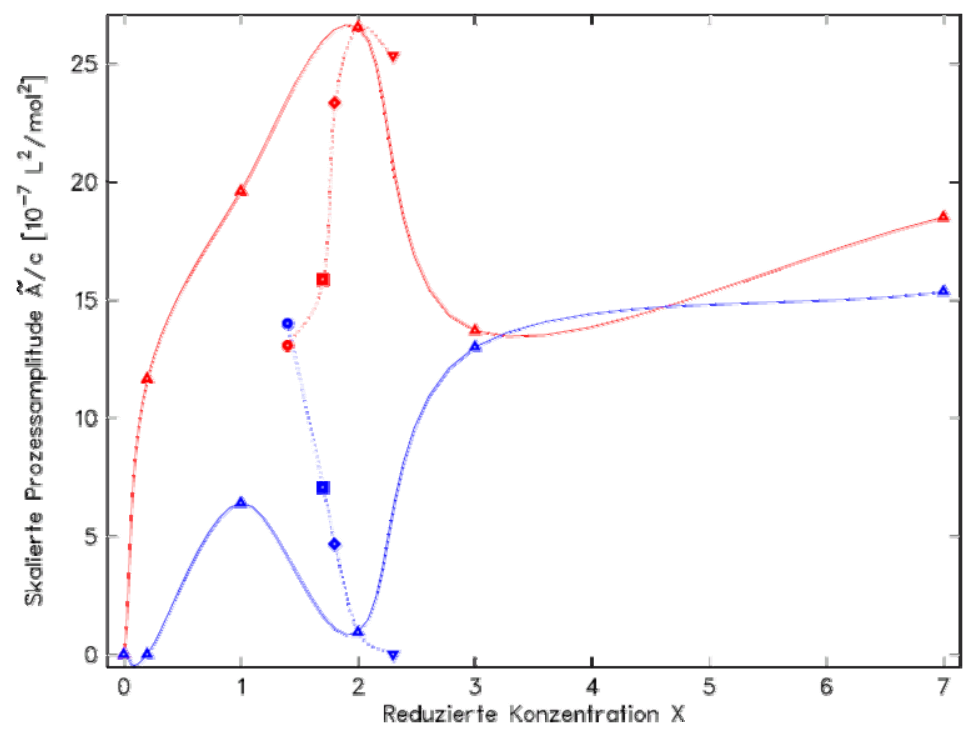

Abbildung 78: Abhängigkeit der skalierten Prozessamplitude des Terms für den $\varphi$-/ $\psi$-Prozess (blau) und den $\omega$-Prozess (rot) von der reduzierten Konzentration. Die durchgezogenen Linien ( $T=25$ $\left.{ }^{\circ} \mathrm{C}\right)$ und die gestrichelten Linien $(c=75 \mathrm{mmol} / \mathrm{L})$ dienen der Verdeutlichung der jeweiligen Verläufe. Die unterschiedlichen Symbole kennzeichnen unterschiedliche Temperaturen: Punkt: $10{ }^{\circ} \mathrm{C}$, Quadrat: $15{ }^{\circ} \mathrm{C}$, Raute: $20{ }^{\circ} \mathrm{C}$, Dreieck: $25{ }^{\circ} \mathrm{C}$, umgekehrtes Dreieck: $30{ }^{\circ} \mathrm{C}$.

Die obige Abbildung zeigt, dass der Verlauf der Parameter der temperaturabhängigen Messungen einen ähnlichen Trend aufweist wie jener der konzentrationsabhängigen Messungen. Beide Verläufe weisen ein Minimum von $\tilde{A}_{\varphi \psi}$ bzw. ein Maximum von $\tilde{A}_{\omega}$ bei einer reduzierten Konzentration von etwa $X \approx 2$ auf. Eine genaue Erklärung für diese Extrema kann nicht gegeben werden, aber in der Literatur finden sich Daten für das Achsenverhältnis elliptischer $\mathrm{C}_{8} \mathrm{G}_{1}$-Mizellen, die aus Dichte-, Viskositäts- und Dielektrizitäts-Messungen gewonnen wurden [116]. Diese Daten sind in Abbildung 79 gegen die reduzierte Konzentration aufgetragen. Die Daten sind zwar nur für reduzierte Konzentrationen größer als 4 angegeben, aber eine Extrapolation der Daten zu niedrigeren reduzierten Konzentrationen ergibt für $X=2$ ungefähr ein Achsenverhältnis von $J=1$, also kugelförmige Mizellen. Dementsprechend könnte bei $X=2$ eine Umwandlung von kugelförmigen Mizellen zu elliptischen oder 
zylindrischen Mizellen erfolgen. Dies wiederum könnte die Extrema in den beiden obigen Verläufen der skalierten Prozessamplituden erklären.

Ein zusätzlicher Hinweis, dass eine solche geometrische Umwandlung etwa bei $X=2$ stattfindet, findet sich bei der Auswertung der Parameter des Monomeraustauschprozesses (siehe Kapitel 5.1). Sowohl die Amplitude des Monomeraustauschprozesses (bzw. das Reaktionsvolumen pro $\mathrm{CH}_{2}$-Gruppe, siehe Abbildung 61) als auch das aus den Dichtedaten berechnete scheinbare molare Volumen (siehe Abbildung 62) entsprechen bis zu einer reduzierten Konzentration von etwa $X=2$ recht gut den Vorhersagen für kugelförmige Mizellen - bei höheren Konzentrationen sind jedoch sowohl das scheinbare molare Volumen als auch das Reaktionsvolumen kleiner als erwartet.

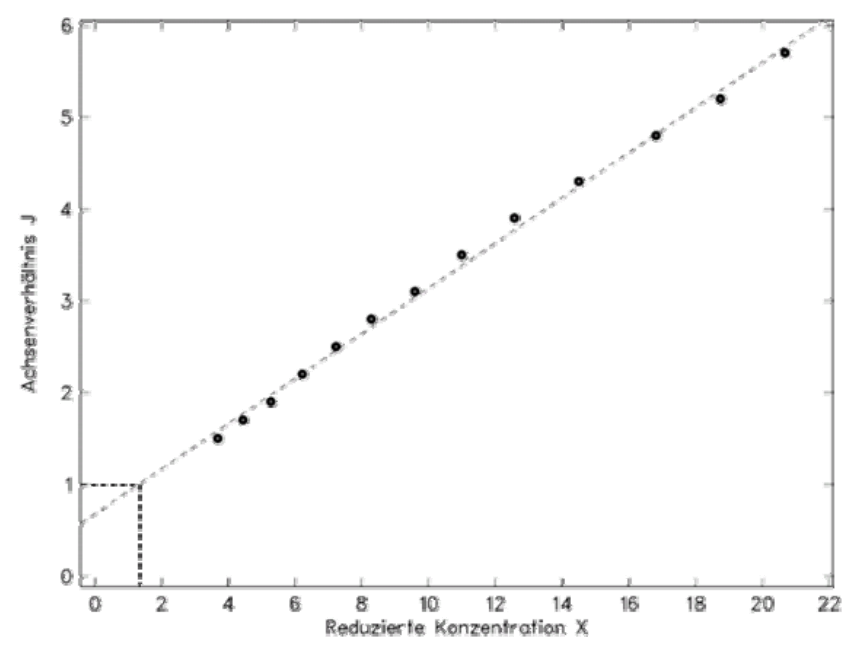

Abbildung 79: Achsenverhältnis $J$ von $C_{8} G_{1}$-Mizellen in Abhängigkeit von der reduzierten Konzentration $X$. Daten entnommen aus [116]. 


\title{
5.5 Einordnung der Ergebnisse für $\mathrm{C}_{8} \mathrm{G}_{1} / \mathrm{C}_{8} \mathrm{Gal}-\mathrm{Mischungen}$
}

Die in Abbildung 49 bzw. Abbildung 50 dargestellten Spektren für Lösungen von Mischungen aus $C_{8} G_{1}$ und $\mathrm{C}_{8} \mathrm{Gal}$ weisen auf den ersten Blick keine nennenswerten Unterschiede untereinander auf. Dies ist zunächst recht erstaunlich, da sich die Ultraschallabsorptionsspektren von Lösungen der beiden Kopfgruppen Glukose und Galaktose, wie in Abbildung 80 dargestellt, grundlegend voneinander unterscheiden. Allerdings zeigen die Parameter der einzelnen Relaxationsterme der $\mathrm{C}_{8} \mathrm{G}_{1} / \mathrm{C}_{8} \mathrm{Gal}$-Mischungen teilweise doch eine leichte Abhängigkeit von dem $\mathrm{C}_{8}$ Gal-Anteil. Diese Abhängigkeit der Parameter wird im Folgenden auf der Grundlage der bisherigen Erkenntnisse für die den einzelnen Relaxationstermen zugrunde liegenden Prozesse diskutiert.

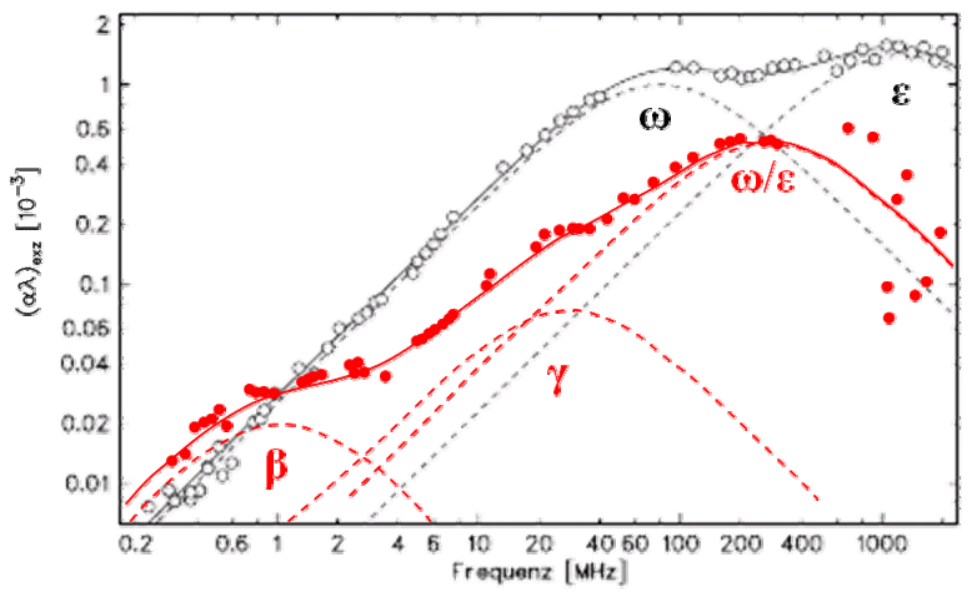

\begin{abstract}
Abbildung 80: Ultraschallabsorptionsspektren wässriger Lösungen von $1 \mathrm{~mol} / \mathrm{L}$ Glukose (schwarz) und $1 \mathrm{~mol} / \mathrm{L}$ Galaktose (rot). Die einzelnen Debye-Spektralfunktionen sind durch gestrichelte Linien gekennzeichnet. $\omega$ : Rotation der exozyklischen Gruppe, $\varepsilon$ : Assoziation, $\beta$ und $\gamma$ : teilweise Ringinversion (Pseudorotation). Daten stammen aus [34].
\end{abstract}

Zur Erklärung der beiden obigen Spektren: Während die Spektren der Glukose-Lösungen durchgehend zwei Relaxationsprozesse aufweisen, von denen einer der Rotation der exozyklischen Gruppe (" $\omega ")$ und einer einer Saccharid-Assoziation (" $\varepsilon ")$ zugeordnet werden können, treten in den Ultraschallabsorptionsspektren der Galaktose-Lösungen drei Relaxationsprozesse auf. Zwei davon (" $\beta$ " und " $\gamma$ ") werden einer teilweisen Ringinversion, der sogenannten Pseudorotation, zugeordnet, während für den dritten, hochfrequenten Term zwei unterschiedliche Interpretationen existieren. So wird in [34] für diesen Prozess die Rotation der exozyklischen Gruppe (" $\omega ")$ als Ursache vermutet, während in [38] die Zuordnung zu einem Assoziationsvorgang (" $\varepsilon ")$ bevorzugt wird. Beide Zuordnungen erscheinen in sich plausibel, insbesondere, da keine konzentrationsabhängigen Messungen vorliegen, aus denen eine klare Zuordnung zu einem intramolekularen Prozess (der $\omega$-Rotation) oder einem intermolekularen Prozess (der Assoziation) erfolgen könnte. Allerdings stellt sich bei beiden Interpretationen die Frage, warum der jeweils andere Prozess dann nicht in den Ultraschallabsorptionsspektren auftritt. 


\subsubsection{Der Monomeraustauschprozess}

In Abbildung 81 und Abbildung 82 sind die Relaxationsraten $\left(\tau_{\text {Mono }}\right)^{-1}$ bzw. die Amplituden $A_{\text {Mono }}$ des Monomeraustauschprozesses der $\mathrm{C}_{8} \mathrm{G}_{1} / \mathrm{C}_{8}$ Gal-Mischungen zusammen mit den Daten der reinen $\mathrm{C}_{8} \mathrm{G}_{1}$-Lösungen aufgetragen.

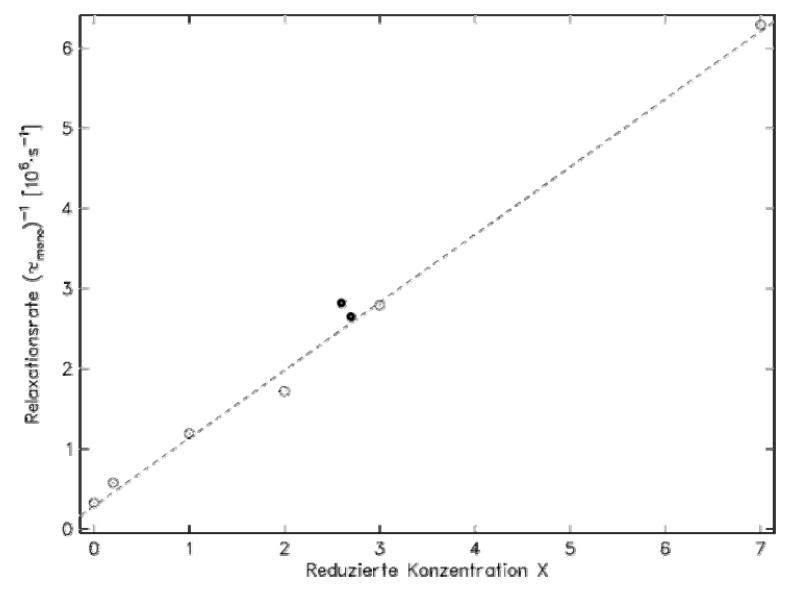

Abbildung 81: Relaxationsraten des Monomeraustauschprozesses der Lösungen beider $\mathrm{C}_{8} \mathrm{G}_{1} / \mathrm{C}_{8} \mathrm{Gal}$-Mischungen (Punkte) und der reinen $\mathrm{C}_{8} \mathrm{G}_{1}$-Lösungen (Kreise) als Funktion der reduzierten Konzentration.

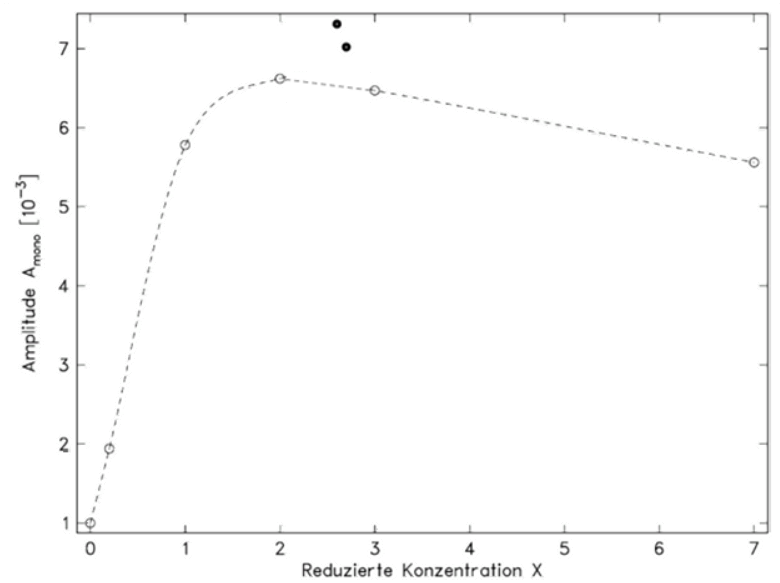

Abbildung 82: Amplituden des Monomeraustauschprozesses der beiden $\mathrm{C}_{8} \mathrm{G}_{1} / \mathrm{C}_{8} \mathrm{Gal}$-Lösungen (Punkte) und der reinen $\mathrm{C}_{8} \mathrm{G}_{1}$-Lösungen (Kreise) als Funktion der reduzierten Konzentration.

Die Relaxationsraten der $\mathrm{C}_{8} \mathrm{G}_{1} / \mathrm{C}_{8}$ Gal-Mischungen passen gut zu denen der reinen $\mathrm{C}_{8} \mathrm{G}_{1}$-Lösungen. Die Relaxationsamplituden sind im Falle der Mischungen etwas größer als aus dem Verlauf der $\mathrm{C}_{8} \mathrm{G}_{1}$-Lösungen folgend. Eine Berechnung der Reaktionsvolumina gemäß Gl. (3.7) ergibt hingegen $\Delta V=9.5 \mathrm{~mL} / \mathrm{mol}$ sowohl für die $\mathrm{C}_{8} \mathrm{G}_{1} / \mathrm{C}_{8} \mathrm{Gal}$-Mischungen als auch für die reinen $\mathrm{C}_{8} \mathrm{G}_{1}$-Lösungen. Daraus folgt, wie auch zu erwarten, dass der Monomeraustauschprozess durch die leichte Modifikation der Kopfgruppe kaum beeinflusst wird. 


\title{
5.5.2 Rotation der glykosidischen Bindung und der exozyklischen Grup- pe
}

Die Parameter der den beiden Konformationsänderungen zugeordneten Terme weisen leichte Abhängigkeiten vom $\mathrm{C}_{8} \mathrm{Gal}$-Anteil auf - während beide Relaxationszeiten durch die Zugabe von $\mathrm{C}_{8} \mathrm{Gal}$ etwas größer werden, nimmt die Prozessamplitude des $\varphi$-/ $\psi$-Rotationsterms ab, jene des $\omega$-Rotationsterms hingegen zu. Die skalierten Prozessamplituden sind in Abbildung 83 gegen die $\mathrm{C}_{8}$ Gal-Konzentration aufgetragen.

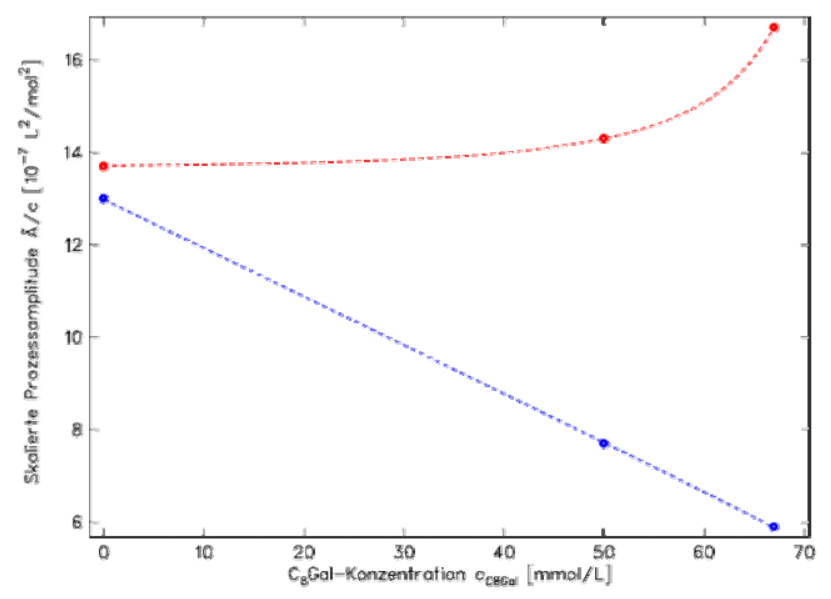

\begin{abstract}
Abbildung 83: Verlauf der skalierten Prozessamplituden der der $\varphi$-/ $\psi$-Rotation (blau) und der $\omega$ Rotation (rot) zugeordneten Terme für die $C_{8} G_{1} / C_{8} G a l-M i s c h u n g e n$ als Funktion der $C_{8}$ GalKonzentration. Die gestrichelten Linien dienen der Verdeutlichung des Verlaufs.
\end{abstract}

Für das Verhalten der Amplituden lassen sich zwei grundlegend verschiedene Erklärungsansätze finden. Zunächst liegt die Vermutung nahe, dass die $\mathrm{C}_{8}$ Gal-Moleküle mit einer größeren $(\omega)$ bzw. kleineren ( $\varphi \psi$ ) Prozessamplitude als die $\mathrm{C}_{8} \mathrm{G}_{1}$-Moleküle zur Gesamtamplitude beitragen. Im Falle der $\varphi$-/ $\psi$-Rotation würde dies bedeuten, dass $\Sigma_{\mathrm{C} 8 \mathrm{Gal}} \cdot\left(\Delta V_{\mathrm{C} 8 \mathrm{Gal}}\right)^{2}<\Sigma_{\mathrm{C} 8 \mathrm{G} 1} \cdot\left(\Delta V_{\mathrm{C} 8 \mathrm{G} 1}\right)^{2}$. Prinzipiell kann nicht ausgeschlossen werden, dass die möglichen Rotationszustände bei $\mathrm{C}_{8} \mathrm{Gal}$ ungleichmäßiger besetzt sind als bei $\mathrm{C}_{8} \mathrm{G}_{1}\left(\Sigma_{\mathrm{C} 8 \mathrm{Gal}}<\Sigma_{\mathrm{C} 8 \mathrm{G} 1}\right)$ oder dass das Reaktionsvolumen für $\mathrm{C}_{8} \mathrm{Gal}$ kleiner ist als für $\mathrm{C}_{8} \mathrm{G}_{1}$. Allerdings erscheint es sehr fraglich, ob die unterschiedliche Stellung der OH-Gruppe am $\mathrm{C}_{4}$-Atom bei den beiden Molekülen einen solchen Unterschied bei der Rotation der glykosidischen Bindung hervorrufen kann. Als noch weniger plausibel erweist sich die Annahme, dass die skalierte Prozessamplitude im Falle der $\omega$-Rotation bei den $\mathrm{C}_{8}$ Gal-Molekülen deutlich größer ist als die der $C_{8} G_{1}$ Moleküle. Wie aus Abbildung 18 ersichtlich, verursacht die axiale Stellung der $\mathrm{OH}$-Gruppe am $\mathrm{C}_{4}$-Atom der Galaktose eine wesentlich ungleichmäßigere Rotamerverteilung als die äquatoriale Stellung bei der Glukose und damit einen eher kleineren $\Sigma$-Wert (Galaktose: gt:gg=65:19 $\rightarrow \Sigma=\left(0.65^{-1}+0.19^{-1}\right)^{-1}=0.15$, Glukose: gt:gg=45:53 $\left.\rightarrow \Sigma=\left(0.45^{-1}+0.53^{-1}\right)^{-1}=0.24\right)$. Auch hier kann allerdings nicht mit völliger Sicherheit ausgeschlossen werden, dass die Anwesenheit der Mizellnachbarn bei den $\mathrm{C}_{8}$ Gal-Molekülen eine gleichmäßigere Rotamerpopulation oder ein größeres Reaktionsvolumen verursacht. Dennoch erscheint eine solche Begründung nicht sehr plausibel.

Eine deutlich einleuchtendere Erklärung für die Verläufe der beiden skalierten Prozessamplituden ergibt sich bei Betrachtung der Abhängigkeit von der reduzierten Konzentration statt vom Anteil der $\mathrm{C}_{8}$ Gal-Moleküle. Wie in 
Abbildung 84 sichtbar, fügen sich diese Abhängigkeiten sehr gut in den Verlauf der Daten für die $C_{8} G_{1}$ Lösungen ein.

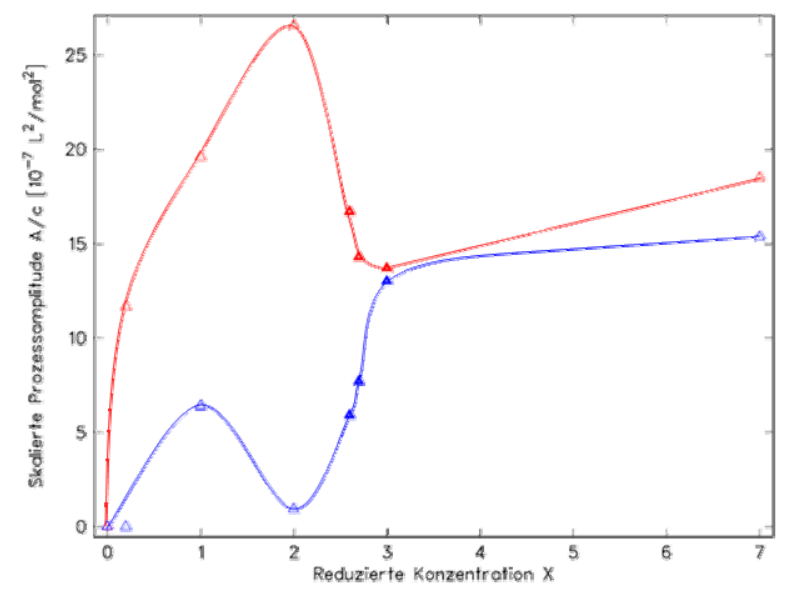

Abbildung 84: Abhängigkeit der skalierten Prozessamplitude der Terme für den $\varphi$-/ $\psi$-Prozess (blau) und den $\omega$-Prozess (rot) von der reduzierten Konzentration. Die offenen Symbole sind die Daten der reinen $\mathrm{C}_{8} \mathrm{G}_{1}$-Lösungen, die vollen Symbole die der $\mathrm{C}_{8} \mathrm{G} 1 / \mathrm{C}_{8} \mathrm{Gal}$-Mischungen. Die durchgezogenen Linien dienen der Verdeutlichung der jeweiligen Verläufe.

Möglicherweise sind also auch diese Verläufe eher durch die Änderung der reduzierten Konzentration der $\mathrm{C}_{8} \mathrm{G}_{1} / \mathrm{C}_{8} \mathrm{Gal}$-Mischungen als durch einen besonderen Effekt der $\mathrm{C}_{8} \mathrm{Gal}$-Moleküle zu erklären. Offenbar unterstützen daher auch die Messungen an den Lösungen von $\mathrm{C}_{8} \mathrm{G}_{1} / \mathrm{C}_{8}$ Gal-Mischungen die Vermutung, dass bei etwa $X=2$ eine drastische Änderung der Mizellgeometrie auftritt. Letztendlich sind aber für eine sichere Bestätigung dieser Vermutung und möglicherweise auch für eine quantitative Analyse dieses Effektes noch zahlreiche weitere Messungen vonnöten. 


\subsection{Komplexierung mit $\alpha$-Cyclodextrin}

Das Zusammenspiel zwischen der Mizellkinetik von Tensiden und der Komplexbildungsdynamik von Tensidund Cyclodextrin-Molekülen ist Gegenstand zahlreicher Arbeiten in der Literatur ([98]-[103], [117], [118]). Eine detaillierte Betrachtung der möglichen Komplexbildungs- und Aggregationsreaktionen zeigt, dass ein TensidMolekül in einer solchen Lösung entweder als freies Monomer oder in einem von vier verschiedenen Komplexen vorliegen kann (Mizelle, $\left.\mathrm{T}{ }^{\circ} \mathrm{CD}, \mathrm{T}_{2}{ }^{\circ} \mathrm{CD}, \mathrm{T}{ }^{\circ} \mathrm{CD}_{2}\right)^{7}$. Weiterhin kann jeder dieser Komplexe auf mehrere Arten gebildet werden, wie in Tabelle 5.6 schematisch dargestellt.

\begin{tabular}{|c|c|}
\hline Reaktion & Mögliche Komplexe \\
\hline $\mathrm{T}+\mathrm{T}_{i-1} \stackrel{K_{m}}{\rightleftharpoons} \mathrm{T}_{i}$ & $\begin{array}{l}\text { mizellare Aggregate der Größe } \\
i, i=2 \ldots N\end{array}$ \\
\hline $\mathrm{T}+\mathrm{CD} \stackrel{K_{11}}{\rightleftharpoons} \mathrm{T} \circ \mathrm{CD}$ & \\
\hline $\mathrm{T}+\mathrm{T} \circ \mathrm{CD} \stackrel{K_{21}}{\rightleftharpoons} \mathrm{T}_{2} \circ \mathrm{CD}$ & है। \\
\hline $\mathrm{T}+\mathrm{CD}_{2} \stackrel{K_{12}}{\rightleftharpoons} \mathrm{T} \circ \mathrm{CD}_{2}$ & 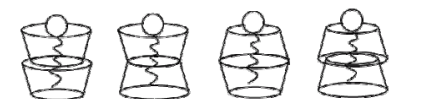 \\
\hline
\end{tabular}

Tabelle 5.6: Mögliche Komplexe, in denen ein Tensid-Molekül in einer Cyclodextrin/Tensid-Lösung vorliegen kann.

Da sowohl der Mizellbildungs-/Zerfallsprozess als auch die Bildung der einzelnen Komplexe nur mit freien Tensidmonomeren erfolgen kann, erhält man hier eigentlich ein gekoppeltes System aus allen vier Reaktionsgleichungen. Allerdings werden in der Literatur Hinweise gegeben, die eine getrennte Betrachtung des Monomeraustausches und der Komplexbildung rechtfertigen:

Zum einen können nach [20] Reaktionen getrennt betrachtet werden, wenn deren Relaxationszeiten sich um mehr als einen Faktor von etwa 10 unterscheiden, da in diesem Fall die schnellere Reaktion gegenüber der langsameren immer im Gleichgewicht ist. Da im vorliegenden Fall für alle Konzentrationen $\tau_{\text {mono }}>6 \cdot \tau_{\text {kompl }}$ gilt, werden hier die Reaktionen in guter Näherung als voneinander unabhängig betrachtet. Weiterhin wird in der Literatur allgemein davon ausgegangen, dass in Tensid-Cyclodextrin-Mischungen erst dann Mizellen gebildet werden, wenn die Konzentration freier, also nicht komplexierter Tensidmoleküle gleich der $c m c$ des Tensids ist. Die Konzentration, bei der das der Fall ist, wird als scheinbare $c m c^{*}$ bezeichnet. Oberhalb dieser scheinbaren $c m c^{*}$ bilden sich die Mizellen dann genau so aus wie in einer reinen Tensid-Lösung, also mit unveränderter mittlerer Aggregationszahl und unveränderten Reaktionskonstanten.

\footnotetext{
${ }^{7}$ Um die folgenden stöchiometrischen Berechnungen übersichtlicher zu gestalten, wird hier , $\mathrm{T}$ ' für Tensid anstatt $\mathrm{C}_{8} \mathrm{G}_{1}$ als Bezeichnung benutzt.
} 
Daher wird hier zunächst eine Betrachtung zur Stöchiometrie der Komplexbildung vorgenommen, um eine Abhängigkeit der Konzentration freier Tensidmonomere von der Gesamt-Tensidkonzentration zu erhalten. Dann kann überprüft werden, ob die Mizellkinetik in Abhängigkeit von dieser freien Monomer-Konzentration jener der reinen Tensid-Lösung entspricht.

Für die einzelnen in Tabelle 5.6 aufgeführten Reaktionen kann zunächst jeweils Gl. (2.13) angewandt werden:

$$
\begin{aligned}
& K_{11}=\frac{k_{f}^{11}}{k_{b}^{11}}=\frac{c(\mathrm{~T} \circ \mathrm{CD})}{c_{f r e i}(\mathrm{~T}) \cdot c_{f r e i}(\mathrm{CD})} \\
& K_{21}=\frac{k_{f}^{21}}{k_{b}^{21}}=\frac{c\left(\mathrm{~T}_{2} \circ \mathrm{CD}\right)}{c_{f r e i}(\mathrm{~T}) \cdot c(\mathrm{~T} \circ \mathrm{CD})} \\
& K_{12}=\frac{k_{f}^{12}}{k_{b}^{12}}=\frac{c\left(\mathrm{~T} \circ \mathrm{CD}_{2}\right)}{c(\mathrm{~T} \circ \mathrm{CD}) \cdot c_{f r e i}(\mathrm{CD})}
\end{aligned}
$$

Der Index ,frei“ wird hier für die Konzentration freier Moleküle benutzt, um Verwechslungen mit der Gesamtkonzentration zu vermeiden. ,Frei’ bedeutet hier zunächst lediglich ,nicht komplexiert' - eine Unterscheidung von mizellaren und monomeren Molekülen wird erst später vorgenommen. Weiterhin ist offensichtlich, dass sowohl das Tensid als auch das Cyclodextrin in einer der möglichen Formen vorliegen muss. Daraus folgt:

$$
\begin{aligned}
& c_{\text {ges }}(\mathrm{T})=c_{\text {frei }}(\mathrm{T})+c(\mathrm{~T} \circ \mathrm{CD})+2 \cdot c\left(\mathrm{~T}_{2} \circ \mathrm{CD}\right)+c\left(\mathrm{~T} \circ \mathrm{CD}_{2}\right) \\
& c_{\text {ges }}(\mathrm{CD})=c_{\text {frei }}(\mathrm{CD})+c(\mathrm{~T} \circ \mathrm{CD})+c\left(\mathrm{~T}_{2} \circ \mathrm{CD}\right)+2 \cdot c\left(\mathrm{~T} \circ \mathrm{CD}_{2}\right)
\end{aligned}
$$

In diesen Gleichungen können mithilfe von Gl. (5.4) bis (5.6) die Konzentrationen der Komplexe eliminiert werden:

$$
\begin{aligned}
c_{\text {ges }}(\mathrm{T})= & c_{\text {frei }}(\mathrm{T})+K_{11} \cdot c_{\text {frei }}(\mathrm{T}) \cdot c_{\text {frei }}(\mathrm{CD}) \\
& +2 \cdot K_{21} \cdot K_{11} \cdot c_{\text {frei }}{ }^{2}(\mathrm{~T}) \cdot c_{\text {frei }}(\mathrm{CD})+K_{12} \cdot K_{11} \cdot c_{\text {frei }}(\mathrm{T}) \cdot c_{\text {frei }}{ }^{2}(C D) \\
c_{\text {ges }}(\mathrm{CD}) & =c_{\text {frei }}(\mathrm{CD})+K_{11} \cdot c_{\text {frei }}(\mathrm{T}) \cdot c_{\text {frei }}(\mathrm{CD}) \\
& +K_{21} \cdot K_{11} \cdot c_{\text {frei }}{ }^{2}(\mathrm{~T}) \cdot c_{\text {frei }}(\mathrm{CD})+2 K_{12} K_{11} \cdot c_{\text {frei }}(\mathrm{T}) \cdot c_{\text {frei }}{ }^{2}(C D)
\end{aligned}
$$

Mit diesen Gleichungen ist nun bereits der gesuchte Zusammenhang zwischen der Konzentration freier Tensidmoleküle und der Gesamt-Tensidkonzentration hergestellt. Allerdings wäre eine Anpassung der Gleichungen an die vorliegenden Messdaten ohne weitere Daten noch nicht möglich. Es liegen jedoch Werte für die einzelnen Gleichgewichtskonstanten aus der Literatur vor $\left(K_{11}=1700 \mathrm{~L} / \mathrm{mol}, K_{21}=0 \mathrm{~L} / \mathrm{mol}, K_{12}=64 \mathrm{~L} / \mathrm{mol}\right.$ [117]), mit denen die einzelnen Konzentrationen berechnet werden können. Dass eine der Gleichgewichtskonstanten gleich Null ist, bedeutet, dass Komplexe der Form $\mathrm{T}_{2}{ }^{\circ} \mathrm{CD}$ sehr instabil sind und folglich kaum vorkommen. Generell ist an dieser Stelle darauf hinzuweisen, dass Gleichgewichtskonstanten eine sehr ungenau zu bestimmende Größe darstellen - für die Komplexbildung von $\beta$-Cyclodextrin mit dem kationischen Tensid Natriumdodecylsulfat existie- 
ren beispielsweise Literaturwerte von $K_{11}=210 \mathrm{~L} / \mathrm{mol}$ bis $K_{11}=25600 \mathrm{~L} / \mathrm{mol}$ ([118]). In Abbildung 85 sind die Verläufe der Konzentrationen der einzelnen Spezies, die aus Gl. (5.4) bis (5.10) berechnet wurden, gegen die Tensidkonzentration $c_{\text {ges }}(\mathrm{T})$ aufgetragen.

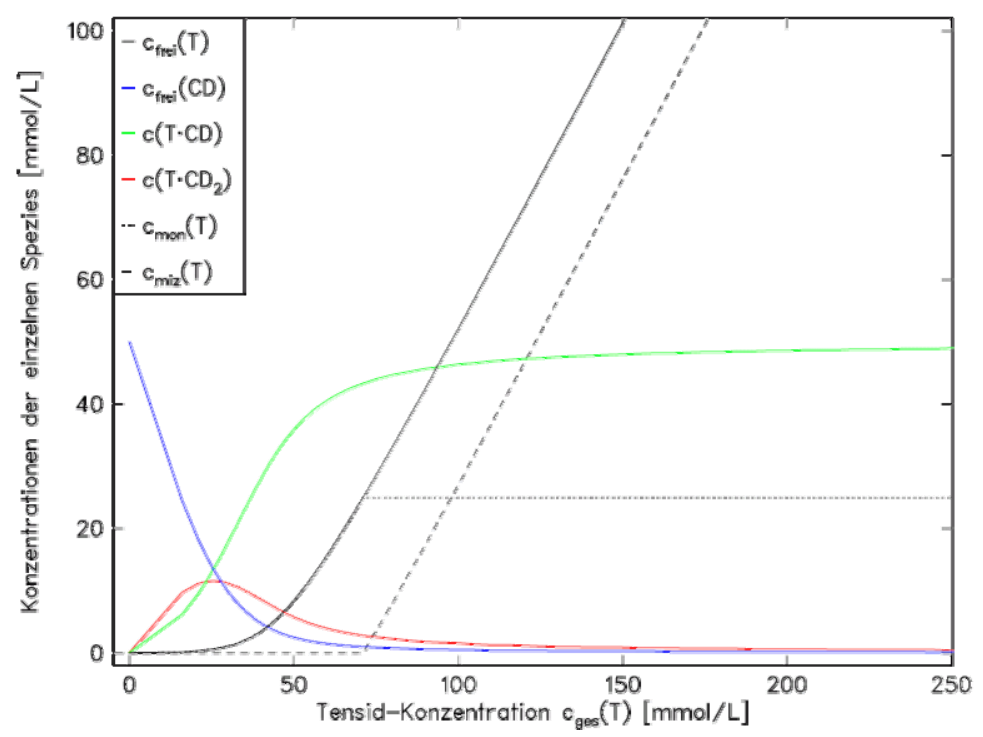

Abbildung 85: Verlauf der Konzentrationen der einzelnen Spezies in $\alpha-C D / C_{8} G_{1}-L o ̈ s u n g e n$. Daten errechnet aus Gl. (5.4) bis (5.10) $\mathrm{mit} K_{11}=1700 \mathrm{~L} / \mathrm{mol}, K_{21}=0, K_{12}=64 \mathrm{~L} / \mathrm{mol}$ und $c_{\text {ges }}(\mathrm{CD})=50 \mathrm{mmol} / \mathrm{L}$.

Es zeigt sich, dass die Verläufe insofern plausibel sind, als bei etwa $c_{\text {ges }}(\mathrm{T}) \approx 72 \mathrm{mmol} / \mathrm{L}$ die Konzentration der nicht komplexierten Tensidmoleküle gerade der $c m c$ von $\mathrm{C}_{8} \mathrm{G}_{1}$ entspricht. Diese Konzentration entspricht also der scheinbaren $c m c^{*}: c m c^{*} \approx 72 \mathrm{mmol} / \mathrm{L}$. Es kann nun bei der Analyse der Parameter der einzelnen Prozesse aus den Ultraschallabsorptionsspektren überprüft werden, ob sie mit den hier errechneten Konzentrationen plausibel zu erklären sind. 


\subsubsection{Monomeraustauschprozess}

Aus der im vorigen Abschnitt errechneten Konzentration freier, also nicht komplexierter Tensidmoleküle kann nun die reduzierte Konzentration gemäß der Gleichung $X_{\alpha-\mathrm{CD} / \mathrm{C} 8 \mathrm{G} 1}=\left(c_{f r e i}(\mathrm{~T})-c m c^{*}\right) / c m c_{\mathrm{C} 8 \mathrm{G} 1}$ berechnet werden. In Abbildung 86 sind die Relaxationsraten und in Abbildung 87 die Amplituden für den Monomeraustauschprozessterm der $\alpha-C D / C_{8} G_{1}$-Mischungen gegen die so erhaltene reduzierte Konzentration aufgetragen - jeweils zusammen mit den Daten der reinen $\mathrm{C}_{8} \mathrm{G}_{1}$-Lösungen.

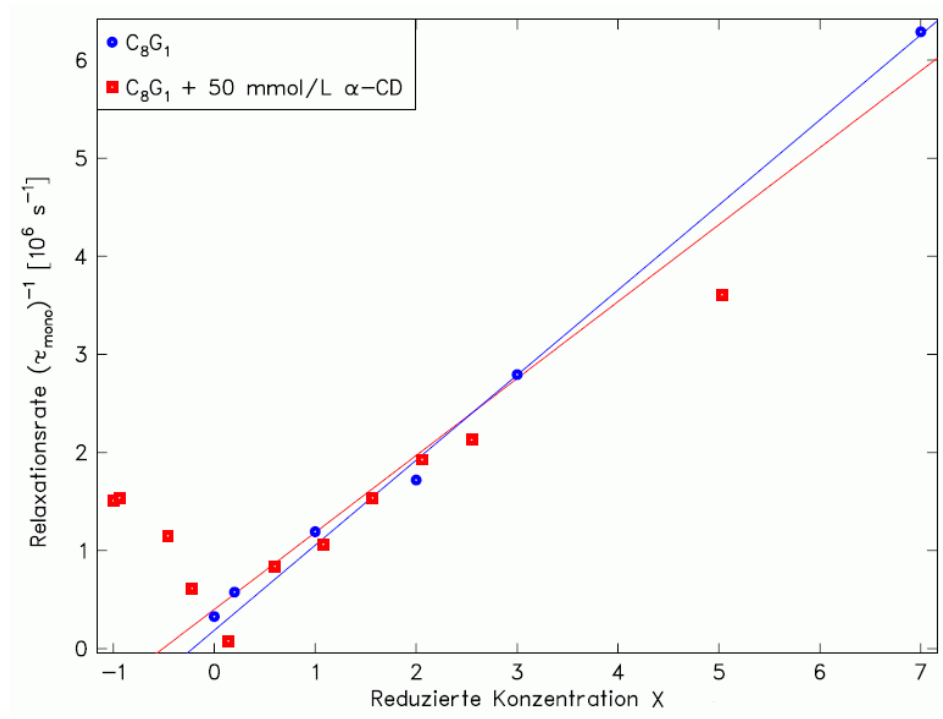

Abbildung 86: Relaxationsrate $\left(\tau_{M o n o}\right)^{-1}$ des Monomeraustauschprozesses der $\alpha-C D / C_{8} G_{1}-L o ̈ s u n g e n$ (rot) und der reinen $\mathbf{C}_{8} \mathbf{G}_{1}$-Lösungen (blau). Die durchgezogenen Linien entstammen der jeweiligen linearen Regression für die Daten bei $X_{\alpha-\mathrm{CD} / \mathrm{C} 8 \mathrm{G} 1}>0$.

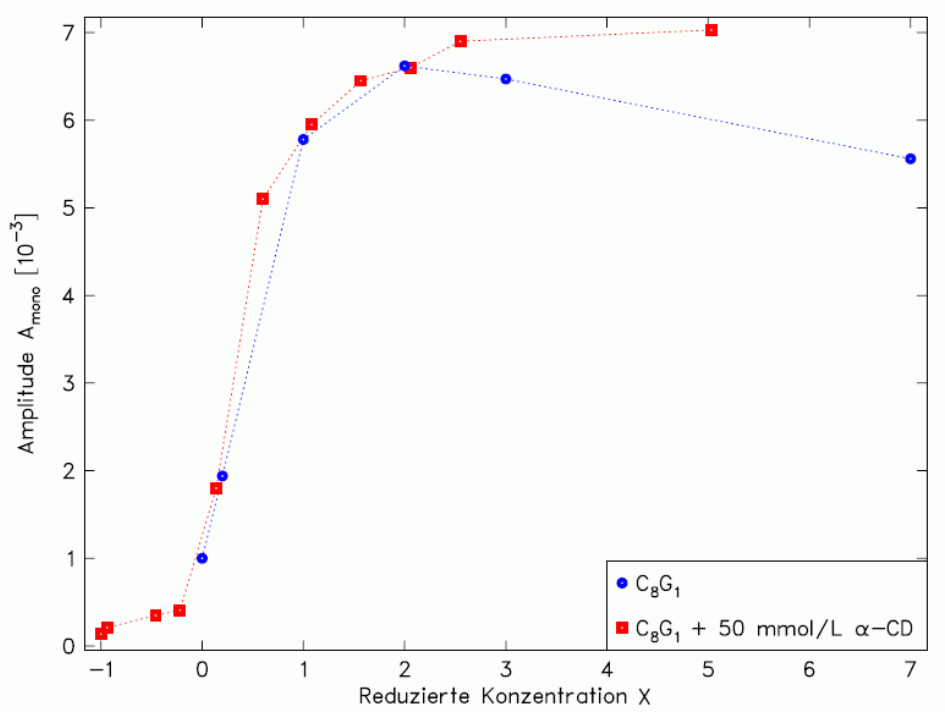

Abbildung 87: Amplitude $A_{M o n o}$ des Monomeraustauschprozess-Terms der $\alpha-C_{-} / C_{8} G_{1-}$ Lösungen(rot) und der reinen $\mathbf{C}_{8} \mathbf{G}_{1}$-Lösungen (blau). Die gestrichelten Linien dienen nur der Verdeutlichung der Verläufe.

Es zeigt sich, dass sowohl die Amplituden als auch die Relaxationsraten der beiden Systeme nach diesen Berechnungen sehr gut zueinander passen. Lediglich im Bereich höherer reduzierter Konzentrationen weichen die 
Verläufe voneinander ab. Dies liegt an dem beim System $\mathrm{C}_{8} \mathrm{G}_{1}$ auftretenden zweiten Monomeraustauschprozess. Interessanterweise weisen in diesem Bereich auch die Anpassungen der Ultraschallabsorptionsspektren der $\alpha$ $\mathrm{CD} / \mathrm{C}_{8} \mathrm{G}_{1}$-Mischungen gewisse systematische Abweichungen auf, so dass zu vermuten ist, dass die spezielle Mizellgeometrie von $\mathrm{C}_{8} \mathrm{G}_{1}$, die den zweiten Monomeraustauschprozess vermutlich verursacht, auch durch die Zugabe von Cyclodextrin kaum oder gar nicht beeinflusst wird. Allerdings wäre eine Anpassung mit einem weiteren Debye-Spektralterm im Falle der $\alpha-\mathrm{CD} / \mathrm{C}_{8} \mathrm{G}_{1}$-Lösungen zu unsicher, so dass hier darauf verzichtet werden soll.

Die lineare Regression des Verlaufes der Relaxationsrate über der reduzierten Konzentration liefert dennoch (unter der Annahme, dass die mittlere Aggregationszahl $\bar{m}$ sich durch die Zugabe von Cyclodextrin nicht ändert) ähnlich Werte für beide Systeme:

\begin{tabular}{l|r|r|r|r|r|r|c}
\hline \hline \multicolumn{1}{c|}{ Substanz } & $\begin{array}{c}c m c \\
{[\mathrm{mmol} / \mathrm{L}]}\end{array}$ & $\bar{m}$ & $\begin{array}{c}k_{b} / \sigma^{2} \\
{\left[10^{6} \mathrm{~s}^{-1}\right]}\end{array}$ & $\begin{array}{c}k_{b} / \bar{m} \\
{\left[10^{6} \mathrm{~s}^{-1}\right]}\end{array}$ & $\begin{array}{c}k_{b} \\
{\left[10^{6} \mathrm{~s}^{-1}\right]}\end{array}$ & $\begin{array}{c}k_{f} \\
{\left[10^{6} \mathrm{~L}_{\mathrm{mol}}{ }^{-1} \cdot \mathrm{s}^{-1}\right]}\end{array}$ & $\sigma$ \\
\hline \hline $\mathrm{C}_{8} \mathrm{G}_{1}$ & 25 & 80 & 0.29 & 0.85 & 68 & 2709 & 15.4 \\
$\mathrm{C}_{8} \mathrm{G}_{1}+50 \mathrm{mmol} / \mathrm{L} \alpha-\mathrm{CD}$ & $c m c^{*}=70$ & 80 & 0.39 & 0.78 & 62 & 2480 & 12.6 \\
\hline \hline
\end{tabular}

Tabelle 5.7: Parameter des Monomeraustauschprozesses der Systeme $C_{8} G_{1}$ und $\alpha-C D / C_{8} G_{1}$.

Weiterhin lässt sich aus den Amplituden des Monomeraustauschprozesses gemäß Gl. (3.7) das Reaktionsvolumen für das System $\alpha-C D / C_{8} G_{1}$ berechnen. Die so erhaltenen Werte $\Delta V=9.7 \mathrm{~mL} / \mathrm{mol}$ und $\Delta V / n_{C}=1.2 \mathrm{~mL} / \mathrm{mol}$ stimmen wiederum sehr gut mit denen von reinen $\mathrm{C}_{8} \mathrm{G}_{1}$-Lösungen überein.

Die guten Übereinstimmungen der Parameter weisen darauf hin, dass sowohl die Berechnung der Konzentration freier Tensidmoleküle im vorigen Abschnitt als auch die oben erwähnte Annahme, dass oberhalb der scheinbaren $c m c^{*}$ die Mizellkinetik nicht wesentlich durch die Anwesenheit der Cyclodextrin-Moleküle beeinflusst wird, gerechtfertigt sind. 


\subsubsection{Komplexbildungsprozess}

Die Zuordnung des zweiten auftretenden Relaxationsterms zu einer Komplexierung von $\alpha-C D$ und $C_{8} G_{1}$ lässt sich mit zwei Argumenten plausibel begründen. Zum einen weisen weder das Spektrum von reiner $\alpha$-CD-Lösung noch die Spektren von reinen $\mathrm{C}_{8} \mathrm{G}_{1}$-Lösungen einen Prozess auf, der (bei vergleichbaren $\mathrm{C}_{8} \mathrm{G}_{1}$-Konzentrationen) ähnliche Relaxationszeiten aufweist. Dementsprechend ist die Zuordnung dieses Prozess zu einer Wechselwirkung zwischen $\alpha-C D$ und $\mathrm{C}_{8} \mathrm{G}_{1}$ plausibel. Zum anderen ist der Verlauf der Relaxationsraten dieses Prozesses charakteristisch für einen Aggregations- oder Komplexierungsprozess, da die Relaxationsraten linear mit der $\mathrm{C}_{8} \mathrm{G}_{1}$-Konzentration ansteigen, wie in Abbildung 88 dargestellt. Die relative große Streuung der Werte sowie die sehr große Abweichung des Punktes bei $c_{\mathrm{C} 8 \mathrm{G} 1}=33 \mathrm{mmol} / \mathrm{L}$ lassen sich dadurch erklären, dass der Relaxationsterm in den Ultraschallspektren von den beiden benachbarten (Monomeraustauschprozess und Rotation der glykosidischen Bindung) teilweise deutlich überdeckt wird (siehe Abbildung 55).

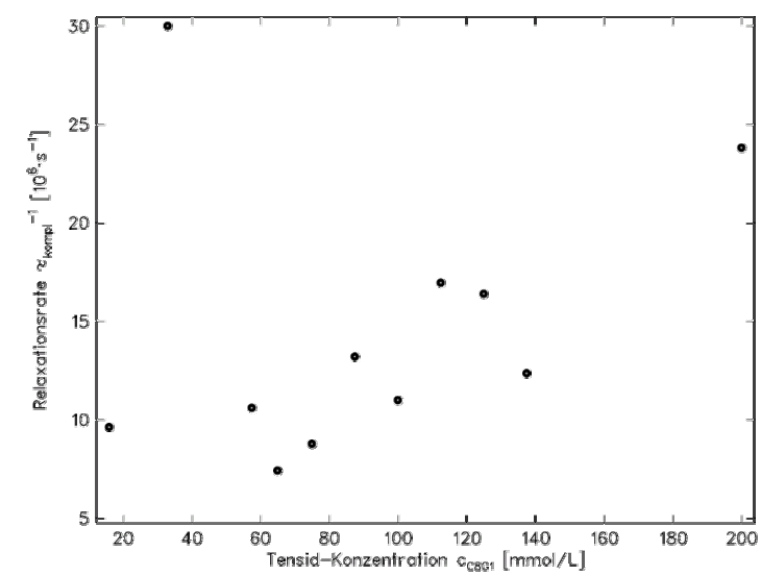

\section{Abbildung 88: Relaxationsrate $\left(\tau_{\text {kompl }}\right)^{-1}$ des Komplexbildungs-Prozesses von $\alpha-C D$ und $C_{8} G_{1}$ in $A b-$ hängigkeit von der $\mathrm{C}_{8} \mathrm{G}_{1}$-Konzentration.}

Unklar ist zunächst, welcher der verschiedenen in Tabelle 5.6 aufgelisteten Komplexbildungsreaktionen dieser Term zuzuordnen ist. Um diese Frage beantworten zu können, werden die stöchiometrischen Faktoren für die einzelnen Reaktionen gemäß Gl. (2.26) berechnet:

$$
\begin{aligned}
& \Gamma_{11}=\left(c_{\text {frei }}^{-1}(\mathrm{~T})+c_{\text {frei }}^{-1}(\mathrm{CD})+c^{-1}(\mathrm{~T} \circ \mathrm{CD})\right)^{-1} \\
& \Gamma_{21}=\left(c_{\text {frei }}^{-1}(\mathrm{~T})+c^{-1}(\mathrm{~T} \circ \mathrm{CD})+c^{-1}\left(\mathrm{~T}_{2} \circ \mathrm{CD}\right)\right)^{-1} \\
& \Gamma_{12}=\left(c_{\text {frei }}^{-1}(\mathrm{CD})+c^{-1}(\mathrm{~T} \circ \mathrm{CD})+c^{-1}\left(\mathrm{~T} \circ \mathrm{CD}_{2}\right)\right)^{-1}
\end{aligned}
$$

In Abbildung 89 sind die Prozessamplituden $\tilde{A}_{k o m p l}=\Gamma \cdot(\Delta V)^{2}$ gegen die Tensidkonzentration aufgetragen. Zusätzlich sind die Verläufe von $\Gamma_{11} \cdot(\Delta V)^{2}, \Gamma_{21} \cdot(\Delta V)^{2}$ und $\Gamma_{12} \cdot(\Delta V)^{2}$ dargestellt. Dabei ist ein Reaktionsvolumen $\Delta V=3$ $\mathrm{mL} / \mathrm{mol}$ zunächst als willkürlicher Skalierungsfaktor bereits so gewählt, dass die theoretischen Verläufe möglichst gut zu den Messpunkten passen. Es zeigt sich allerdings deutlich, dass der charakteristische Verlauf der berechneten Prozessamplituden für die Bildung von $\mathrm{T} \cdot \mathrm{CD}-\mathrm{Komplexen}$ und $\mathrm{T}{ }^{\circ} \mathrm{CD}_{2}$-Komplexen die Messpunkte 
nicht befriedigend wiedergeben kann. Daher wurde zusätzlich eine Berechnung durchgeführt, bei der als Gleichgewichtskonstante für die Bildung von $\mathrm{T}_{2}{ }^{\circ} \mathrm{CD}$-Komplexen nicht $K_{21}=0$, wie in [117] angegeben, sondern der zunächst willkürliche kleine Wert $K_{21}=1 \mathrm{~L} / \mathrm{mol}$ benutzt wurde. Da dieser Wert wesentlich kleiner als $K_{12}$ und $K_{11}$ ist, ändern sich dadurch die in Abbildung 85 dargestellten Verläufe der Konzentrationen der anderen Spezies nur sehr geringfügig, wie auch die berechneten Verläufe der Prozessamplituden in Abbildung 89. Es ist offensichtlich, dass die Charakteristik des Verlaufes für $\mathrm{T}_{2}{ }^{\circ} \mathrm{CD}-$ Komplexe als einzige eine Ähnlichkeit mit jener der Messpunkte aufweist. Weiterhin lässt sich zeigen, dass die Charakteristik der beiden anderen Verläufe auch durch Variationen der Gleichgewichtskonstanten $K_{11}$ und $K_{12}$ nicht soweit geändert werden kann, dass sie die Messpunkte zufriedenstellend beschreiben. Daher muss der Verlauf der Messpunkte als Indiz dafür gewertet werden, dass entgegen der Vermutungen in der Literatur auch $\mathrm{T}_{2}{ }^{\circ} \mathrm{CD}$-Komplexe auftreten können. Der Wert der entsprechenden Gleichgewichtskonstanten $K_{21}$ kann allerdings hier nicht sehr genau ermittelt werden - auch Anpassungen mit $K_{21}=0.1 \mathrm{~L} / \mathrm{mol}$ bis $K_{21}=5 \mathrm{~L} / \mathrm{mol}$ ergeben ähnliche Verläufe wie den für $K_{21}=1 \mathrm{~L} / \mathrm{mol}$. Daher kann auch das oben ermittelte Reaktionsvolumen von $\Delta V=3 \mathrm{~mL} / \mathrm{mol}$ nur als Schätzwert betrachtet werden, denn bei $K_{21}=0.1 \mathrm{~L} / \mathrm{mol}$ ergibt sich die beste Anpassung mit $\Delta V=10 \mathrm{~mL} / \mathrm{mol}$, bei $K_{21}=5 \mathrm{~L} / \mathrm{mol} \mathrm{mit} \Delta V=2 \mathrm{~mL} / \mathrm{mol}$. Die Größenordnung erscheint aber in jedem Fall plausibel, da das Reaktionsvolumen des Monomeraustauschprozesses bei $\mathrm{C}_{8} \mathrm{G}_{1}$-Lösungen etwa $\Delta V=10 \mathrm{~mL} / \mathrm{mol}$ (siehe Kapitel 5.1) beträgt und als Reaktionsvolumen einer Komplexbildung von $\mathrm{C}_{8} \mathrm{G}_{1}$ mit Cyclodextrin ein gewisser Teil davon zu erwarten ist.

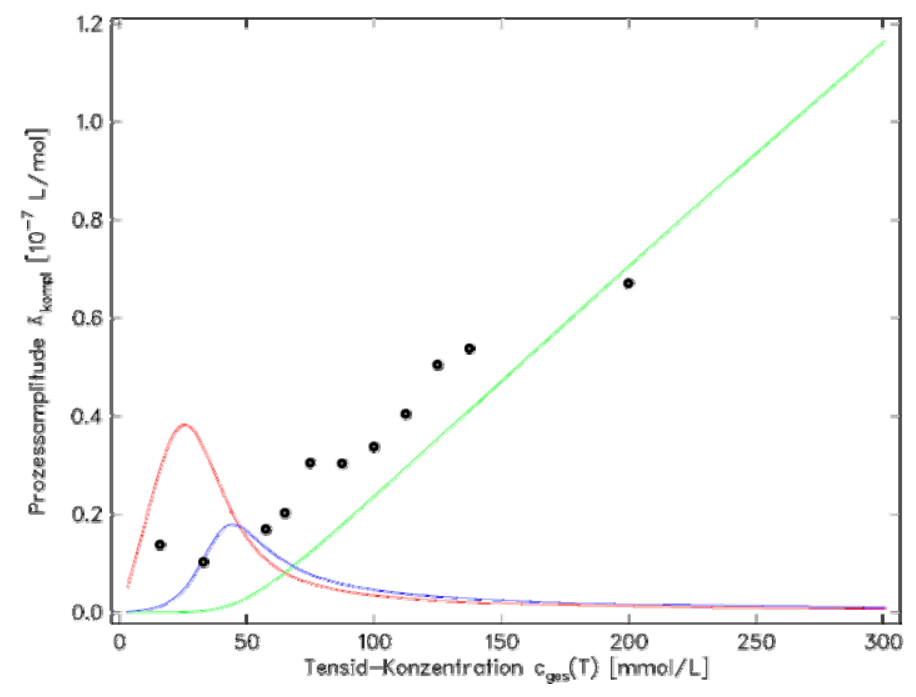

Abbildung 89: Prozessamplitude $\boldsymbol{A}_{\text {kompl }}$ des Komplexbildungs-Prozesses über der Tensidkonzentration. $\mathrm{Zu}$ den Messpunkten sind die theoretischen Verläufe für die Bildung von $\mathrm{T}{ }^{\circ} \mathrm{CD}-\mathrm{Komplexen}$ (blau), $\mathrm{T} \circ \mathrm{CD}_{2}$-Komplexen (rot) und $\mathrm{T}_{2}{ }^{\circ} \mathrm{CD}-$ Komplexen (grün) aufgetragen. Letzterer ist berechnet mit $K_{21}=1$ $\mathrm{L} / \mathrm{mol}$, für alle drei Verläufe wurde $\Delta V=3 \mathrm{~mL} / \mathrm{mol}$ angenommen (siehe Text).

Zur Überprüfung der getroffenen Zuordnung des Prozesses zur Bildung von $\mathrm{T}_{2}{ }^{\circ} \mathrm{CD}-$ Komplexen kann nun für alle drei Komplexbildungsprozesse gemäß Gl. (2.27) der qualitative Verlauf der Relaxationsraten überprüft und zudem quantitativ analysiert werden. Nach Gl. (2.27) gilt für die obigen Prozesse (mit $K_{11}=k^{11}{ }_{f} / k^{11}{ }_{b}, K_{12}=k^{12}{ }_{f} / k^{12}{ }_{b}$ und $K_{21}=k_{f}^{21} / k^{21}{ }_{b}$ ): 


$$
\begin{aligned}
\tau_{11}{ }^{-1} & =k_{f}^{11} \cdot\left(c_{\text {frei }}(\mathrm{T})+c_{\text {frei }}(\mathrm{CD})\right)+k_{b}^{11} \\
& =k_{f}^{11} \cdot\left[c_{\text {frei }}(\mathrm{T})+c_{\text {frei }}(\mathrm{CD})+\left(K_{11}\right)^{-1}\right] \\
\tau_{21}{ }^{-1} & =k_{f}^{21} \cdot\left(c_{\text {frei }}(\mathrm{T})+c(\mathrm{~T} \circ \mathrm{CD})\right)+k_{b}^{21} \\
& =k_{f}^{21} \cdot\left[c_{\text {frei }}(\mathrm{T})+K_{11} \cdot c_{f r e i}(\mathrm{~T}) \cdot c_{f r e i}(\mathrm{CD})+\frac{1}{K_{21}}\right] \\
\tau_{12}{ }^{-1} & =k_{f}^{12} \cdot\left(c_{\text {frei }}(\mathrm{CD})+c(\mathrm{~T} \circ \mathrm{CD})\right)+k_{b}^{12} \\
& =k_{f}^{12} \cdot\left[c_{\text {frei }}(\mathrm{CD})+K_{11} \cdot c_{\text {frei }}(\mathrm{CD}) \cdot c_{\text {frei }}(\mathrm{T})+\cdot \frac{1}{K_{12}}\right]
\end{aligned}
$$

Somit bleibt also wiederum nur ein freier Parameter, nämlich die jeweilige Reaktionskonstante der Komplexbildung $k_{f}$, bei der Anpassung der theoretischen Verläufe an die Relaxationszeiten zu bestimmen. In Abbildung 90 ist die jeweils beste Anpassung dargestellt. Im Gegensatz zu den Verläufen der Prozessamplituden weisen hier im Grunde alle drei theoretischen Verläufe (insbesondere $\tau_{11}{ }^{-1}$ und $\tau_{21}{ }^{-1}$ ) eine etwa gleich gute Übereinstimmung mit den Messpunkten auf. Die jeweiligen Anpassungsparameter $k_{f}^{11}=2 \cdot 10^{8} \mathrm{~L}^{\prime} \mathrm{mol}^{-1} \cdot \mathrm{s}^{-1}, k_{f}^{21}=2.5 \cdot 10^{8} \mathrm{~L} \cdot \mathrm{mol}^{-1} \cdot \mathrm{s}^{-1}$ und $k_{f}^{12}=1.3 \cdot 10^{7} \mathrm{~L} \mathrm{~mol}^{-1} \cdot \mathrm{s}^{-1}$ erscheinen dabei ebenfalls alle plausibel - sie sind aber, wie auch die genaue Steigung der theoretischen Verläufe in Abbildung 90, stark von der jeweiligen Gleichgewichtskonstanten abhängig. Insbesondere führt auch die Annahme eines größeren Wertes von $K_{21}$ zu einem steileren Verlauf der Relaxationsrate $\left(\tau_{21}\right)^{-1}$.

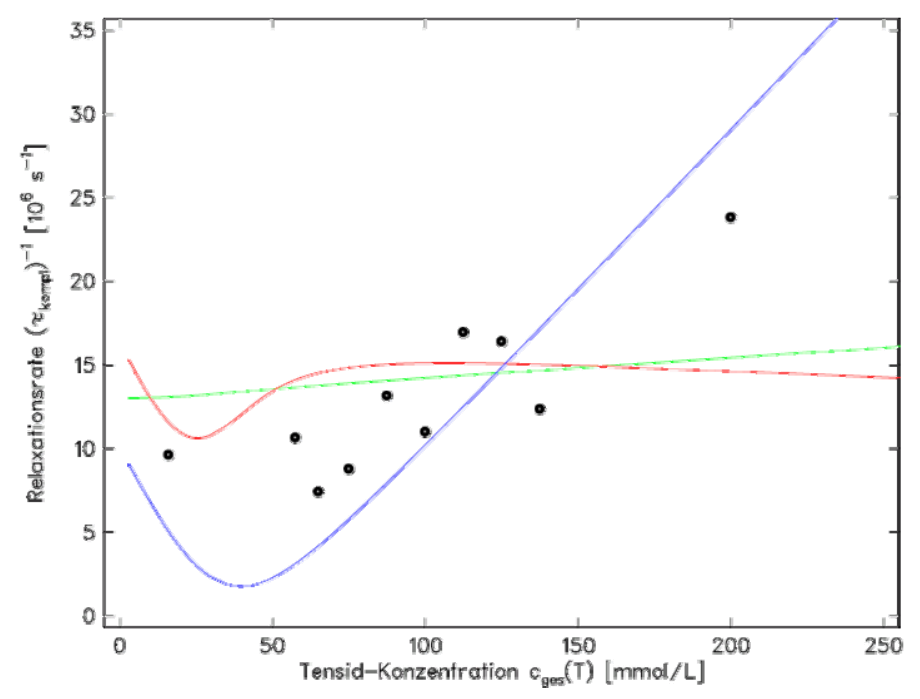

Abbildung 90: Relaxationsrate $\left(\tau_{\text {kompl }}\right)^{-1}$ des Komplexbildungsprozesses. Zusätzlich zu den Messpunkten ist der theoretische Verlauf für die Bildung von T॰CD-Komplexen (blau, mit Anpassungsparameter

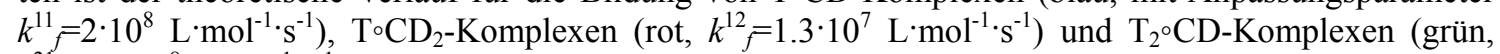

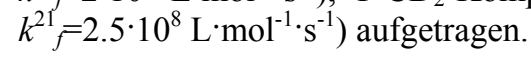

Die obigen Verläufe verlangen nicht wie die der Prozessamplituden zwingend eine Zuordnung des Relaxationsterms zur Bildung von $\mathrm{T}_{2}{ }^{\circ} \mathrm{CD}$-Komplexen, allerdings schließen sie diese Zuordnung auch nicht aus. Weiter- 
hin kann wegen der sehr ähnlichen Verläufe der Relaxationsraten $\tau_{11}{ }^{-1}$ und $\tau_{21}{ }^{-1}$ auch nicht ausgeschlossen werden, dass es sich bei dem gefundenen Prozess möglicherweise um eine Überlagerung zweier Anteile handelt auch der Verlauf der Prozessamplituden wäre mit einem dementsprechenden theoretischen Verlauf von $\Gamma_{11} \cdot\left(\Delta V_{11}\right)^{2}+\Gamma_{21} \cdot\left(\Delta V_{21}\right)^{2}$ vermutlich gut zu beschreiben. 


\subsubsection{Rotation der glykosidischen Bindung}

Der dritte in den $\alpha-C D / C_{8} G_{1}$-Spektren auftretende Term ist wieder der teilweisen Rotation der glykosidischen Bindungen zuzuordnen. Da sowohl bisherige Messungen an $\alpha$-Cyclodextrin-Lösungen einen Prozess in diesem Zeitbereich aufweisen ( $\tau=21 \mathrm{~ns}$ für reines $\alpha$-Cyclodextrin, $100 \mathrm{mmol} / \mathrm{L}, \tau=(7.5 \pm 1.5) \mathrm{ns}$ bei Zugabe von KI, [49]) als auch die Messungen an reinen $\mathrm{C}_{8} \mathrm{G}_{1}$-Lösungen $(\tau=(11.5 \pm 4.5)$ ns, siehe Tabelle 4.5), ist davon auszugehen, dass es sich bei diesem Prozess um eine Überlagerung des Anteils der Cyclodextrin-Moleküle und des Anteils der $\mathrm{C}_{8} \mathrm{G}_{1}$-Moleküle handelt. Weiterhin ist davon auszugehen, dass freie $\mathrm{C}_{8} \mathrm{G}_{1}$-Moleküle, komplexierte $\mathrm{C}_{8} \mathrm{G}_{1}$ Moleküle, freie Cyclodextrin-Moleküle, komplexierte Cyclodextrin-Moleküle und in Mizellen aggregierte $\mathrm{C}_{8} \mathrm{G}_{1}$ Moleküle unterschiedliche Beiträge zur Gesamt-Amplitude leisten.

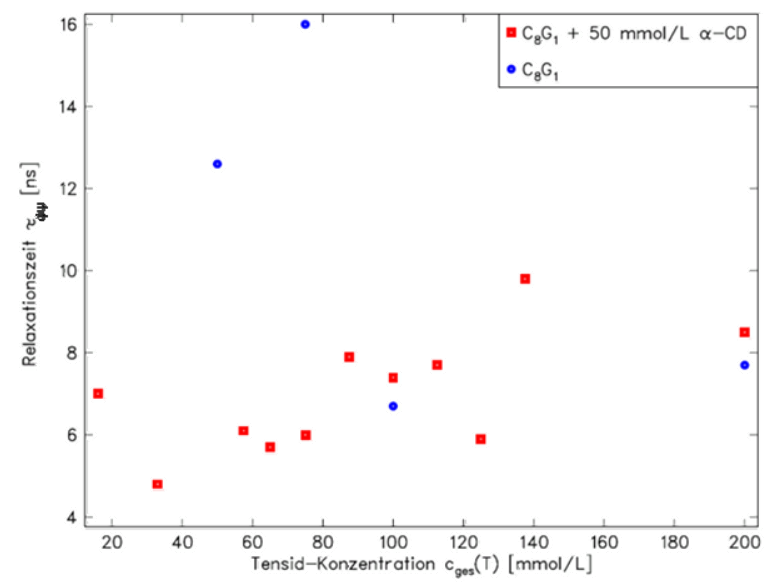

\section{Abbildung 91: Relaxationszeit $\tau_{\varphi \psi}$ des Rotationsterms der glykosidischen Bindung bei $\alpha-C_{D} / C_{8} G_{1-}$ Mischungen (rot) und reinen $\mathrm{C}_{8} \mathrm{G}_{1}$-Lösungen (blau).}

Während die Relaxationszeiten im Rahmen der Fehler gut mit denen der reinen $\mathrm{C}_{8} \mathrm{G}_{1}$-Lösungen übereinstimmen, aber keine signifikante Abhängigkeit von der Tensidkonzentration aufweisen (siehe Abbildung 91), kann aus der Konzentrationsabhängigkeit der Prozessamplituden wieder eine grobe quantitative Analyse vorgenommen werden. Dafür werden folgende Annahmen getroffen:

- Die Gesamt-Prozessamplitude setzt sich additiv aus den einzelnen Anteilen zusammen: $\tilde{A}_{\text {ges }}=\tilde{A}_{\mathrm{CD}}{ }^{f r e i}+\tilde{A}_{\mathrm{T}}^{\text {frei }}+\tilde{A}_{\mathrm{CD}}{ }^{k}+\tilde{A}_{\mathrm{T}}{ }^{k}$. Hierbei steht der Index,$k^{k}$ für alle Komplexe, die zur Reduzierung der Parameter nicht unterschieden werden.

- Um einen weiteren Parameter zu eliminieren, wird hier davon ausgegangen, dass bei den Komplexen $\mathrm{T} \circ \mathrm{CD}$, $\mathrm{T}{ }^{\circ} \mathrm{CD}_{2}$ und $\mathrm{T}_{2}{ }^{\circ} \mathrm{CD}$ das/die Cyclodextrin-Molekül/e wesentlich stärker zur Prozessamplitude beiträgt bzw. beitragen als das/die Tensid-Molekül/e. Dadurch können die Prozessamplituden für die Komplexe zusammengefasst werden: $\tilde{A}^{k} \equiv \tilde{A}_{\mathrm{CD}}{ }^{k}+\tilde{A}_{\mathrm{T}}{ }^{k}$. Dieser Anteil ist dann proportional zur Konzentration der Komplexe: $\tilde{A}^{k}=\Sigma_{k} \cdot\left(\Delta V_{k}\right)^{2} \cdot\left(c(\mathrm{~T} \circ \mathrm{CD})+c\left(\mathrm{~T}_{2}{ }^{\circ} \mathrm{CD}\right)+c\left(\mathrm{~T} \circ \mathrm{CD}_{2}\right)\right)$.

- Die Prozessamplitude der Rotation der glykosidischen Bindung von reinem $\alpha$-Cyclodextrin kann aus dem Literaturwert für eine Lösung mit $100 \mathrm{mmol} / \mathrm{L}, \tilde{A}(100 \mathrm{mmol} / \mathrm{L})=4.67 \cdot 10^{-8} \mathrm{~L} / \mathrm{mol}$ ([49]), für eine beliebige Konzentration extrapoliert werden $\mathrm{zu} \tilde{A}_{\mathrm{CD}}{ }^{f r e i}(c)=(c / 100 \mathrm{mmol} / \mathrm{L}) \cdot \tilde{A}(100 \mathrm{mmol} / \mathrm{L})=c \cdot 4.67 \cdot 10^{-7} \mathrm{~L}^{2} / \mathrm{mol}^{2}$. 
- Die Prozessamplitude der nicht komplexierten $\mathrm{C}_{8} \mathrm{G}_{1}-$ Moleküle entspricht der einer entsprechenden Konzentration aus den reinen $\mathrm{C}_{8} \mathrm{G}_{1}$-Lösungen. Eine entsprechende Interpolation der Daten der reinen Lösungen ergibt $\tilde{A}_{\mathrm{T}}^{\text {frei }}(c)=1.81 \cdot 10^{-6} \mathrm{~L}^{2} / \mathrm{mol}^{2} \cdot c-6.54 \cdot 10^{-8} \mathrm{~L} / \mathrm{mol}$.

Damit gilt für die Prozessamplituden der $\alpha-C D / C_{8} G_{1}-$ Mischungen:

$$
\begin{aligned}
\tilde{A}\left(c_{\text {ges }}(\mathrm{T})\right)= & \tilde{A}_{\mathrm{CD}}{ }^{\text {frei }}\left(c_{\text {ges }}(\mathrm{T})\right)+\tilde{A}_{\mathrm{T}}^{\text {frei }}\left(c_{\text {ges }}(\mathrm{T})\right)+\tilde{A}^{k}\left(c_{\text {ges }}(\mathrm{T})\right) \\
= & 4.67 \cdot 10^{-7} \frac{\mathrm{L}^{2}}{\mathrm{~mol}^{2}} \cdot c_{\mathrm{CD}}{ }^{\text {frei }}+1.81 \cdot 10^{-6} \frac{\mathrm{L}^{2}}{\mathrm{~mol}^{2}} \cdot c_{\mathrm{T}}^{\text {frei }} \\
& -6.54 \cdot 10^{-8} \frac{\mathrm{L}}{\mathrm{mol}}+\Sigma_{k} \cdot\left(\Delta V_{k}\right)^{2} \cdot\left(c_{11}+c_{21}+c_{12}\right)
\end{aligned}
$$

Da die einzelnen Konzentrationen $c_{\text {frei }}(\mathrm{CD}), c_{f r e i}(\mathrm{~T})$ und $\left(c(\mathrm{~T} \circ \mathrm{CD})+c\left(\mathrm{~T}_{2}{ }^{\circ} \mathrm{CD}\right)+c\left(\mathrm{~T}^{\circ} \mathrm{CD}_{2}\right)\right)=c_{\text {ges }}(\mathrm{CD})-c_{f r e i}(\mathrm{CD})$ bekannt sind, tritt bei einer Anpassung dieser Form an die Prozessamplituden wiederum nur ein freier Parameter, nämlich das Produkt $\Sigma_{k} \cdot\left(\Delta V_{k}\right)^{2}$, auf. In Abbildung 92 ist der Verlauf der Prozessamplituden zusammen mit dem Graphen von Gl. (5.17) aufgetragen, wobei der aus der Regressionsanalyse folgende Parameter $\Sigma_{k} \cdot\left(\Delta V_{k}\right)^{2}=25 \cdot 10^{-7}$ $\mathrm{L}^{2} / \mathrm{mol}^{2}$ verwendet wurde.

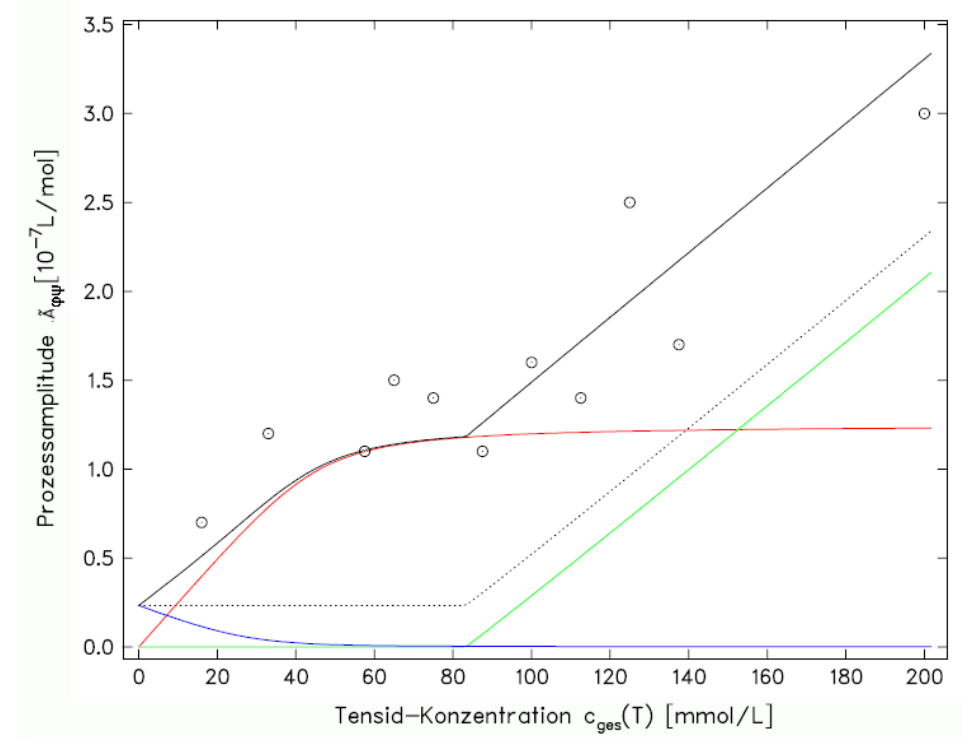

Abbildung 92: Prozessamplitude $\tilde{A}$ des der Rotation der glykosidischen Bindung zugeordneten Terms. Die schwarze durchgezogene Linie gibt die Funktion gemäß Gl. (5.17) mit dem Anpassungsparameter $\Sigma_{k} \cdot\left(\Delta V_{k}\right)^{2}=25 \cdot 10^{-7} \mathrm{~L}^{2} / \mathrm{mol}^{2}$ wieder; die bunten durchgezogenen Linien entsprechen den einzelnen Anteilen: $\tilde{A}_{\mathrm{CD}}{ }^{\text {frei }}$ (blau); $\tilde{A}_{\mathrm{T}}{ }^{\text {frei }}$ (grün); $\tilde{A}_{\mathrm{CD}}{ }^{k}$ (rot). Zum Vergleich ist zudem der Verlauf eingetragen, der sich aus einer rein additiven Überlagerung der Anteile von $50 \mathrm{mmol} / \mathrm{L} \alpha-\mathrm{CD}$ und der jeweiligen TensidKonzentration ergeben würde (gepunktete schwarze Linie), also ohne Berücksichtigung von Wechselwirkungen.

Obwohl die Anpassung die Punkte offenbar sehr gut beschreibt, muss hier darauf hingewiesen werden, dass es sich bei dem resultierenden Wert für den Anpassungsparameter $\Sigma_{k} \cdot\left(\Delta V_{k}\right)^{2}=25 \cdot 10^{-7} \mathrm{~L}^{2} / \mathrm{mol}^{2}$ nur um eine sehr grobe Abschätzung handelt, da zum einen für das zugrunde liegende Modell relativ viele Annahmen getroffen werden mussten, um die Anzahl der freien Parameter zu reduzieren, und sich zum anderen die Anpassung als sehr unempfindlich gegenüber Variation des Anpassungsparameters erweist. Dennoch kann zumindest ein Vergleich der Größenordnungen des Produktes $\Sigma \cdot(\Delta V)^{2}$ für unterschiedliche Moleküle vorgenommen werden. Während die 
Werte für die Glukose-Alkyl-Bindung der Alkylglykoside von $\Sigma \cdot(\Delta V)^{2} \approx 0.5 \cdot 10^{-7} \mathrm{~L}^{2} / \mathrm{mol}^{2}$ im monomeren Zustand auf $\Sigma \cdot(\Delta V)^{2} \approx 16 \cdot 10^{-7} \mathrm{~L}^{2} / \mathrm{mol}^{2}$ im mizellaren Zustand anwachsen (siehe Tabelle 5.4), ergeben sich in dieser Arbeit die Werte für Glukose-Glukose-Bindungen von $\Sigma \cdot(\Delta V)^{2} \approx 2 \cdot 10^{-7} \mathrm{~L}^{2} / \mathrm{mol}^{2}$ bei Maltose, $\Sigma \cdot(\Delta V)^{2} \approx 5 \cdot 10^{-7} \mathrm{~L}^{2} / \mathrm{mol}^{2}$ bei unkomplexiertem Cyclodextrin (Extrapolation, siehe oben) und $\Sigma \cdot(\Delta V)^{2} \approx 25 \cdot 10^{-7} \mathrm{~L}^{2} / \mathrm{mol}^{2}$ bei komplexiertem Cyclodextrin. Es zeigt sich also, dass auch die Cyclodextrin-Moleküle eine deutliche Beeinflussung der Konformationskinetik der glykosidischen Bindung in Form eines signifikanten Anstiegs des Produktes $\Sigma \cdot(\Delta V)^{2}$ durch die sterischen Wechselwirkungen mit den Tensid-Molekülen erfahren.

Eine genauere Deutung dieses Effektes ist allerdings nicht ohne weitere Messungen möglich. In einem detaillierteren Modell müssten so zum Beispiel für die verschiedenen Komplexe unterschiedliche Beiträge angenommen bzw. bestimmt werden - so können zum Beispiel in $\mathrm{T}^{\circ} \mathrm{CD}_{2}$-Komplexen zwischen den beiden CyclodextrinMolekülen Wasserstoffbrückenbindungen ausgebildet werden (siehe Abbildung 93), die die Konformationskinetik der Rotation der glykosidischen Bindung sicherlich deutlich beeinflussen, so dass für diese Komplexe ein anderer Beitrag zu erwarten ist als für $\mathrm{T} \cdot \mathrm{CD}$ - und $\mathrm{T}_{2}{ }^{\circ} \mathrm{CD}$-Komplexe.

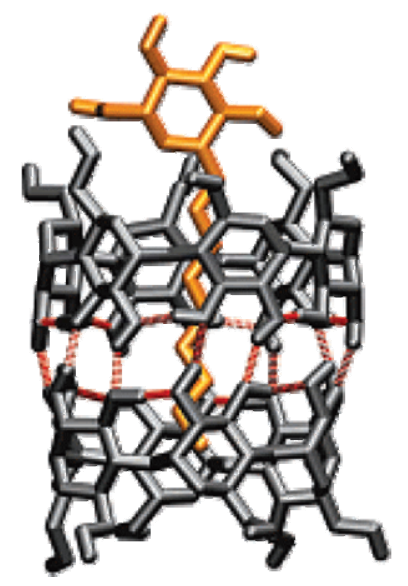

Abbildung 93: Mögliche Wasserstoffbrückenbindungen (rot) zwischen den Cyclodextrin-Molekülen (grau) in einem $\mathbf{T} \circ \mathbf{C D}_{2}$-Komplex. Grafik entnommen aus [117]. 


\subsubsection{Rotation der exozyklischen Gruppe}

Der hochfrequente Prozess in den $\alpha-C D / C_{8} G_{1}$-Spektren kann wieder eindeutig der Rotation der exozyklischen Gruppe zugeordnet werden, da die Relaxationszeiten alle im Bereich von 1.5 $\pm 0.5 \mathrm{~ns}$ liegen, wie auch jene der Glukose, Maltose und der Alkylglykoside. Da die Relaxationszeit bei den Anpassrechnungen der Ultraschallabsorptionsspektren der $\alpha-C D / C_{8} G_{1}$-Mischungen in einigen Fällen auf $\tau=2$ ns festgehalten werden musste, wird hier auf eine Analyse der Relaxationszeiten verzichtet. Stattdessen kann wiederum eine qualitative und (mit einigen Annahmen) eine quantitative Analyse der Prozessamplituden vorgenommen werden.

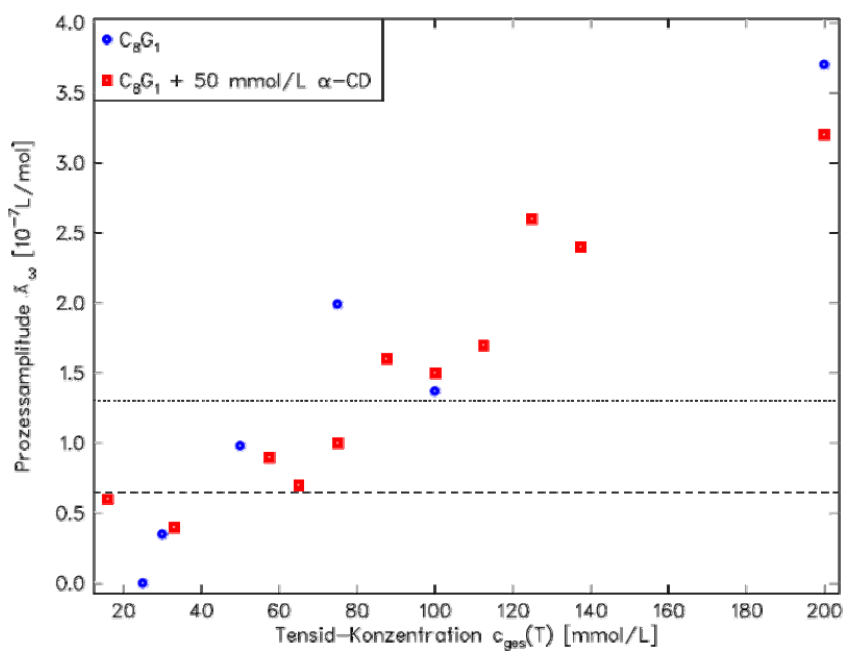

Abbildung 94: Prozessamplituden $\tilde{A_{\omega}}$ des der exozyklischen Rotation zugeordneten Terms. Außer den Werten der $\alpha$-CD/C $\mathrm{C}_{8} \mathrm{G}_{1}$-Mischungen (rot) und der reinen $\mathrm{C}_{8} \mathrm{G}_{1}$-Lösungen (blau) ist auch der Messwert für eine reine $\alpha$-Cyclodextrin-Lösung der Konzentration $100 \mathrm{mmol} / \mathrm{L}$ aus [49] eingetragen (gepunktete Linie) und ein daraus interpolierter Wert für $50 \mathrm{mmol} / \mathrm{L}$ (gestrichelte Linie).

Die in Abbildung 94 aufgetragenen Prozessamplituden $\tilde{A}_{\omega}$ der $\alpha-C D / C_{8} G_{1}$-Mischungen zeigen im Rahmen der Fehler eine sehr gute Übereinstimmung mit denen der reinen $\mathrm{C}_{8} \mathrm{G}_{1}$-Lösungen. Dies kann als deutlicher Beleg für eine Interaktion der verschiedenen Moleküle miteinander gewertet werden; denn wenn sich die einzelnen Molekülsorten nicht beeinflussen würden, wäre ein additives Verhalten der Prozessamplituden zu erwarten - die Werte der $\alpha-C D / C_{8} G_{1}$-Mischungen müssten dann um den Wert der reinen Cyclodextrin-Lösungen, also um etwa $0.65 \cdot 10^{-7} \mathrm{~L} / \mathrm{mol}$, höher liegen. Dass sterische Wechselwirkungen mit Nachbarmolekülen einen deutlichen Einfluss auf die Prozessamplitude des Terms der Rotation der exozyklischen Gruppe haben, wurde bereits in Kapitel 1.1 gezeigt und diskutiert, allerdings zeigt ein Vergleich der Daten von Glukose, Maltose und $\alpha$-Cyclodextrin, dass auch die unterschiedlichen Gruppen eines Moleküls deutlichen Einfluss aufeinander haben: Da Maltose zwei exozyklische Gruppen besitzt und $\alpha$-Cyclodextrin sogar sechs, wäre ohne Wechselwirkungen zu erwarten, dass die skalierte Prozessamplitude für beide Substanzen zweimal bzw. sechsmal größer ist als die der Glukose. Ein Vergleich der Werte von Glukose $\left(\tilde{A} / c=\Sigma \cdot(\Delta V)^{2} \approx 6.2 \cdot 10^{-7} \mathrm{~L}^{2} / \mathrm{mol}^{2}\right)$, Maltose $\left(\tilde{A} / c=\Sigma \cdot(\Delta V)^{2} \approx 7.4 \cdot 10^{-7} \mathrm{~L}^{2} / \mathrm{mol}^{2}\right)$ und $\alpha$-Cyclodextrin $\left(\tilde{A} / c=\Sigma \cdot(\Delta V)^{2} \approx 13.1 \cdot 10^{-7} \mathrm{~L}^{2} / \mathrm{mol}^{2}\right)$ zeigt, dass die skalierte Prozessamplitude zwar deutlich mit der Anzahl der exozyklischen Gruppen pro Moleküle ansteigt, aber offenbar keineswegs proportional.

Vor diesem Hintergrund erscheint es durchaus plausibel, dass sowohl die Rotation der exozyklischen Gruppe eines Alkylglykosid-Moleküls bei Eintritt in ein Cyclodextrin-Molekül stark eingeschränkt wird als auch die 
Rotation der exozyklischen Gruppen des Cyclodextrin-Moleküles selber (siehe auch Abbildung 93). Eine quantitative Analyse dieser gegenseitigen Beeinflussungen ist nicht möglich, da hierfür zu viele Annahmen getroffen werden müssten. So ist z.B. selbst bei genauer Kenntnis der Konzentrationen der einzelnen Komplextypen $\left(\mathrm{T} \circ \mathrm{CD}, \mathrm{T}_{2}{ }^{\circ} \mathrm{CD}, \mathrm{T} \circ \mathrm{CD}_{2}\right)$ nicht bekannt, in welchen Verhältnissen die Komplexe mit unterschiedlicher Orientierung der Moleküle zueinander (also z.B. $\mathrm{T}(\uparrow){ }^{\circ} \mathrm{CD}(\uparrow)$ und $\mathrm{T}(\downarrow){ }^{\circ} \mathrm{CD}(\uparrow)$ für $\mathrm{T} \circ \mathrm{CD}$, siehe Tabelle 5.6) auftreten. Diese Orientierung der Moleküle zueinander hat allerdings sicherlich evidenten Einfluss auf die Frage der gegenseitigen Beeinflussung der Rotationskinetik der exozyklischen Gruppe. Um dennoch ein quantitatives Maß für die Beeinflussung der Rotation der exozyklischen Gruppe durch sterische Behinderungen zu haben, sind in Tabelle 5.8 die Ergebnisse für das Produkt $\Sigma \cdot(\Delta V)^{2}$ aus diesem und den vorigen Kapiteln für die verschiedenen Fälle der Molekülart und -umgebung zusammengetragen und durch die Anzahl $n_{\text {exo }}$ der exozyklischen Gruppen pro Molekül in der jeweiligen Lösung geteilt. Weiterhin sind das minimale Reaktionsvolumen $\Delta V^{\text {min }}$ (berechnet mit $\Sigma^{\max }=0.25$, siehe Kapitel 1.1) pro exozyklischer Gruppe aufgelistet und der stöchiometrische Wert $\Sigma^{*}$ unter der Annahme, dass das Reaktionsvolumen pro exozyklischer Gruppe für alle Substanzen gleich dem von Glukose ist.

\begin{tabular}{l|r|r|r|r}
\hline \hline \multicolumn{1}{c|}{ Molekül } & $\begin{array}{c}\Sigma \cdot(\Delta V)^{2} \\
{\left[10^{-7} \mathrm{~L}^{2} / \mathrm{mol}^{2}\right]}\end{array}$ & $\begin{array}{c}\Sigma \cdot(\Delta V)^{2} / n_{\text {exo }} \\
{\left[10^{-7} \mathrm{~L}^{2} / \mathrm{mol}^{2}\right]}\end{array}$ & $\begin{array}{c}\Delta V^{\min } / n_{\text {exo }} \\
{[\mathrm{mL} / \mathrm{mol}]}\end{array}$ & $\begin{array}{c}\Sigma^{*} \\
\left(\Delta V / n_{\text {exo }}=1.6 \mathrm{~mL} / \mathrm{mol}\right)\end{array}$ \\
\hline \hline Glukose & 6.2 & 6.2 & 1.6 & 0.25 \\
\hline Maltose & 7.4 & 3.7 & 1.2 & 0.1 \\
\hline$\alpha$-Cyclodextrin & 13.1 & 2.2 & 0.9 & 0.1 \\
\hline $\mathrm{C}_{\mathrm{i} \mathrm{G}_{1}, \text { monomer }}$ & $\sim 6$ & $\sim 6$ & 1.5 & 0.2 \\
\hline $\mathrm{C}_{\mathrm{i}} \mathrm{G}_{1}$, mizellar & 16 & 16 & 2.5 & $0.6^{+}$ \\
\hline $\mathrm{C}_{\mathrm{i}}, \mathrm{G}_{2}$, mizellar & $\sim 50$ & $\sim 50$ & 4.5 & $2^{+}$ \\
\hline$\alpha-\mathrm{CD}+\mathrm{C}_{8} \mathrm{G}_{1}, c_{\mathrm{C} 8 \mathrm{Gl}}<c m c^{*}$ & 20 & 2.5 & 1 & 0.1 \\
\hline \hline
\end{tabular}

Tabelle 5.8: Auflistung einiger aus den Amplituden $\boldsymbol{A}_{\omega}$ abgeschätzter Größen. ${ }^{*}$ Der Wert für $\mathrm{C}_{\mathrm{i}} \mathrm{G}_{2}$ bezieht sich auf den inneren Glukosering. ${ }^{+}$Werte von $\Sigma>0.25$ sind nicht möglich - das zeigt, dass die Annahme eines nicht von der Mizellbildung abhängigen Reaktionsvolumens nicht gerechtfertigt ist.

Obwohl die Werte mit einer relativ großen Unsicherheit behaftet sind, lässt sich aus den berechneten Werten für $\Delta V^{\min } / n_{\text {exo }}$ und $\Sigma^{*}$ ablesen, dass offenbar bei Alkylglykosiden durch die Anwesenheit von Mizellnachbarn vor allem das Reaktionsvolumen der exozyklischen Rotation zunimmt. Vor diesem Hintergrund erscheint es unwahrscheinlich, dass die Abnahme der Größe $\Sigma \cdot(\Delta V)^{2} / n_{\text {exo }}$ bei Maltose, $\alpha$-CD und $\alpha-C D+\mathrm{C}_{8} \mathrm{G}_{1}$ gegenüber dem Wert von Glukose aus einer Abnahme des Reaktionsvolumens resultiert, sondern vermutlich sind diese Resultate eher mit einer ungleichmäßigeren Verteilung der Rotamerpopulationen durch die intramolekularen sterischen Wechselwirkungen und der daraus resultierenden Abnahme von $\Sigma$ zu erklären. Dies offenbart zugleich zwei grundverschiedene Arten der Beeinflussung der Konformationskinetik durch Nachbarmoleküle: Während eine Konformationsänderung eines Moleküls innerhalb einer Mizelle offenbar nur erfolgen kann, indem das Nachbarmolekül ausweichen muss, was mit einem größeren Reaktionsvolumen einhergeht, kann dies mit den vergleichsweise großen und starren Cyclodextrin-Molekülen nicht geschehen - dementsprechend ist in diesem Fall die Konformationskinetik stark eingeschränkt. 


\section{Zusammenfassung und Ausblick}

In der vorliegenden Arbeit werden insgesamt 74 Ultraschallabsorptionsspektren diskutiert, die über einen Frequenzbereich von ca. $100 \mathrm{kHz}$ bis $400 \mathrm{MHz}$ aufgenommen worden waren. Darunter sind 56 Spektren von Lösungen 10 verschiedener Alkylglykoside mit Alkylkettenlängen von $n_{c}=6$ bis $n_{c}=10$, weiterhin 5 Spektren von Maltose- und Glukose-Lösungen zur Erweiterung von Literaturdaten zu niedrigeren Konzentrationen, 11 Spektren für Lösungen von Mischungen eines Alkylglykosids $\left(\mathrm{C}_{8} \mathrm{G}_{1}\right)$ mit $\alpha$-Cyclodextrin und 2 Spektren für Lösungen von Mischungen zweier verschiedener Alkylglykoside $\left(\mathrm{C}_{8} \mathrm{G}_{1}+\mathrm{C}_{8} \mathrm{Gal}\right)$. Die einzelnen Spektren können im Rahmen der Fehler zufriedenstellend als Überlagerung aus bis zu vier Debye-Spektralfunktionen beschrieben werden. Für diese Debye-Spektralfunktionen können ein oder zwei Monomeraustauschprozesse, eine (teilweise) Rotation der glykosidischen Bindung, eine (teilweise) Rotation der exozyklischen Gruppe und im Falle der $\alpha$ $\mathrm{CD} / \mathrm{C}_{8} \mathrm{G}_{1}$-Mischungen eine Komplexbildungsreaktion als molekularkinetische Ursache identifiziert werden. Aus der Betrachtung der Konzentrationsabhängigkeit (und in einem Fall der Temperaturabhängigkeit) der Parameter Relaxationszeit $\tau$ und Relaxationsamplitude $A$ werden Rückschlüsse auf die molekulare Kinetik der einzelnen Prozesse, ihre Stöchiometrie, ihre Beeinflussung durch sterische Wechselwirkungen mit Nachbarmolekülen sowie auf die Mizellgeometrie gezogen.

Das Auftreten zweier Monomeraustauschprozesse bei einigen Alkylglykosiden kann mit der Existenz zweier Mizellformen oder mit einer stark von der Kugelform abweichenden Mizellgeometrie erklärt werden. Für das Alkylglykosid $\mathrm{C}_{8} \mathrm{G}_{1}$ wird eine Konzentration von etwa $75 \mathrm{mmol} / \mathrm{L}$ als Umwandlungskonzentration ausgemacht. Die Rückreaktionskonstanten $k_{b}$ des Monomeraustauschprozesses weisen unabhängig davon, ob ein zweiter Prozess auftritt, eine auch für andere Tensidklassen gültige universelle Abhängigkeit von der kritischen Mizellkonzentration $c m c$ auf, während die Reaktionsvolumina bei einigen Alkylglykosiden zwar einen universellen Wert von etwa $1.5 \mathrm{~mL} / \mathrm{mol}$ pro $\mathrm{CH}_{2}$-Gruppe der Alkylkette aufweisen, aber diese Werte in anderen Fällen durch das Auftreten eines zweiten Monomeraustauschprozesses deutlich geringer ausfallen. Dieser Befund beruht vermutlich darauf, dass die Theorien, die zur Auswertung benutzt wurden, nur für kugelförmige Mizellen gelten. In diesem Zusammenhang wären zum einen genauere Kenntnisse der Geometrie von Alkylglykosid-Mizellen in Abhängigkeit von der Konzentration und zum anderen Ausweitungen der Theorien der Mizellkinetik auf nicht kugelförmige Mizellen wünschenswert.

Eine konkrete quantitative Auswertung der Parameter des Prozesses der teilweisen Rotation der glykosidischen Bindung gestaltet sich als schwierig, da über die einzelnen Rotationszustände wenig bekannt ist und daher nicht ausgeschlossen werden kann, dass es sich bei diesem Prozess um eine Überlagerung mehrerer Prozesse mit gleicher oder ähnlicher Relaxationszeit handelt. Dennoch ergeben sich einige qualitative Erkenntnisse aus den Ergebnissen: Die Reaktionskinetik ist im Falle einer Glukose-Glukose-Bindung etwas schneller als bei GlukoseAlkyl-Bindungen. Weiterhin ist für beide Bindungstypen die skalierte Prozessamplitude im mizellaren Zustand deutlich größer als im monomeren Zustand - vermutlich hauptsächlich dadurch bedingt, dass das Reaktionsvolumen des Prozesses in einer Mizelle bis zu 5mal größer ist als außerhalb. Für die Alkylmaltoside, die sowohl eine Bindung Glukose-Glukose als auch eine Bindung Glukose-Alkyl aufweisen, zeigt sich, dass erstere deutlich stärker zur Ultraschallabsorption beiträgt. Dies kann damit begründet werden, dass die Glukose-Alkyl-Bindung näher am Mizellkern vorliegt, wo die Beeinflussung durch Nachbarmoleküle stärker ausfällt. Für weitere Er- 
kenntnisse über diesen molekularen Prozess wären Computer-Simulationen von Vorteil, die die Konformationskinetik von Disacchariden oder Alkylmaltosiden über einen Zeitraum von mindestens einer Mikrosekunde simulieren. Die aus solchen Simulationen resultierenden Erkenntnisse über die Anzahl der möglichen Zustände und ihrer Populationen wären dann für eine quantitative Analyse der Messdaten nützlich.

Für die Rotation der exozyklischen Gruppe lässt sich zeigen, dass das Reaktionsvolumen dieses Prozesses bei Alkylglykosiden im monomeren Zustand etwa dem von Glukose und Maltose entspricht, während es im mizellaren Zustand mindestens 1.5mal so groß ist. Für Alkylmaltoside im mizellaren Zustand ergibt eine Abschätzung, dass das Reaktionsvolumen des Glukoseringes, der sich näher am Mizellkern befindet, sogar mindestens 2 bis $4 \mathrm{mal}$ so groß ist. Auch diese Erkenntnisse lassen sich damit erklären, dass die Wechselwirkung mit Nachbarmolekülen innerhalb einer Mizelle umso stärker ist, je näher am Mizellkern die betrachtete Reaktion stattfindet.

Die Messungen an $\alpha$-Cyclodextrin $/ \mathrm{C}_{8} \mathrm{G}_{1}$-Mischungen bestätigen das in der Literatur bevorzugte Modell, dass bei kleinen Tensidkonzentrationen die Tensidmoleküle bevorzugt mit den Cyclodextrin-Molekülen komplexieren, bis bei einer bestimmten Konzentration, der scheinbaren $c m c^{*}$, ein sehr großer Teil der Cyclodextrin-Moleküle ,belegt' ist. Oberhalb dieser $\mathrm{cmc}$ * erweist sich die Kinetik und die Stöchiometrie des Monomeraustauschprozesses als kaum oder gar nicht verändert gegenüber reinen Tensid-Lösungen. Weiterhin zeigt sich in den Ultraschallabsorptionsspektren der $\alpha-\mathrm{CD} / \mathrm{C}_{8} \mathrm{G}_{1}$-Mischungen ein Prozess, der der Komplexbildung der beiden Spezies zugeordnet werden muss. Der Verlauf der relevanten Relaxationsamplituden über der Tensidkonzentration lässt sich dabei nur mit der Existenz von $\mathrm{T}_{2}{ }^{\circ} \mathrm{CD}$-Komplexen erklären - in der Literatur wird die Existenz dieser Komplexe allerdings bestritten. Möglicherweise könnten weitere Messungen an Mischungen mit unterschiedlicher Cyclodextrin-Konzentration hier weitere Erkenntnisse hervorbringen. Die Rotation der glykosidischen Bindung und der exozyklischen Gruppen lässt sich in den $\alpha-C D / C_{8} G_{1}$-Mischungen insoweit analysieren, als für beide molekularen Prozesse eine deutliche Beeinflussung der Konformationskinetik nachweisbar ist, die in beiden Fällen vor allem durch sterische Wechselwirkungen mit dem jeweiligen Komplexpartner erklärt werden können.

Eine der beiden Hauptintentionen dieser Arbeit war die Beantwortung der Frage nach qualitativen und quantitativen Kenntnissen über grundlegende inter- und intramolekulare Wechselwirkungen der für Wissenschaft und Industrie interessanten Substanzklasse der Alkylglykoside. Die entsprechenden Untersuchungen brachten qualitativ erklärbare und quantitativ sinnvoll erscheinende Ergebnisse hervor, die möglicherweise eine Grundlage für weitere Messungen an dieser Substanzklasse bilden und bei der Beantwortung noch offener Fragestellungen hilfreich seien können.

Die Frage nach der Beeinflussung der Konformationskinetik bekannter molekularer Prozesse durch sterische Wechselwirkungen mit Nachbarmolekülen, also die zweite Hauptintention dieser Arbeit, konnte qualitativ sowohl für intramizellare Wechselwirkungen als auch für Wechselwirkungen zwischen den Komplexbildungspartnern $\alpha-C D$ und $\mathrm{C}_{8} \mathrm{G}_{1}$ nachgewiesen und erklärt werden. Ein entscheidendes Ergebnis ist hierbei, dass die Konformationskinetik sowohl im Falle von Mizellen eine größere Prozessamplitude aufweist als bei freien Molekülen, als auch bei den $\alpha-C D-C_{8} G_{1}$-Komplexen gegenüber den unkomplexierten Molekülen - vermutlich ist dieses Verhalten dadurch bedingt, dass innerhalb der Mizellen bzw. innerhalb der Komplexe eine Konformationsänderung unweigerlich mit einem Ausweichen der Nachbarmoleküle (und damit mit einem entsprechend größeren Reaktionsvolumen) verbunden ist. Allerdings sind für genauere Aussagen zu dieser Thematik noch zahlreiche weitere Messungen vonnöten. 


\section{$7 \quad$ Literaturverzeichnis}

[1] Gelbart, W. M.; Ben-Shaul, A.; Roux, D. (Eds.), Micelles, Membranes, Microemulsions, and Monolayers (Partially Ordered Systems), Springer Verlag New York (1994)

[2] Skript zum Kurs 'Biological Sciences', University of Illinois at Chicago (2004)

[3] Lange, K. R., Industrial Applications of Surfactants in Lange, K. R. (Ed.), Surfactants: A Practical Handbook, Hanser Gardner Publications, 144-170 (1999)

[4] Karlson, P., Doenecke, D., Koolman, J., Kurzes Lehrbuch der Biochemie für Mediziner und Naturwissenschaftler, Georg Thieme Verlag Stuttgart, 14. Auflage (1994)

[5] Henry, G. D.; Sykes, B. D., Methods to Study Membrane Protein Structure in Solution, Meth. Enzymol., $239,515-535$ (1994)

[6] Hubbell, J. A., Enhancing Drug Function, Science, 300, 595-596 (2003)

[7] Lawrence, M. J., Surfactant Systems: Their Use in Drug Delivery, Chem. Soc. Rev., 23, 417-424 (1994)

[8] Aniansson, E. A. G.; Wall, S. N., On the Kinetics of Step-Wise Micelle Association, J. Phys. Chem., 76(10), 1024-1030 (1974)

[9] Aniansson, E. A. G., Dynamics and Structure of Micelles and other Amphiphilic Structures, J. Phys. Chem., 82(26), 2805-2808 (1978)

[10] Aniansson, E. A. G., Theory of Micelle Formation Kinetics, Ber. Bunsenges. Phys. Chem., 82, 981-988 (1978)

[11] Aniansson, E. A. G., The Mean Lifetime of a Micelle, Progr. Colloid \& Polymer Sci., 70, 2-5 (1985)

[12] Aniansson, E. A. G.; Wall, S. N.; Almgren, M.; Hoffmann, H.; Kielmann, I.; Ulbricht, W.; Zana, R.; Lang, J.; Tondre, C., Theory of Kinetics of Micellar Equilibria and Quantitative Interpretation of Chemical Relaxation Studies of Micellar Solutions of Ionic Surfactants, J. Phys. Chem., 80, 905-922 (1976)

[13] Wall, S. N.; Aniansson, E. A. G., Numerical Calculations on the Kinetics of Stepwise Micelle Association, J. Phys. Chem., 84, 727-736 (1980)

[14] Kahlweit, M., What do we know about Micelles and which Questions are still open?, Pure \& Appl. Chem., 53, 2069-2081 (1981)

[15] Balzer, D.; Lüders, H. (Eds.), Nonionic Surfactants: Alkyl Polyglucosides, Surfactant Science Series 91, Marcel Dekker Ltd (2000)

[16] Koshland, D. E., Application of a Theory of Enzyme Specificity to Protein Synthesis, Proc. Nat. Acad. Sci., 44(2), 98-104 (1958) 
[17] Kaatze, U.; Hushcha, T. O.; Eggers, F., Ultrasonic Broadband Spectrometry of Liquids: A Research Tool in Pure and Applied Chemistry and Chemical Physics, J. Sol. Chem., 29(4), 299-368 (2000)

[18] Neubig, B.; Briese, W., Das große Quarzkochbuch, Franzis Verlag Feldkirchen (1997)

[19] Bhatia, A. B., Ultrasonic Absorption, Oxford University Press (1967)

[20] Strehlow, H., Rapid Reactions in Solution, VCH Verlagsgesellschaft Weinheim (1992)

[21] Vogel, H., Gerthsen Physik, Springer Verlag Berlin, 18. Auflage (1995)

[22] International Association for the Properties of Water and Steam, Release on the IAPWS Formulation 1995 for the Thermodynamic Properties of Ordinary Water Substance for General and Scientific Use, Executive Secretary R. B. Dooley, Electric Power Research Inst., Pablo Alto (1995)

[23] Menzel, K.; Ultraschallspektroskopie an $C_{i} E_{j}$ /Wasser-Mischungen als Funktion der Zusammensetzung und Temperatur, Dissertation, Mat.-Nat. Fak. Univ. Göttingen (1993)

[24] Kaatze, U., Lautscham, K.; Below Cut-Off Piston Attenuator as a Calculable Signal Vernier for Microwaves up to 15 GHz., J. Phys. E: Sci. Instrum., 19, 1046 (1986)

[25] Kaatze, U.; Kühnel, V.; Menzel, K.; Schwerdtfeger, S., Ultrasonic Spectroscopy of Liquids. Extending the Frequency Range of the Variable Sample Length Pulse Technique, Meas. Sci. Technol., 4, 1257-1265 (1993)

[26] Kaatze, U.; Lautscham, K.; Brai, M., Acoustical Absorption Spectroscopy of Liquids Between 0.15 and 3000 MHz: II. Ultrasonic Pulse Transmission Methods, J. Phys. E: Sci. Instrum., 21, 98-103 (1988)

[27] Labhardt, A., Konstruktion und Berechnung von Zylinderresonatoren zur Schallabsorptions- und Dispersionsmessung chemischer Relaxationsprozesse, Dissertation, Philosophisch-Naturwissenschaftliche Fakultät, Universität Basel (1975)

[28] Meyer, E.; Neumann, E. G., Physikalische und Technische Akustik, Vieweg Verlag Braunschweig (1979)

[29] Polacek, R., Breitbandige Ultraschallabsorptionsspektroskopie an wässrigen ionischen Tensid-Lösungen im Frequenzbereich von $100 \mathrm{kHz}$ bis $2 \mathrm{GHz}$, Dissertation, Mathematisch-naturwissenschaftliche Fakultäten, Georg-August-Universität Göttingen (2003)

[30] Von Rybinski, W.; Hill, K., Alkyl Polyglycosides - Properties and Applications of a new Class of Surfactants, Angew. Chem. Int. Ed., 37, 1328-1345 (1998)

[31] Christen, H. R., Grundlagen der organischen Chemie, Verlag Sauerländer Aarau Diesterweg, 1. Auflage (1970)

[32] Mortimer, C. E., Chemie, Georg Thieme Verlag Stuttgart, 5. Auflage (1987)

[33] Lehmann, J., Kohlenhydrate, Georg Thieme Verlag Stuttgart, 2. Auflage (1996) 
[34] Polacek, R.; Stenger, J.; Kaatze, U., Chair-Chair Conformational Flexibility, Pseudorotation, and Exocyclic Group Isomerization of Monosaccharides in Water, J. Chem. Phys., 116(7), 2973-2982 (2002)

[35] Brant, D. A., Conformation and Behavior of Polysaccharides in Solution. In Preiss, J. (Ed.), The Biochemistry of Plants, Vol. 3, Carbohydrates: Structure and Function, Academic Press London, 425-472 (1980)

[36] Ott, K.-H.; Meyer, B., Molecular Dynamics Simulations of Maltose in Water, Carbohydr. Res., 281, 11-34 (1996)

[37] Kuttel, M. M.; Naidoo, K. J., Free Energy Surfaces for the $\alpha(1 \rightarrow 4)$-Glycosidic Linkage: Implications for Polysaccharide Solution Structure and Dynamics, J. Phys. Chem. B, 109, 7468-7474 (2005)

[38] Hagen, R.; Breitbandige Ultraschallabsorptionsspektroskopie an wässrigen Kohlenhydrat-Lösungen, Dissertation, Mathematisch-naturwissenschaftliche Fakultäten, Georg-August-Universität Göttingen (2003)

[39] Frank, M., Konformationsanalyse von Oligosacchariden im freien und gebundenen Zustand, Dissertation, Naturwissenschaftlich-Mathematische Gesamtfakultät, Universität Heidelberg (2000)

[40] Cheetham, N. W. H.; Dasgupta, P.; Ball, G. E., NMR and Modelling Studies of Disaccharide Conformation, Carb. Res., 338, 955-962 (2003)

[41] Eklund, R., Widmalm, G., Molecular Dynamics Simulations of an Oligosaccharide using a Force Field Modified for Carbohydrates, Carbohydr. Res., 338, 393-398 (2003)

[42] Pereira, C. S.; Kony, D.; Baron, R.; Müller, M.; van Gunsteren, W. F.; Hünenberger, P. H., Conformational and Dynamical Properties of Disaccharides in Water: a Molecular Dynamics Study, Biophys. J., 90, 4337-4344 (2006)

[43] Kirschner, K. N.; Woods, R. J., Solvent Interactions Determine Carbohydrate Conformation, Proc. Nat. Acad. Sci., 98(19), 10541-10545 (2001)

[44] Mason, P. E.; Weilson, G. W.; Enderby, J. E.; Saboungi, M.-L.; Cuello, G.; Brady, J. W., Neutron Diffraction and Simulation Studies of the Exocyclic Hydroxymethyl Conformation of Glucose, J. Chem. Phys., 125,224505 (2006)

[45] Behrends, B.; Cowman, M. K.; Eggers, F.; Eyring, E. M.; Kaatze, U.; Majewski, J.; Petrucci, S.; Richmann, K.-H.; Riech, M., Ultrasonic Relaxation and Fast Chemical Kinetics of Some Carbohydrate Aqueous Solutions, J. Am. Chem. Soc., 119, 2182-2186 (1997)

[46] Behrends, R.; Kaatze, U., Molecular Dynamics and Conformational Kinetics of Mono- and Disaccharides in Aqueous Solution, ChemPhysChem, 6(6), 1133-1145 (2005)

[47] Hagen, R.; Kaatze, U., Conformational Kinetics of Disaccharides in Aqueous Solutions, J. Chem. Phys., 120(20), 9656-9664 (2004) 
[48] Polacek, R.; Kaatze, U., Monomer Exchange Kinetics, Radial Diffusion, and Hydrocarbon Chain Isomerization of Sodium Dodecylsulfate Micelles in Water, J. Phys. Chem. B, 111(7), 1625-1631 (2007)

[49] Haller, J.; Miecznik, P.; Kaatze, U., Ultrasonic Attenuation Spectrometry Study of $\alpha$-Cyclodextrin + KI Complexation in Water, Chem. Phys. Lett., 429, 97-102 (2006)

[50] Nishida, Y.; Ohrui, H.; Meguro, H., ${ }^{1}$ H-NMR Studies of (6R)- and (6S)-Deuterated D-Hexoses: Assignment of the Preferred Rotamers about C5-C6 Bond of D-Glucose and D-Galactose Derivatives in Solutions, Tetrahed. Lett., 25(15), 1575-1578 (1984)

[51] Bock, K.; Duus, J. Ø., A Conformational Study of Hydroxymethyl Groups in Carbohydrates Investigated by ${ }^{I}$ H NMR Spectroscopy, J. Carb. Chem., 13(4), 513-543 (1994)

[52] Kohn, W., Nobel Lecture:Electronic Structure of Matter - Wave Functions and Density Functionals, Revs. Mod. Phys.,71(5), 1253-1266 (1999)

[53] Schnupf, U.; Willett, J. L.; Bosma, W. B.; Momany, F. A., DFT Studies of the Disaccharide, $\alpha$-Maltose: Relaxed Isopotential Maps, Carb. Res., 342, 2270-2285 (2007)

[54] Chan, S. K., Herrmann, U.; Ostner, W.; Kahlweit, M., Kinetics of Formation of Ionic Micelles. 1. Analysis of Amplitudes, Ber. Bunsenges. Phys. Chem., 81(1), 60-66 (1977)

[55] Chan, S. K., Herrmann, U.; Ostner, W.; Kahlweit, M., Kinetics of Formation of Ionic Micelles. 2. Analysis of Time Constants, Ber. Bunsenges. Phys. Chem., 81(4), 396-402 (1977)

[56] Bagchi, B., Water Dynamics in the Hydration Layer around Proteins and Micelles, Chem. Rev., 105(9), 3197-3219 (2005)

[57] Huibers, P. D. T.; Lobanov, V. S.; Katritzky, A. R.; Shah, D. O.; Karelson, M., Prediction of Critical Micelle Concentration Using a Quantitative Structure-Property Relationship Approach. 1. Nonionic Surfactants, Langmuir, 12, 1462-1470 (1996)

[58] Yuan, S. L.; Cai, Z. T. ; Xu, G. Y. ; Jiang, Y. S., Quantitative Structure-Property Relationships of Surfactants: Prediction of the Critical Micelle Concentration of Nonionic Surfactants, Coll. \& Pol. Sci., 280(7), 630-636 (2002)

[59] Wang, Z. W.; Huang, D. Y.; Gong, S. P.; Li, G. Z., Prediction on Critical Micelle Concentration of Nonionic Surfactants in Aqueous Solution: Quantitative Structure-Property Relationship Approach, Chin. J. Chem., 21(12), 1573-1579 (2003)

[60] Patist, A.; Jha, B. K.; Oh, S. G.; Shah, D. O., Importance of Micellar Relaxation Time on Detergent Properties, J. Surf. Det., 2(3), 317-324 (1999)

[61] Danov, K. D.; Kralchevsky, P. A.; Denkov, N. D.; Ananthapadmanabhan, K. P.; Lips, A., Mass Transport in Micellar Surfactant Solutions: 1. Relaxation of Micelle Concentration, Aggregation Number and Polydispersity, Adv. Coll. Interf. Sci., 119, 1-16 (2006) 
[62] Kaatze, U.; Behrends, R., Liquids: Formation of Complexes and Complex Dynamics in: Kurz, T.; Parlitz, U.; Kaatze, U. (Eds.), Oscillations, Waves, and Interactions: Sixty Years Drittes Physikalisches Institut, Festschrift, Universitätsverlag Göttingen (2007)

[63] Telgmann, T; Kaatze, U., On the Kinectics of the Formation of Small Micelles. 1. Broadband Ultrasonic Spectrometry, J. Phys. Chem. B, 101, 7758-7765 (1997)

[64] Telgmann, T; Kaatze, U., On the Kinectics of the Formation of Small Micelles. 2. Extension of the Model of Stepwise Association, J. Phys. Chem. B, 101, 7766-7772 (1997)

[65] Trachimov, C., Messung extrem langer Relaxationszeiten von wässrigen Mizell-Lösungen und Simulation der Relaxationsprozesse aufgrund eines theoretischen Modells, Dissertation, Mathematischnaturwissenschaftliche Fakultäten, Georg-August-Universität Göttingen (1996)

[66] Teubner, M., Theory of Ultrasonic Absorption in Micellar Solutions, J. Phys. Chem., 83(22), 2917-2920 (1979)

[67] Tanford, C., Micelle Shape and Size, J. Phys. Chem., 76(21), 3020-3024 (1972)

[68] Telgmann, T., Akustische Spektroskopie an wäßrigen Mischungen nichtionischer Tenside des Typs $C_{i} E_{j}$ mit Mischungslücke, Dissertation, Mathematisch-naturwissenschaftliche Fakultäten, Georg-AugustUniversität Göttingen (1997)

[69] Patist, A.; Kanicky, J. R.; Shukla, P. K.; Shah, D. O., Importance of Micellar Kinetics in Relation to Technological Processes, J. Coll. Interf. Sci., 245, 1-15 (2002)

[70] Israelachvili, J. N., Thermodynamic and Geometric Aspects of Amphiphile Aggregation into Micelles, Vesicles and Bilayers, and the Interactions between them in: Degiorgio, V.; Corti, M., Physics of Amphiphiles: Micelles, Vesicles and Microemulsions - Proceedings of the International School of Physics «Enrico Fermi» Course XC, North Holland, 24-58 (1995)

[71] Israelachvili, J. N.; Mitchell, D. J.; Ninham, B. W., Theory of Self-Assembly of Hydrocarbon Amphiphiles into Micelles and Bilayers, J. Chem. Soc. Far. Trans. II, 72, 1525-1568 (1976)

[72] Tanford, C., Theory of Micelle Formation in Aqueous Solutions, J. Phys. Chem., 78(24), 2469-2479 (1974)

[73] Almgren, M.; Alsins, J.; van Stam, J.; Mukhtar, E., Prog. Coll. Polym. Sci., 76, 68 (1988)

[74] Kuni, F. M.; Shchekin, A. K.; Grinin, A. P.; Rusanov, A. I., Kinetic Description of the Relaxation of Surfactant Solutions Containing Spherical and Cylindrical Micelles, Coll. J., 67(1), 41-50 (2005)

[75] Kuni, F. M.; Shchekin, A. K.; Rusanov, A. I; Grinin, A. P., Concentrations of Monomers and Cylindrical Micelles above the Second CMC, Coll. J., 66(2), 174-185 (2004)

[76] Roxby, R. W.; Mills, B. P., Micelle Size Distribution and Free Monomer Concentration in Aqueous Solutions of Octyl Glucoside, J. Phys. Chem., 94, 456-459 (1990) 
[77] Brown, G. M.; Dubreuil, P.; Ichhapor, F. M.; Desnoyer, J. E., Synthesis and Properties of some Alpha-Dalkyl Glucosides and Mannosides - Apparent Molal Volumes and Solubilization of Nitrobenzene in Water at $25^{\circ} \mathrm{C}$, Can. J. Chem., 48(16), 2525-2531 (1970)

[78] Nelson, P. H., Rutledge, G. C.; Hatton, T. A., On the Size and Shape of Self-Assembled Micelles, J. Chem. Phys., 107(24),10777-10781 (1997)

[79] Zhang, R.; Marone, P. A.; Thiyagarajan, P.; Tiede, D. M., Structure and Molecular Fluctuations of nAlkyl- $\beta$-D-Glucopyranoside Micelles Determined by X-ray and Neutron Scattering, Langmuir, 15, 75107519 (1999)

[80] Aoudia, M.; Zana, R., Aggregation Behavior of Sugar Surfactants in Aqueous Solutions: Effects of Temperature and the Addition of Nonionic Polymers, J. Coll. Interf. Sci., 206, 158-167 (1998)

[81] Pastor, O.; Junquera, E.; Aicart, E., Hydration and Micellization Processes of n-Octyl $\beta$-DGlucopyranoside in Aqueous Solution. A Thermodynamic and Fluorimetric Study in the Absence and Presence of Salts, Langmuir, 14, 2950-2957 (1998)

[82] Giordano, R.; Maisano, G.; Teixeira, J., SANS Studies of Octyl- $\beta$-Glucoside and Glycine Micellar Solutions, J. Appl. Cryst., 30, 761-764 (1997)

[83] He, L., Self-Assembly and Fundamental Aspects of Affinity Adsorption of Glycoconjugates, Dissertation, Fachbereich Maschinenbau, Universität der Bundeswehr Hamburg (2002)

[84] Frindi, M.; Michels, B.; Zana, R., Ultrasonic Absorption Studies of Surfactant Exchange between Micelles and Bulk Phase in Aqueous Micellar Solutions of Nonionic Surfactants with a Short Alkyl Chain. 3. Surfactants with a Sugar Head Group, J. Phys. Chem., 96, 8137-8141 (1992)

[85] Lässer, H. R.; Elias, H. G., Zur Assoziation von Seifen. III. Das Assoziationsverhalten von $\beta$-D-nOctylGlukosid in Wasser, Kolloid-Z. u. Z. Polymere, 250, 58-63 (1972)

[86] Le Maire, M.; Champeil, P.; Møller, J. V., Interaction of Membrane Proteins and Lipids with Solubilizing Detergents, Biochim. Biophys. Acta, 1508, 86-111 (2000)

[87] Lorber, B.; Bishop, J. B.; DeLucas, L. J., Purification of Octyl $\beta$-D-Glucopyranoside and Re-estimation of its Micellar Size, Biochim. Biophys. Acta, 1023, 254-265 (1990)

[88] Bucci, S.; Fagotti, C.; Degiorgio, V.; Piazza, R., Small-Angle Neutron-Scattering of Ionic-Nonionic Mixed Micelles, Langmuir, 7, 824-826 (1991)

[89] Zhang, R.; Somasundaran, P., Abnormal Micellar Growth in Sugar-Based and Ethoxylated Nonionic Surfactants and Their Mixtures in Dilute Regimes Using Analytical Ultracentrifugation, Langmuir, 20(20), 8552-8558 (2004)

[90] Dupuy, C.; Auvray, X.; Petipas, C.; Rico-Lattes, I. ; Lattes, A., Anomeric Effects on the Structure of Micelles of Alkyl Maltosides in Water, Langmuir, 13, 3965-3967 (1997) 
[91] Warr, G. G.; Drummond, C. J.; Grieser, F.; Ninham, B. W.; Evans, D. F., Aqueous Solution Properties of Nonionic n-Dodecyl $\beta$-D-Maltoside Micelles, J. Phys. Chem., 90, 4581-4586 (1986)

[92] Lu, S.; Somasundaran, P., Coexistence and Growth of Micellar Species in a Sugar-Based Surfactant/Phenol Mixture Studied by Analytical Ultracentrifugation, Langmuir, 23, 9188-9194 (2007)

[93] Gröger, M.; Kretzer, E. K.; Woyke, A., Reader mit Hintergrundinformationen zum Thema Cyclodextrine, Science-Forum, Universität Siegen (2001) erhältlich via www.science-forum.de/download/cyclodex.pdf

[94] Kato, S.; Nomura, H.; Miyahara, Y., Ultrasonic Relaxation Study of Aqueous Solutions of Cyclodextrins, J. Phys. Chem., 89, 5417-5421 (1985)

[95] Fukahori, T.; Kondo, M.; Nishikawa, S., Dynamic Study of Interaction between $\beta$-Cyclodextrin and Aspirin by the Ultrasonic Relaxation Method, J. Phys. Chem. B, 110(9), 4487-4491 (2006)

[96] Nishikawa, S.; Yokoo, N.; Kuramoto, N., Kinetic Study for Complexation between a-Cyclodextrin and Alcohols in Water by the Ultrasonic Relaxation Method, J. Phys. Chem. B, 102, 4830-4834 (1998)

[97] Nishikawa, S.; Kondo, M., Kinetic Study for the Inclusion Complex of Carboxylic Acids with Cyclodextrin by the Ultrasonic Relaxation Method, J. Phys. Chem. B, 110, 26143-26147 (2006)

[98] Cunha-Silva, L.; Teixeira-Dias, J. J. C., Aqueous Solution Inclusion of the Nonionic Surfactant C12E4 in $\beta$-Cyclodextrin: Implications of Micellization in Stoichiometry Determination and Model Calculations, J. Incl. Phen. Macrocyc. Chem., 43, 127-131 (2002)

[99] Junquera, E.; Tardajos, G.; Aicart, E., Effect of the Presence of $\beta$-Cyclodextrin on the Micellization Process of Sodium Dodecyl Sulfate or Sodium Perfluorooctanoate in Water, Langmuir, 9, 1213-1219 (1993)

[100] Reinsborough, V. C.; Stephenson, V. C., Inclusion Complexation Involving Sugar-Containing Species: $\beta$ Cyclodextrin and Sugar Surfactants, Can. J. Chem., 81(1), 45-49 (2004)

[101] Valente, A. J. M.; Nilsson, M.; Söderman, O., Interactions between n-Octyl and n-Nonyl $\beta$-D-Glucosides and $\alpha$ - and $\beta$-Cyclodextrins as Seen by Self-Diffusion NMR, J. Coll. Interf. Sci., 281, 218-224 (2005)

[102] Cabaleiro-Lago, C.; García-Río, L.; Hervés, P.; Mejuto, J. C.; Pérez-Juste, J., In Search of Fully Uncomplexed Cyclodextrin in the Presence of Micellar Aggregates, J. Phys. Chem. B, 110, 15831-15838 (2006)

[103] Dorrego, B.; García-Río, L.; Hervés, P.; Leis, J. R.; Mejuto, J. C.; Pérez-Juste, J., Changes in the Fraction of Uncomplexed Cyclodextrin in Equilibrium with the Micellar System as a Result of Balance between Micellization and Cyclodextrin-Surfactant Complexation. Cationic Alkylammonium Surfactants, J. Phys. Chem. B, 105, 4912-4920 (2001)

[104] Anatrace-Produktkatalog, $2^{\text {nd }}$ Edition (2004)

[105] Eriksson, J.C.; Ljunggren, S., The Mechanics and Thermodynamics of Rod-shaped Micelles, J. Chem. Soc., Farad. Trans. 2, 81, 1209-1242 (1985) 
[106] Ljunggren, S.; Eriksson, J. C., The Mechanics and Thermodynamics of Disc-shaped Micelles, J. Chem. Soc., Farad. Trans. 2, 82, 913-928 (1986)

[107] Bogusz, S; Venable, R. M.; Pastor, R.W.; Molecular Dynamics Simulations of Octyl Glucoside Micelles: Structural Properties, J. Phys. Chem. B, 104, 5462-5470 (2000)

[108] Riech, M., Breitbandige Ultraschallspektrometrie an wäßrigen Lösungen von Monosaccariden mit und ohne Salzzusatz, Diplomarbeit, Georg-August-Universität Göttingen (1996)

[109] Hall, D. G.; Wyn-Jones, E., Chemical Relaxation Spectrometry in Aqueous Surfactant Solutions, J. Mol. Liq., 32, 1, 63-82 (1986)

[110] Nomura, H.; Koda, S.; Matsuoka, T.; Hiyama, T.; Shibata, R.; Kato, S., Study of Salt Effects on the Micelle-Monomer Exchange Process of Octyl-, Decyl-, and Dodecyltrimethylammonium Bromide in Aqueous Solutions by Means of Ultrasonic Relaxation Spectroscopy, J. Coll. Interf. Sci., 230, 1, 22-28 (2000)

[111] Frindi, M.; Michels, B.; Zana, R., Ultrasonic Absorption Studies of Surfactant Exchange between Micelles and Bulk Phase in Aqueous Micellar Solutions of Nonionic Surfactants with a Short Alkyl Chain. 2. $C_{6} E_{3}, C_{6} E_{5}, C_{8} E_{4}$, and $C_{8} E_{8}$, J. Phys. Chem., 96, 6095-6102 (1992)

[112] Kato, S.; Harada, S.; Sahara, H., Ultrasonic Relaxation and Volumetric Studies of the Micelle/Monomer Exchange Process in Aqueous Solutions of the Nonionic Surfactants $C_{7} E_{4}, C_{8} E_{4}$, and $C_{8} E_{5}$, J. Phys. Chem., 99, 12570-12575 (1995)

[113] Fukada, K.; Kawasaki, M.; Seimiya, T.; Abe, Y.; Fujiwara, M.; Ohbu, K., Stereochemical Aspects of Micellar Properties of Esterified Glucoside Surfactants in Water: Apparent Molar Volume, Adiabatic Compressibility, and Aggregation Number, Coll. Polym. Sci., 278, 576-580 (2000)

[114] Haller, J., Untersuchungen über die Wechselwirkung zwischen der Mizellkinetik und der Entmischungsdynamik in wässrigen Lösungen des nichtionischen Tensids $C_{7} E_{3}$, Diplomarbeit, Georg-August-Universität Göttingen (2004)

[115] Majhi, P. R., Blume, A., Thermodynamic Characterization of Temperature-Induced Micellization and Demicellization of Detergents Studied by Differential Scanning Calorimetry, Langmuir, 17, 3844-3851 (2001)

[116] La Mesa, C.; Bonincontro, A.; Sesta, B., Solution Properties of Octyl $\beta$-D Glucoside. Part 1: Aggregate Size, Shape and Hydration, Colloid Polym Sci, 271, 1165-1171 (1993)

[117] Piñ eiro, A., Banquy, X., Pérez-Casas, S., Tovar, E., Garcia, A., Villa, A., Amigo, A., Mark, A. E., Costas, M., On the Characterization of Host-Guest Complexes: Surface Tension, Calorimatry, and Molecular Dynamics of Cyclodextrins with a Non-ionic Surfactant, J. Phys. Chem. B, 111, 4383-4392 (2007)

[118] Mwakibete, H.; Cristantino, R.; Bloor, D. M.; Wyn-Jones, E.; Holzwarth, J. F., Reliability of the Experimental Methods To Determine Equilibrium Constants for Surfactant/Cyclodextrin Inclusion Complexes, Langmuir, 11, 57-60 (1995) 



\section{Danksagung}

Herrn Prof. Dr. Werner Lauterborn danke ich für die Betreuung und die Begutachtung dieser Arbeit.

Herrn PD Dr. C. Jooß danke ich, dass er bereit ist, das Korreferat dieser Arbeit zu übernehmen.

Herrn Dr. Udo Kaatze danke ich für stets hilfreiche Diskussionen und Vorschläge und die außerordentlich gute Atmosphäre in der Arbeitsgruppe.

Allen aktiven und ehemaligen Mitgliedern der Arbeitsgruppe „Komplexe Fluide“ danke ich für anregende Diskussionen, die Hilfe bei der Einarbeitung an den Apparaturen und die stete Bereitschaft bei auftretenden Problemen zu helfen. Insbesondere möchte ich dabei Herrn Dr. Ralph Behrends, Herrn Dr. Ralf Hagen und Herrn Dr. Irek Iwanowski dankend erwähnen.

Den Mitarbeitern der Elektronik- und der Mechanikwerkstatt des Dritten Physikalischen Institutes möchte ich meinen Dank für schnelle Reparaturen bei auftretenden Problemen aussprechen.

Meinen ehemaligen und aktuellen Mitspielern von SPARTA Göttingen e.V. danke ich für gemeinsam errungene glorreiche Siege, hart umkäpfte Unentschieden und lehrreiche Niederlagen.

Mein ganz besonderer Dank gilt meinen Eltern, Hans-Dieter und Gerlinde Haller, die immer für mich da waren und sind und ohne die mein Studium und diese Arbeit nicht möglich gewesen wären, sowie meinen Brüdern Florian, Fabian und Kilian.

Verena und Jannis danke ich einfach dafür, dass es sie gibt. Ich liebe Euch! 



\section{Lebenslauf}

Julian Haller

In den Wiesen 7

37124 Mengershausen

Geburtsdatum: 13. Februar 1978

Geburtsort: Göttingen

Staatsangehörigkeit: deutsch

1984 - 1988 Grundschule „Grebenbergschule“ in 37176 Angerstein

1988 - $1990 \quad$ Orientierungsstufe „Novalis-Schule“ in 37176 Nörten-Hardenberg

1990 - 1997 Gymnasium „Otto-Hahn-Gymnasium“ in 37081 Göttingen

07/1997 Abitur am Otto-Hahn-Gymnasium mit der Note 2,0

09/1997 - 10/1998 Zivildienst beim Arbeiter-Samariter-Bund in 37081 Göttingen

10/1998 Beginn des Studiums der Physik (Diplom) an der Georg-August-Universität Göttingen

10/2000 Vordiplom in Physik

11/2003 - 11/2004 Anfertigung der Diplomarbeit mit dem Titel „Untersuchungen über die Wechselwirkungen zwischen der Mizellkinetik und der Entmischungsdynamik an wässrigen Lösungen des nichtionischen Tensids $\mathrm{C}_{7} \mathrm{E}_{3}$ “ am Dritten Physikalischen Institut der Georg-August-Universität Göttingen

Diplom in Physik mit der Gesamtnote ,Gut'

seit $12 / 2004$

Wissenschaftlicher Mitarbeiter am Dritten Physikalischen Institut der Georg-AugustUniversität Göttingen und Anfertigung der Dissertation mit dem Titel „Ultraschallabsorptionsspektroskopie zur Untersuchung schneller molekularer Prozesse in Alkylglykosid-Lösungen“، 